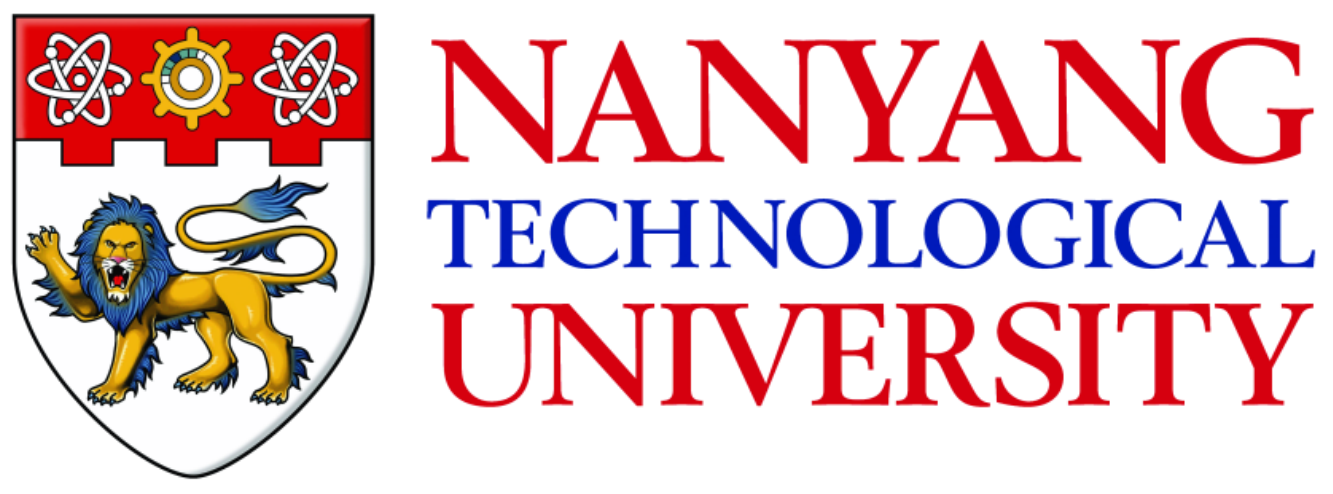

\title{
PHOTOCHROME APTAMER SWITCH ASSAY
}

\section{ZHOU YUBIN}

SCHOOL OF MATERIALS SCIENCE AND ENGINEERING 



\title{
PHOTOCHROME APTAMER SWITCH ASSAY
}

\author{
ZHOU YUBIN
}

SCHOOL OF MATERIALS SCIENCE AND ENGINEERING

A thesis submitted to the Nanyang Technological University in partial fulfilment of the requirement for the degree of Doctor of Philosophy 



\section{Statement of Originality}

I hereby certify that the work embodied in this thesis is the result of original research and has not been submitted for a higher degree to any other University or Institution. 



\section{Abstract}

Aptamers are known to undergo adaptive conformational change upon ligand binding, causing micro-environment rearrangements that may be linked with stilbene transducers. Stilbene displays fluorescence decay upon on continuous excitation, which is sensitive to micro-environment sterics. This thesis hypothesizes that fluorescence decay based biosensing can be achieved by incorporating the molecular recognition properties of aptamer with time-dependent stilbene trans-cis photodecay. Two designs were proposed and tested according to the hypothesis: 1) "competitive binding of stilbene-analyte conjugate with analyte" and 2) "stilbene-aptamer conjugate fluorescence decay correlates to analyte binding". Malachite green aptamer (MGA) and its ligand malachite green (MG) were chosen as a model study to test the hypothesis. Prior to hypothesis investigation, experimental conditions were established and optimized. The MGA/MG binding was found to have hydrolysis-dependent photodecay, but organic additives were found to reverse the hydrolysis and ultimately stabilize the binding. For the design 1), MG-stilbene conjugates obtained for the hypothesis testing had micromolar binding affinities with appreciable photodecay. Based on the MG-stilbene conjugates analyzed, electron donating needs to be optimized towards retaining the stilbene fluorescence decay property in MG-stilbene conjugate. In the design 2), stilbene was grafted on aptamer to fabricate the stilbene aptasensor through amine-isothiocyanate click chemistry. This conjugate maintained properties of aptamer binding and stilbene fluorescence decay, where presence of MG inhibited the fluorescence decay of MGA-SITS. The fluorescence decay of this aptasensor was also sensitive to ligand tetramethylrosamine (TMR) but presented no response to another non-binding triphenyl molecule rhodamine $B(\mathrm{RhB})$, displaying good selectivity. The sensitivity of the MGA-SITS aptasensor was low for MG, when compared to TMR. Future work aims to improve the performance of the fluorescence decay based aptasensor. 
Abstract 


\section{Acknowledgements}

Finally, here is a space allowing writing without rationality and logic. Yes, this page is all about emotional appreciations, which seems common but really essential. Let's go.

Definitely, I would like to present my deepest appreciation to my supervisor, Professor Terry W. J. Steele, for his excellent guidance, help, support and suggestions. His critical thinking always lights up my ideas on research work, which will play an important role throughout my research life. Without his help and support, nothing is still noting---this dissertation and the published papers would have not been possible.

I am very thankful for my beloved family for their always love and support on my study and research, even though they do not really understand what I am doing. I would really appreciate all the encouragement from my family.

I wish to express my gratitude towards my thesis committee members Professor $\mathrm{Hu}$ Xiao and Professor Ali Gilles Tchenguise Miserez for their time and valuable suggestions on my project, presentation and the thesis.

I would also like to thank my project partner Ms. Wu Yuanyuan. Her contribution greatly helps improve the project and the thesis. Thank Dr. Vladislav Papper for his professional help on photochemistry and stilbene characterization. Particularly, thank Dr. Oleksandr Pokholenko for his help in my research and for the materials he provides. Specially, thank Dr. Ping Jianfeng for his kindly help, mentoring and suggestions on my experimental work on biosensors.

Thank Professor Robert S. Marks for his suggestions and supporting on my topic.

Thank Professor Souhir Boujday for her kindly guidance on teaching me new knowledge on interface.

I am also very thankful to all the members of our group, both the current and the former group members. It is my pleasure to be in the same group with Raghavendra (Dr.), Alok (Dr.), Sing Shy (Dr.), Jianlin (Dr.), Hong (Dr.), Kumar (Dr.), Richard (Dr.), Ivan (Dr.), Lu (Dr.), Himansu (Dr.), Vishal (Dr.), Su Yin, Divya, Feng, Ting, Yuanyuan, Raphael, Ankur (Shah), Shardul, Ankur (Ruhela), Harshini, Tobias, Teilo and Eda. 
I would like to give sincere appreciation to all my teachers on my course work. I also appreciate the help from all the MSE technical and administrative staff on my research.

Thank MSE and NTU for providing me the research scholarship and the chance to study in such an amazing place. Thank MOE Tier 1 and CREATE programme for funding the project. Thank MSE laboratories, especially the B1 Research Focus Laboratory, for providing the modern experimental equipment for my project.

I would also like to thank my friends for their support in my research, and my basketballmate for helping me release from the hard lab work.

Finally, thanks to my youth. 


\section{Table of Contents}

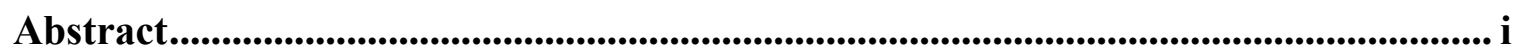

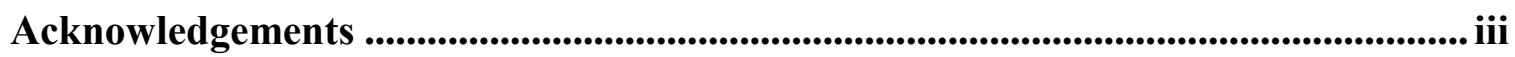

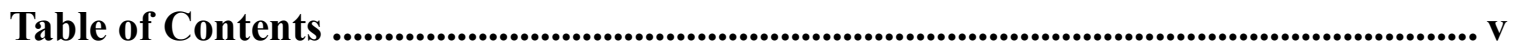

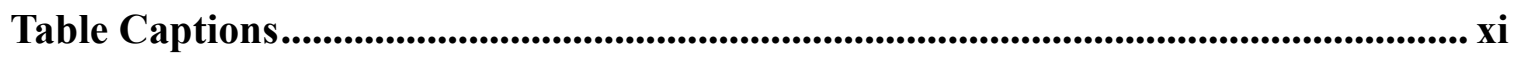

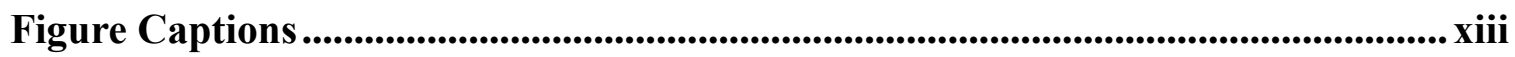

Abbreviations .......................................................................................................................... XXv

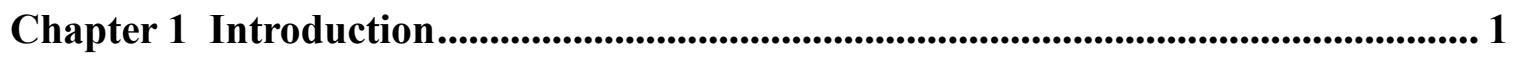

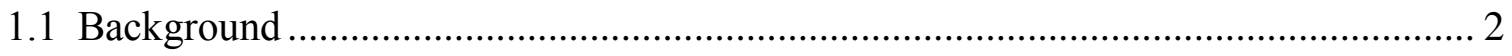

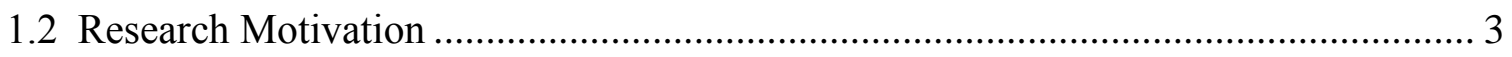

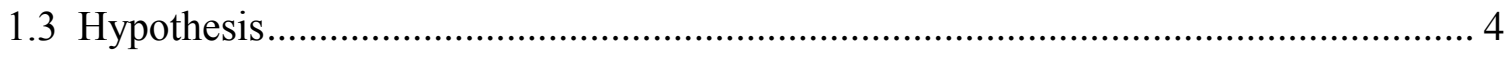

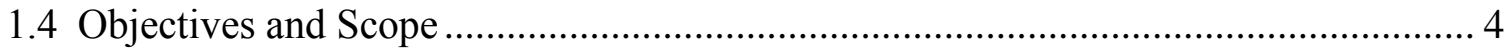

1.4.1 Competitive Stilbene-Analyte Conjugate with Analyte (Figure 1.3) ................ 4

1.4.2 Stilbene-Aptamer Conjugate Fluorescence Decay Correlates to Analyte

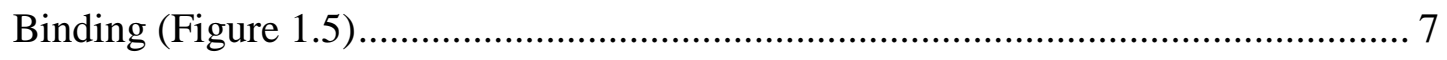

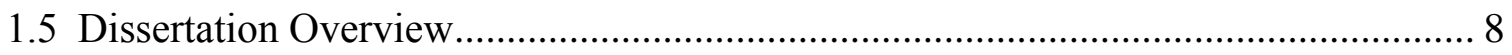

1.6 Findings and Outcomes/Originality .............................................................. 9

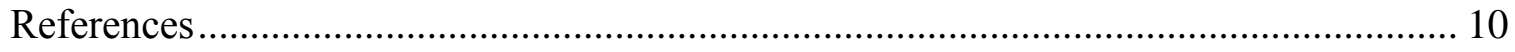

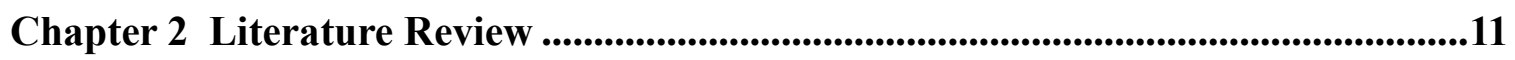

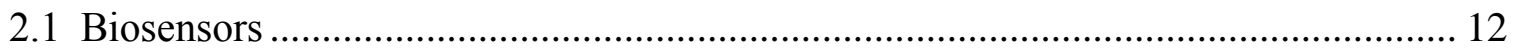


2.1.1 Definition of Biosensors ...................................................................... 12

2.1.2 Two Pivotal Elements of Biosensors ........................................................ 12

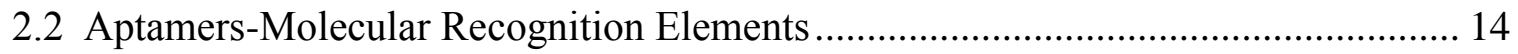

2.2.1 Definition and Present Situation of Aptamers .......................................... 14

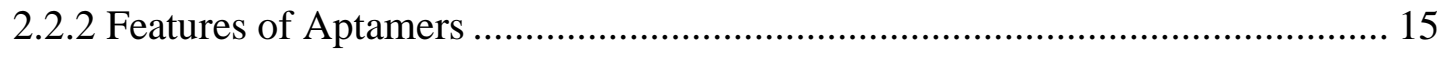

2.2.3 Methods for Measurement of Aptamer Binding Affinity ............................. 17

2.2.4 Aptamers in Biosensing Application ......................................................... 18

2.2.5 Aptamers Applied in Fluorescent Biosensors ............................................. 19

2.2.6 Malachite Green Aptamer (MGA)-A Model Study ....................................... 20

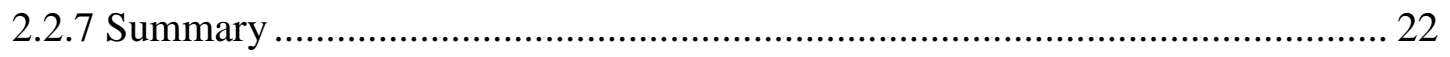

2.3 Stilbene Compounds-Reporters .................................................................. 22

2.3.1 Current Reporters and Definition of Stilbene ........................................... 22

2.3.2 Fluorescence Properties of Stilbene .......................................................... 22

2.3.3 Variation in Photoisomerization of Stilbene Compounds.............................. 23

2.3.4 Regeneration of Decayed Stilbene Compounds.......................................... 25

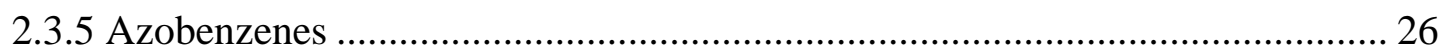

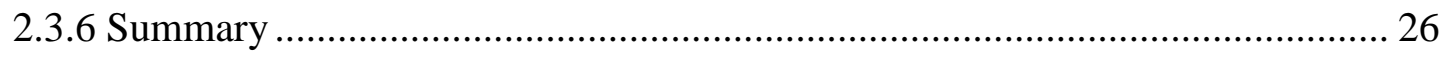

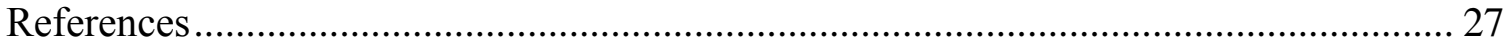

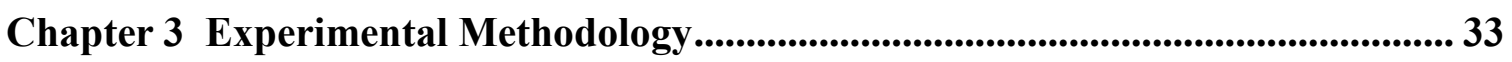

3.1 Rationale for selection ................................................................................ 34

3.2 Selection of Model Aptamer - Malachite Green Aptamer (MGA) and Malachite

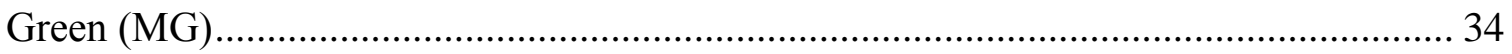

3.3 Determination of MGA Binding Affinity by High-Throughput Microplate Assay ... 36 3.3.1 High-Throughput Binding by Fluorescence Enhancement of MG .................. 36

3.3.2 Fitting Equation for $K_{\mathrm{d}}$ Determination in Direct Binding ........................... 40 
3.3.3 Determination of $K_{\mathrm{d}}$ in Competitive Binding ……….................................. 43

3.4 Fluorescence Emission and Fluorescence Decay Measurements of Stilbene

Compounds by Spectrofluorophotometer ..................................................................... 44

3.5 Preparation of MGA-SITS Conjugate (5) (Figure 3.1) ………………………....... 47

3.5.1 Synthesis and Purification of MGA-SITS Conjugate ........................................ 47

3.5.2 Characterization of MGA-SITS Conjugate...................................................... 49

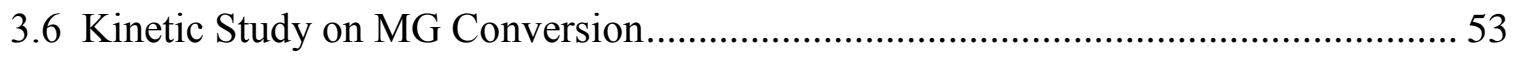

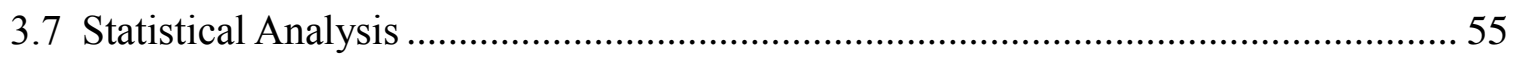

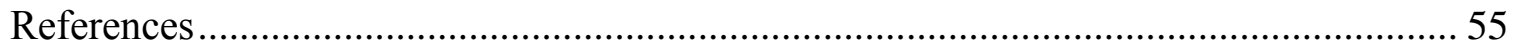

Chapter 4 Conditions for Malachite Green Aptamer Binding ...................................... 59

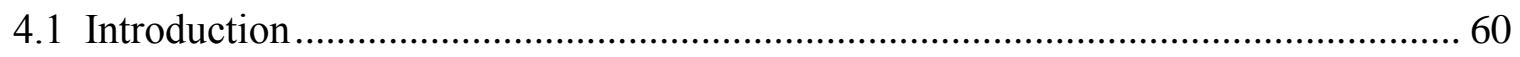

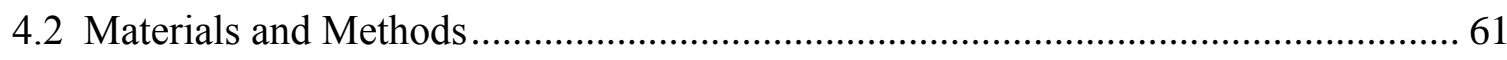

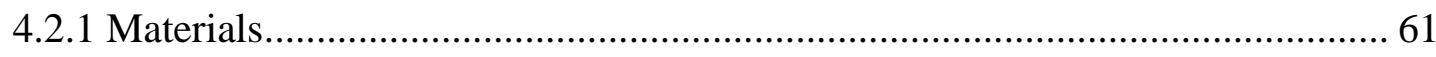

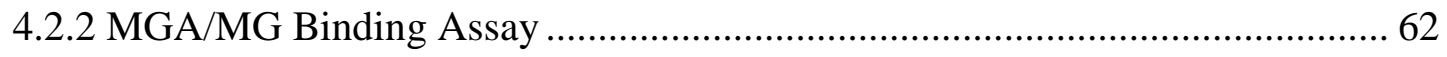

4.2.3 High Performance Liquid Chromatography (HPLC)-Diode Array Detector

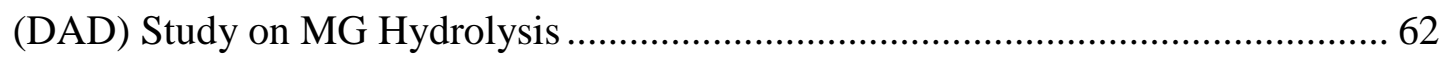

4.2.4 UV-Vis Spectral Study on Kinetic Conversion between MG and MGOH....... 63

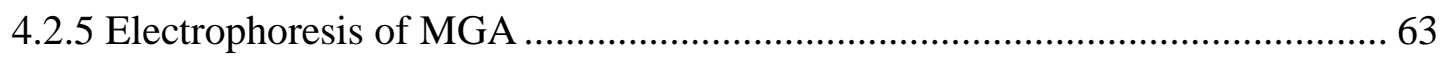

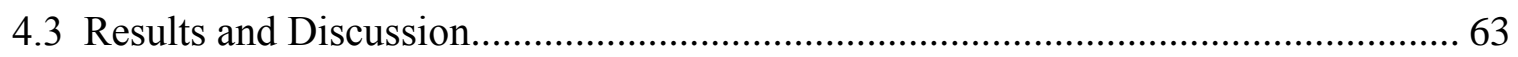

4.3.1 Effect of $\mathrm{pH}$ on Aptamer Binding Affinity ……………………………….......... 64

4.3.2 Fluorescence Intensity and Apparent Affinity of MGA/MG Complex Diminish

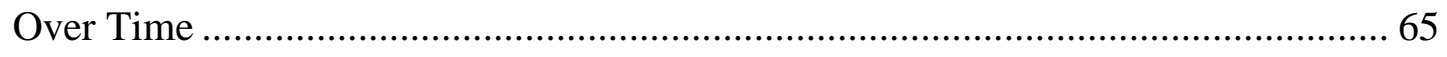

4.3.3 Addition of MG Reverses Decreased Fluorescence and MGA Keeps Stable

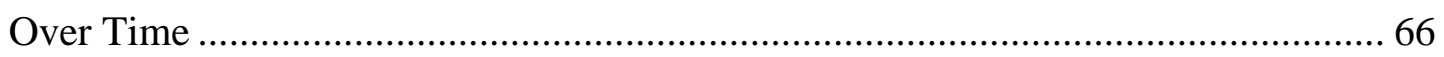

4.3.4 Loss of Fluorescence Is Attributed to Hydrolysis of MG to MGOH................. 68 
4.3.5 Organic Additives Retain Fluorescence Intensity and Binding Affinity of MG

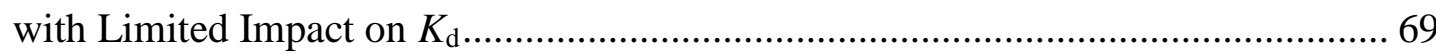

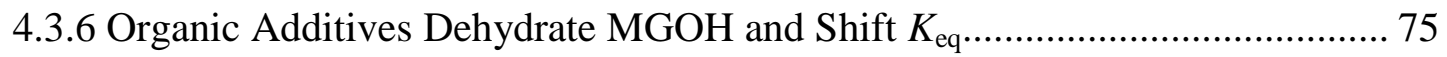

4.3.7 Organic Additives Reverse Decreased Fluorescence through MG Dehydration 77

4.3.8 Synergistic Stabilization of Ligand by Aptamer and Organic Additives.......... 79

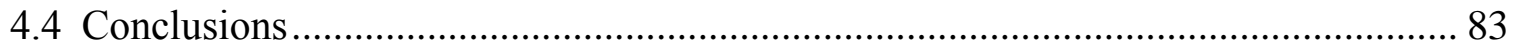

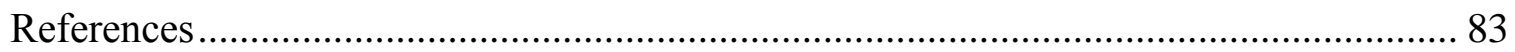

\section{Chapter 5 Binding and Fluorescence Properties of Malachite Green-Stilbene}

Conjugates .............................................................................................................................................. 85

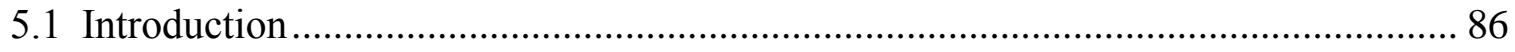

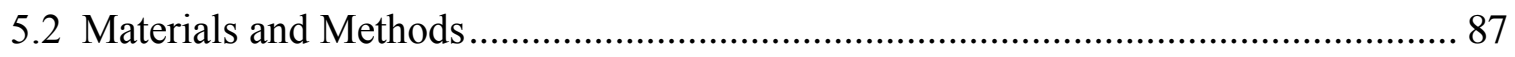

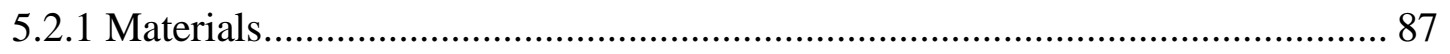

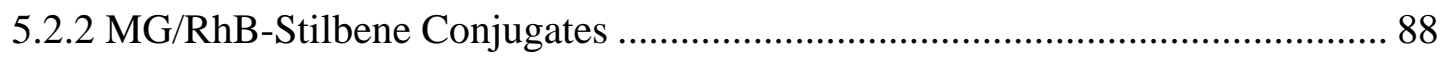

5.2.3 Binding Tests of MGA to MG, RhB and MG/RhB-Stilbene Conjugates......... 88

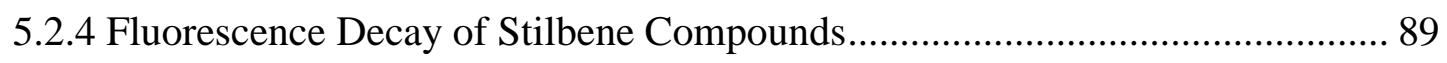

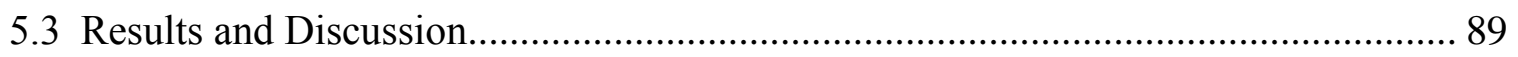

5.3.1 Binding of MGA with MG (1) and Fluorescence Decay of $\left(\mathrm{CH}_{3}\right)_{2}-\mathrm{N}$-stilbene-

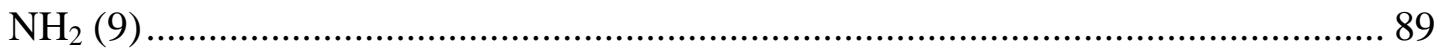

5.3.2 MGA Binding and Fluorescence Decay of RhB-stilbene-N- $\left(\mathrm{CH}_{3}\right)_{2}(11) \ldots \ldots . . .91$

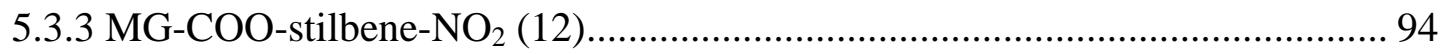

5.3.4 MG-CH

5.3.5 MG-CH

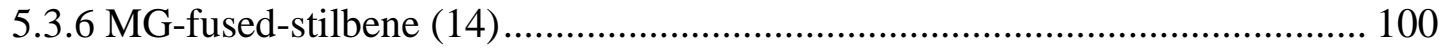

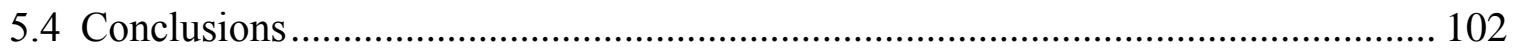


References 104

Chapter 6 Malachite Green Aptamer-Stilbene Conjugate...................................... 107

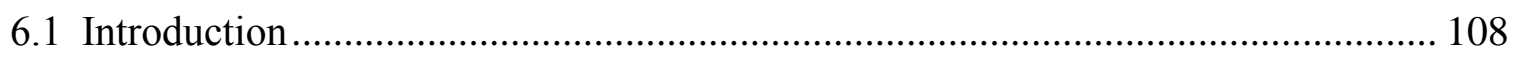

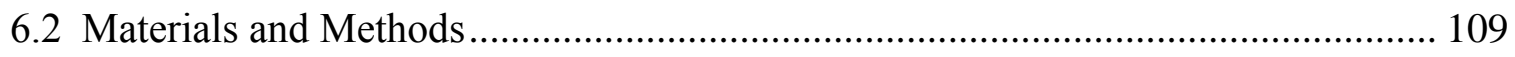

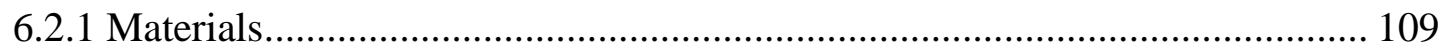

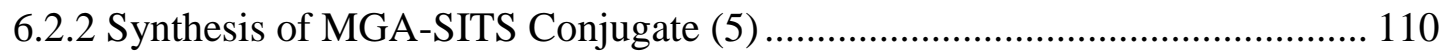

6.2.3 Characterization of MGA-SITS by High Performance Liquid Chromatography

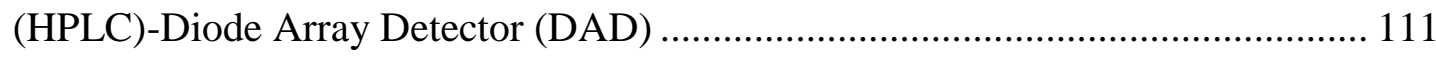

6.2.4 Matrix-Assisted Laser Desorption/Ionization Time-of-Flight (MALDI-TOF)

Mass Spectrum Characterization of MGA-SITS .............................................. 112

6.2.5 $K_{\mathrm{d}}$ Determination of MGA-SITS in Binding with MG.............................. 112

6.2.6 Measurement of MGA-SITS Fluorescence Decay and Its Aptasensing......... 112

6.2.7 Measurement of SITS Fluorescence Decay in the Presence of MG and

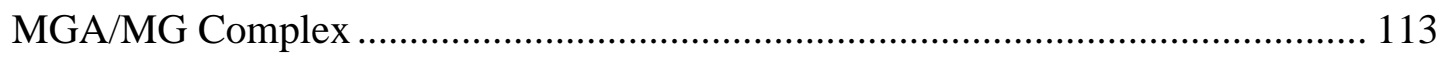

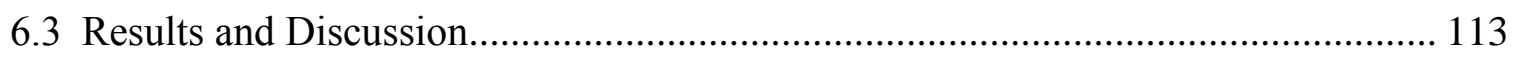

6.3.1 Successful Synthesis of MGA-SITS Conjugate with Complete Removal of Free

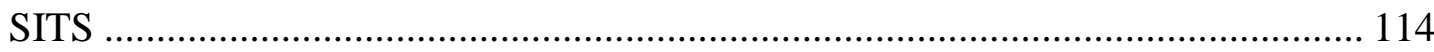

6.3.2 Optimizations on Bioconjugation Reaction ......................................... 117

6.3.3 MGA-SITS Keeps Properties of Aptamer Binding and Stilbene Fluorescence

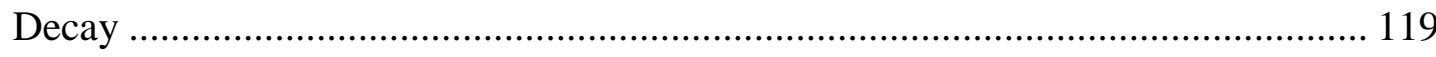

6.3.4 Fluorescence Decay of MGA-SITS Is Retarded upon MG Binding ............. 121

6.3.5 Delayed Fluorescence Decay Isolated To MGA-SITS ............................... 122

6.3.6 The MGA-SITS Conjugate Shows Selectivity of Ligands .......................... 124

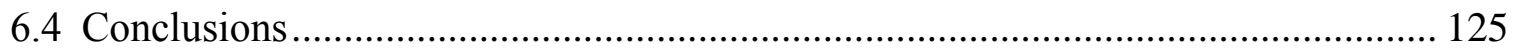

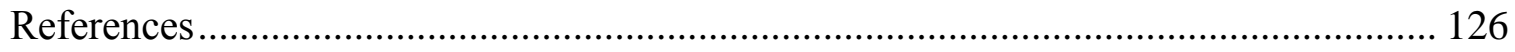


Chapter 7 Summary, Implications and Future Suggestions ................................... 129

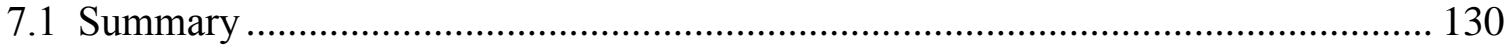

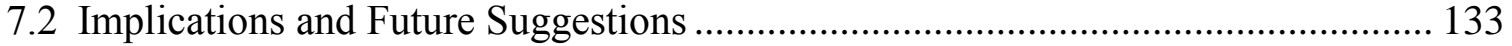

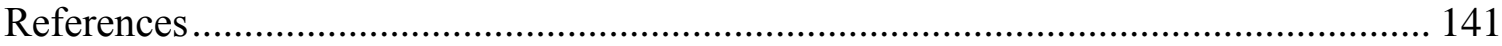

List of Publications..................................................................... 142 


\section{Table Captions}

Table 2.1 Summary of advantages of aptamers over antibodies.

Table 4.1 Decay rate constant $\left(k_{\mathrm{MG}_{\rightarrow} \mathrm{MGOH}}\right)$ of fluorescence intensity at $\mathrm{MGA}: \mathrm{MG}=$ 40:1 (maximum ratio) with various organic additive percentages.

Table 4.2 $\mathrm{MG}$ hydrolysis rate constant $\left(k_{\mathrm{MG}_{\rightarrow} \mathrm{MGOH}}\right)$ with $10 \%$ of organic additives in the presence and absence of MGA (MGA:MG = 40:1), and the theoretical calculated properties of water and additives.

Table 5.1 Summary of the binding and fluorescence results of conjugates and controls.

Table 6.1 MG binding $K_{\mathrm{d}}$ of modified MGA. 


\section{Figure Captions}

Figure 1.1 Schematic illustration of the fluorescence decay of trans-stilbene upon irradiation. (A) Stilbene exists in trans form and cis form. (B) The transstilbene exhibits fluorescence when excited, followed by a conversion to the non-fluorescent $c i s$-stilbene, where a steady-state equilibrium is reached.

Figure 1.2 Overview of the aptasensor project with project team members of $\mathrm{PhD}$ candidate $\mathrm{Wu}$ Yuanyuan and Dr. Oleksandr Pokholenko.

Figure 1.3 Schematic illustration of fluorescence decay of stilbene in competitive binding.

Figure 1.4 Fluorescence decay correlates to analyte concentrations.

Figure 1.5 Schematic illustration of the hypothesized modes of stilbene fluorescence decay upon aptameric allostery. (A) When a stilbene is grafted on aptamer, its fluorescence decay behavior is kept, and the decay is inhibited due to the adaptive aptamer binding in presence of analyte. (B) When a stilbene is grafted on aptamer, its fluorescence decay behavior is inhibited, and the decay is recovered due to the adaptive aptamer binding in presence of analyte. (C) There is no variation in the fluorescence decay of aptamerconjugated stilbene in the presence of analyte.

Figure 2.1 Schematic illustration of working principle of biosensors.

Figure 2.2 Illustration of applications of aptamers.

Figure 2.3 Schematic illustration of conformational change of aptamer upon binding.

Figure 2.4 A label-free colorimetric aptasensor based on the color change of gold 
nanoparticles (AuNPs) upon aggregation for biosensing.

Figure 2.5 Scheme of a typical graphene oxide-based fluorescent aptameric biosensor using fluorescence resonance energy transfer (FRET).

Figure 2.6 Structure of malachite green aptamer (MGA) with MG bound. 3D structure of MGA is presented by Discovery studio 3.5 Visualizer, Accelrys. Nucleic Acid Sequence: 38 mer GGAUCCCGACUGGCGAGAGCCAGGUAACGAAUGGAUCC. 3D structure of MGA (1Q8N) is presented by Discovery studio 3.5 Visualizer, Accelrys. Preferred positions for modification are marked according to the 3D conformation and the structure activity relationship.

Figure 2.7 Trans-isomer and cis-isomer of stilbene and schematic illustration of the fluorescence decay property of trans-stilbene upon UV irradiation.

Figure 2.8 Inhibition of stilbene photoisomerization by being placed in an octa acid capsule. Conformations were evidenced by ${ }^{1} \mathrm{H}$ NMR.

Figure 2.9 Schematic illustration of fluorescence change of stilbene upon photoisomerization. Trans-cis: excitation $350 \mathrm{~nm}$, emission $438 \mathrm{~nm}$, and cistrans: excitation $300 \mathrm{~nm}$, emission $438 \mathrm{~nm}$. Graph is redrawn from reference.

Figure 2.10 Chemical structures of stilbene-like compounds. (A) Trans-isomer and cisisomer of azobenzene. (B) $N$-benzylideneaniline.

Figure 3.1 Structure of malachite green aptamer (MGA), malachite green (MG) (1), MG- $\mathrm{CH}_{2}$-NH-stilbene-OMe (2), 4,4'-Diisothiocyanatostilbene-2,2'disulfonic acid disodium salt (DIDS) (3), 4-Acetamido-4'-isothiocyanato2,2'-stilbenedisulfonic acid disodium salt (SITS) (4), MGA-SITS (5) and 
malachite green carbinol base $(\mathrm{MGOH})(6) .3 \mathrm{D}$ structure of MGA (1Q8N) is presented by Discovery studio 3.5 Visualizer, Accelrys.

Figure 3.2 Jablonski diagram illustration of fluorescence emission.

Figure 3.3 Fluorescence enhancement of $\mathrm{MG}$ upon binding to MGA at excitation wavelength of $620 \mathrm{~nm}$.

Figure 3.4 An illustration of $K_{\mathrm{d}}$ determination by fitting equation EQ (1) based on the fluorescence enhancement of $\mathrm{MG}(150 \mathrm{nM})$ at ex/em $=620 \mathrm{~nm} / 656 \mathrm{~nm}$ upon binding to MGA.

Figure 3.5 An illustration of $K_{\mathrm{d}}$ determination by fitting equation EQ (13) based on the fluorescence enhancement of $\mathrm{MG}(150 \mathrm{nM})$ at ex/em $=620 \mathrm{~nm} / 656 \mathrm{~nm}$ upon binding to MGA.

Figure 3.6 An illustration of $K_{\mathrm{d}}$ determination by competitive binding of a MG-stilbene conjugate (2) to MGA/MG complex, ex/em = $620 \mathrm{~nm} / 656 \mathrm{~nm}$.

Figure 3.7 Fluorescence emission spectrum of stilbene compound DIDS (3) with $\lambda_{\mathrm{ex}}=$ $340 \mathrm{~nm}$.

Figure 3.8 Fluorescence decay spectrum at wavelength of emission maximum $\left(\lambda_{\max }\right)$ and the corresponding apparent fluorescence decay rate constant $k_{\text {app }}$ (calculated by EQ (15)) of stilbene compound DIDS (ex/em = $340 \mathrm{~nm} / 414$ $\mathrm{nm})$.

Figure 3.9 Synthesis of MGA-SITS conjugate (5).

Figure 3.10 Schematic simplified working flow/principle of HPLC and DAD. 
Figure 3.11 $\mathrm{C}_{18}$ reversed-phase HPLC-DAD chromatography of $\mathrm{MGA}-\mathrm{NH}_{2}$.

Figure 3.12 Schematic principle of MALDI-TOF mass spectrometry.

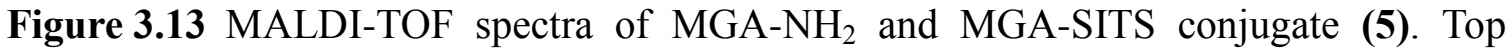
(blue): MGA-NH 2 before conjugation. Bottom (red): SITS grafted MGA$\mathrm{NH}_{2}$ (MGA-SITS conjugate (5)). The $\mathrm{m} / \mathrm{z}$ increases for 457 units after the conjugation, indicating the molecular weight of the aptamer molecule increases 457 Da upon attachment of the SITS fragment. The MALDI-TOF results indicate the SITS is successfully conjugated on MGA.

Figure 3.14 Schematic working principle of UV-Vis spectrophotometer.

Figure 3.15 An example of kinetic study on MG conversion by UV-Vis spectrophotometer.

Figure 4.1 (A) Structure of malachite green aptamer (MGA) (6), malachite green (MG) (1) and malachite green carbinol base $(\mathrm{MGOH})$. (B) Binding of MGA to MG, with fluorescence enhancement obtained.

Figure 4.2 MGA/MG binding curves at ex/em $=620 \mathrm{~nm} / 656 \mathrm{~nm}$ (A) and corresponding apparent $K_{\mathrm{d}}$ values (B) in various $\mathrm{pH}$.

Figure 4.3 (A) Binding $K_{\mathrm{d}}$ of $\mathrm{MGA} / \mathrm{MG}$ in binding buffer from 0 to $24 \mathrm{~h}$. (B) Normalized fluorescence decay at MGA:MG of 40:1 (the highest ratio).

Figure 4.4 (A) Fluorescence intensity of MGA/MG complex after $24 \mathrm{~h}$ binding and with the subsequent addition of original amount of MG (blue square) and MGA (black circle). (B) Agarose gel electrophoresis of MGA over time in binding buffer. 
Figure 4.5 HPLC of MG 0h, MG $24 \mathrm{~h}$ and MGOH. (A) Detection $\lambda=620 \mathrm{~nm}$. (B) Detection $\lambda=265 \mathrm{~nm}$.

Figure 4.6 Apparent $K_{d}$ values with organic additives at time 0 h. (A) With 3\% and 10\% ACN. (B) With 3\% and 10\% DMSO. (C) With 3\% and 10\% EtOH. (D) Variation of the apparent $K_{\mathrm{d}}$ values in different percentages of organic additives. ${ }^{*}=p<0.05$ : significant difference against apparent $K_{\mathrm{d}}$ without organic additive.

Figure 4.7 (A) Binding curves of MGA to MG with different percentage of ACN at $0 \mathrm{~h}$ and $24 \mathrm{~h}$. (B) Plots of calculated $K_{\mathrm{d}}$ values with different percentages of organic additives at $0 \mathrm{~h}$ and $24 \mathrm{~h}$. (C) Plots of fluorescence intensity at MGA:MG $=40: 1$ at $0 \mathrm{~h}$ and $24 \mathrm{~h}$ with different percentages of organic additives. NSD $=$ no statistical difference, $\mathrm{p}<0.05$.

Figure 4.8 Kinetic evolution between $\mathrm{MG}$ and $\mathrm{MGOH}$ (without aptamer). (A) $\mathrm{MG}$ converts to $\mathrm{MGOH}$ in binding buffer and in the presence of $10 \%$ organic additives. (B) $\mathrm{MGOH}$ converts to $\mathrm{MG}$ in binding buffer and in the presence of $10 \%$ organic additives.

Figure 4.9 Rate constant of $\mathrm{MGOH}$ to $\mathrm{MG}\left(k_{\mathrm{MGOH}_{\rightarrow} \mathrm{MG}}\right)$ in binding buffer in the presence of MGA.

Figure 4.10 Addition of DMSO into MGA/MG complex at 5 h. (A) 3\% v/v DMSO for 1 h. (B) $3 \% \mathrm{v} / \mathrm{v}$ DMSO for $12 \mathrm{~h}$. (C) $5 \% \mathrm{v} / \mathrm{v}$ DMSO for $1 \mathrm{~h}$. (D) $5 \% \mathrm{v} / \mathrm{v}$ DMSO for $12 \mathrm{~h}$.

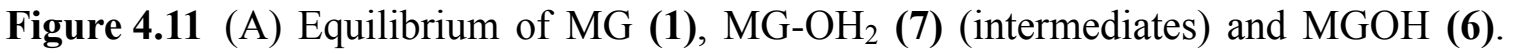
(B) Rate of $\mathrm{MG}$ elimination. At pH 6.7, $\left[\mathrm{OH}^{-}\right]$is $5.0 \times 10^{-8} \mathrm{M}$ and thus, $k_{\mathrm{OH}^{-}}$ $\left[\mathrm{OH}^{-}\right]<<k_{\mathrm{H} 2 \mathrm{O}}\left[\mathrm{H}_{2} \mathrm{O}\right]$. (C) Proposed mechanism of the conversions between 
$\mathrm{MG}, \mathrm{MG}-\mathrm{OH}_{2}$ and $\mathrm{MGOH}$ with MGA presented. Without MGA, the rate constants $k_{\mathrm{MG}_{\rightarrow} \mathrm{MGOH}}$ (hydrolysis) and $k_{\mathrm{MGOH}_{\rightarrow} \mathrm{MG}}$ (dehydration) favor $\mathrm{MGOH}$ as the major equilibrium product (A). When excess MGA is present, it retards MG hydrolysis, resulting in a decreased $k_{\mathrm{MG}_{\rightarrow} \mathrm{MGOH}}$. With the addition of organic additives, $k_{\mathrm{MGOH}_{\rightarrow} \mathrm{MG}}$ increases by few hundred percent (C), which results in a "stabilizing" effect.

Figure 5.1 Chemical structures of malachite green (MG) (1), 4-Amino-4'-(N,Ndimethylamino)stilbene $\left(\left(\mathrm{CH}_{3}\right)_{2}-\mathrm{N}\right.$-stilbene- $\left.\mathrm{NH}_{2}\right)(\mathbf{9})$, rhodamine $\mathrm{B}(\mathrm{RhB})$ (10) and $\mathrm{MG} / \mathrm{RhB}$-stilbene conjugates: RhB-stilbene-N-( $\left.\mathrm{CH}_{3}\right)_{2}$ (11), MGCOO-stilbene- $\mathrm{NO}_{2}$ (12), MG- $\mathrm{CH}_{2}-\mathrm{NH}$-stilbene-OMe (2), MG-CH $2-\mathrm{NAc}$ stilbene-OMe (13) and MG-fused stilbene (14).

Figure 5.2 MGA/MG binding curve and its calculated $K_{\mathrm{d}}$ based on the fluorescence enhancement of MG (1) at ex/em $=620 \mathrm{~nm} / 656 \mathrm{~nm}$ upon aptamer binding.

Figure 5.3 Fluorescence decay of $\left(\mathrm{CH}_{3}\right)_{2}-\mathrm{N}$-stilbene- $\mathrm{NH}_{2}(\mathbf{9})$, ex/em $=344 \mathrm{~nm} / 439 \mathrm{~nm}$.

Figure 5.4 Influence of medium viscosity on fluorescence decay rate constant $\left(k_{\mathrm{app}}\right)$ of $\left(\mathrm{CH}_{3}\right)_{2}-\mathrm{N}$-stilbene- $\mathrm{NH}_{2}$ (9). Results are from my project partner $\mathrm{Ms}$. Wu Yuanyuan.

Figure 5.5 Binding of MGA to with RhB (10). No significant change in anisotropy is observed.

Figure 5.6 Fluorescence decay of RhB-stilbene- $\mathrm{N}-\left(\mathrm{CH}_{3}\right)_{2}$ (11), ex/em $=350 \mathrm{~nm} / 445$ $\mathrm{nm}$.

Figure 5.7 Binding of MGA to with RhB-stilbene-N-( $\left(\mathrm{CH}_{3}\right)_{2}$ (11). No significant change in anisotropy is observed. 
Figure 5.8 (A) Fluorescence decay of $\mathrm{RhB}$-stilbene- $\mathrm{N}-\left(\mathrm{CH}_{3}\right)_{2}$ (11) in the presence of different concentration of MGA. (B) Normalized decay curves of A.

Figure 5.9 $k_{\text {app }}$ of $\mathrm{RhB}$-stilbene- $\mathrm{N}-\left(\mathrm{CH}_{3}\right)_{2}(\mathbf{1 1})$ in the presence of different concentration of MGA.

Figure 5.10 Competitive binding of MG-COO-stilbene- $\mathrm{NO}_{2}$ (12) to $\mathrm{MGA} / \mathrm{MG}$ complex and its calculated $K_{\mathrm{d}}$.

Figure 5.11 Fluorescence decay of MG-COO-stilbene- $\mathrm{NO}_{2}$ (12) at ex/em = $362 \mathrm{~nm} / 520$ $\mathrm{nm}$. Results are from my project partner Ms. Wu Yuanyuan.

Figure 5.12 Competitive binding of $\mathrm{MG}-\mathrm{CH}_{2}-\mathrm{NH}$-stilbene-OMe (2) to $\mathrm{MGA} / \mathrm{MG}$ complex and its calculated $K_{\mathrm{d}}$.

Figure 5.13 (A) Fluorescence decay of $\mathrm{MG}_{-} \mathrm{CH}_{2}-\mathrm{NH}$-stilbene-OMe (2) in different percentage of DMSO/binding buffer at ex/em $=324 \mathrm{~nm} / 432 \mathrm{~nm}$. (B) Normalized fluorescence decay of A. C: Apparent fluorescence decay rate constant $\left(k_{\text {app }}\right)$ of the decay curves. Results are from my project partner Ms. Wu Yuanyuan.

Figure 5.14 Binding of MG- $\mathrm{CH}_{2}-\mathrm{NAc}-$ stilbene-OMe (13) with MGA and its calculated $K_{\mathrm{d}}$ based on fluorescence enhancement.

Figure 5.15 (A) Fluorescence decay of $\mathrm{MG}-\mathrm{CH}_{2}-\mathrm{NAc}$-stilbene-OMe (13) in different percentage of DMSO at ex/em $=327 \mathrm{~nm} / 389 \mathrm{~nm}$. (B) Normalized fluorescence decay of A. C: Apparent fluorescence decay rate constant $\left(k_{\text {app }}\right)$ of the decay curves. Results are from my project partner Ms. Wu Yuanyuan.

Figure 5.16 Binding of MG-fused-stilbene (14) with MGA and its calculated $K_{\mathrm{d}}$ based on fluorescence enhancement. 
Figure 5.17 Fluorescence decay of MG-fused-stilbene (14) in different organic solvents. $\mathrm{Ex} / \mathrm{em}=318 \mathrm{~nm} / 381 \mathrm{~nm}$ for DMSO, $312 \mathrm{~nm} / 398 \mathrm{~nm}$ for $\mathrm{ACN}$ and 314 $\mathrm{nm} / 398 \mathrm{~nm}$ for EtOH, respectively. Results are from my project partner Ms. Wu Yuanyuan.

Figure 6.1 Schematic illustration of the aptasensor based on photochrome switch of stilbene and adaptive binding of aptamer. Without analyte (ligand), transstilbene grafted on aptamer undergoes free photoisomerization (fluorescent trans form to non-fluorescent cis form), resulting in rapid fluorescence decay. Upon analyte binding, conformational change of aptamer induces an inhibition of the stilbene photoisomerization, resulting in decrease in fluorescence decay kinetics, which can be utilized for biosensing.

Figure 6.2 Structures of malachite green aptamer (MGA), malachite green (MG) (1), 4Acetamido-4'-isothiocyanato-2,2'-stilbenedisulfonic acid disodium salt (SITS) (4), rhodamine B (RhB) (10), tetramethylrosamine (TMR) (15), aminocaproic acid (EACA) (16) and lysine (18). MGA 3D structure (PDB ID: 1Q8N) is presented by Discovery studio 3.5 Visualizer, Accelrys.

Figure 6.3 Synthesis of MGA-SITS conjugate (5).

Figure 6.4 HPLC-DAD characterization of (A) $\mathrm{MGA}^{-\mathrm{NH}_{2}}$, (B) SITS (4) and (C) MGA-SITS conjugate (5). Insets are the UV-Vis spectra (from DAD) at corresponding RT displaying the variation in absorbance.

Figure 6.5 MALDI-TOF spectra of $\mathrm{MGA}^{-\mathrm{NH}_{2}}$ (top blue) and MGA-SITS conjugate (5) (bottom red).

Figure 6.6 (A) Reaction of EACA (16) with SITS (4). (B) Reaction progress at 4 deg C.

(C) Reaction progress at room temperature. 
Figure 6.7 (A) Reaction of lysine (17) with SITS (4). (B) Reaction progress at 4 deg C.

(C) Reaction progress at room temperature.

Figure 6.8 (A) HPLC monitoring of absorbance increase in UV340 (grafting of SITS (4)) over time at RT 1.7 min. (B) The calculated degree of conjugation as a function of time.

Figure 6.9 (A) Fluorescence decay of MGA-SITS (5) at ex/em $=340 \mathrm{~nm} / 428 \mathrm{~nm}$. (B) Fluorescence emission of MG in the absence and presence of MGA-SITS at excitation wavelength of $620 \mathrm{~nm}$.

Figure 6.10 Binding of MGA- $\mathrm{NH}_{2}$ and MGA-SITS (5) with $\mathrm{MG}$, ex/em = $620 \mathrm{~nm} / 656$ $\mathrm{nm}$.

Figure 6.11 (A) Fluorescence decay curves of MGA-SITS (5) in the presence of increasing MG concentration. (B) Calibration plot of $k_{\text {app }}$ of MGA-SITS as a function of $\mathrm{MG}$ concentration $(0.5-50 \mu \mathrm{M})$.

Figure 6.12 (A) Fluorescence decay curves of SITS (4) in the presence of MG and MGA/MG complex. (B) $k_{\text {app }}$ of SITS upon the presence of MG and MGA/MG complex. ${ }^{*}: p<0.05$ : significant difference against SITS control (without MG and MGA) $(\mathrm{n}=3)$.

Figure 6.13 (A) Fluorescence decay curves of MGA-SITS (5) in the presence of $20 \mu \mathrm{M}$ MG, TMR (15) and RhB (10). (B) $k_{\text {app }}$ of MGA-SITS upon the addition of the analytes. $*$ : $p<0.05$ : significant difference against MGA-SITS $(\mathrm{n}=3)$.

Figure 6.14 (A) Fluorescence decay curves of MGA-SITS (5) in the presence of increasing concentration of TMR (15). (B) Calibration plot of MGA-SITS $k_{\text {app }}$ as a function of TMR concentration $(0.2-20 \mu \mathrm{M})$. 
Figure 7.1 Recycling of computational modelling and synthesis on MG-stilbene conjugates for optimal binding affinity and fluorescence decay.

Figure 7.2 Competitive binding of MG with prebound MGA-SITS/TMR complex for MG sensing.

Figure 7.3 Conjugation of MeO-stilbene-maleimide (20) with 6-Mercapoto-1-hexanol (MCH) (21).

Figure 7.4 MGA-SH/MG binding curve and its calculated $K_{\mathrm{d}}$, ex/em $=620 \mathrm{~nm} / 656 \mathrm{~nm}$.

Figure 7.5 Schematic illustration of regeneration of aptamer (A), stilbene (B) and the regenerated aptamer-stilbene conjugate sensor (C). 
Figure Captions

xxiii 



\section{Abbreviations}

\begin{tabular}{|c|c|}
\hline $\mathrm{ACN}$ & Acetonitrile \\
\hline DAD & Diode array Detector \\
\hline DIDS & 4,4'-diisothiocyanatostilbene-2,2'-disulfonic acid Disodium Salt \\
\hline DMSO & Dimethyl Sulfoxide \\
\hline ELISA & Enzyme-Linked Immunosorbent Assay \\
\hline $\mathrm{EtOH}$ & Ethanol \\
\hline FI & Fluorescence Intensity \\
\hline FP & Fluorescence Polarization \\
\hline HPLC & High-performance Liquid Chromatography \\
\hline LMG & Leucomalachite Green \\
\hline LOD & Limit of Detection \\
\hline MALDI-TOF & Matrix-Assisted Laser Desorption/Ionization Time-of-Flight \\
\hline MG & Malachite Green \\
\hline MGA & Malachite Green Aptamer \\
\hline MGA-NH ${ }_{2}$ & Amine Modified Malachite Green Aptamer \\
\hline MGOH & Malachite Green Carbinol Base \\
\hline MPSA & Molecular Polar Surface area \\
\hline NMR & Nuclear Magnetic Resonance \\
\hline SEC & Size Exclusion Chromatography \\
\hline RT & Retention Time \\
\hline SELEX & Systematic Evolution of Ligands by Exponential Enrichment \\
\hline SITS & 4-acetamido-4'-isothiocyanato-2,2'-stilbenedisulfonic acid disodium salt \\
\hline TMR & Tetramethylrosamine \\
\hline UV-Vis & Ultraviolet-visible \\
\hline XRD & X-ray Diffraction \\
\hline
\end{tabular}




\section{Chapter 1}

\section{Introduction}

This chapter describes the background of biosensors, aptamers and stilbenes (section 1.1), followed by an introduction of the research motivation, hypothesis and scopes (section 1.2-1.4). The advantages of aptamers and stilbenes are illustrated, providing the rationale for the hypothesis. Two designs according to the hypothesis are displayed with corresponding scopes (section 1.4). Finally, the overview of the dissertation (section 1.5) and the outcomes of the research (section 1.6) are also outlined. 


\subsection{Background}

Aptamers are a kind of synthetic oligonucleotides discriminated by Systematic Evolution of Ligands by EXponential enrichment (SELEX) technique and in vitro screening, which can bind to a wide range of targets (small molecules, proteins, or whole cells). As molecular recognition elements, aptamers have some advantages over antibodies in fabrication of the biosensors including in vitro screening and non-animal synthetic origins [1-3]. Especially, aptamers undergo adaptive conformational change upon ligand recognition, inducing micro-environment rearrangements. Currently, fluorescence biosensors mainly rely on fluorescence intensity or quenching of fluorescence reporters (probes or labels) for analyte quantification. However, this detection mode suffers from several drawbacks, such as the laborious and tedious washing procedures for the separation and background autofluorescence.

Stilbene compounds so far have never been used as the molecule sensing entity in the biosensing devices and bioassays, although they possess many of the desired features of ideal reporter molecules, for instance, chemical and biological stability, biocompability, synthetic availability, high photochemical sensitivity, rapid response and easy regeneration. Stilbene molecules are capable of changing their molecular configurations with fluorescence decay upon UV irradiation, which is sensitive to micro-environment sterics. There are two possible stilbene conformational isomers: trans and cis, as shown in Figure 1.1A. In case of a stilbene molecule, the sterochemcial trans-isomer is strongly fluorescent while the cis-isomer is non-fluorescent. Hence, irradiation of the fluorescent trans form of the reporter molecule at the excitation maximum produces a substantial change of the fluorescence emission that can be monitored as a time trace (kinetic decay) signal or emission gradient, illustrated in Figure 1.1B [4-6]. 

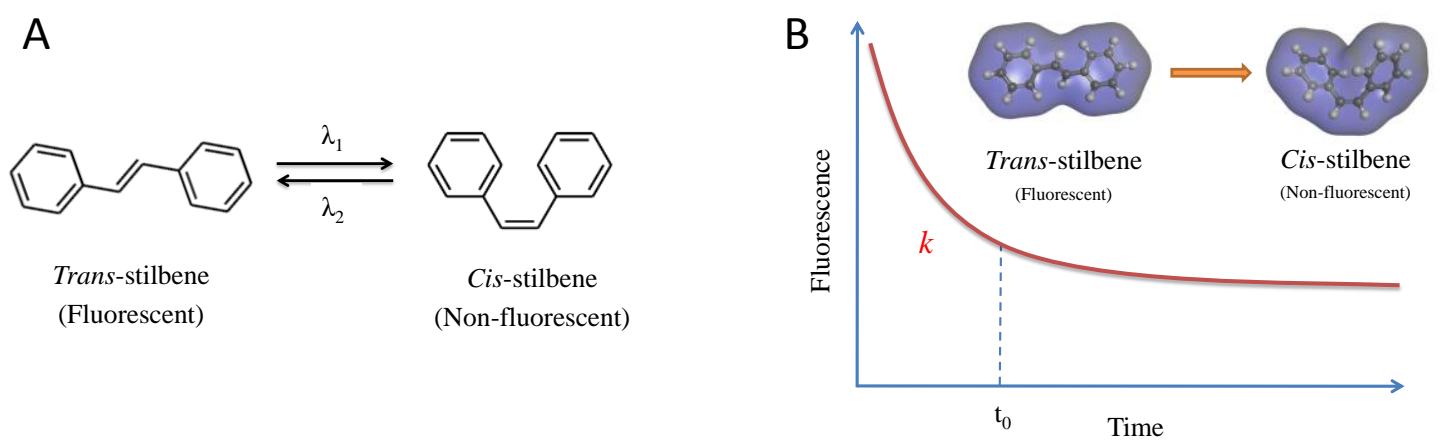

Figure 1.1 Schematic illustration of the fluorescence decay of trans-stilbene upon irradiation. (A) Stilbene exists in trans form and cis form. (B) The trans-stilbene exhibits fluorescence when excited, followed by a conversion to the non-fluorescent cis-stilbene, where a steady-state equilibrium is reached.

\subsection{Research Motivation}

The molecular recognition properties of aptamers and the fluorescence decay feature of stilbene compounds make them promising materials in the development of robust, simple, and rugged fluorescent biosensing assay. To date, research on an aptasensor using stilbene as reporter has not been found yet. Herein, we attempt to utilize aptamers and stilbene compounds to develop a fluorescence decay-based biosensor device, regardless of absolute fluorescence intensity, which can remove laborious washing procedures. Figure 1.2 displays the overlapping contribution with my project team members, $\mathrm{PhD}$ candidate $\mathrm{Wu}$ Yuanyuan and Dr. Oleksandr Pokholenko. 


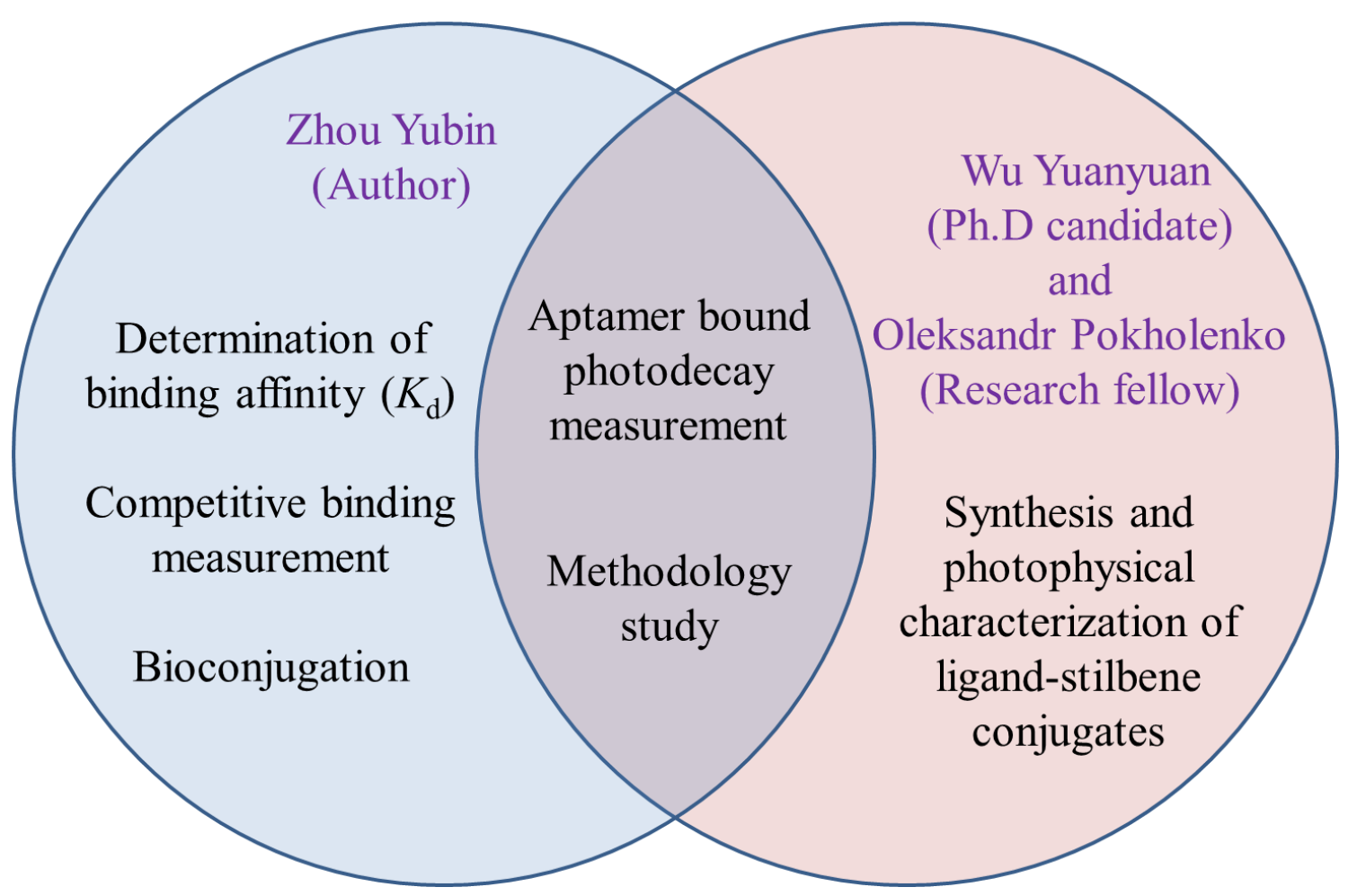

Figure 1.2 Overview of the aptasensor project with project team members of $\mathrm{PhD}$ candidate Wu Yuanyuan and Dr. Oleksandr Pokholenko.

\subsection{Hypothesis}

Changes in surrounding steric and viscosity micro-environment can be quantitated by the fluorescence decay of stilbene; aptamers are known to change their microenvironments upon binding ligands or undergo dynamic binding upon ligand docking; therefore by conjugating ligands to stilbene or stilbene directly to an aptamer, changes in aptamer binding or dynamic folding can be directly quantitated through stilbene fluorescence decay.

\subsection{Objectives and Scope}

Here are two designs and scopes for the hypothesis:

\subsubsection{Competitive Stilbene-Analyte Conjugate with Analyte (Figure 1.3)}


A stilbene-analyte conjugate has fluorescence decay property similar to stilbene. When this conjugate binds to corresponding aptamer, the whole conjugate is enclosed or immobilized by the aptamer and the fluorescence decay is retarded. When analyte is added in the aptamer/conjugate complex, it will compete and free the aptamer bound, resulting in an analyte-dependent recovery of the fluorescence decay.

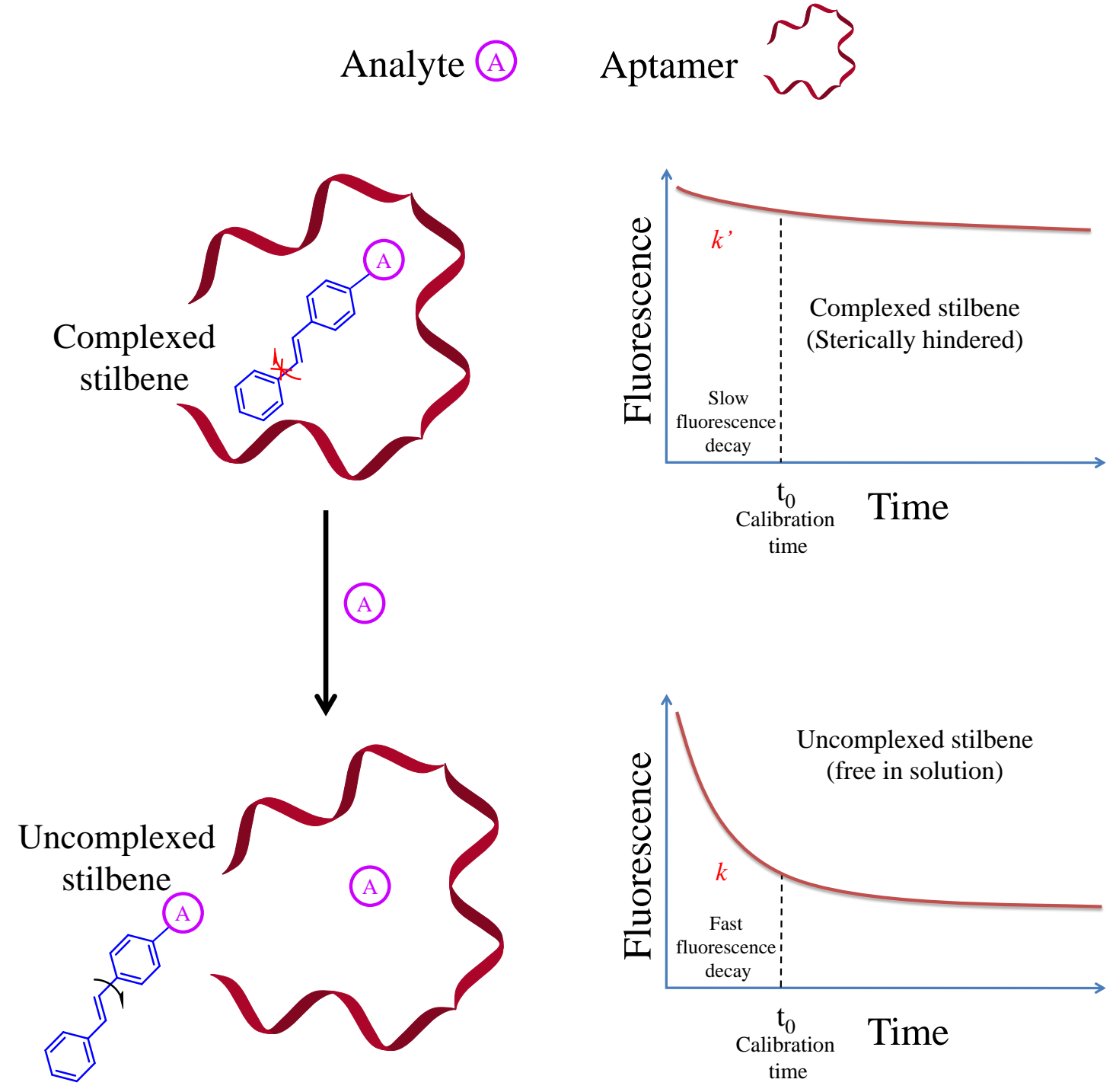

Figure 1.3 Schematic illustration of fluorescence decay of stilbene in competitive binding.

Scope for design "competitive stilbene-analyte conjugate with analyte": 
1) Determination of the binding dissociation constant $\left(K_{\mathrm{d}}\right)$ of the trans-stilbeneanalyte conjugate. The binding ability of the conjugate will be obtained by determining the $K_{\mathrm{d}}$.

2) Fluorescence decay measurement of the aptamer-bound conjugate. The fluorescence decay feature of the conjugate upon binding to aptamer will be correlated to conjugate fluorescent decay.

3) Fluorescence decay measurement of the aptamer-bound conjugate in competition against target analyte. The fluorescence decay of the "aptamer/conjugate" complex upon adding analyte will be correlated against initial fluorescent decay.

4) Calculation of the first order decay rate constant and calibration of the system. Calibration curve of first order decay rate constant vs. analyte concentration, establishing a standard curve for quantitation (Figure 1.4).
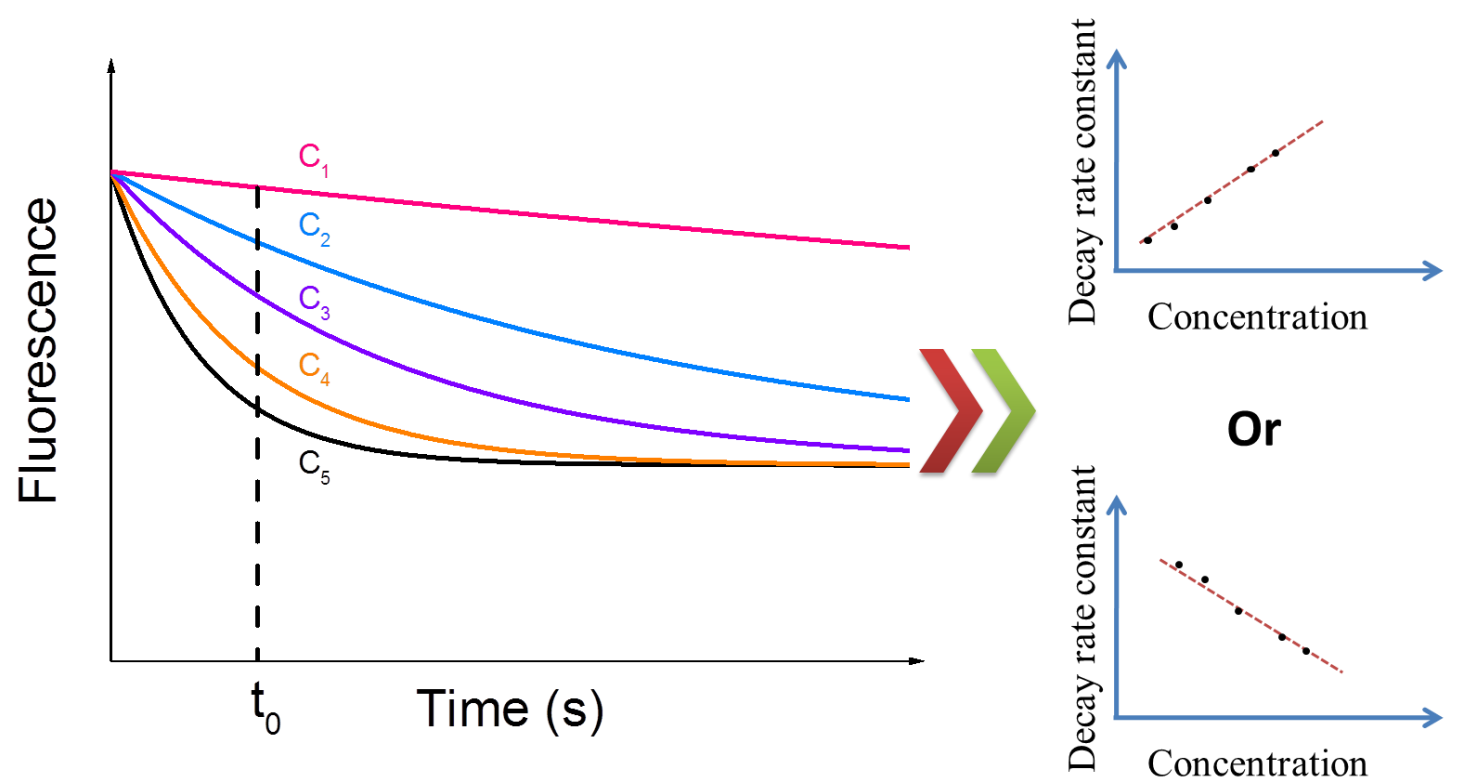

Figure 1.4 Fluorescence decay correlates to analyte concentrations. 


\subsubsection{Stilbene-Aptamer Conjugate Fluorescence Decay Correlates to Analyte Binding (Figure 1.5)}

When a stilbene is modified on aptamer, its fluorescence decay behavior will be different in the absence and presence of analyte, due to the aptamer conformational rearrangement upon analyte binding, which can affect the micro-environment surrounding stilbene.

$$
\text { Analyte (A) Aptamer } L^{2} \sigma^{1}
$$

A
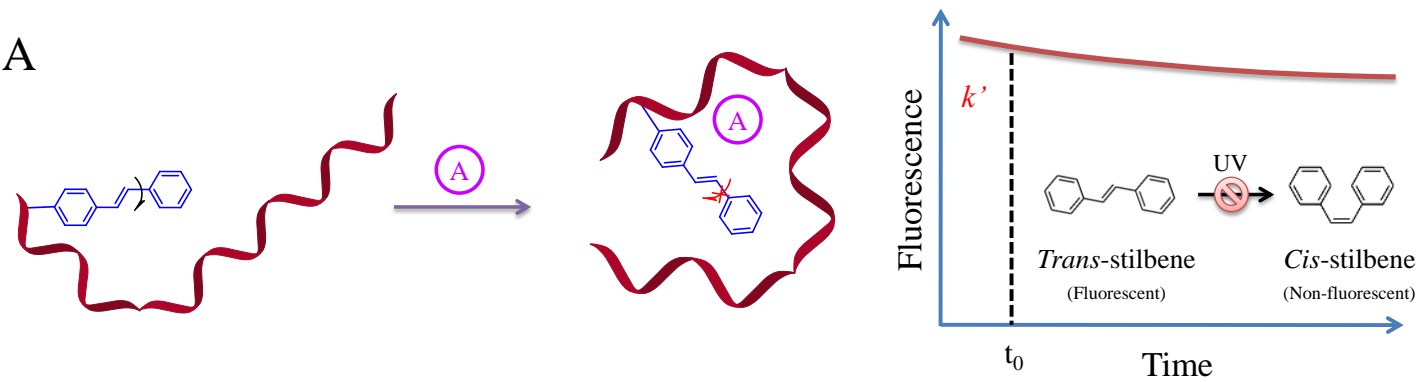

$\mathrm{B}$
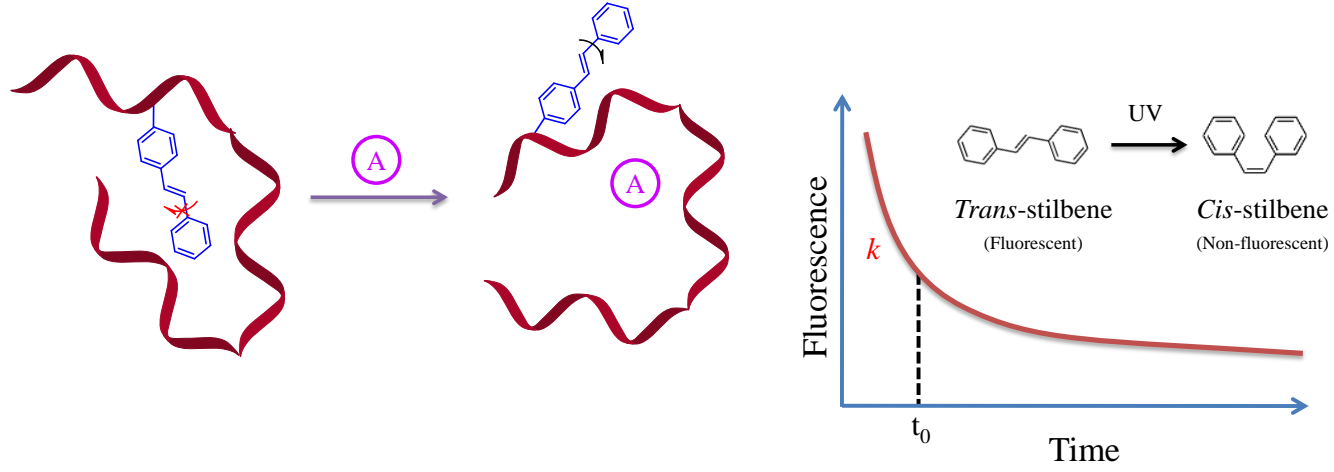

C No variation in fluorescence decay

Figure 1.5 Schematic illustration of the hypothesized modes of stilbene fluorescence decay upon aptameric allostery. (A) When a stilbene is grafted on aptamer, its fluorescence decay behavior is kept, and the decay is inhibited due to the adaptive aptamer binding in presence of analyte. (B) When a stilbene is grafted on aptamer, its fluorescence decay behavior is inhibited, and the decay is recovered due to the adaptive aptamer binding in presence of analyte. (C) There is no variation in the fluorescence decay of aptamer-conjugated stilbene in the presence of analyte.

Scope for design "stilbene-aptamer conjugate fluorescence decay correlates to analyte binding": 
1) Determination of the $K_{\mathrm{d}}$ of the trans-stilbene-aptamer conjugate to the analyte.

2) Fluorescence decay measurement of the trans-stilbene-aptamer in the presence and absence of analyte.

3) Calculation of the first order decay rate constant as a function of analyte concentration. (Figure 1.4).

If any of the designs above can be proved to have fluorescence decay change due to the addition of analyte, the hypothesis will be supported by the scope described above and challenged against the malachite green aptamer (MGA).

\subsection{Dissertation Overview}

The thesis consists of the following seven chapters:

Chapter 1 briefly describes the research background of the study, followed by an introduction of research motivation, hypothesis and scopes, and finally outlines the overview and outcomes of the thesis.

Chapter 2 reviews the historical and state-of-the-art background of biosensors, aptamers and stilbenes, with research gaps highlighted. The advantages of aptamers and stilbenes are reviewed within a biosensor perspective. A model system, comprising of malachite green aptamer, is proposed to challenge the hypothesis above.

Chapter 3 introduces the main experimental methods or techniques and their corresponding principles, including $K_{\mathrm{d}}$ determination, bioconjugation, and fluorescence decay analysis.

Chapter 4 refines the conditions for $\mathrm{MGA} / \mathrm{MG}$ binding. $\mathrm{pH}$ and organic additives 
correlations on MGA/MG binding is demonstrated. The stability of MGA, MG and bound MGA/MG is experimentally assessed and vindicated. Organic additives and their effects on MG hydrolysis and subsequent MGA binding are summarized.

Chapter 5 demonstrates MG-stilbene conjugate binding affinities and inherent fluorescence decay kinetics. The relationship between the design of MG-stilbene conjugates and their binding provides structure activity relationships in aptasensor construction.

Chapter 6 summarizes fluorescent biosensing using the MGA-stilbene aptasensor conjugate. Binding affinities of the conjugate and the aptamer before conjugation are compared, demonstrating little to no loss in affinity after conjugate construction, and conjugate maintains fluorescence decay of stilbene. The aptasensor conjugate supports our hypothesis under the limited experimental conditions described in the scope.

Chapter 7 concludes the results according to the hypothesis, and gives recommendations towards more advanced aptasensors based on stilbene transducers.

\subsection{Findings and Outcomes/Originality}

This research led to several novel outcomes:

1. Optimized binding conditions for $\mathrm{MGA} / \mathrm{MG}$ binding were found and a simple way to stabilize MGA binding was developed with common organic additives. Inhibition effect of aptamer and organic additives on ligand lability was presented.

2. MG-stilbene conjugates were synthesized and their binding affinities to MGA and fluorescence properties were measured for the first time, providing structure activity relationships required in ligand-stilbene aptameric biosensors.

3. MGA-stilbene conjugate was synthesized successfully, with a linear correlation of 
fluorescence decay kinetics and concentrations of MG and tetramethylrosamine (TMR) as model analytes. Aptasensor based on stilbene-aptamer conjugates is demonstrated for the first time.

\section{References:}

[1] K. Ikebukuro; W. Yoshida; K. Sode In Aptamers in Bioanalysis; John Wiley \& Sons, Inc.: 2008, p 129.

[2] Y.-X. Wang; Z.-Z. Ye; C.-Y. Si; Y.-B. Ying Chinese Journal of Analytical Chemistry 2012, 40, 634 .

[3] A. Chen; S. Yang Biosensors and Bioelectronics 2015, 71, 230.

[4] F. B. Mallory; C. S. Wood; J. T. Gordon; L. C. Lindquist; M. L. Savitz Journal of the American Chemical Society 1962, 84, 4361.

[5] D. H. Waldeck Chemical Reviews 1991, 91, 415.

[6] O. Chen; R. Glaser; G. I. Likhtenshtein Journal of Biochemical and Biophysical Methods 2008, 70, 1073. 


\section{Chapter 2}

\section{Literature Review}

This chapter introduces the state-of-the-art background of biosensors, aptamers and stilbenes. The basic information of biosensors and their principal elements are reviewed, with research gaps described. Advantages and unique properties of aptamers (like adaptive binding) and stilbenes (like fluorescence decay) are described and compared to other biosensor elements. Finally, a model biosensor malachite green aptamer (MGA) is introduced. 


\subsection{Biosensors}

\subsubsection{Definition of Biosensors}

Biosensors are devices that use a molecular recognition element coupled to a signal reporter (transducer) to detect an analyte of interest [1,2]. The advantages of biosensors such as high sensitivity, high specificity, portability and rapid analysis for both in situ and laboratory detection have made biosensors powerful tools for detection in medical diagnostics, environmental monitoring, security applications and food safety, among many others [3-8]. In 1962, scientist Leyland C. Clark developed an "enzyme electrode", which started the history of biosensors $[8,9]$.

\subsubsection{Two Pivotal Elements of Biosensors}

From the definition, a biosensor includes two main elements: molecular recognition element and signal reporter (transducer). An analyte interacts with the biological molecular recognition element with biological response generated, and then the recognition process is transduced to be a signal by the reporter. Finally, the signal from reporter will be usually read and recorded by corresponding equipment [2]. The working principle of biosensors is schematically shown in Figure 2.1 [2,10,11]. 


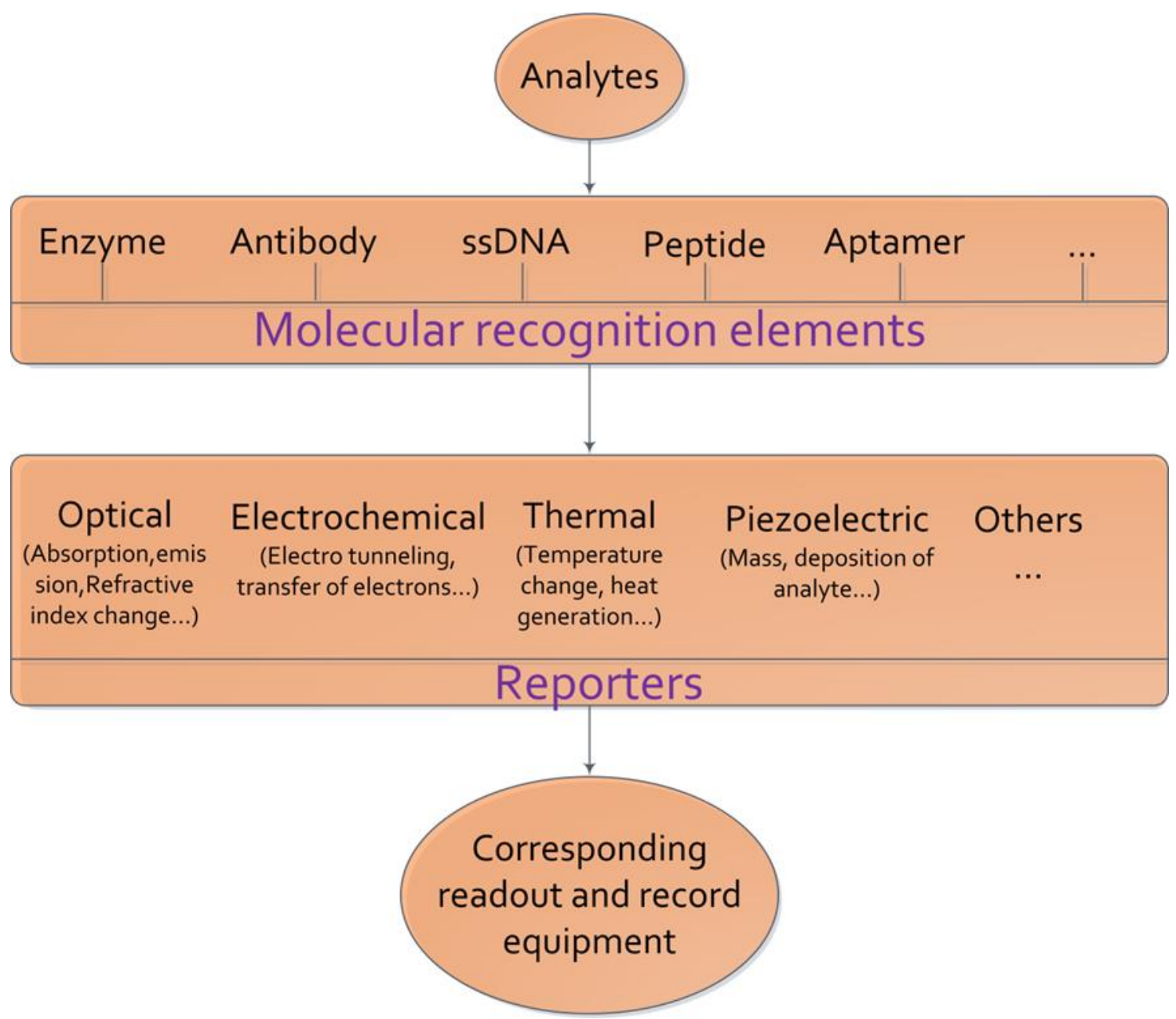

Figure 2.1 Schematic illustration of working principle of biosensors $[2,10,11]$.

Molecular recognition element is the core of a biosensor. To date, there are different kinds of molecular recognition elements that have been discovered and commonly used: antibodies, aptamers, enzymes, phages, cell-surface molecules, oligonucleotide probes, tissues, receptors, hormones, molecularly imprinted polymers and so on [11-15]. Enzymes are widely used in biosensors and their commercial biosensors for glucose measurement in blood have occupied the main part of the market. However, they suffer from lifespan and robustness $[14,16]$. Antibodies providing high affinity and specificity have been applied in enzyme-linked immunosorbent assay (ELISA) optical sensing, which is a widespread application [8,17]. However, the intrinsic shortcomings of antibodies limit their applications, which will be compared with aptamers in the following section 2.2.2. Among these molecular recognition elements, aptamers as recognition molecules have unique merits, such as chemical stability, adaptive binding 
and ease of modification, which antibodies do not possess [18].

As for reporters, electrochemical, thermal, optical, mass, piezoelectric, acoustic and mechanical reporters have been utilized in biosensing [11,14,19]. Different reporters can be selected according to the properties of molecular recognition elements and specific purposes. Optical reporters have been extensively involved in biosensors for toxins and foodborne pathogens detection [2], and optical aptameric biosensors have simple operation, remarkable sensitivity and rapid detection [20]. Fluorescence-based (optical) reporters are often used for biosensing due to their high sensitivity and simple operation [14], especially in the combination use of aptasensors [21]. However, the fluorescencebased reporters (probes or labels) are not free of shortcomings. They are now mainly based on the "Signal on/off" mode [22] and they rely on fluorescence intensity quantification or fluorescence quenching, which can be easily affected by various factors, resulting in inaccurate results. Moreover, enzyme-linked immunosorbent assay (ELISA)type fluorescent biosensors require time-consuming washing steps, and do not allow realtime detection [17,23]. Stilbene molecules are fluorescent reporters that undergo instant trans-cis conformational conversion upon irradiation. At the same time, the quantum yield diminishes because of the trans-cis conversion [24,25]. This decrease in quantum yield leads to a fluorescence decay that can be employed to correlate shifts in local micro-environments and ultimately develop a separation-free biosensor.

\subsection{Aptamers-Molecular Recognition Elements}

\subsubsection{Definition and Present Situation of Aptamers}

The term aptamer is from Latin aptus, meaning "to fit" [26]. It was first discovered by Gold's and Szostak's groups in 1990 and named by Szostak's group [27,28]. Aptamers are typically artificial oligonucleic acid molecules (single stranded DNA or RNA ligands) which can bind to a broad range of non-nucleic acid targets with high specificity and affinity, including drugs, small molecules, growth factors, peptides, enzymes, spores, toxins, proteins, whole cells and ions [29-32]. Hydrogen bonds, stacking interactions, 
electrostatic interactions and hydrophobic effect are the driving force commonly involved in the binding events, and they are related to the functional groups of targets $[33,34]$. Aptamers as biomaterials have been studied in a range of fields, such as diagnosis, biosensing, bioimaging, drug delivery, drug discovery, etc [18,35]. Applications of aptamers are shown in Figure 2.2 [35].

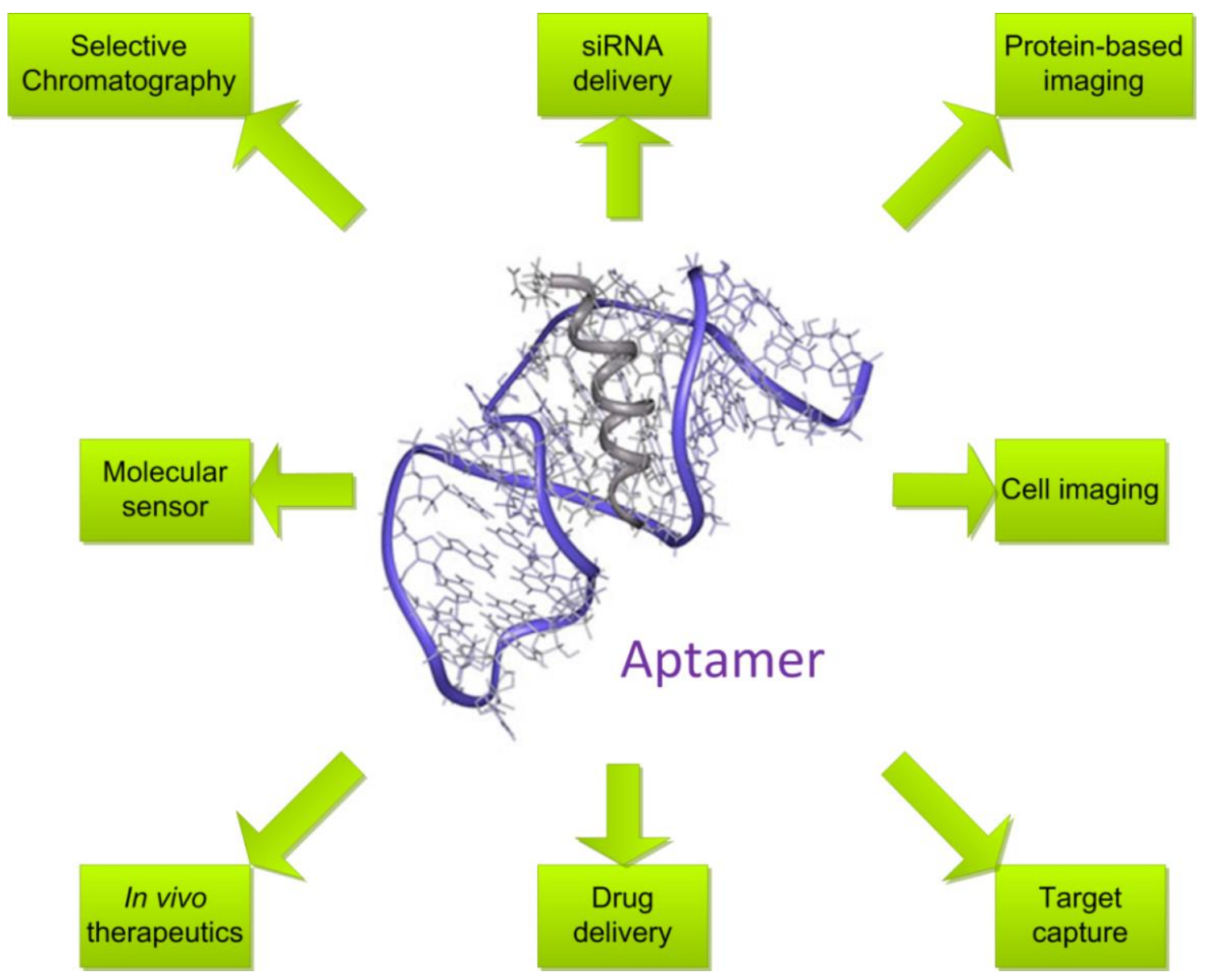

Figure 2.2 Illustration of applications of aptamers [35].

\subsubsection{Features of Aptamers}

Aptamers can be obtained from random pools of RNA or DNA in vitro via Systematic Evolution of Ligands by EXponential enrichment (SELEX) [27,28]. Aptamers are termed "chemical antibodies" because of this artificial process, which have been suggested to be good candidates towards the replacement of antibodies in biosensing [36-38]. From the SELEX process, aptamers can be selected with a wide range of targets [39,40]. 
A significant advantage of aptamers is their large-scale chemical synthesis, which makes them more cost-effective and avoids any animal origins. This reduces batch-to-batch variability and allows them to work under a range of conditions where antibodies may be hindered.

Aptamers' synthetic nature allows them to be easily modified [41], which is problematic for other types of molecular recognition elements. This allows them to be easily tagged with reporters such as fluorophores, nanoparticles and quantum dots $[18,42]$.

A unique feature of aptamers is their "adaptive binding" [43], which means aptamers will undergo a conformational change upon binding [44]. Aptamers exhibit random structures in the absence of targets while they form a defined structure upon adaptive binding [45]. Various aptameric sensors have been reported widely based on the structural switching of aptamers [46-51]. Figure 2.3 illustrates the conformational change of aptamer upon binding to ligand [52,53]. Micro-environment rearrangements can induce a difference in signal for sensing $[21,52]$. The "adaptive binding" results in a rigid and stable structure that can protect them from nuclease digestion [54]. Moreover, aptamers, like other nucleotide constructs, can recover their native structures upon thermal annealing or washing [55], allowing biosensors to be designed for multiple-used analyses with lower cost. Table 2.1 summarizes the advantages of aptamers over antibodies [56-58].

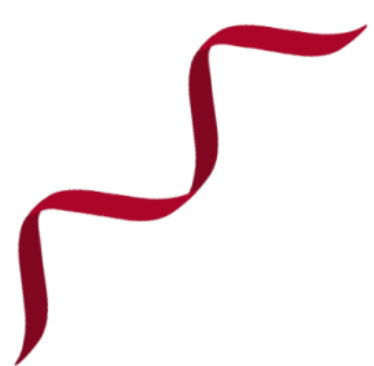

Free aptamer

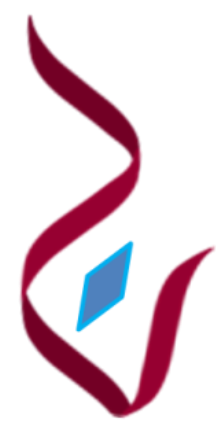

Bound aptamer

Figure 2.3 Schematic illustration of conformational change of aptamer upon binding $[52,53]$. 
Table 2.1 Summary of advantages of aptamers over antibodies [56-58].

\begin{tabular}{lll}
\hline Properties & Aptamers & Antibodies \\
\hline Design of structural change & Easy & Hard \\
Construction of conjugate & Easy & Hard \\
Affinity & Low nM-pM & Low nM-pM \\
& Wide: small molecules, drug, & \\
& organic molecules, ions, toxin, & Narrow: only immunogenic \\
Range of targets & peptides, proteins, whole & compounds \\
& cells, etc. & \\
Chemical modification & Easy and straightforward & Limited \\
Thermal denaturation & Reversible & Irreversible \\
Production & In vitro chemical process & In vivo biological system \\
Batch to batch variation & Little or no & Significant \\
Stability & High & Low \\
\hline
\end{tabular}

\subsubsection{Methods for Measurement of Aptamer Binding Affinity}

Various techniques have been developed to measure aptamer binding affinity (or equilibrium dissociation constant, $K_{\mathrm{d}}$ ): dialysis, filtration, fluorescence intensity $(\mathrm{FI})$, fluorescence polarization (FP), ultraviolet-visible (UV-Vis) absorption, surface plasmon resonance (SPR), isothermal titration calorimetry (ITC), etc [59]. Among these techniques, FI can give a simple, rapid and sensitive measurement. By incorporating the microplate reader, high-throughput FI method can be developed for $K_{\mathrm{d}}$ determination. This technique, utilizing changes in fluorescence signal, requires modification of aptamer or fluorescent signal change of ligand upon binding. FI strategy is preferred for ligands whose fluorescence can be activated by aptamer binding. FP method which distinguishes light polarization between bound and free status, is a preferred method for fluorescent molecules, without requiring absolute fluorescence intensity change in binding events. This method is suitable for $K_{\mathrm{d}}$ determination of aptamers with fluorescent ligands in relatively small molecule weight. 


\subsubsection{Aptamers in Biosensing Application}

Compared to the therapeutic field, aptamers are more attractive in the biosensing field. Aptamers have opened up exciting fields in the research of sensing interest $[34,44,60]$. An aptamer-based protein precipitation kits (AMSBIO) has been marketed capable for reuse for several times [61]. The $K_{\mathrm{d}}$ of aptamers binding to targets are commonly in the picomolar to micromolar range [21], which compares well to that of antibodies. Although there are many other commonly used recognition molecules such as antibodies, enzymes, ssDNA, peptides and receptors [14], aptamers have their advantages over other recognition molecules of biosensor: ease in synthesis and generation, a diverse range of possible target molecules, adaptive binding, easy modification, high target affinity, structural and functional robustness, and so on $[39,62]$. Aptamers have been proven to be suitable for molecular recognition of targets in biosensing [34,44]. For instance, a labelfree colorimetric aptasensor has been developed based on the color change of gold nanoparticles (AuNPs) upon aggregation (Figure 2.4) [63] and similar strategy can also been applied for other aptamers [64,65]. As a molecular recognition element in biosensing, aptamers have comparable binding specificity and affinity to antibodies, which are well known and extensively used biosensors, and thus aptamers are widely known as a substitute for antibodies [18]. Moreover, as seen in Table 2.1 aptamers possess various unique advantages and overcome the weaknesses of antibodies $[18,66]$. Particularly, detection of small molecules (like malachite green (MG)) by aptasensors is a promising direction as it is difficult to generate antibodies for small molecules, although this kind of aptasensing still remains a challenge currently [22,48,67]. Additionally, the adaptive binding of aptamers enables their sensing strategies based on conformational change, such as fluorescence resonance energy transfer (FRET) [44,68], which directly provides foundation for the hypothesis. 
Unprotected AuNPs aggregation

Aptamer stabilized AuNPs resist aggregation

Aptamer stabilized AuNPs Susceptible to aggregation in the presence of aptamer target
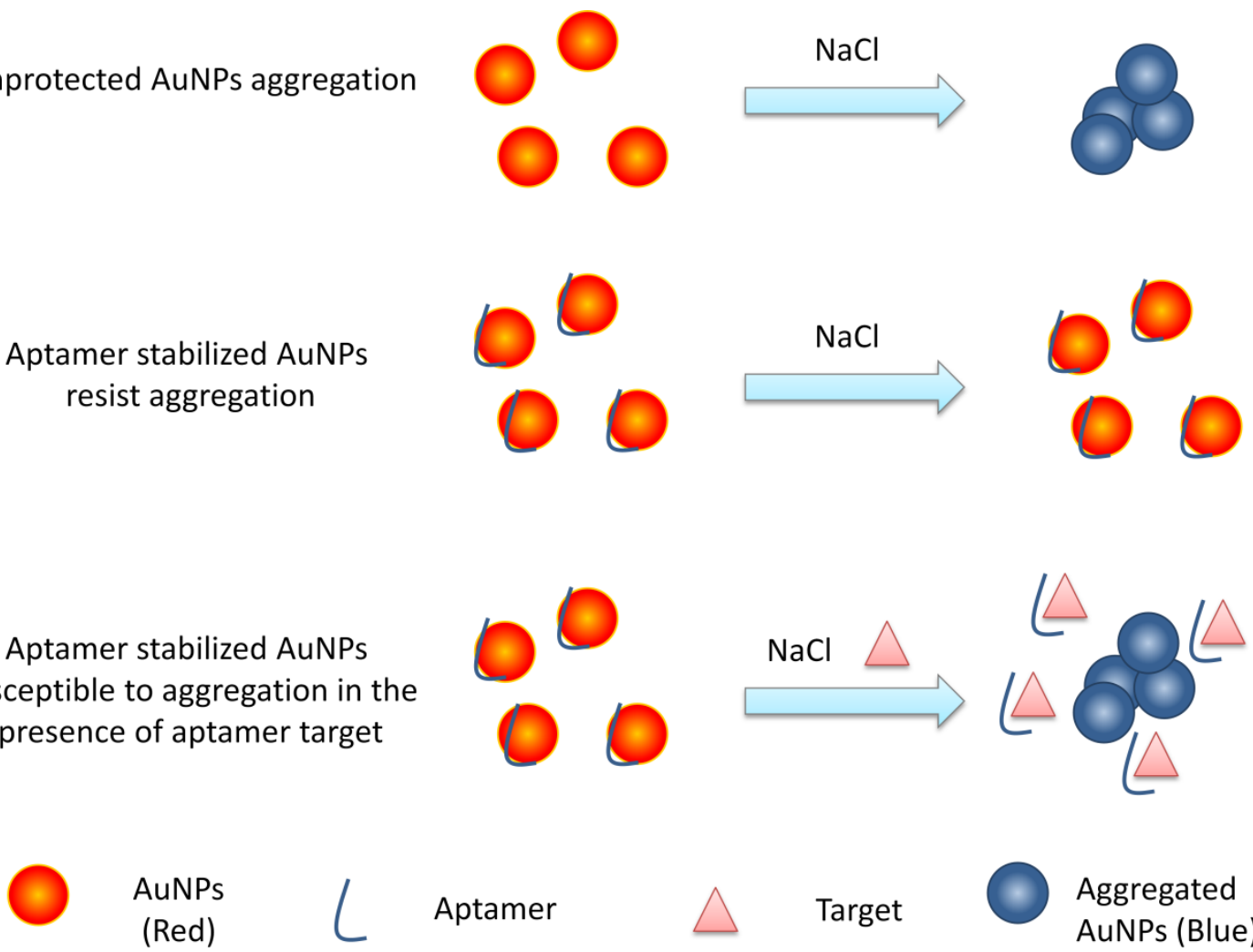

Target

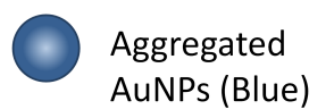

Figure 2.4 A label-free colorimetric aptasensor based on the color change of gold nanoparticles (AuNPs) upon aggregation for biosensing [63].

\subsubsection{Aptamers Applied in Fluorescent Biosensors}

Optical aptasensors have attracted great interest from researchers due to their high sensitivity, simple operation, and rapid detection. Briefly, optical aptasensors utilize signals of colorimetry, fluorescence, chemiluminescence, surface plasmon resonance (SPR), and so on. Among these optical methods, fluorescent signaling is one of the most popular optical approaches for aptamers because of its remarkable sensitivity, high selectivity, high efficiency and easy operation, as well as the unique features of aptamers. As a consequence, various fluorescence-based aptasensors have been developed in biosensing for a wide range of detections: adenosine triphosphate (ATP), ochratoxin A, Cocaine, Adenosine, etc. [20]. Figure 2.5 illustrates a typical graphene oxide-based fluorescence resonance energy transfer (FRET) aptasensor that realized by fluorescence resonance energy transfer [44,68]. This strategy illustrates an example in sensitive fluorescent sensing based on aptamer's conformational change through adaptive binding. 


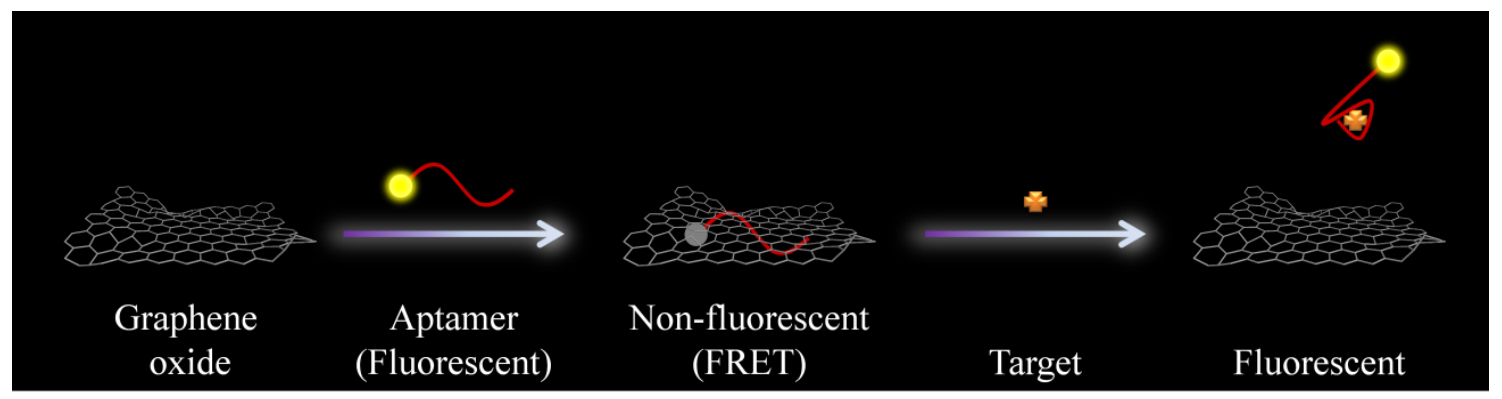

Figure 2.5 Scheme of a typical graphene oxide-based fluorescent aptameric biosensor using fluorescence resonance energy transfer (FRET) [44,68].

\subsubsection{Malachite Green Aptamer (MGA)-A Model Study}

Malachite green aptamer (MGA) was first discovered by Wilson Charles and his lab for site-specific inactivation of RNA transcripts [69] (Figure 2.6). Wilson Charles continued to find that the MGA can not only bind to malachite green (MG) but also bind to some dyes having similar structures as MG, and a crystal structure of MGA bound to tetramethylrosamine (TMR) was obtained [70-72]. When MG binds to MGA, the charge distribution and conformation of MG results in 2000 fold enhancement of MG quantum yield [72-75]. The conformational change in MG or MGA indicates a rearrangement in micro-environment surrounding, which provides possibility of providing an analyte concentration correlation in fluorescent decay kinetics. Based on the unique fluorescence enhancement of MGA on MG, a simple and label-free approach for the determination of binding affinity (or $K_{\mathrm{d}}$ ) can be developed in both direct and competitive binding formats. Fluorescence polarization (FP) may also be employed for $K_{\mathrm{d}}$ determination as MGA can also bind to other fluorescent ligands (small molecules). Immobilized MG was originally used in affinity chromatography to select the MGA, which indicates MGA has ability to bind to MG conjugates, allowing a strategy to conjugate stilbene [70,76]. The successful use of MGA for detection and analysis of MG and leucomalachite green (LMG) residues in aquaculture testifies to its commercial viability [77]. Previous investigations on MGA have detailed the inviolable nucleotides and structure activity relationships required for biosensing [78]. According to the report, the research also compared the 3D crystal (XRay Diffraction, XRD) and solution (nuclear magnetic resonance, NMR) structure of 
MGA, which gives more information of the MGA and give indication on where should be modified. For instance, U4 is away from the binding site, A9 mutant within binding site has tinny impact on $K_{\mathrm{d}}, \mathrm{C} 20$ is close to the tetraloop and C38 is the end modification commonly used. These positions should be considered in our design. On the other hand, MG as an efficient fungicide with commercial prevalence has been banned for fish farming in Canada, the United States and European union owing to its potential toxicity [79]. Detection of MG is in need, and the designed sensor according to our hypothesis (if true) may directly realize a novel commercial assay for the detection of toxin $\mathrm{MG}$.

To summarize, MGA is a model system towards stilbene-based aptasensor development because $K_{\mathrm{d}}$ is easily measured with commercial plate readers, has relative high affinity to MG and TMR analytes, has a known crystal (XRD) and solution structure after binding with MG analyte (NMR), and retains its ability to bind immobilized MG-conjugates.

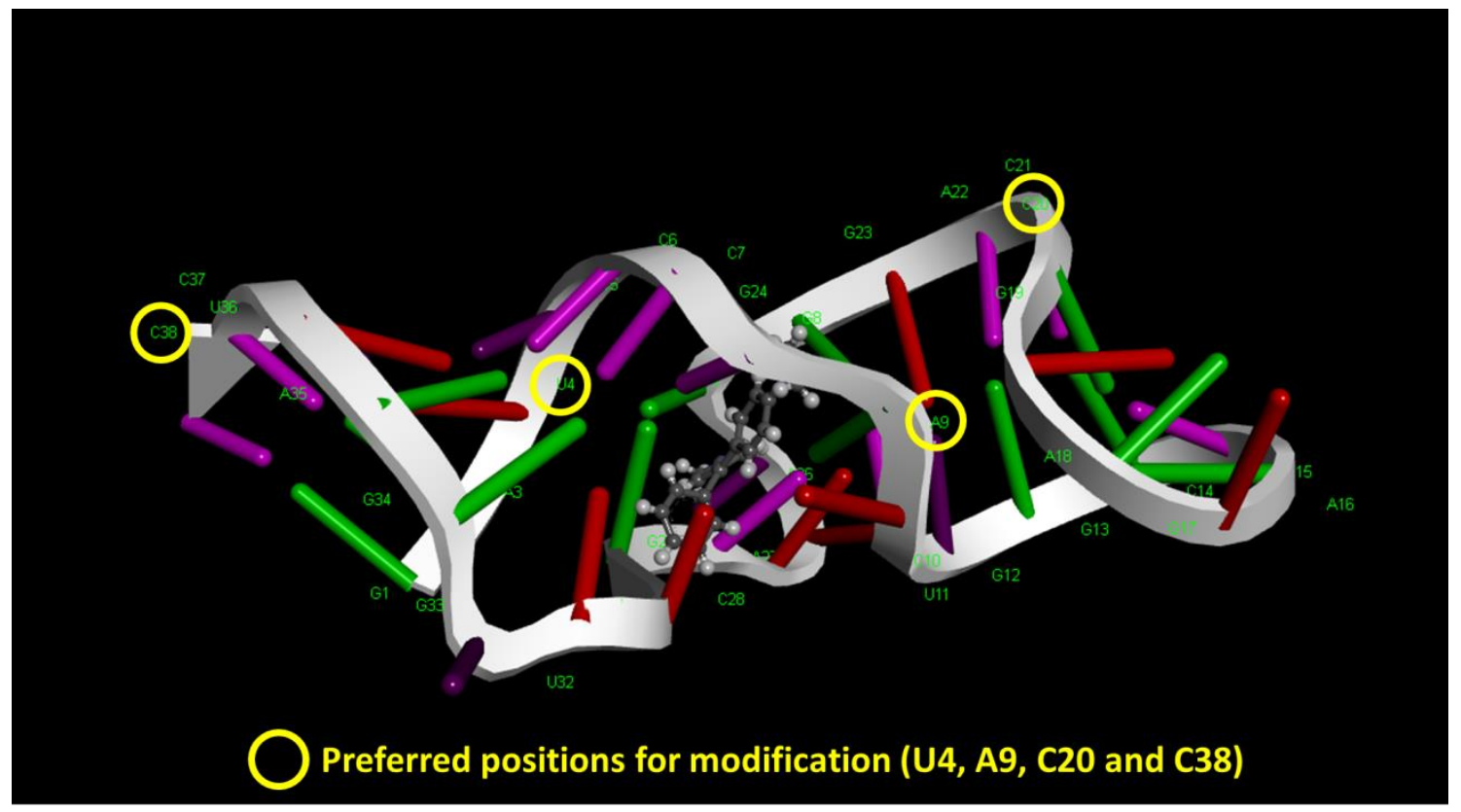

Figure 2.6 Structure of malachite green aptamer (MGA) with MG bound. 3D structure of MGA is presented by Discovery studio 3.5 Visualizer, Accelrys. Nucleic Acid Sequence: 38mer GGAUCCCGACUGGCGAGAGCCAGGUAACGAAUGGAUCC. 3D structure of MGA (1Q8N) is presented by Discovery studio 3.5 Visualizer, Accelrys. Preferred positions for modification are marked according to the $3 \mathrm{D}$ conformation and the structure activity relationship. 


\subsubsection{Summary}

In summary, as discussed above, the significant advantages of aptamers make them become increasingly powerful tools for food safety, environmental monitoring, drug discovery, diagnostics, therapeutics, and so on [37,43,80,81]. MGA with their targets which is well studied should be reasonable for the model study of the stilbene-based aptasensor quantitated by fluorescence decay.

\subsection{Stilbene Compounds-Reporters}

\subsubsection{Current Reporters and Definition of Stilbene}

Fluorescence-based optical reporters are commonly used due to their high sensitivity [14]. Fluorescence reporters which are sensitive, rapid, simple, and cost-effective are the most commonly used reporters in aptamer-based biosensors [22]. However, the fluorescencebased reporters (probes or labels) are now mainly based on the "signal on-off" mode which relies on fluorescence intensity quantification or fluorescence quenching. This mode can be easily affected by various factors, resulting in inaccurate results.

Stilbene is a fluorescent molecule existing in two forms: trans-isomer and cis-isomer. The structures of trans-stilbene and cis-stilbene can be seen in Figure 2.7.

\subsubsection{Fluorescence Properties of Stilbene}

The trans-isomer stilbene is strongly fluorescent, but upon excitation, it undergoes a conformation shift to the cis-isomer which is non-fluorescent. Determination of apparent fluorescence decay rate constant $\left(k_{\mathrm{app}}\right)$ of stilbene molecules has been developed [82] and defined as classical photoisomerization in organic photochemistry [25]. This fluorescence decay may take only seconds to reach a steady state equilibrium, a property we can exploit for rapid sensing. Figure 2.7 shows the typical structures of trans-stilbene and cis-stilbene, and the fluorescence decay of trans-stilbene upon irradiation [24,25]. 
Derivatives of stilbene also show similar fluorescence behavior [83-90] as non-modified stilbene, which makes stilbene have the potential towards sensing micro-environment rearrangements, such as those present in aptamer adaptive binding.

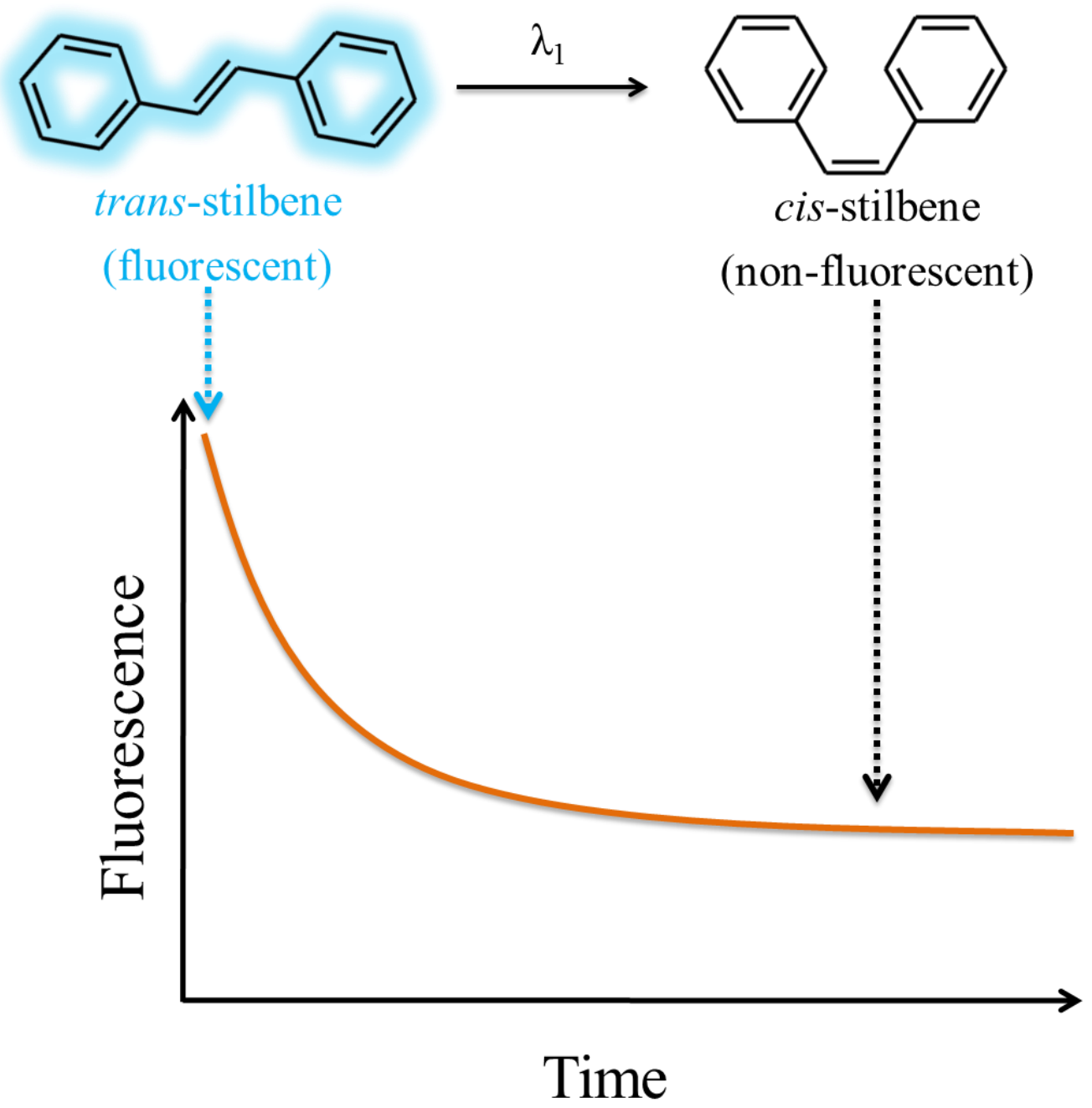

Figure 2.7 Trans-isomer and cis-isomer of stilbene and schematic illustration of the fluorescence decay property of trans-stilbene upon UV irradiation [24,25,91].

\subsubsection{Variation in Photoisomerization of Stilbene Compounds}

The photoisomerization/switching of stilbene kinetics can be affected by factors such as steric hindrance, viscosity, temperature, and solvent. Studies have shown that trans-cis switching of stilbenes can be affected or inhibited by various causes [86,89-91]. Steric 
hindrance or viscous micro-environment is one of the most significant factors of this effect $[89,92]$. When a trans-stilbene molecule was put into a constrained capsule or was enclosed by a large molecule, photodecay kinetics were correlated to the steric hindrance (Figure 2.8) $[88,89,93,94]$. While stilbene derivatives were fixed on protein coated quartz surface, their apparent fluorescence decay rate were times slower than when they were in solution, which also suggested the influence of immobilizing environments. Moreover, this research also shows the impact on the decay rate from absolute viscosity [92]. In the stilbene-based aptasensors, long chain aptamers may also act like a capsule or large molecule to enclose trans-stilbene by "adaptive binding", resulting in constrained micro-environment of stilbene and inhibition of the photoisomerization. This can be correlated to stilbene photodecay rates due to the allosteric effect of aptamers upon binding. Because the stilbene-based aptasensors are based on the fluorescence decay of trans-stilbene, compared to FI-based "signal on/off" sensors it allows separation free detection of bound and unbound targets. These properties of stilbene fluorophores may make stilbene-based aptasensors overcome the shortcomings of the "signal on/off" aptasensors.
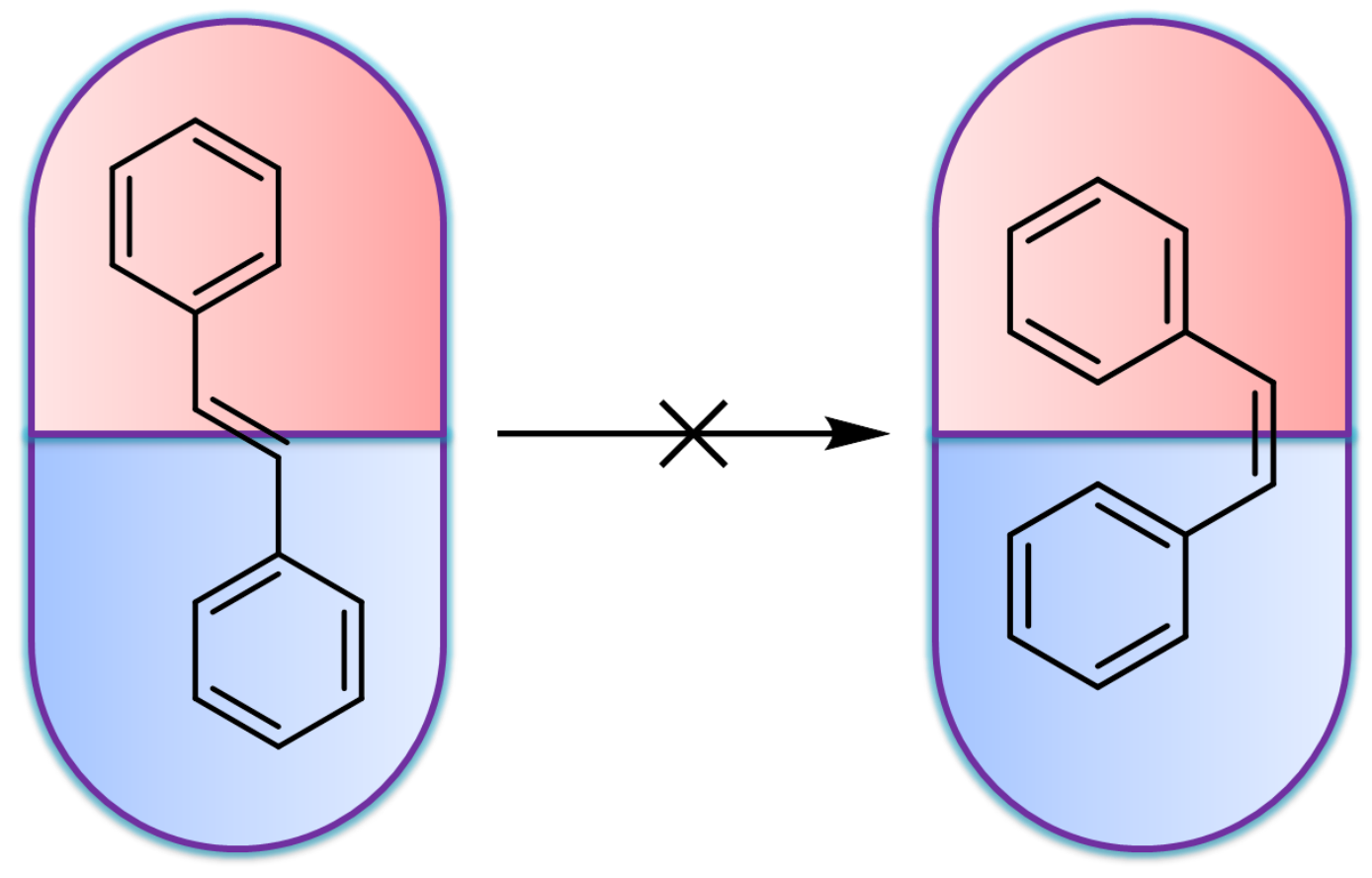

Figure 2.8 Inhibition of stilbene photoisomerization by being placed in an octa acid capsule. Conformations were evidenced by ${ }^{1} \mathrm{H}$ NMR [93]. 


\subsubsection{Regeneration of Decayed Stilbene Compounds}

As sensors, the devices capable of multiple-used analytes and continuous monitoring are welcomed. Thus, a reusable device is prior to a single-used one. The reversible trans-cis switching of stilbene compounds makes it an ideal reporter element in multiple-used sensing. Even though the conversion of trans-stilbene to cis-stilbene is relatively efficient, the cis form can be turned back to trans form under certain condition, e.g. UV-Vis irradiation in another wavelength [95]. Studies show that this "turning back" conformational change makes stilbene molecule recover fluorescent to almost the initial level [86]. This regeneration ability of stilbene combining with the reversible allostery of aptamer makes them an ideal couple of sensing element which may have a lower cost of detection compared to the disposable elements. Figure 2.9 gives an example of the fluorescence change of stilbene derivative from cis form to trans form [86].

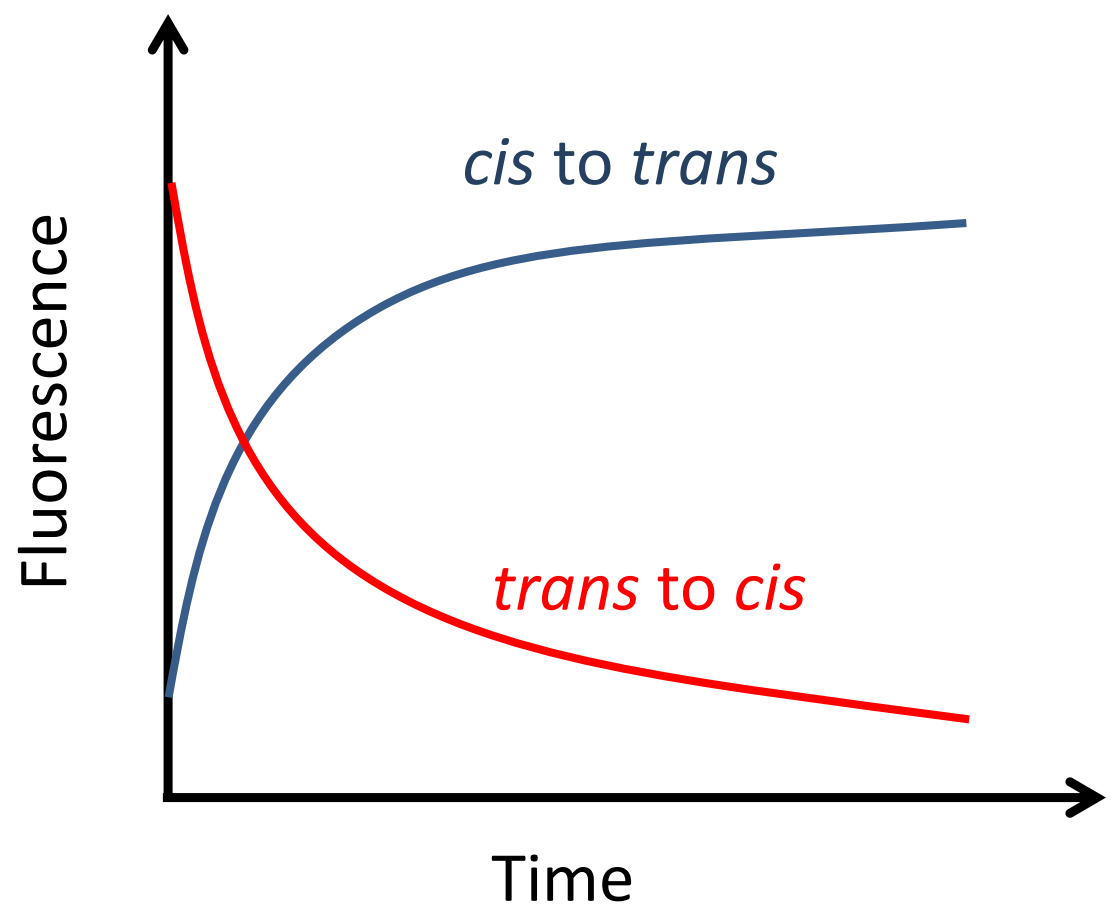

Figure 2.9 Schematic illustration of fluorescence change of stilbene upon photoisomerization. Trans-cis: excitation $350 \mathrm{~nm}$, emission $438 \mathrm{~nm}$, and cis-trans: excitation $300 \mathrm{~nm}$, emission 438 $\mathrm{nm}$. Graph is redrawn from reference [86]. 


\subsubsection{Azobenzenes}

Besides stilbenes, there are diverse organic photochromic molecules undergoing switching upon activation: azobenzenes, fulgides, diarylethenes, chromenes, spiropyranes and so on [96]. Specifically, azobenzenes are structurally similar to stilbenes (with $-\mathrm{N}=\mathrm{N}-$ instead of $-\mathrm{C}=\mathrm{C}$ - compared to stilbene). Figure 2.10A displays the structures of transazobenzene and cis-azobenzene and their photoisomerization. The time for trans-to-cis isomerization of azobenzenes is in picosecond or even femtosecond scale, while the time for stilbenes is mainly in picosecond scale [96-99]. This difference on azobenzenes can be applied while faster signal reporting is needed. However, it should be noted that the fluorescence quantum yield of azobenzenes is 3-5 orders smaller than the fluorescence quantum yield of stilbenes, which limits their fluorescence-based applications [100-103]. Finally, as another type of stilbene-like compounds, $N$-benzylideneaniline (Figure 2.10B) compounds (with $-\mathrm{C}=\mathrm{N}$ - instead of $-\mathrm{C}=\mathrm{C}$ - compared to stilbene) also possess the property of isomerization upon UV excitation, having merits of convenient synthesis and lower activation energy [104]. However, $N$-benzylideneaniline compounds are rarely used for photoswitch sensing because of the easy hydrolysis of imines [105].

A

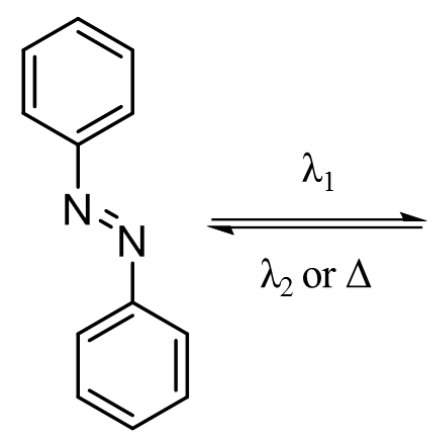

B<smiles>c1ccc(/N=N/c2ccccc2)cc1</smiles>

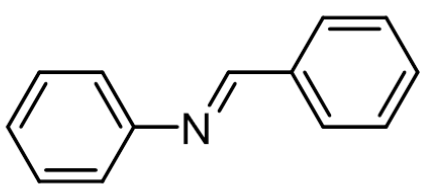

Figure 2.10 Chemical structures of stilbene-like compounds. (A) Trans-isomer and cis-isomer of azobenzene. (B) $N$-benzylideneaniline.

\subsubsection{Summary}

In summary, taking the discussion above into account, stilbene and its derivatives have the excellent features which fulfill the requirements of an ideal sensing element for our 
design. The high photochemical sensitivity, permission to be modified, fast response and the ability of regeneration make stilbene a promising reporter in sensing. Other stilbenelike compounds are also reviewed. Azobenzenes are possible substitutes of stilbenes for rapid assay if their fluorescence quantum yield can be sufficiently enhanced. $\mathrm{N}$ benzylideneaniline compounds are hardly used due to the easy hydrolysis of imines.

\section{References:}

[1] C. R. Lowe Biosensors 1985, 1, 3.

[2] H. Sharma; R. Mutharasan Sensors and Actuators B: Chemical 2013, 183, 535.

[3] V. C. Rucker; K. L. Havenstrite; A. E. Herr Analytical Biochemistry 2005, 339, 262.

[4] A. Rasooly; K. E. Herold Journal of AOAC International 2006, 89, 873.

[5] S. Rodriguez-Mozaz; M. J. Lopez de Alda; D. Barceló Analytical and Bioanalytical Chemistry 2006, 386, 1025.

[6] H. Hasegawa; K.-i. Taira; K. Sode; K. Ikebukuro Sensors 2008, 8, 1090.

[7] K. Narsaiah; S. N. Jha; R. Bhardwaj; R. Sharma; R. Kumar Journal of Food Science and Technology 2011, 49, 383.

[8] A. P. F. Turner Chemical Society Reviews 2013, 42, 3184.

[9] S. P. Mohanty; E. Kougianos IEEE Potentials 2006, 25, 35.

[10] L. D. Mello; L. T. Kubota Food Chemistry 2002, 77, 237.

[11] M. Pietrzak In Reference Module in Chemistry, Molecular Sciences and Chemical Engineering; Elsevier: 2015.

[12] T. H. Rider; M. S. Petrovick; F. E. Nargi; J. D. Harper; E. D. Schwoebel; R. H. Mathews; D. J. Blanchard; L. T. Bortolin; A. M. Young; J. Chen; M. A. Hollis Science 2003, 301, 213.

[13] S. K. Arya; A. Singh; R. Naidoo; P. Wu; M. T. McDermott; S. Evoy Analyst 2011, $136,486$.

[14] R. Monošík; M. Stred'anský; E. Šturdík In Acta Chimica Slovaca 2012; Vol. 5, p 109.

[15] P.-Y. Chen; R. Vittal; P.-C. Nien; G.-S. Liou; K.-C. Ho Talanta 2010, 80, 1145.

[16] R. Monošík; M. Stred'anský; E. Šturdík Journal of Clinical Laboratory Analysis 2012, 26, 22. 
[17] S. Y. Toh; M. Citartan; S. C. B. Gopinath; T.-H. Tang Biosensors and Bioelectronics 2015, 64, 392.

[18] K.-M. Song; S. Lee; C. Ban Sensors 2012, 12, 612.

[19] J. W. Ndieyira; N. Kappeler; S. Logan; M. A. Cooper; C. Abell; R. A. McKendry; G. Aeppli Nat Nano 2014, 9, 225.

[20] C. Feng; S. Dai; L. Wang Biosensors and Bioelectronics 2014, 59, 64.

[21] J. Liu; Z. Cao; Y. Lu Chemical Reviews 2009, 109, 1948.

[22] R. E. Wang; Y. Zhang; J. Cai; W. Cai; T. Gao Current Medicinal Chemistry 2011, 18,4175 .

[23] J. L. Vinkenborg; N. Karnowski; M. Famulok Nat Chem Biol 2011, 7, 519.

[24] F. B. Mallory; C. S. Wood; J. T. Gordon; L. C. Lindquist; M. L. Savitz Journal of the American Chemical Society 1962, 84, 4361.

[25] D. H. Waldeck Chemical Reviews 1991, 91, 415.

[26] A. Meir; R. S. Marks; M. N. Stojanovic In Handbook of Biosensors and Biochips; John Wiley \& Sons, Ltd: 2008.

[27] A. D. Ellington; J. W. Szostak Nature 1990, 346, 818.

[28] C. Tuerk; S. Eddy; D. Parma; L. Gold Journal of Molecular Biology 1990, 213, 749.

[29] D. S. Wilson.; J. W. Szostak Annual Review of Biochemistry 1999, 68, 611.

[30] S. L. Clark; V. T. Remcho ELECTROPHORESIS 2002, 23, 1335.

[31] S. Tombelli; M. Minunni; M. Mascini Biosensors and Bioelectronics 2005, 20, 2424.

[32] C. L. A. Hamula; J. W. Guthrie; H. Zhang; X.-F. Li; X. C. Le TrAC Trends in Analytical Chemistry 2006, 25, 681.

[33] E. Peyrin Journal of Separation Science 2009, 32, 1531.

[34] T. Šmuc; I.-Y. Ahn; H. Ulrich Journal of Pharmaceutical and Biomedical Analysis 2013, 81-82, 210 .

[35] A. B. Iliuk; L. Hu; W. A. Tao Analytical Chemistry 2011, 83, 4440.

[36] H. Ulrich; C. A. Trujillo; A. A. Nery; J. M. Alves; P. Majumder; R. R. Resende; A. H. Martins Comb Chem High Throughput Screen 2006, 9, 619.

[37] S. Song; L. Wang; J. Li; C. Fan; J. Zhao TrAC Trends in Analytical Chemistry 2008, 27, 108.

[38] A. A. Nery; C. Wrenger; H. Ulrich Journal of Separation Science 2009, 32, 1523. 
[39] Y. Du; B. Li; E. Wang Accounts of Chemical Research 2013, 46, 203.

[40] J. T. H. Yeh; R. Binari; T. Gocha; R. Dasgupta; N. Perrimon Scientific Reports 2013, 3,1156 .

[41] S. D. Jayasena Clinical Chemistry 1999, 45, 1628.

[42] M. Famulok; G. Mayer Accounts of Chemical Research 2011, 44, 1349.

[43] T. Hermann; D. J. Patel Science 2000, 287, 820.

[44] J. Ping; Y. Zhou; Y. Wu; V. Papper; S. Boujday; R. S. Marks; T. W. J. Steele Biosensors and Bioelectronics 2015, 64, 373.

[45] B. Strehlitz; R. Stoltenburg In Aptamers in Bioanalysis; John Wiley \& Sons, Inc.: 2008, p 31 .

[46] D. Li; S. Song; C. Fan Accounts of Chemical Research 2010, 43, 631.

[47] O. Reinstein; M. A. D. Neves; M. Saad; S. N. Boodram; S. Lombardo; S. A. Beckham; J. Brouwer; G. F. Audette; P. Groves; M. C. J. Wilce; P. E. Johnson Biochemistry 2011, 50, 9368.

[48] M. A. D. Neves; C. Blaszykowski; M. Thompson Analytical Chemistry 2016, 88, 3098.

[49] S. Xie; S. P. Walton Analytica Chimica Acta 2009, 638, 213.

[50] J.-W. Chen; X.-P. Liu; K.-J. Feng; Y. Liang; J.-H. Jiang; G.-L. Shen; R.-Q. Yu Biosensors and Bioelectronics 2008, 24, 66.

[51] J. Zhang; X. Zhang; G. Yang; J. Chen; S. Wang Biosensors and Bioelectronics 2013, $41,704$.

[52] Q. Zhao; X. Geng; H. Wang Analytical and Bioanalytical Chemistry 2013, 405, 6281.

[53] W. Zhou; P.-J. Jimmy Huang; J. Ding; J. Liu Analyst 2014, 139, 2627.

[54] F. Le Floch; H. A. Ho; M. Leclerc Analytical Chemistry 2006, 78, 4727.

[55] M. Kuwahara; N. Sugimoto Molecules 2010, 15, 5423.

[56] S. M. Nimjee; C. P. Rusconi; B. A. Sullenger Annual Review of Medicine 2005, 56, 555.

[57] J.-O. Lee; H.-M. So; E.-K. Jeon; H. Chang; K. Won; Y. H. Kim Analytical and Bioanalytical Chemistry 2007, 390, 1023. 
[58] K. Ikebukuro; W. Yoshida; K. Sode In Aptamers in Bioanalysis; John Wiley \& Sons, Inc.: 2008, p 129.

[59] M. Jing; M. T. Bowser Analytica Chimica Acta 2011, 686, 9.

[60] P. Hong; W. Li; J. Li Sensors 2012, 12, 1181.

[61] C. Hart Journal of Biomolecular Screening 2013, 18, 1138.

[62] N. O. Fischer; T. M. Tarasow; J. B. H. Tok Current Opinion in Chemical Biology 2007, 11, 316 .

[63] N. Derbyshire; S. J. White; D. H. J. Bunka; L. Song; S. Stead; J. Tarbin; M. Sharman; D. Zhou; P. G. Stockley Analytical Chemistry 2012, 84, 6595.

[64] K.-M. Song; M. Cho; H. Jo; K. Min; S. H. Jeon; T. Kim; M. S. Han; J. K. Ku; C. Ban Analytical Biochemistry 2011, 415, 175.

[65] C. Yang; Y. Wang; J.-L. Marty; X. Yang Biosensors and Bioelectronics 2011, 26, 2724.

[66] A. D. Keefe; S. Pai; A. Ellington Nat Rev Drug Discov 2010, 9, 537.

[67] J. H. Soh; Y. Lin; S. Rana; J. Y. Ying; M. M. Stevens Analytical Chemistry 2015, 87, 7644.

[68] C.-H. Lu; J. Li; M.-H. Lin; Y.-W. Wang; H.-H. Yang; X. Chen; G.-N. Chen Angewandte Chemie International Edition 2010, 49, 8454.

[69] D. Grate; C. Wilson Proceedings of the National Academy of Sciences 1999, 96, 6131.

[70] C. Baugh; D. Grate; C. Wilson Journal of Molecular Biology 2000, 301, 117.

[71] J. Bernard Da Costa; T. Dieckmann Molecular BioSystems 2011, 7, 2156.

[72] J. B. Da Costa; A. I. Andreiev; T. Dieckmann Biochemistry 2013, 52, 6575.

[73] D. H. Nguyen; S. C. DeFina; W. H. Fink; T. Dieckmann Journal of the American Chemical Society 2002, 124, 15081.

[74] J. R. Babendure; S. R. Adams; R. Y. Tsien Journal of the American Chemical Society 2003, 125, 14716.

[75] D. H. Nguyen; T. Dieckmann; M. E. Colvin; W. H. Fink The Journal of Physical Chemistry B 2004, 108, 1279. 
[76] M. Hirabayashi; S. Taira; S. Kobayashi; K. Konishi; K. Katoh; Y. Hiratsuka; M. Kodaka; T. Q. P. Uyeda; N. Yumoto; T. Kubo Biotechnology and Bioengineering 2006, $94,473$.

[77] S. L. Stead; H. Ashwin; B. H. Johnston; A. Dallas; S. A. Kazakov; J. A. Tarbin; M. Sharman; J. Kay; B. J. Keely Analytical Chemistry 2010, 82, 2652.

[78] J. Flinders; S. C. DeFina; D. M. Brackett; C. Baugh; C. Wilson; T. Dieckmann ChemBioChem 2004, 5, 62.

[79] W. C. Andersen; S. B. Turnipseed; C. M. Karbiwnyk; R. H. Lee; S. B. Clark; W. D. Rowe; M. R. Madson; K. E. Miller Analytica Chimica Acta 2009, 637, 279.

[80] K. Thiel Nat Biotech 2004, 22, 649.

[81] S. Tombelli; M. Minunni; M. Mascini Biomolecular Engineering 2007, 24, 191.

[82] V. Papper; G. I. Likhtenshtein; N. Medvedeva; D. V. Khoudyakov Journal of Photochemistry and Photobiology A: Chemistry 1999, 122, 79.

[83] G. I. Likhtenshtein; R. Bishara; V. Papper; B. Uzan; I. Fishov; D. Gill; A. H. Parola Journal of Biochemical and Biophysical Methods 1996, 33, 117.

[84] A. Simeonov; M. Matsushita; E. A. Juban; E. H. Z. Thompson; T. Z. Hoffman; A. E. Beuscher IV; M. J. Taylor; P. Wirsching; W. Rettig; J. K. McCusker; R. C. Stevens; D. P. Millar; P. G. Schultz; R. A. Lerner; K. D. Janda Science 2000, 290, 307.

[85] H. Meier Angewandte Chemie International Edition 2001, 40, 1851.

[86] O. Chen; R. Glaser; G. I. Likhtenshtein Biophysical Chemistry 2003, 103, 139.

[87] H. Matsushita; N. Yamamoto; M. M. Meijler; P. Wirsching; R. A. Lerner; M. Matsushita; K. D. Janda Molecular BioSystems 2005, 1, 303.

[88] E. W. Debler; G. F. Kaufmann; M. M. Meijler; A. Heine; J. M. Mee; G. Pljevaljčić; A. J. Di Bilio; P. G. Schultz; D. P. Millar; K. D. Janda; I. A. Wilson; H. B. Gray; R. A. Lerner Science 2008, 319, 1232.

[89] M. R. Ams; D. Ajami; S. L. Craig; J.-S. Yang; J. Rebek, Jr. Beilstein Journal of Organic Chemistry 2009, 5, 79.

[90] L. M. Tolbert; A. Baldridge; J. Kowalik; K. M. Solntsev Accounts of Chemical Research 2012, 45, 171.

[91] O. Chen; R. Glaser; G. I. Likhtenshtein Journal of Biochemical and Biophysical Methods 2008, 70, 1073. 
[92] N. Strashnikova; V. Papper; P. Parkhomyuk; G. I. Likhtenshtein; V. Ratner; R. Marks Journal of Photochemistry and Photobiology A: Chemistry 1999, 122, 133.

[93] A. Parthasarathy; L. S. Kaanumalle; V. Ramamurthy Organic Letters 2007, 9, 5059.

[94] D. Tzeli; G. Theodorakopoulos; I. D. Petsalakis; D. Ajami; J. Rebek Journal of the American Chemical Society 2012, 134, 4346.

[95] F. B. Mallory; C. W. Mallory Journal of the American Chemical Society 1972, 94, 6041.

[96] J. García-Amorós; D. Velasco Beilstein Journal of Organic Chemistry 2012, 8, 1003.

[97] A. A. Beharry; G. A. Woolley Chemical Society Reviews 2011, 40, 4422.

[98] S. A. Kovalenko; A. L. Dobryakov Chemical Physics Letters 2013, 570, 56.

[99] C. Jiang; R. Xie; F. Li; R. E. Allen Chemical Physics Letters 2009, 474, 263.

[100] J. L. Charlton; J. Saltiel The Journal of Physical Chemistry 1977, 81, 1940.

[101] H. Satzger; S. Spörlein; C. Root; J. Wachtveitl; W. Zinth; P. Gilch Chemical Physics Letters 2003, 372, 216.

[102] M. R. Han; Y. Hirayama; M. Hara Chemistry of Materials 2006, 18, 2784.

[103] M. Han; D. Ishikawa; E. Muto; M. Hara Journal of Luminescence 2009, 129, 1163.

[104] Y. Luo, Freie Universität Berlin, 2011.

[105] N. A. Alexander, University of Canterbury, 2006. 


\section{Chapter 3}

\section{Experimental Methodology}

This chapter introduces the main experimental methods and corresponding principles used in this thesis. Section 3.1 describes briefly the rationale of selection of the methods. Section 3.2 explains the reason for using the model aptamer-malachite green aptamer (MGA). Section 3.3 introduces the highthroughput technique and principle in determination of aptamer binding affinity. Section 3.4 shows the measurement of stilbene fluorescence emission and decay spectra. Section 3.5 is about the techniques used in synthesis and characterization of aptamer-stilbene conjugate. Strategy on monitoring the kinetics of MG conversion is described in section 3.6. Additionally, methods employed for statistical analysis are also introduced in section 3.7. This chapter mainly focuses on the rationale of methods chosen in our experiments, with examples given to show the rationality and appropriateness of such methods. Detailed experimental procedures will be introduced in Result chapters. 


\subsection{Rationale for selection}

To test the hypothesis, an appropriate model aptamer needs to be selected foremost. Our designs require an ideal aptamer to be as small as possible, nM binding affinity $\left(K_{\mathrm{d}}\right.$ less than $1 \mu \mathrm{M}$ ), fast $K_{\mathrm{d}}$ determination, amenable in modification and known 3D structure upon binding. Malachite green aptamer (MGA)/ malachite green (MG) as aptamer/ligand pair is chosen as an ideal model aptamer system as it possesses all the properties required and additionally has advantages beneficial for the designs within the scopes in this thesis, such as the fluorescence enhancement of MG upon binding to MGA towards rapid $K_{\mathrm{d}}$ assessment. Binding affinity determination based on fluorescence enhancement of MG allows using minimal amounts of MGA which is the most expensive consumable in our project. Shimadzu RF-5301PC Spectrofluorophotometer providing continuous fluorescent excitation is employed in the fluorescence emission and decay spectra measurement of stilbene compounds while high-throughput TECAN M200 microplate reader can only supply pulse excitation, which is not applicable for stilbene excitation. In the synthesis of aptamer-stilbene conjugate, amine modified MGA and 4-Acetamido-4'isothiocyanato-2,2'-stilbenedisulfonic acid disodium salt (SITS) are selected which are commercially available. Additionally, SITS which has significant fluorescence decay in aqueous system is a preferred choice for our experimental design. Finally, for characterization of MGA-SITS conjugate, matrix-assisted laser desorption/ionization time-of-flight (MALDI-TOF) mass spectrometry is applied to assess the molecular weight of MGA before and after conjugation, while high-performance liquid chromatography-diode array detector (HPLC-DAD) is used for indication of conjugation and purity monitoring. Ultraviolet-visible (UV-Vis) measurement is chosen for studies on kinetics of MG conversion based on the significant UV-Vis absorbance and the known conversion of MG. Methods chosen for statistical analysis in this thesis are the commonly used approaches in data explanation.

\subsection{Selection of Model Aptamer - Malachite Green Aptamer (MGA) and Malachite Green (MG)}


To test our hypothesis, an appropriate aptamer/ligand model possessing the ideal properties is preferred: as small length of nucleotide as possible, binding to small molecules with high affinity $\left(K_{\mathrm{d}}\right.$ less than $\left.1 \mu \mathrm{M}\right)$, ease of binding affinity determination, amendable for modification and well-known 3D NMR or crystal structure. After searching in an online aptamer database (http://aptamer.icmb.utexas.edu/) established by Ellington and co-workers [1], MGA/MG that meets the requirements above has been chosen as an ideal model for our study. There are also other aptamers meet most of the requirements above but ultimately ruled out, which can be considered as candidates in the future. For instance, kanamycin B aptamer has a $K_{\mathrm{d}}$ of $180 \mathrm{nM}$, but its length is more than 100 nucleotides, dramatically increasing the cost on aptamer. A 29-mer sulforhodamine B aptamer binds to sulforhodamine B with a $K_{\mathrm{d}}$ of $190 \mathrm{nM}$, but no report of its NMR or XRD structure is found. Figure 3.1 displays the structures of MGA and MG (1). Besides the properties above, MGA can also bind to other targets such as tetramethylrosamine (TMR), and thus TMR can be a candidate or positive control in our hypothesis testing. The XRD (1F1T) and NMR (1Q8N) structures of MGA has been revealed and used in computational modelling [2,3], which explains the position and interaction between MGA and stilbene molecules. Binding affinities of the MGA point mutations have also been reported [3], which give indications on the modification of MGA in our designs. For example, A9 mutant (within binding site) has tinny impact on $K_{\mathrm{d}}(<2$ times increase in $K_{\mathrm{d}}$ when A9 was replaced by C9). Commercially available derivatives of MG facilitate the synthesis of MG-stilbene conjugates. On the other hand, MG has commercial prevalence as an efficient fungicide but has been banned for fish farming in the United States, Canada and European union due to its potential toxicity [4]. Successful development of an aptasensor according to the hypothesis will provide a new approach for detection of MG. However, it also needs to be noted that MGA is a RNA aptamer, which has a more costly synthesis than DNA. 


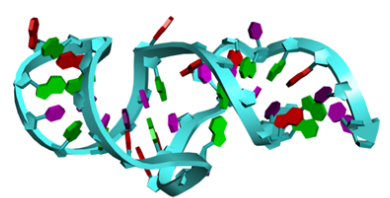

Malachite green aptamer (MGA) 38 nucleotides, PubMed ID: 1Q8N

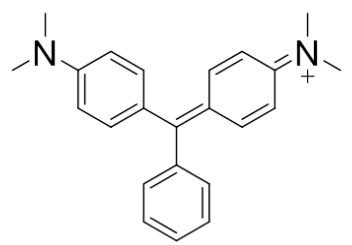

(1)

Malachite green (MG)



(2)

MG- $\mathrm{CH}_{2}-\mathrm{NH}$-stilbene-OMe

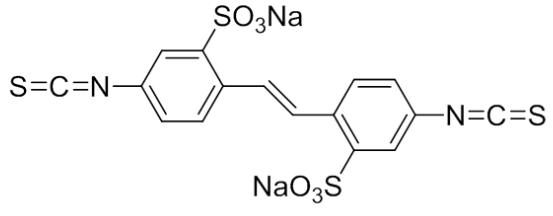

(3)

4,4'-Diisothiocyanatostilbene-2,2'disulfonic acid disodium salt (DIDS)

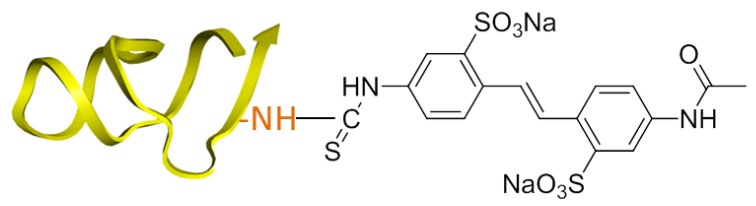

(5)

MGA-SITS conjugate

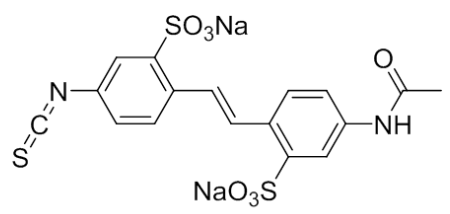

(4)

4-Acetamido-4'-isothiocyanato-2,2'stilbenedisulfonic acid disodium salt (SITS)<smiles>CN(C)c1ccc(C(O)(c2ccccc2)c2ccc(N(C)C)cc2)cc1</smiles>

(6)

Malachite green carbinol base $(\mathrm{MGOH})$

Figure 3.1 Structure of malachite green aptamer (MGA), malachite green (MG) (1), MG$\mathrm{CH}_{2}$-NH-stilbene-OMe (2), 4,4'-Diisothiocyanatostilbene-2,2'-disulfonic acid disodium salt (DIDS) (3), 4-Acetamido-4'-isothiocyanato-2,2'-stilbenedisulfonic acid disodium salt (SITS) (4), MGA-SITS (5) and malachite green carbinol base (MGOH) (6). 3D structure of MGA (1Q8N) is presented by Discovery studio 3.5 Visualizer, Accelrys.

\subsection{Determination of MGA Binding Affinity by High-Throughput Microplate} Assay

\subsubsection{High-Throughput Binding by Fluorescence Enhancement of MG}

There are various techniques used for binding affinity measurements. For instance, fluorescence intensity (FI), fluorescence polarization (FP), ultraviolet-visible (UV-Vis) absorption, surface plasmon resonance (SPR) and isothermal titration calorimetry (ITC) 
are commonly applied in $K_{\mathrm{d}}$ determination [5]. FI method usually utilizes the enhancement or quenching in fluorescence of ligand or aptamer (fluorophore-labelled) upon binding, which is a rapid, sensitive and simple method. However, it requires special properties of ligand or modification of aptamer. FP utilizes the difference in anisotropy of emission light for distinguishing the bound or free fluorescent molecules, which displays different rotational relaxation time. FP is available for binding assay of various fluorescent molecules [6]. However, it has disadvantages like requiring fluorescent/fluorophore-labelled molecules and suitable size of ligands. SPR is a powerful technique for determination for binding affinity based on changes in refractive index, which can perform sensitive, label-free and real-time monitoring. However, this method requires relatively complicated immobilization steps and suffers interference from non-specific binding [7]. ITC is a label-free technique requires no immobilization, but at least $1 \mathrm{~mL}$ of samples is needed in ITC measurements (at least 10 times more than FI method under our experimental conditions), which is not suitable for aptamer binding affinities. According to the methods described above, the simple, sensitive and rapid FI method based on the fluorescence enhancement of MG is selected for binding affinity determination. This method has been developed by us to be a high-throughput approach with a common microplate reader, which is an ideal option for our $K_{\mathrm{d}}$ determination requiring small sample consumption.

Fluorescent technique has been widely used in various research fields such as biological, chemical and medical studies. Fluorescence process includes excitation and subsequent emission of light in a further wavelength. At ground state, a fluorophore can absorb energy of a specific wavelength after is excited by light, and then stays in excited state for a finite time with partially energy dissipation, and finally returns to ground state with light emission in a certain wavelength. The processes are illustrated by the simple electronic-state diagram (Jablonski diagram) in Figure 3.2. Fluorescence possesses advantages such as high sensitivity, high specificity, wide concentration range, relative simplicity and low time consumption, while it also has shortcomings like suffering interferences from impurities, bubbles or environment $(\mathrm{pH}$, temperature, etc). The obvious advantages of fluorescence enable it to be a powerful tool for our MGA/MG 
binding assay which will be described below.

Free non-planar MG has fluorescence quantum yield of $0.790 \times 10^{-4}$ without aptamer binding. However, when MG binds to MGA, it can lead to a unique enhanced quantum yield to 0.187 because of the more planar structure of MG upon binding, which results in significant enhancement in fluorescence ( 2000X) [8]. This fluorescence enhancement can be utilized for monitoring the MGA/MG binding event and measuring the $K_{\mathrm{d}}$ in our study. Figure 3.3 displays the fluorescence enhancement of $\mathrm{MG}$ at $\sim 650 \mathrm{~nm}$ upon binding to MGA.

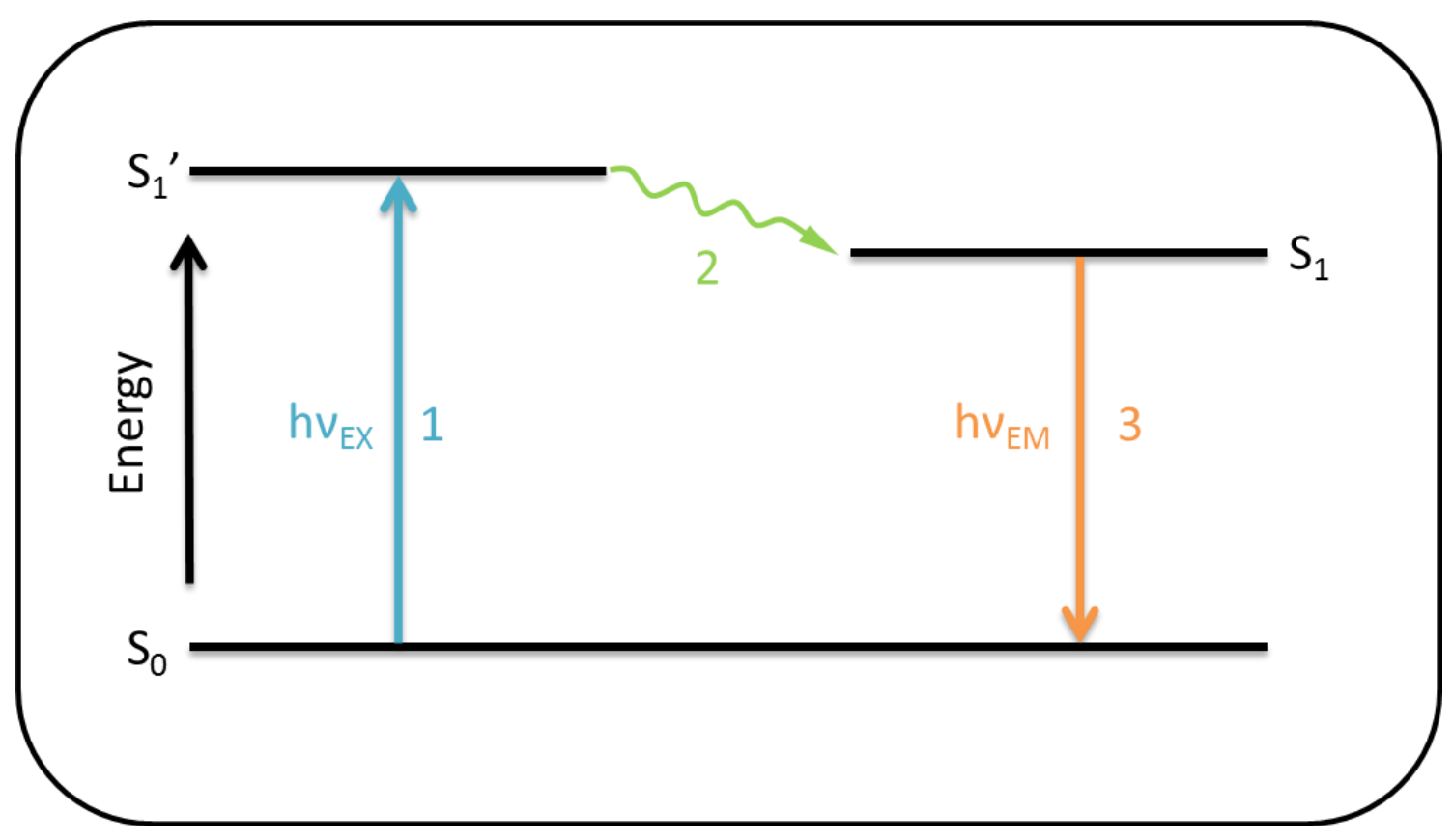

Figure 3.2 Jablonski diagram illustration of fluorescence emission. 


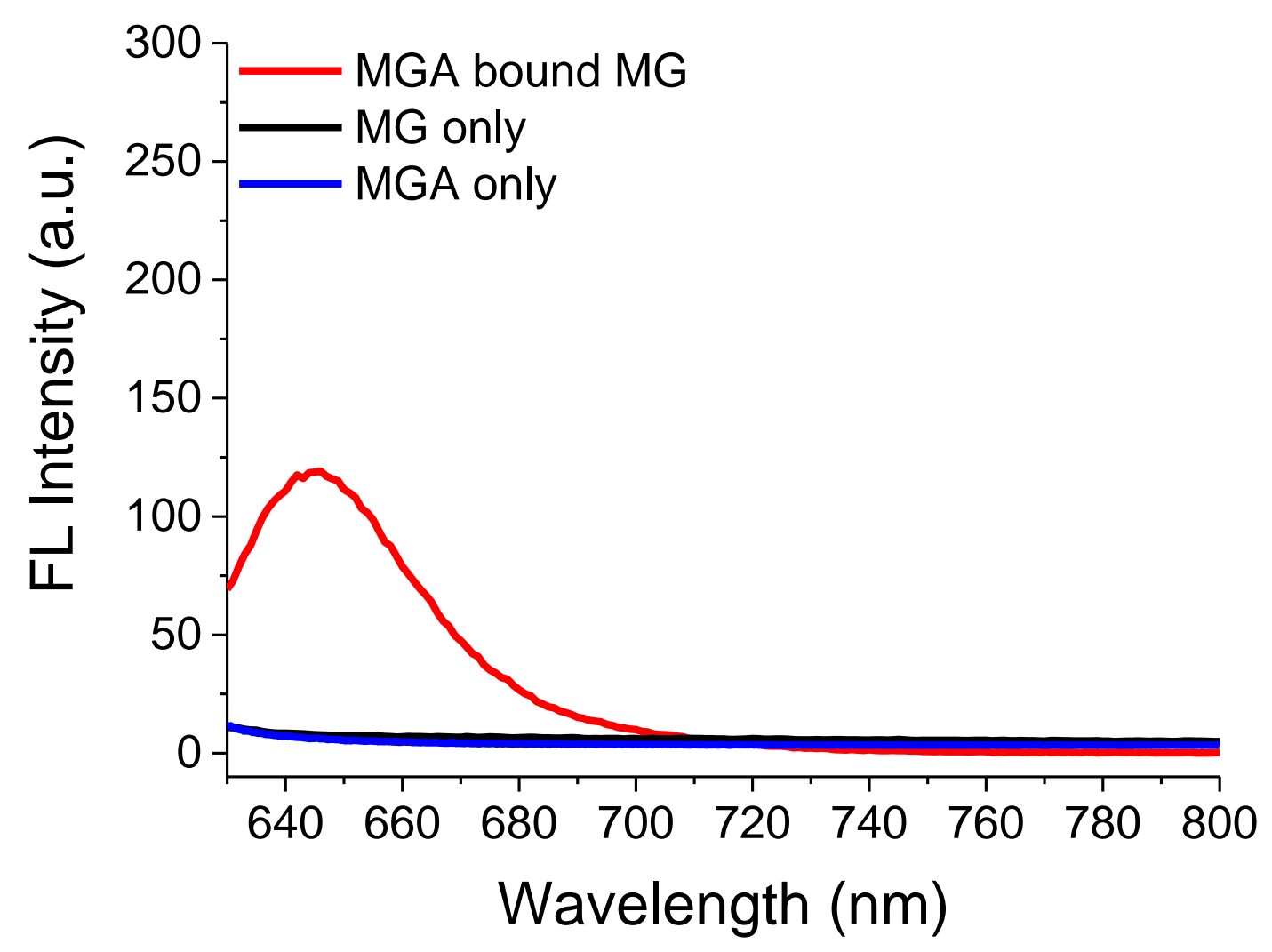

Figure 3.3 Fluorescence enhancement of MG upon binding to MGA at excitation wavelength of $620 \mathrm{~nm}$.

MG derivatives bind to MGA or MGA derivatives bind to MG with small $K_{\mathrm{d}}$ (less than 1 $\mu \mathrm{M})$ is a necessary parameter for success biosensor evaluation, along with specific fluorescence decay properties of stilbene conjugates. This necessitates a high-throughput and rapid binding assay with minimal amounts of aptamer consumption. Fluorescence measurements in 96-well microplate by TECAN M200 microplate reader meet the requirements above. In brief, a typical assay measurement using $150 \mathrm{nM}$ MG with various ratios of MGA in binding buffer are mixed and transferred into a black 96-well microplate and measured by a Tecan Microplate spectrofluorometer (Infinite M200, Tecan Asia PTE LTD, Singapore), triplicate for each concentration. Excitation/emission wavelengths are set to $620 \mathrm{~nm} / 656 \mathrm{~nm}$ [9]. By using this high-throughput fluorescence method, only 5.5 ng of MG is needed for each well. Figure 3.4 is representative data of MG fluorescence enhancement upon binding to MGA, where a saturation of MGA is obtained after $\sim 10 \mathrm{X}$ of MGA is present. 


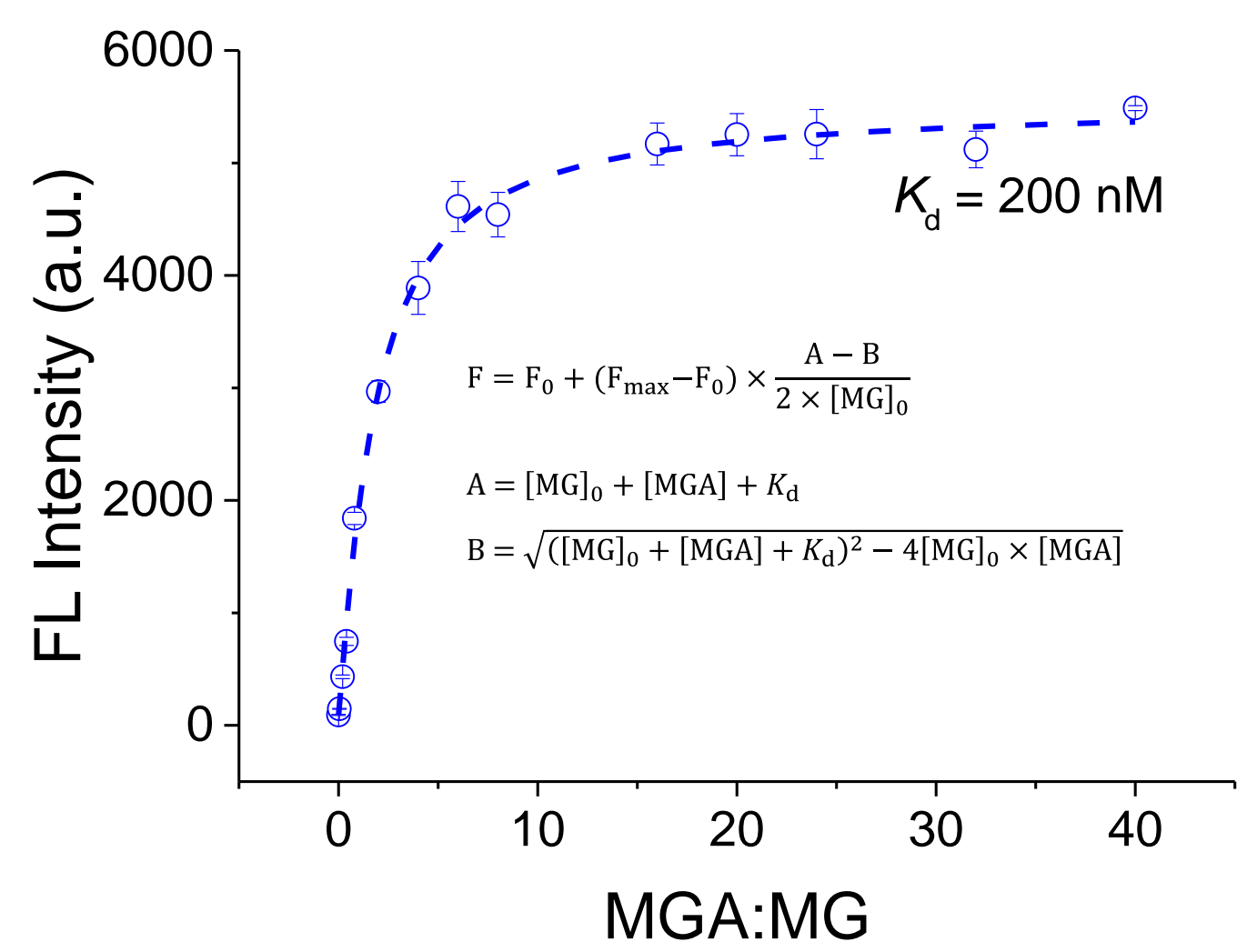

Figure 3.4 An illustration of $K_{\mathrm{d}}$ determination by fitting equation EQ (1) based on the fluorescence enhancement of MG $(150 \mathrm{nM})$ at ex/em $=620 \mathrm{~nm} / 656 \mathrm{~nm}$ upon binding to MGA.

\subsubsection{Fitting Equation for $K_{\mathrm{d}}$ Determination in Direct Binding}

The equation for $K_{\mathrm{d}}$ determination of MGA/MG binding has been deduced fundamentally, which has been reported [10-12] with some modifications:

$\mathbf{F}=\mathbf{F}_{\mathbf{0}}+\left(\mathbf{F}_{\max }-\mathbf{F}_{\mathbf{0}}\right) \times \frac{\left([\mathrm{MG}]_{0}+[\mathrm{MGA}]+K_{\mathrm{d}}\right)-\sqrt{\left([\mathrm{MG}]_{0}+[\mathrm{MGA}]+K_{\mathrm{d}}\right)^{2}-4[\mathrm{MG}]_{0} \times[\mathrm{MGA}]}}{2 \cdot[\mathrm{MG}]_{0}} \quad \mathbf{E Q}$

$\mathrm{F}$ and $\mathrm{F}_{0}$ are the fluorescence intensity in the presence and absence of aptamer, respectively. $F_{\max }$ is the fluorescence intensity while $[\mathrm{MGA}] \gg[\mathrm{MG}]_{0}$. $[\mathrm{MG}]_{0}$ and [MGA] are the concentrations of MG and MGA added into the binding system, and the $K_{\mathrm{d}}$ is the dissociation constant of the binding.

Process of deduction: 
Set the fraction of binding as

$\mathrm{f}=\frac{\mathrm{F}-\mathrm{F}_{0}}{\mathrm{~F}_{\max }-\mathrm{F}_{0}} \quad 0 \leq \mathrm{f} \leq 1$

and

$[\mathrm{MGA}]^{\prime}$ : concentration of free MGA in solution.

[MGA·MG]: concentration of MGA $\cdot \mathrm{MG}$ complex in solution.

[MG]: concentration of free MG in solution.

Thus,

$\mathrm{MGA} \cdot \mathrm{MG} \underset{K_{\mathrm{d}}}{\Leftrightarrow} \mathrm{MGA}+\mathrm{MG}$

$[\mathrm{MGA}]^{\prime}=K_{\mathrm{d}} \times[\mathrm{MGA} \cdot \mathrm{MG}] /[\mathrm{MG}]$

EQ (4)

$[\mathrm{MGA} \cdot \mathrm{MG}]=[\mathrm{MG}]_{0} \times \mathrm{f}$

EQ (5)

$[\mathrm{MG}]=[\mathrm{MG}]_{0} \times(1-\mathrm{f})$

EQ (6)

$[\mathrm{MGA}]=[\mathrm{MGA}]^{\prime}+[\mathrm{MGA} \cdot \mathrm{MG}]$

Based on EQ (7) and EQ (4),

$[\mathrm{MGA}]=K_{\mathrm{d}} \times[\mathrm{MGA} \cdot \mathrm{MG}] /[\mathrm{MG}]+[\mathrm{MGA} \cdot \mathrm{MG}]$

Based on EQ (5), EQ (6) and EQ (8),

$[\mathrm{MGA}]=K_{\mathrm{d}} \times \frac{\mathrm{f}}{1-\mathrm{f}}+[\mathrm{MG}]_{0} \times \mathrm{f}$

From EQ (9),

$[\mathrm{MG}]_{0} \times \mathrm{f}^{2}-\left([\mathrm{MG}]_{0}+[\mathrm{MGA}]+\mathrm{K}_{\mathrm{D}}\right) \times \mathrm{f}+[\mathrm{MGA}]=0$

Solve the mathematical equation EQ (10), and get

$\mathrm{f}=\frac{\left([\mathrm{MG}]_{0}+[\mathrm{MGA}]+K_{\mathrm{d}}\right) \pm \sqrt{\left([\mathrm{MG}]_{0}+[\mathrm{MGA}]+K_{\mathrm{d}}\right)^{2}-4[\mathrm{MG}]_{0} \times[\mathrm{MGA}]}}{2 \cdot[\mathrm{MG}]}$

And, $0 \leq \mathrm{f} \leq 1$, so

$\mathrm{f}=\frac{\left([\mathrm{MG}]_{0}+[\mathrm{MGA}]+K_{\mathrm{d}}\right)-\sqrt{\left([\mathrm{MG}]_{0}+[\mathrm{MGA}]+K_{\mathrm{d}}\right)^{2}-4[\mathrm{MG}]_{0} \times[\mathrm{MGA}]}}{2 \cdot[\mathrm{MG}]_{0}}$

Combine EQ (2) and EQ (12), and an equation for fitting of binding curve is obtained:

$\mathbf{F}=\mathbf{F}_{\mathbf{0}}+\left(\mathrm{F}_{\max }-\mathbf{F}_{\mathbf{0}}\right) \times \frac{\left([\mathrm{MG}]_{0}+[\mathrm{MGA}]+K_{\mathrm{d}}\right)-\sqrt{\left([\mathrm{MG}]_{0}+[\mathrm{MGA}]+K_{\mathrm{d}}\right)^{2}-4[\mathrm{MG}]_{0} \times[\mathrm{MGA}]}}{2 \cdot[\mathrm{MG}]_{0}} \quad \mathbf{E Q}$ (1) 
All the fitting calculations are performed by OriginPro 9.1. Fitting of the binding data in Figure 3.4 by equation EQ (1) results in a high $R^{2}(>0.99)$ and the $K_{\mathrm{d}}$ obtained compares well to the reported value.

Practically, there are two common methods for determination of $K_{\mathrm{d}}$ in direct binding assay. Besides the equation described above, another simple equation (Michaelis-Menten equation) is also applied to fit the binding data:

$\mathrm{F}=\frac{\left[\mathrm{F}_{\max }\right] \times[\mathrm{MGA}]}{K_{\mathrm{d}}+[\mathrm{MGA}]}$

However, equation is applicable while $[\mathrm{MG}]_{0}$ is well below the $K_{\mathrm{d}}$ in experimental setup (Under this situation, $[\mathrm{MGA}] \approx[\mathrm{MGA}]^{\prime}$ ) [13]. Even through a high $R^{2}(>0.99)$ and related similar $K_{\mathrm{d}}$ can also be obtained using EQ (13) for fitting, as shown in Figure 3.5, EQ (1) is preferred for this thesis.

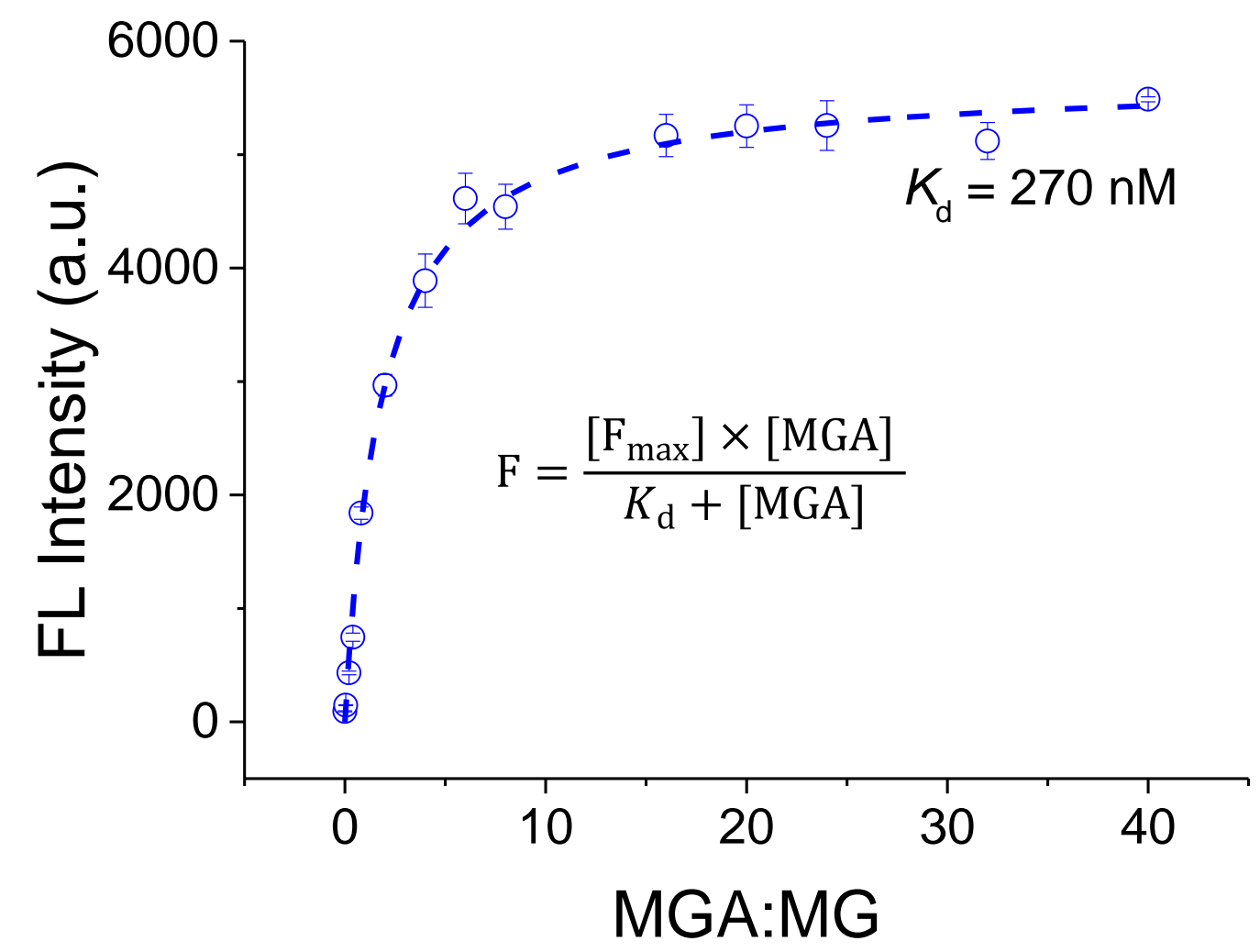

Figure 3.5 An illustration of $K_{\mathrm{d}}$ determination by fitting equation EQ (13) based on the fluorescence enhancement of $\mathrm{MG}(150 \mathrm{nM})$ at ex/em $=620 \mathrm{~nm} / 656 \mathrm{~nm}$ upon binding to MGA. 


\subsubsection{Determination of $K_{d}$ in Competitive Binding}

In some cases, MG derivatives or MG-stilbene conjugates may not have fluorescence enhancement upon binding to MGA. Thus, based on the fluorescence enhancement of MG upon binding to MGA, competitive binding assay is employed. In competitive binding mode, binding affinity is determined by varying concentrations of $\mathrm{MG}$ derivatives or MG-stilbene with MGA/MG complex concentration held constant. If the MG derivatives or MG-stilbene conjugates binds to MGA with competitively high affinity compared to MGA/MG binding, the fluorescence intensity of MGA/MG complex will decrease. The $K_{\mathrm{d}}$ can be determined by Cheng-Prusoff equation (EQ (14)), which is widely employed in competitive binding assay [14]:

$K_{\mathrm{d}}=\frac{\mathrm{IC}_{50}}{1+\frac{[\mathrm{MG}]}{K_{\mathrm{d}(\mathrm{MG})}}}$

$K_{\mathrm{d}}$ : dissociation constant of the conjugate

$\mathrm{IC}_{50}$ : concentration of the conjugate at $50 \%$ inhibition of MGA/MG complex

[MG]: concentration of MG

$K_{\mathrm{d}(\mathrm{MG})}$ : dissociation constant of $\mathrm{MG}$

Figure 3.6 shows an example of competitive binding of an MG-stilbene conjugate (MG$\mathrm{CH}_{2}-\mathrm{NH}$-stilbene-OMe (2) (Figure 3.1)) to MGA/MG complex. 


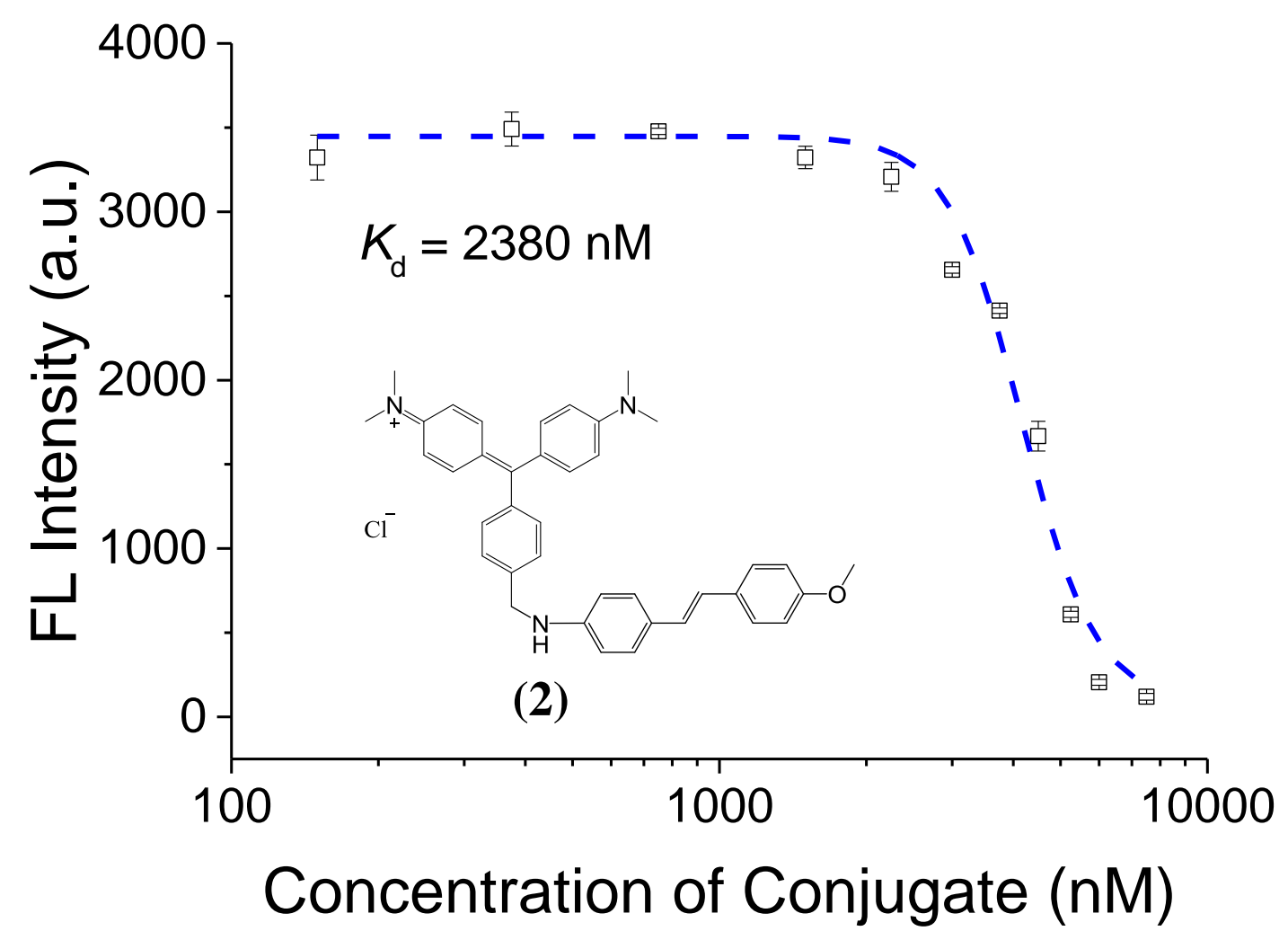

Figure 3.6 An illustration of $K_{\mathrm{d}}$ determination by competitive binding of a MG-stilbene conjugate (2) to MGA/MG complex, ex/em $=620 \mathrm{~nm} / 656 \mathrm{~nm}$.

\subsection{Fluorescence Emission and Fluorescence Decay Measurements of Stilbene Compounds by Spectrofluorophotometer}

Hypothesis of this thesis (section 1.3) focuses on the effect of aptamer binding on fluorescence decay of stilbene. Fluorescence decay property of stilbene molecules is another critical element in our designs while detectable fluorescence emission/fluorescence intensity is needed for fluorescence decay measurement. The basic principle of fluorescence emission has been described above (section 3.3.1). Fluorescence decay in emission maximum of stilbene molecules is due to the trans-cis isomerization of them upon UV irradiation. Like stilbene compounds, there are also other similar photoswichable molecules undergo isomerization. Azobenzene compounds (with -N=N-) undergo isomerization with a higher rate compared to stilbene compounds [15], which can be applied while faster signal reporting is needed. The time for trans-to-cis 
isomerization of azobenzenes is in picosecond or even femtosecond scale, while the time for stilbenes is mainly in picosecond scale [16-19]. Even limited by quantum yield, azobenzenes are still possible candidates for stilbenes in rapid sensing as methods for azobenzene fluorescence quantum yield enhancement have been developed [20,21], though the enhanced quantum yield is still small compared to that of stilbenes. $\mathrm{N}$ benzylideneaniline compounds (with $-\mathrm{C}=\mathrm{N}-$ ) with advantages of lower activation energy and convenient synthesis also have property of isomerization upon UV excitation [22]. However, they are rarely used for photoswitch due to the easy hydrolysis of imines [23]. Stilbene compounds are chosen in our design because of their appropriate detectable fluorescence decay time. However, if our hypothesis is supported by experimental data, when faster detection or monitoring is needed, azobenzene compounds should be good candidates, for instance, for detection in a microplate reader with pulse excitation.

In this study, fluorescence emission and fluorescence decay spectra are recorded by Shimadzu RF-5301PC spectrofluorophotometer (Japan). RF-5301PC provides continuous excitation which is suitable for fluorescence decay experiments of stilbene compounds, but M200 microplate reader can only supply pulse excitation. Briefly, for fluorescence emission spectra recording, appropriate concentration of stilbene sample is place in a quartz cuvette and the fluorescence emission spectrum is recorded by the Spectrofluorophotometer. Wavelength of emission maximum $\left(\lambda_{\max }\right)$ will be obtained by fluorescence emission spectrum scan, which will be used as emission wavelength in subsequent fluorescence decay measurement. Excitation/emission wavelengths, testing solvent systems and concentrations vary according to certain stilbene compounds based on their specific fluorescent properties. Figure 3.7 displays the fluorescence emission spectrum of a stilbene molecule 4,4'-Diisothiocyanatostilbene-2,2'-disulfonic acid disodium salt (DIDS) (3) (Figure 3.1). For fluorescence decay measurement, appropriate concentration of stilbene sample (varies in different kinds of stilbene compounds) is place in a quartz cuvette, and the fluorescence intensity at $\lambda_{\max }$ is recorded as a function of time, and the apparent decay rate is analyzed by a first order fitting which will be introduced below. 


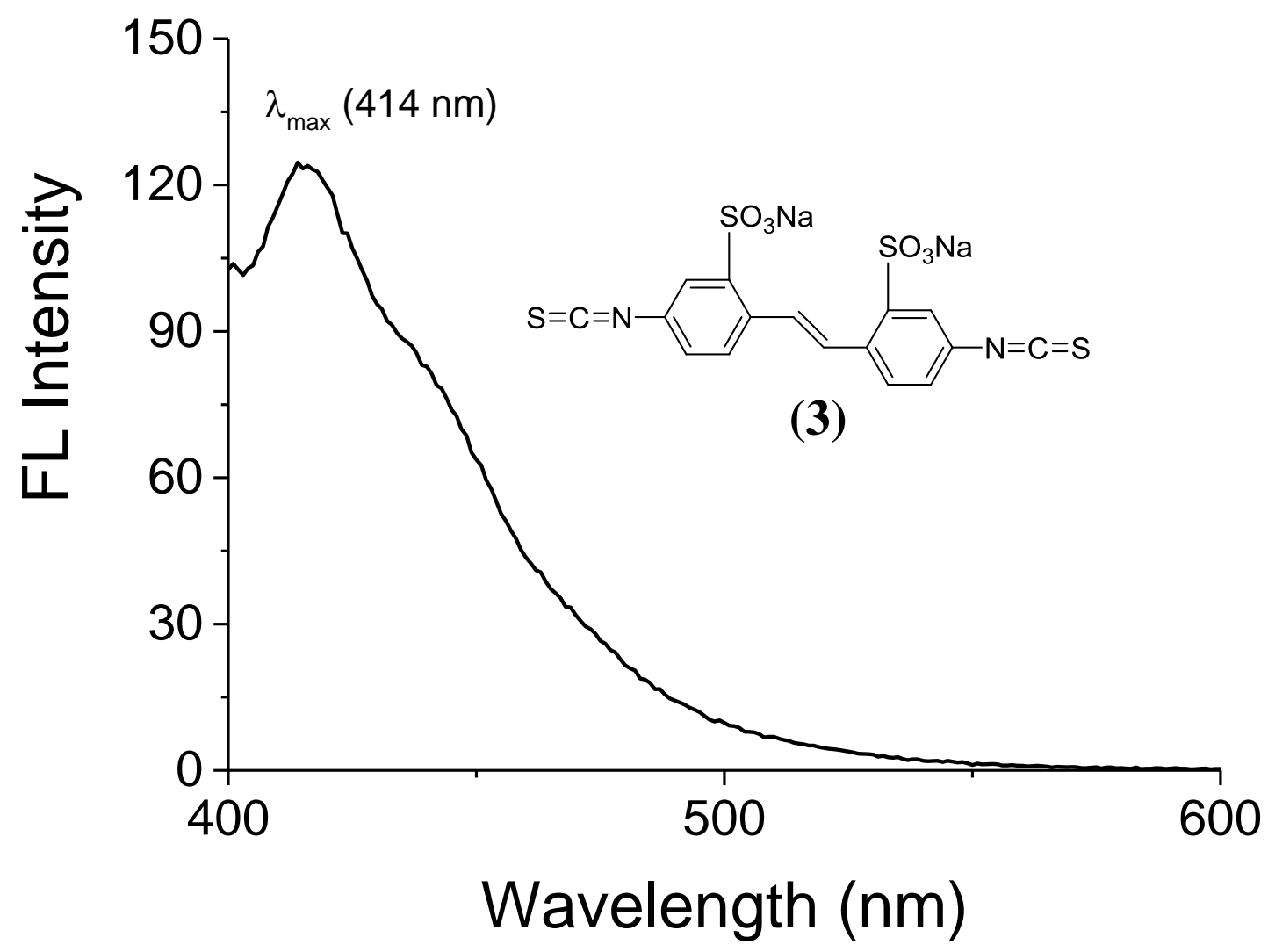

Figure 3.7 Fluorescence emission spectrum of stilbene compound DIDS (3) with $\lambda_{\mathrm{ex}}=340$ nm.

Analysis of apparent fluorescence decay rate of stilbene molecules is adapted from literature [24]. Calculation of apparent fluorescence decay rate follows the equation $\mathrm{dI}_{\mathrm{t}} / \mathrm{dt}$ $=-k_{\text {app }} \times I_{t}+$ constant, which can be rearranged into a first order equation for convenient fitting analysis:

$\mathrm{I}_{\mathrm{t}}=\mathrm{A} \times \mathrm{e}^{-k_{\mathrm{app}} \times \mathrm{t}}+\mathrm{B}$

t: decay time.

$k_{\text {app: }}$ apparent fluorescence decay rate constant (first order).

$\mathrm{I}_{\mathrm{t}}$ : fluorescence intensity at time $\mathrm{t}$.

A: total change of fluorescence intensity.

B: final fluorescence intensity.

Figure 3.8 shows the fluorescence decay spectrum and apparent fluorescence decay rate constant of DIDS (3). All the fitting calculations are performed by OriginPro 9.1. 


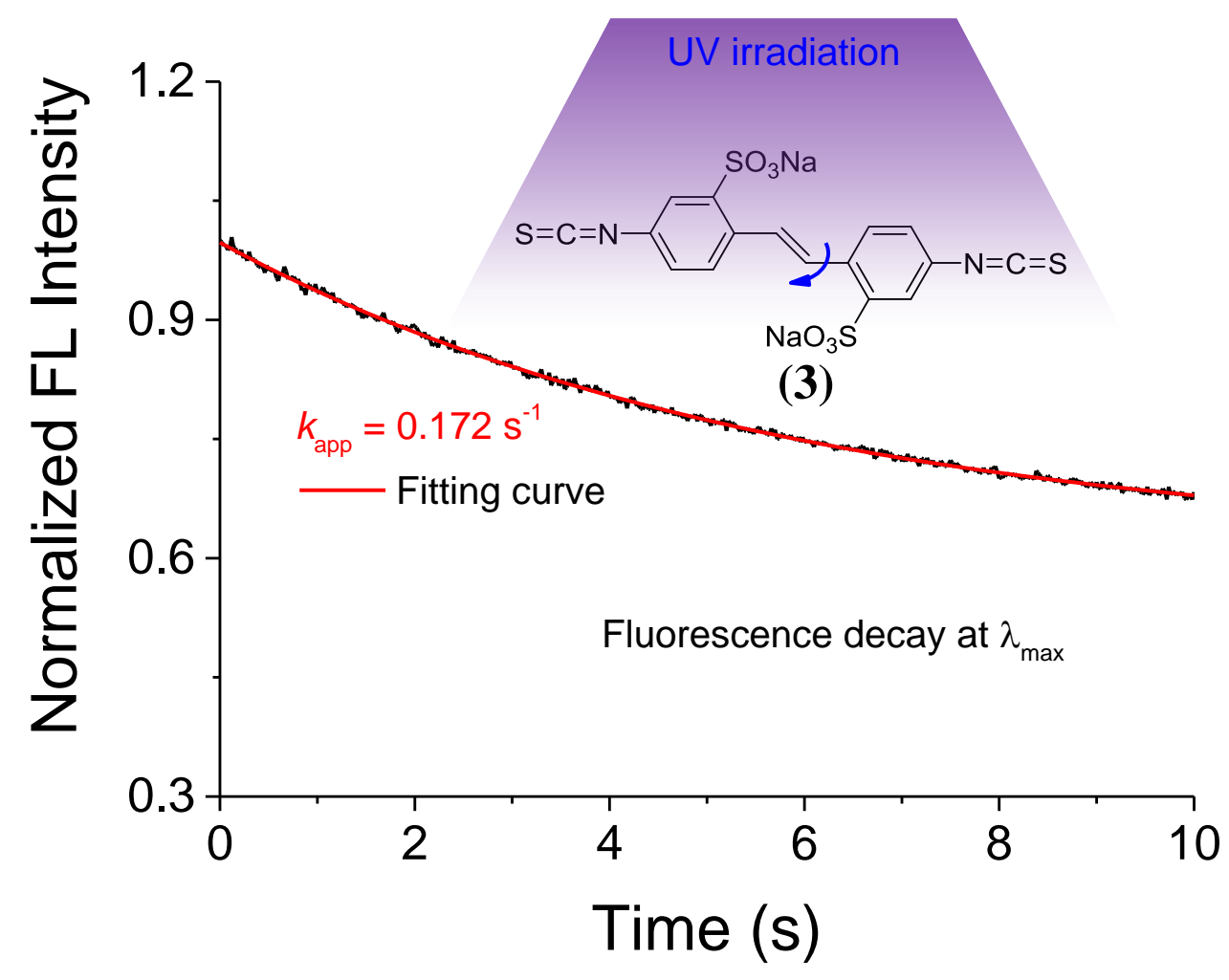

Figure 3.8 Fluorescence decay spectrum at wavelength of emission maximum $\left(\lambda_{\max }\right)$ and the corresponding apparent fluorescence decay rate constant $k_{\text {app }}$ (calculated by EQ (15)) of stilbene compound DIDS (3) $(e x / e m=340 \mathrm{~nm} / 414 \mathrm{~nm})$.

\subsection{Preparation of MGA-SITS Conjugate (5) (Figure 3.1)}

\subsubsection{Synthesis and Purification of MGA-SITS Conjugate}

There are several usual approaches used for oligonucleotide/protein bioconjugation with ligand: amine-isothiocyanate reaction, azide-alkyne click reaction, thiol-maleimide, amino-N-hydroxysuccinimide ester [25]. Selection of reaction approaches mainly depends on the active groups and difficulty in obtaining of aptamer or stilbene compounds. In this study, commercially available 4-Acetamido-4'-isothiocyanato-2,2'stilbenedisulfonic acid disodium salt (SITS) (4) (Figure 3.1) with one active group (isothiocyanate) is paired with amine modified MGA $\left(\mathrm{MGA}-\mathrm{NH}_{2}\right)$, purchased from AITbiotech Pte Ltd Singapore. The synthesis of MGA-SITS follows a typical amineisothiocyanate reaction protocol (Figure 3.9). In our study, the reaction is conducted 
based on previous methods [26]. Briefly, $\mathrm{MGA}-\mathrm{NH}_{2}$ reacts with $100 \mathrm{X}$ excess of SITS in $\mathrm{pH}=9$ sodium bicarbonate reaction buffer for 12 hours at room temperature.

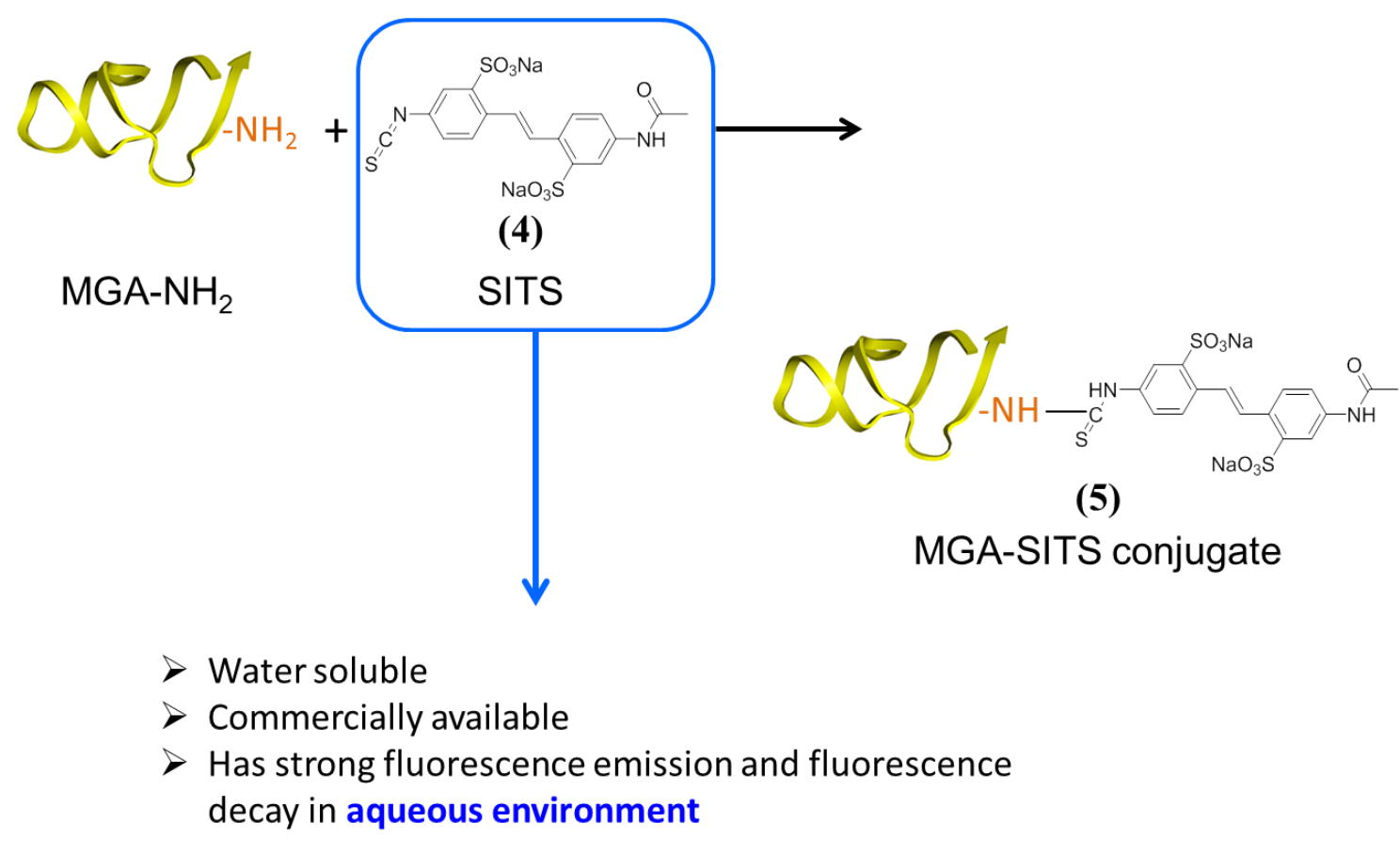

Figure 3.9 Synthesis of MGA-SITS conjugate (5).

Purification of conjugate and removal of excess SITS is done by ultrafiltration with Amicon Centrifugal Filter based $10 \mathrm{k} \mathrm{Da}$ membrane. The reaction mixture was centrifuged, concentrated, and rediluted with binding buffer to be centrifuged again. The cycle was repeated 9 times to yield a SITS-free MGA-SITS. A product yield of $>80 \%$ can be obtained. Nanodrop UV-Vis spectrometer $(260 \mathrm{~nm})$ and HPLC-DAD are used to monitor the purification. A simplified schematic description of HPLC-DAD on the typical working flow/principle has been drawn in Figure 3.10. $\mathrm{C}_{18}$ reversed-phase chromatography is able to separate the MGA- $\mathrm{NH}_{2}$ and SITS compounds for analytical purpose. Additionally, DAD detector can record a full spectrum of the peaks in a specific retention time. However, DAD has shortcomings such as relatively low sensitivity and large noise compared to UV-Vis HPLC detector, but this is not an issue in our analysis as our analytes are in relatively high concentration. This HPLC-DAD system can give information on how much free (not conjugated) SITS in the purified product and thus ensure the purity of the final product. Figure 3.11 gives an example of our $\mathrm{C}_{18}$ reversed- 
phase HPLC-DAD chromatography on analysis of MGA- $\mathrm{NH}_{2}$ with mobile phase of ammonium acetate buffer/acetonitrile, where the spectrum of $\mathrm{MGA}-\mathrm{NH}_{2}$ and the full spectra over the elution period are also displayed.

\subsubsection{Characterization of MGA-SITS Conjugate}

HPLC-DAD and MALDI-TOF mass spectrometry are used for the characterization of the synthesized conjugate. HPLC-DAD is used for monitoring the change in UV-Vis spectrum of the MGA-NH 2 peak (Absorbance at $260 \mathrm{~nm}$ ). If the SITS is successfully grafted on MGA, absorbance at $340 \mathrm{~nm}$ should increase at the peak retention time of MGA-NH $\mathrm{N}_{2}$, indicating the successfully grafting of SITS on MGA. The procedure of HPLC-DAD measurement is the same as that described in section 3.5.1.

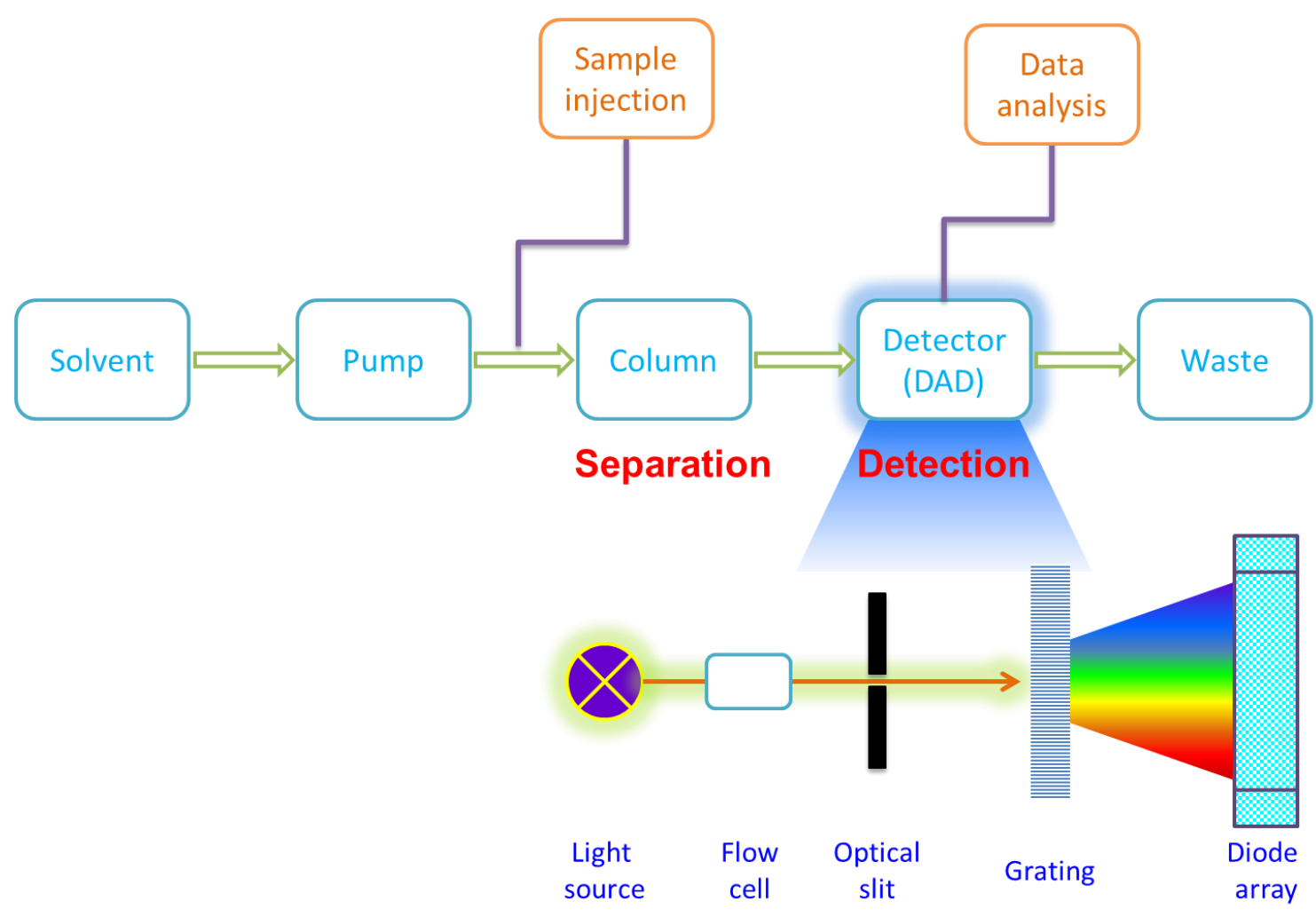

Figure 3.10 Schematic simplified working flow/principle of HPLC and DAD. 


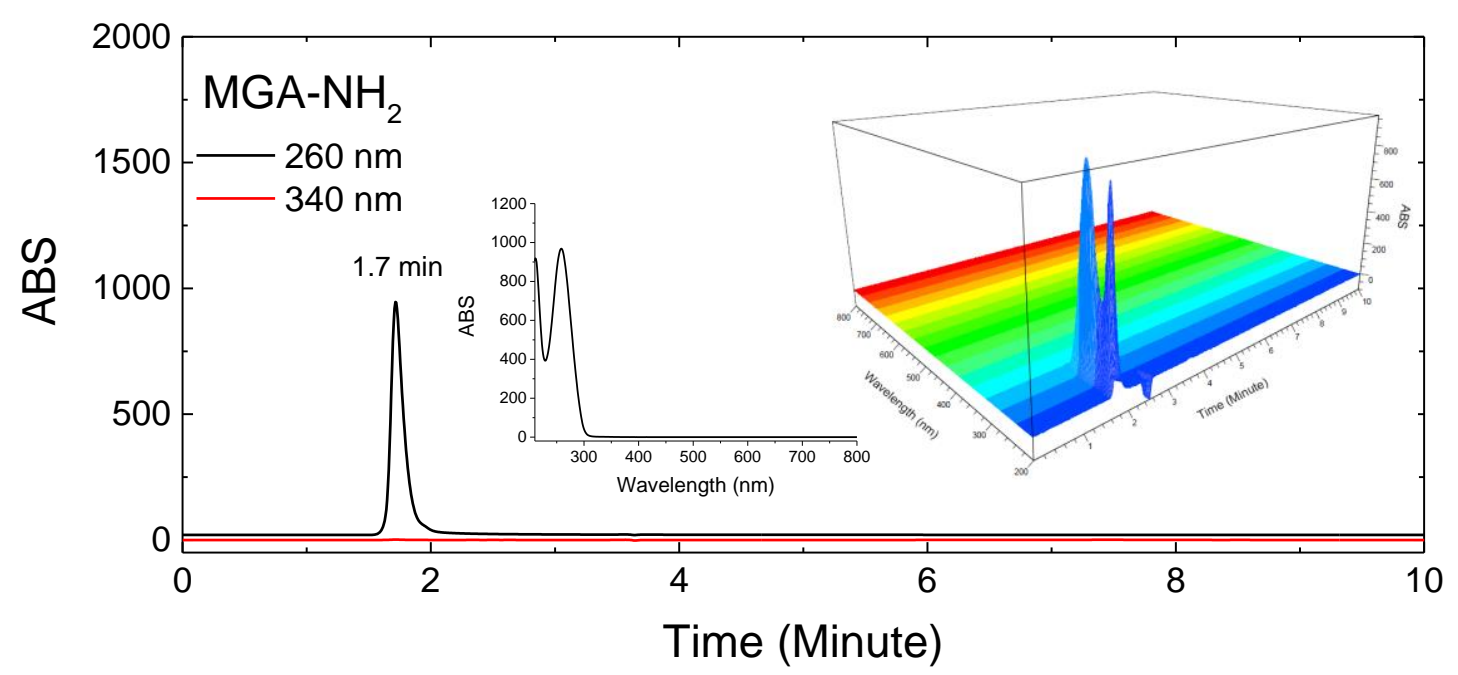

Figure 3.11 $\mathrm{C}_{18}$ reversed-phase HPLC-DAD chromatography of MGA- $\mathrm{NH}_{2}$.

MALDI-TOF mass spectrometry is a technique for measurement of molecular weight of targeted compounds based on the time of flight of the charged compound. The working principle of MALDI-TOF mass spectrometry is shown in Figure 3.12 [27]: Crystals form upon the mixing of analyte and saturated matrix; then ionization takes place after laser irradiation (typical wavelength is $337 \mathrm{~nm}$ ); the formed sample ions will be accelerated by a high voltage (from $15 \mathrm{kV}$ to $25 \mathrm{kV}$ ) within the electrostatic field; then the molecules in sample will be separated in the time of flight tube according to the time of flight, which depends on the mass-to-charge ratio $(\mathrm{m} / \mathrm{z})$ of molecules in defined experimental conditions: $\left(\mathrm{mv}^{2}\right) / 2=$ zeEs — while experimental conditions e, E and s are fixed, velocity $\mathrm{v}$ decrease as mass-to-charge ratio $\mathrm{m} / \mathrm{z}$ increase, resulting in an increase of time of flight to reach the detector; finally, sample ions will reach the detector and corresponding spectrum will be record. Values of $\mathrm{m} / \mathrm{z}$ which is indicated by flight of time can be directly reflected in MALDI-TOF mass spectrum.

The relatively small error (less than 0.5\%) and high sensitivity of MALDI-TOF mass spectrometry system enables it to tell the difference of $\mathrm{MGA}-\mathrm{NH}_{2}$ before and after conjugation with SITS, which is sufficient for characterization of the conjugate. 2', 4', 6'-Trihydroxyacetophenone monohydrate (THAP) and 3-Hydroxypicolinic acid (3-HPA) are excellent matrices for oligonucleotide in MALDI-TOF mass spectrometry, which are able to promote the ionization of oligonucleotide and reduce fragmentation [28]. THAP 
matrix is mixed with matrix: MGA-SITS $=1: 1(\mathrm{v} / \mathrm{v})$. Prior to the test, MGA-SITS undergoes desalting pretreatment by Amicon Centrifugal Filter with $100 \mathrm{mM}$ ammonium citrate $\left(\mathrm{pH}=6\right.$ ), which removes the interference from metal ions (i.e. $\mathrm{Na}^{+}$and $\mathrm{Mg}^{2+}$ ). Results display that the mass spectrum obtained is sufficient to suggest the conjugation of SITS on MGA (Figure 3.13).

Size exclusion chromatography (SEC) is also an approach for characterization of the conjugation reaction based on column retention time. However, the resolution of most SEC column is inadequate to separate the molecular weight difference of a SITS molecule ( $\sim 500 \mathrm{Da})$ based on the retention time or with static light. Moreover, SEC method requires large sample amount $(10-100 \mu \mathrm{g})$ for injection. Thus, SEC method is not an ideal technique under our experimental conditions. NMR is another method directly showing the structure of RNA. Compared to the methods above, NMR may even give the information on trans-cis isomerization of SITS grafted on MGA. However, NMR experiments require a large amount of costly oligonucleotide, and the signal of SITS molecule may also be interfered by oligonucleotide signals. Thus, MALDI-TOF mass spectrometry should be an appropriate method for the characterization of the MGA-SITS conjugate, while HPLC can also give confirmation on the successfully conjugation of stilbene to aptamer. 


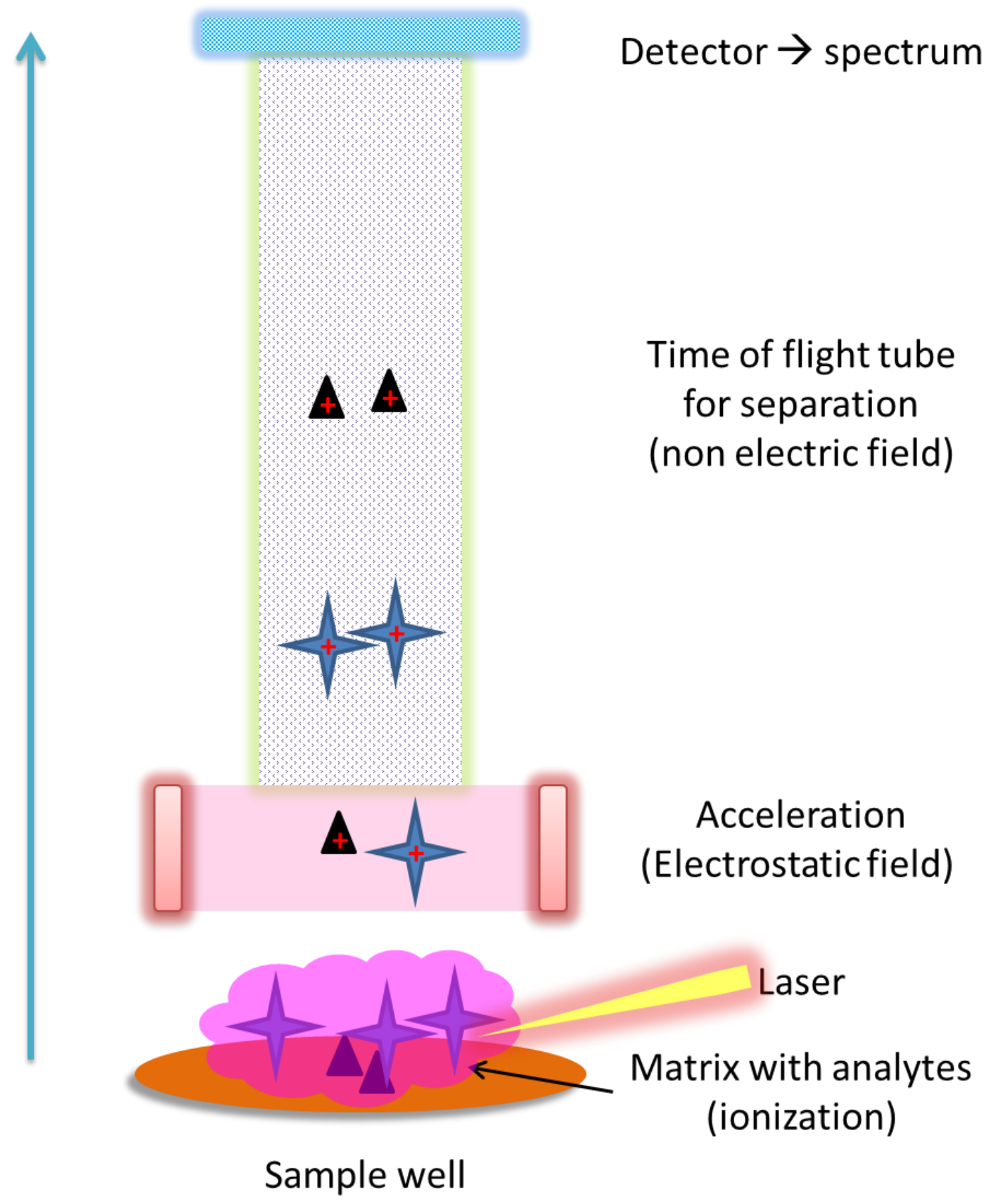

Figure 3.12 Schematic principle of MALDI-TOF mass spectrometry. 


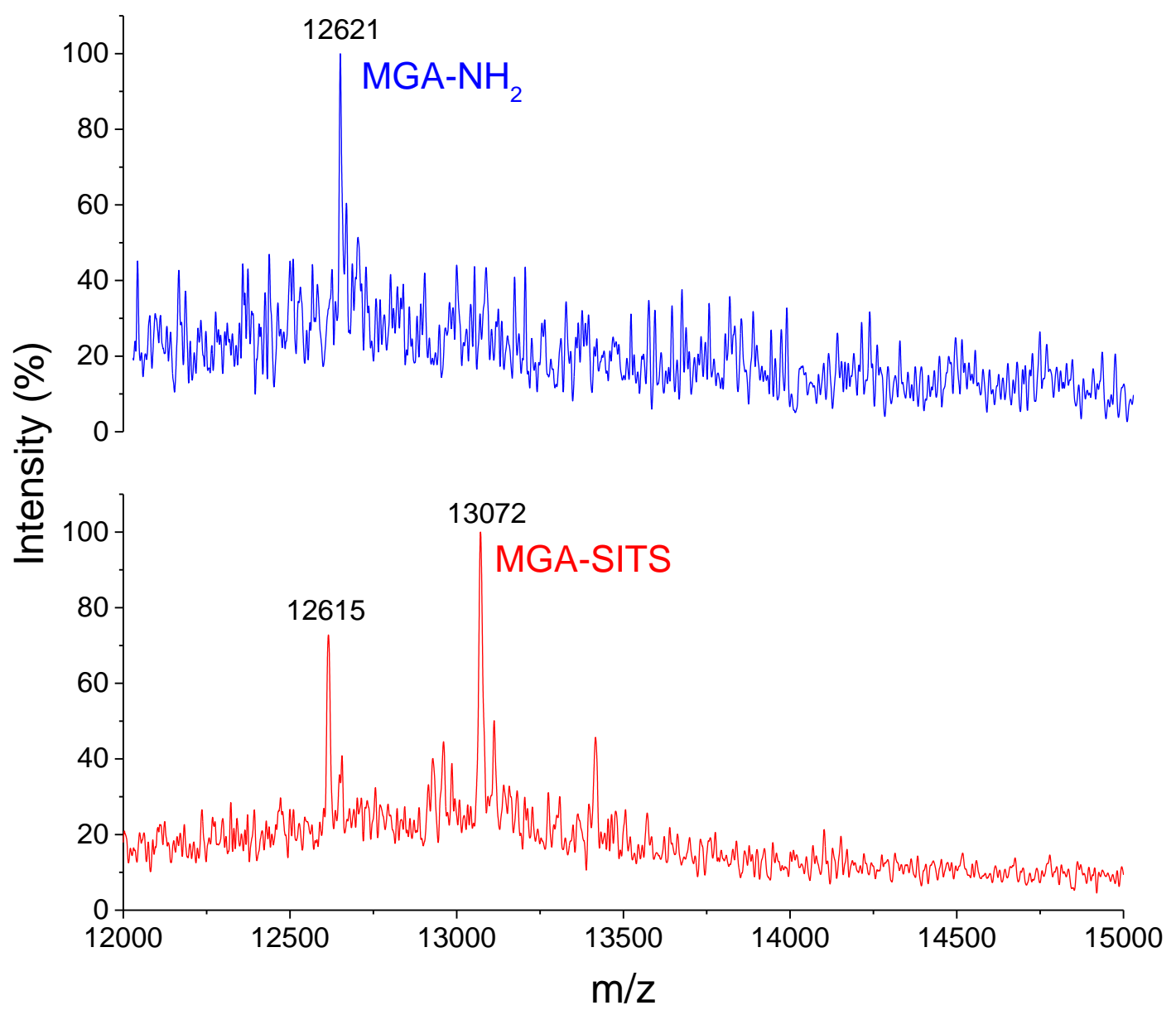

Figure 3.13 MALDI-TOF spectra of $\mathrm{MGA}-\mathrm{NH}_{2}$ and MGA-SITS conjugate (5). Top (blue): MGA- $\mathrm{NH}_{2}$ before conjugation. Bottom (red): SITS grafted MGA-NH $\mathrm{NHG}_{2}$ (MITS conjugate (5)). The $\mathrm{m} / \mathrm{z}$ increases for 457 units after the conjugation, indicating the molecular weight of the aptamer molecule increases $457 \mathrm{Da}$ upon attachment of the SITS fragment. The MALDI-TOF results indicate the SITS is successfully conjugated on MGA.

\subsection{Kinetic Study on MG Conversion}

Kinetic study on MG or malachite green carbinol base (MGOH) (6) (Figure 3.1) conversion is accomplished by monitoring UV-Vis absorbance of MG at absorbance maximum ( 620 nm). Briefly, MG or MGOH solution is placed into a Varian Cary 50 $\mathrm{UV}-\mathrm{Vis}$ spectrophotometer and the UV-Vis spectra are recorded every 5 min for 12 hours. According to Beer-Lambert law, concentration of analyte is directly proportional to the specific absorbance. The working principle of UV-Vis spectrophotometer is introduced in 
Figure 3.14. MG has a significant absorbance maximum at wavelength around $620 \mathrm{~nm}$ while MGOH has no significant absorbance at the region around $620 \mathrm{~nm}$. Studies by HPLC have indicated that MG can hydrolyze to MGOH under certain conditions. Thus, UV-Vis recording at wavelength of MG absorbance maximum is a rapid and sufficient method to monitor the hydrolysis of MG to MGOH, and vice versa. Figure 3.15 shows an example of kinetic study of MG conversion monitored by UV-Vis spectrophotometer.

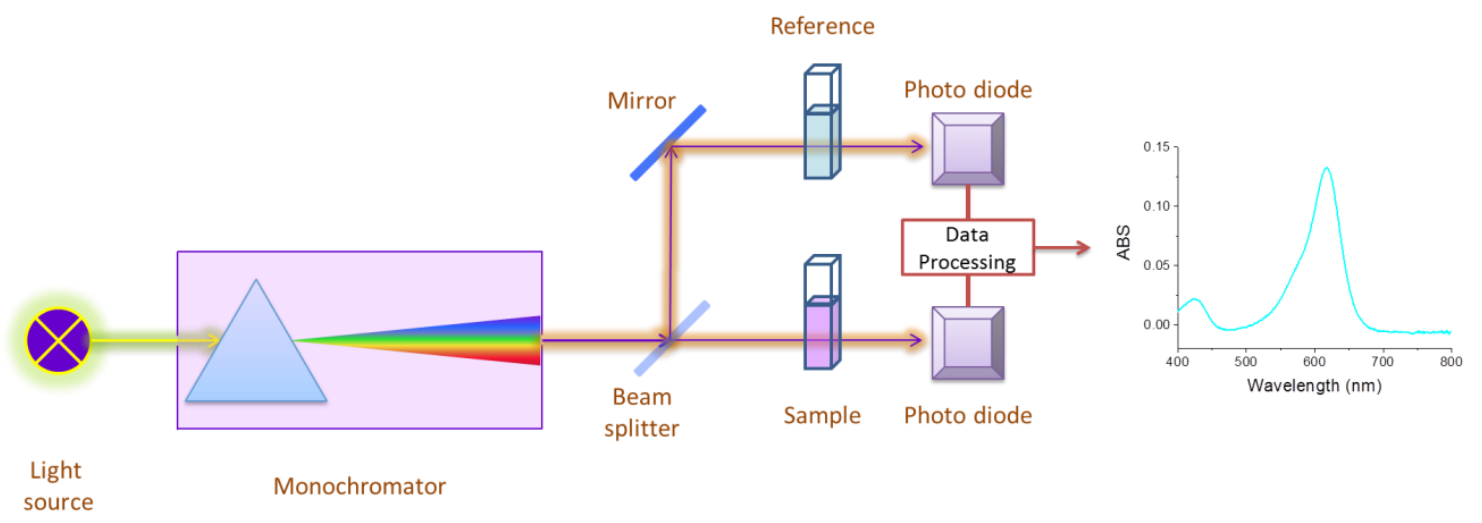

Figure 3.14 Schematic working principle of UV-Vis spectrophotometer. 


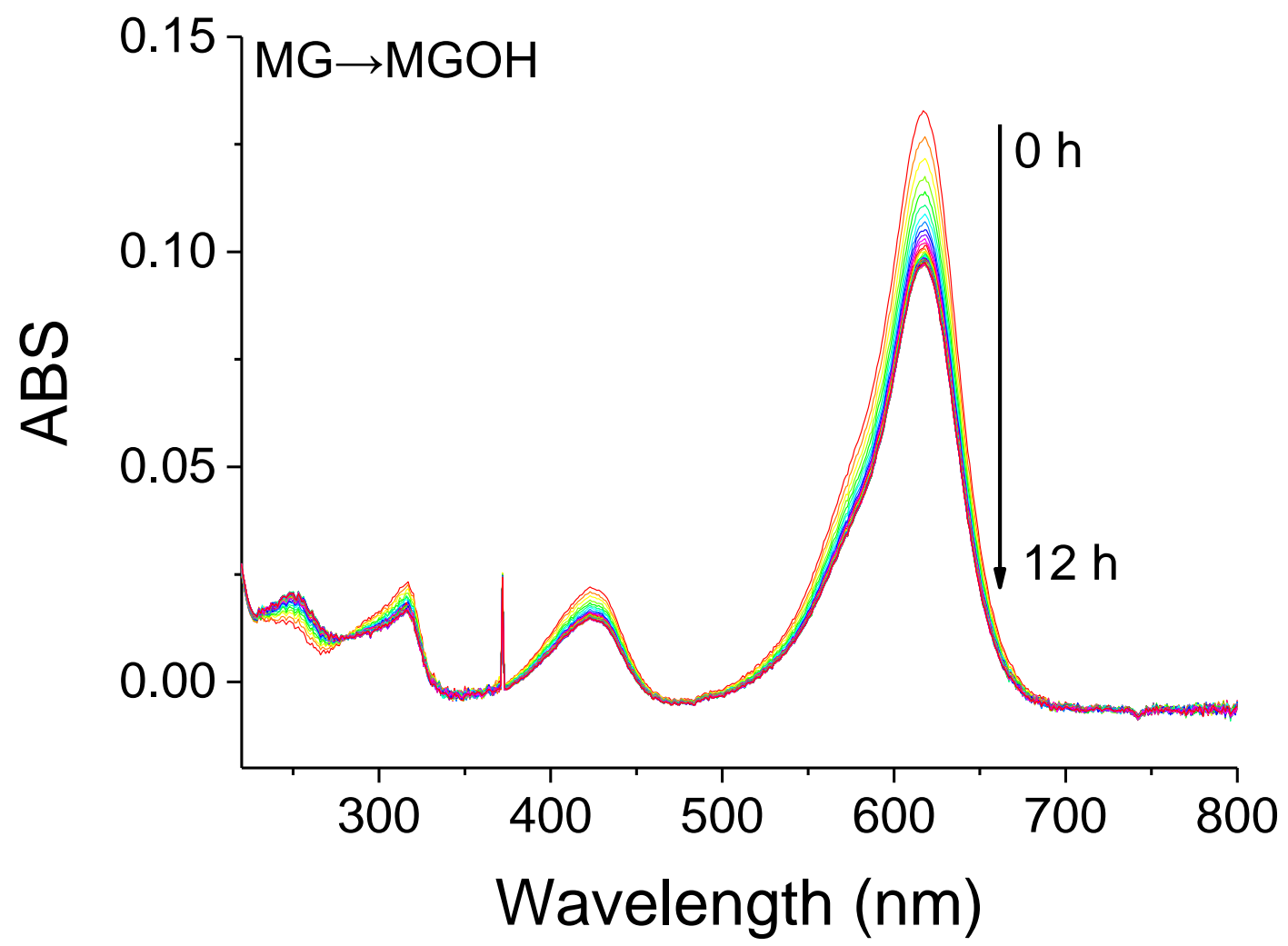

Figure 3.15 An example of kinetic study on MG conversion by UV-Vis spectrophotometer.

\subsection{Statistical Analysis}

All the statistics were performed by OriginPro 9.1 (OriginLab Corporation, Northampton, MA 01060 USA). Linear regressions $R^{2}$ were determined by ordinary least squares with a minimum of $n=3$ data points. To test the statistical difference between two groups of values, hypothesis testing was performed by Student's t-test with significance level $p<$ 0.05 (two-tailed test).

\section{References:}

[1] J. F. Lee; J. R. Hesselberth; L. A. Meyers; A. D. Ellington Nucleic Acids Research 2004, 32, D95.

[2] C. Baugh; D. Grate; C. Wilson Journal of Molecular Biology 2000, 301, 117. 
[3] J. Flinders; S. C. DeFina; D. M. Brackett; C. Baugh; C. Wilson; T. Dieckmann ChemBioChem 2004, 5, 62.

[4] W. C. Andersen; S. B. Turnipseed; C. M. Karbiwnyk; R. H. Lee; S. B. Clark; W. D. Rowe; M. R. Madson; K. E. Miller Analytica Chimica Acta 2009, 637, 279.

[5] M. Jing; M. T. Bowser Analytica Chimica Acta 2011, 686, 9.

[6] G. J. Parker; T. L. Law; F. J. Lenoch; R. E. Bolger Journal of Biomolecular Screening 2000, $5,77$.

[7] F. E. AHMED; J. E. WILEY; D. A. WEIDNER; C. BONNERUP; H. MOTA Cancer Genomics - Proteomics 2010, 7, 303.

[8] J. R. Babendure; S. R. Adams; R. Y. Tsien Journal of the American Chemical Society 2003, 125, 14716 .

[9] J. Bernard Da Costa; T. Dieckmann Molecular BioSystems 2011, 7, 2156.

[10] Y. Wang; R. R. Rando Chemistry \& Biology 1995, 2, 281.

[11] Y. Wang; J. Killian; K. Hamasaki; R. R. Rando Biochemistry 1996, 35, 12338.

[12] K. Hamasaki; J. Killian; J. Cho; R. R. Rando Biochemistry 1998, 37, 656.

[13] T. D. Pollard Molecular Biology of the Cell 2010, 21, 4061.

[14] C. Yung-Chi; W. H. Prusoff Biochemical Pharmacology 1973, 22, 3099.

[15] P.-C. Yang; C.-L. Lee; W.-C. Chen; C.-Y. Lin The Canadian Journal of Chemical Engineering 2013, 91, 1513.

[16] S. A. Kovalenko; A. L. Dobryakov Chemical Physics Letters 2013, 570, 56.

[17] J. García-Amorós; D. Velasco Beilstein Journal of Organic Chemistry 2012, 8, 1003.

[18] A. A. Beharry; G. A. Woolley Chemical Society Reviews 2011, 40, 4422.

[19] C. Jiang; R. Xie; F. Li; R. E. Allen Chemical Physics Letters 2009, 474, 263.

[20] M. R. Han; Y. Hirayama; M. Hara Chemistry of Materials 2006, 18, 2784.

[21] M. Han; D. Ishikawa; E. Muto; M. Hara Journal of Luminescence 2009, 129, 1163.

[22] Y. Luo, Freie Universität Berlin, 2011.

[23] N. A. Alexander, University of Canterbury, 2006.

[24] V. Papper; G. I. Likhtenshtein; N. Medvedeva; D. V. Khoudyakov Journal of Photochemistry and Photobiology A: Chemistry 1999, 122, 79.

[25] Y. Shu; F. Pi; A. Sharma; M. Rajabi; F. Haque; D. Shu; M. Leggas; B. M. Evers; P. Guo Advanced Drug Delivery Reviews 2014, 66, 74. 
[26] G. T. Hermanson In Bioconjugate Techniques (Third edition); Academic Press: Boston, 2013, p 229.

[27] L. F. Marvin; M. A. Roberts; L. B. Fay Clinica Chimica Acta 2003, 337, 11.

[28] C. M. Castleberry; C.-W. Chou; P. A. Limbach In Current Protocols in Nucleic Acid Chemistry; John Wiley \& Sons, Inc.: 2008. 
Chapter 3 


\section{Chapter 4}

\section{Conditions for Malachite Green Aptamer Binding}

This chapter studies the binding conditions for malachite green aptamer $(M G A)$ binding with malachite green $(M G)$, and the stability of $M G A, M G$ and their binding, which pave the foundation for the hypothesis testing. Binding affinity and fluorescence intensity of $M G A / M G$ binding were found to decrease over time. It was hypothesized the hydrolysis of MG to malachite green carbinol base (MGOH) resulted in the loss of fluorescence, while our results suggest this conversion and confirm the integrity of MGA over 48 hours. Interestingly, we noted the employment of organic additives retained the fluorescence and binding over time, and synergistic effect from both organic additives and aptamer on stabilizing MG lability were found and discussed. Limited impact from organic additives on binding affinity was found. Regarding to the hydrophobicity of stilbene, organic additives are required in $M G A / M G$ binding. Additionally, effect of $p H$ on $M G A / M G$ binding was also investigated. The results obtained in this chapter establish optimal binding conditions for hypothesis testing and provide useful information on the future aptameric sensing.

*This section published substantially as: Y. Zhou; H. Chi; Y. Wu; R. S. Marks; T. W. J. Steele Talanta 2016, 160, 172-182. 


\subsection{Introduction}

Prior to the test of the hypothesis, basic experimental conditions need to be defined for reliable binding. Malachite green aptamer (MGA) with its ligand malachite green (MG) (1) is a model study in the thesis, while triphenylmethane compound MG may undergo hydrolysis and the RNA aptamer MGA may degrade. It was reported that MG can transfer to its derivatives like leucomalachite green and malachite green carbinol base $(\mathrm{MGOH})(6)$ in aqueous environment (Figure 4.1A) [1,2]. On the other hand, RNA is known to susceptible to degradation. Thus, it is essential to investigate the stability of MGA, MG and their binding, and establish suitable experimental conditions for the binding, which is the prerequisite for hypothesis testing. As reviewed in literature (section 2.2.8), MGA/MG binding results in an enhanced fluorescence quantum yield (more than 2000X) upon recognition, which can be employed for study the binding interaction (Figure 4.1B) [3]. The initial binding affinity (or equilibrium dissociation constant, $K_{\mathrm{d}}$ ) was determined to be comparable to literature at time 0. However, it was found that MGA/MG binding had increasing apparent $K_{\mathrm{d}}$ as determined through the first order rate loss of their fluorescence intensity over time, indicating variation in MGA, MG, or their binding. Electrophoresis analysis suggests MGA kept its integrity with 48 hours while HPLC results indicated MG converted to $\mathrm{MGOH}$ over time, and thus the hydrolysis of MG to MGOH was hypothesized to be the cause of fluorescence intensity loss (Figure 4.1A). Various organic additives (acetonitrile (ACN), dimethyl sulfoxide (DMSO) and ethanol $(\mathrm{EtOH}))$ were investigated to retain the binding stability [4]. Both fluorescence intensity and apparent $K_{\mathrm{d}}$ were less sensitive to time in the presence of the organic additives, and the protection became more apparent with increasing organic additive percentage (up to $10 \%, \mathrm{v} / \mathrm{v}$, the same to all description on organic additive percentage). Additionally, the presence of the organic additives only has limited impact on the 0 time $K_{\mathrm{d}}$. In fact, hydrophobic stilbene (miLog $\mathrm{P}=4.5$, calculated by Molinspiration Cheminformatics 2016: www.molinspiration.com) normally requires the involvement of organic additives due to its solubility issue, and the results enable the application of these additives on MGA/MG binding for the test of hypothesis. This simple strategy minimizes deviations in the MGA/MG binding analysis, and also indicates an approach for 
stabilizing the aptamer binding of other triphenylmethane, triarylmethane, or carbocationsusceptible ligands bindings susceptible to $\mathrm{S}_{\mathrm{N}} 1$ hydrolysis. Additionally, other studies like $\mathrm{pH}$ effect investigation are also conducted. Various results and conclusions obtained in this chapter establish optimal conditions for MGA/MG binding as well as hypothesis testing, and provide meaningful information and evidence for the future MGA based biosensing.

A

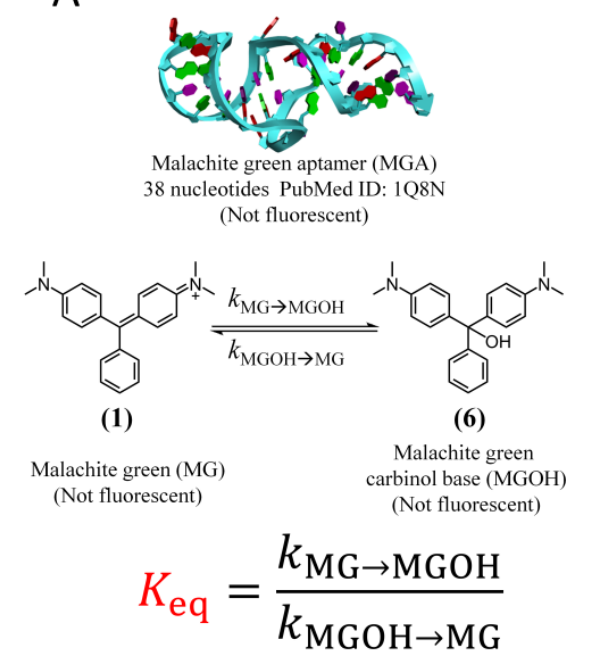

B

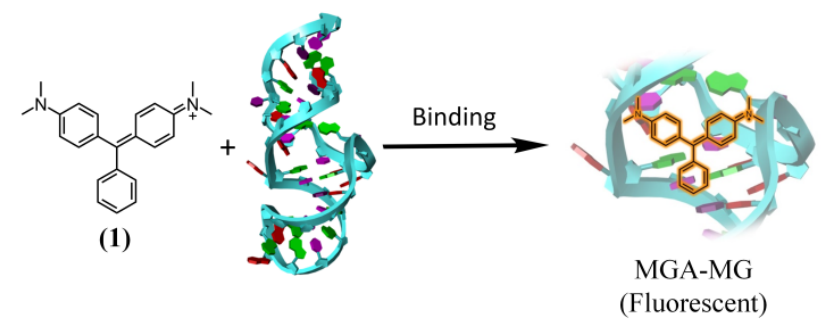

$K_{d}=\frac{[\mathrm{MGA}][\mathrm{MG}]}{[\mathrm{MGA}-\mathrm{MG}]}$

Figure 4.1 (A) Structure of malachite green aptamer (MGA) (6), malachite green (MG) (1) and malachite green carbinol base (MGOH). (B) Binding of MGA to MG, with fluorescence enhancement obtained. (Reprinted with permission from reference [4].)

\subsection{Materials and Methods}

\subsubsection{Materials}

Malachite green aptamer (MGA) RNA (5' GGAUCCCGACUGGCGAGAGCCAGGUAACGAAUGGAUCC-3') was purchased from AITbiotech Pte Ltd Singapore. Acetonitrile (ACN) (HPLC grade) was purchased from Tedia, USA. Dimethyl sulfoxide (DMSO) was purchased from Alfa Aesar, USA. Ethanol (EtOH) was purchased from Fisher Scientific, Singapore. Malachite green oxalate (MG) (1) and malachite green carbinol base $(\mathrm{MGOH})(6)$ were purchased from 
Sigma Aldrich, Singapore. Polystyrene microplates (96-well) were purchased from Corning, USA. All chemicals and materials were used as received.

Binding buffer ( $\mathrm{pH}=6.7$ unless otherwise specified) consisting of $5.65 \mathrm{mM} \mathrm{NaH}_{2} \mathrm{PO}_{4}$, $4.35 \mathrm{mM} \mathrm{Na} 2 \mathrm{HPO}_{4}, 10 \mathrm{mM} \mathrm{MgCl} 2$ and $1 \mathrm{mM} \mathrm{NaCl}$ was prepared according to the literature $[5,6]$.

\subsubsection{MGA/MG Binding Assay}

The MGA/MG binding was determined by monitoring the fluorescence enhancement of MG upon binding according to literature [3], and the method has been described in section 3.3.1 and section 3.3.2. In details, $K_{\mathrm{d}}$ was determined varying ratios 0:1 to 40:1 MGA:MG with MG concentration held constant (150 $\mathrm{nM})$ in binding buffer or organic additive/binding buffer mixture. Fluorescence of MG was immediately assayed from $0 \mathrm{~h}$ to $24 \mathrm{~h}$ in 96-well polystyrene plates with a Tecan Microplate spectrofluorometer (Infinite M200, Tecan Asia PTE LTD, Singapore), at ex/em = $620 \mathrm{~nm} / 656 \mathrm{~nm}$.

Fitting of binding equilibrium dissociation constant $K_{\mathrm{d}}$ was achieved by using function EQ (1) in section 3.3.2, where the rationale of such fitting is also described. Non-linear fitting and statistical analysis were performed by OriginPro 9.1.

In the investigation of organic additives on fluorescence of MGA/MG complex post decay, DMSO was added into the complex $5 \mathrm{~h}$ after the binding experiment started.

\subsubsection{High Performance Liquid Chromatography (HPLC)-Diode Array Detector (DAD) Study on MG Hydrolysis}

An Agilent 1200 series HPLC system with DAD was used to investigate the stability of MG. Chromatographic separation was conducted using a $5 \mu \mathrm{m} \times 4.6 \mathrm{~mm} \times 250 \mathrm{~mm}$ Ultisil $^{\mathrm{TM}} \mathrm{C} 18$ column (Welch Materials Inc.). The HPLC-DAD system was operated in $1.0 \mathrm{~mL} \cdot \mathrm{min}^{-1}$ flow rate and $20 \mu \mathrm{L}$ sample injection. The DAD was set to $\lambda$ of $265 \mathrm{~nm}$ and 
$620 \mathrm{~nm}$ for simultaneously detection of $\mathrm{MGOH}$ and MG respectively, with continuously full spectrum scans from 200 to $800 \mathrm{~nm}$. Fresh MG in binding buffer $(30 \mu \mathrm{M})$ was injected at $0 \mathrm{~h}$ and $24 \mathrm{~h}$, while $\mathrm{MGOH}$ in HPLC grade ACN $(30 \mu \mathrm{M})$ as a standard was injected at $0 \mathrm{~h}$. Elution profile for liquid chromatography was: 50\% mobile phase A (25 $\mathrm{mM}$ ammonium acetate, $\mathrm{pH} 6.8$ )/50\% mobile phase B (ACN, HPLC grade) for 5 min, a gradient to $15 \% \mathrm{~A} / 85 \% \mathrm{~B}$ from 5 to $15 \mathrm{~min}$, a $15 \% \mathrm{~A} / 85 \% \mathrm{~B}$ from 15 to $18 \mathrm{~min}$, followed by a ramp back to initial conditions from 18 to $21 \mathrm{~min}$ and held for $4 \mathrm{~min}$ thereafter.

\subsubsection{UV-Vis Spectral Study on Kinetic Conversion between MG and MGOH}

MG and MGOH were dissolved in binding buffer ( $\mathrm{pH}$ 6.7) with or without $10 \%$ (v/v) organic additive, respectively. The sample was loaded in a quartz cuvette and measured in a Varian Cary 50 UV-Vis spectrophotometer. The UV-Vis spectra were recorded every $5 \mathrm{~min}$, with a total period of 12 hours. MG $\lambda_{\max }$ at $618 \mathrm{~nm}$ was employed to calculate the change of MG. The decrease of MG in conversion $\mathrm{MG} \rightarrow \mathrm{MGOH}$ and the increase of $\mathrm{MG}$ in conversion $\mathrm{MGOH} \rightarrow \mathrm{MG}$ were fitted using first order equation which were performed by OriginPro 9.1.

\subsubsection{Electrophoresis of MGA}

Electrophoresis measurement was applied to analyze the integrity of MGA (RNA) in binding buffer over 48 hours. Samples stained with AMRESCO EZ-Vision® One (with amaranth indicator) were loaded on agarose gel (2\%), and electrophoresed for 40 minutes at $100 \mathrm{~V}$ in $1 \mathrm{X}$ tris-acetate-EDTA (TAE) buffer $(\mathrm{pH}=8.0)$. BioLabs $100 \mathrm{bp}$ DNA ladder (from $100 \mathrm{bp}$ to $1517 \mathrm{bp}$ ) was used as marker for reference. Finally, $365 \mathrm{~nm}$ UV exposure was applied to visualize the gel.

\subsection{Results and Discussion}


Prior to the test of the hypothesis, it is critical to get optimal conditions for reliable MGA/MG binding. Initially, $\mathrm{pH}$ effect was studied to obtain a suitable $\mathrm{pH}$ (6.7) for the binding according to our results and literature [6]. Particularly, owing to the known instability of MG and RNA, it is worth noting that the MG, MGA or MGA/MG binding may become unstable within a certain period. However, the fluorescence intensity and apparent $K_{\mathrm{d}}$ of MGA/MG binding is seldom reported. Wang et al. has experimentally and theoretically reported that MGA could prevent hydrolysis of MG in aptamer binding by monitoring the absorbance maxima in bound and free $\mathrm{MG}$, but no fluorescence data was mentioned [7]. According to our study on MGA/MG complex, fluorescence intensity decreased while apparent $K_{\mathrm{d}}$ increased as a function of time. For the first time, we report how the addition of common organic additives retained both fluorescence intensity and binding affinity, and thus stabilized the binding (within a 24 hour timeframe). This stabilization is beneficial for MGA-based applications towards accurate and repeatable detection assays. A synergistic stabilization mechanism based on kinetic analysis is proposed. Additionally, stability of MGA and MG was investigated by electrophoresis and HPLC respectively. All the results obtained in this chapter illustrate optimal experimental conditions for MGA/MG binding as well as the hypothesis testing.

\subsubsection{Effect of pH on Aptamer Binding Affinity}

Considering the possible impact of $\mathrm{pH}$ on aptamer binding and $\mathrm{MG}$, binding assays were tested in binding buffer with $\mathrm{pH}$ from 5 to 8.5. The results (Figure 4.2A and Figure 4.2B) suggest that the suitable range for binding is from $\mathrm{pH} 6$ to $\mathrm{pH} 8.5$, which results in $K_{\mathrm{d}}$ close to $200 \mathrm{nM}$. Thus, $\mathrm{pH}$ at 6.7 was chosen for the MGA/MG binding assay and hypothesis testing, which has been reported previous [6]. 

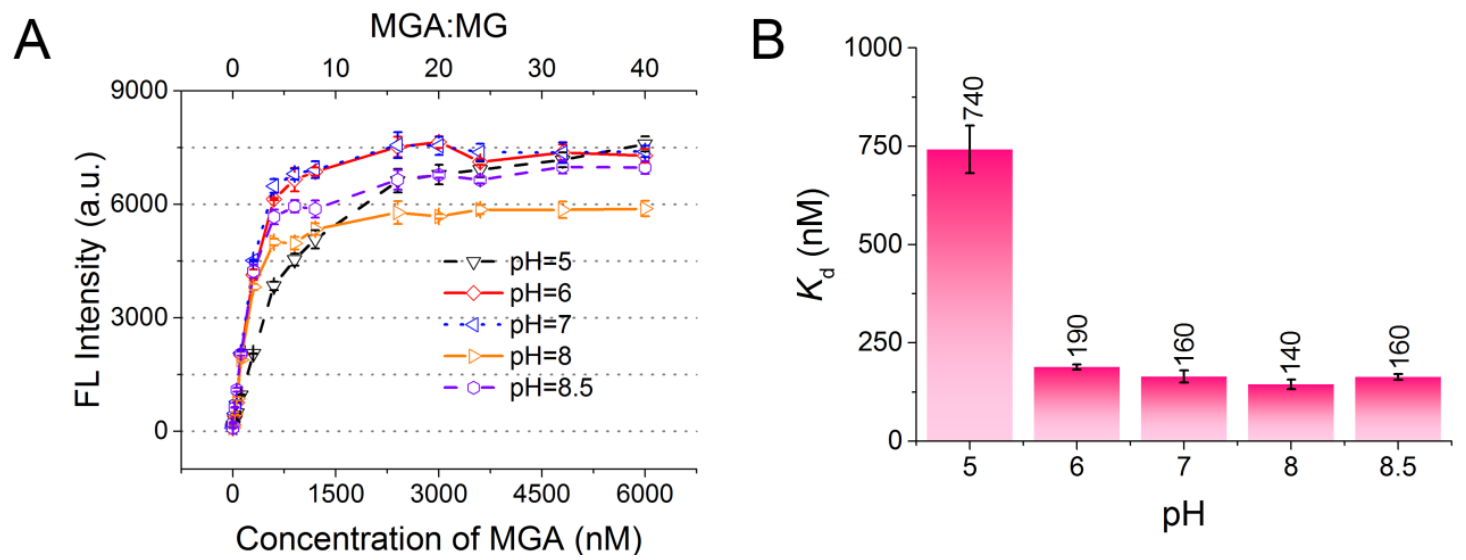

Figure 4.2 MGA/MG binding curves at ex/em $=620 \mathrm{~nm} / 656 \mathrm{~nm}(\mathrm{~A})$ and corresponding apparent $K_{\mathrm{d}}$ values (B) in various $\mathrm{pH}$. (Reprinted with permission from reference [4].)

\subsubsection{Fluorescence Intensity and Apparent Affinity of MGA/MG Complex Diminish Over Time}

As displayed in Figure 4.3A, the initial $K_{\mathrm{d}}$ is determined to be $200 \mathrm{nM}$ based on the MG fluorescence enhancement upon aptamer binding, which compares well to the result reported by Costa et al [6]. However, as presented in Figure 4.3A, fluorescence intensity and apparent binding affinity of $\mathrm{MGA} / \mathrm{MG}$ complex reduce with time, but reach a plateau at $5 \mathrm{~h}$. At $24 \mathrm{~h}, 40.8 \%$ of the fluorescence intensity at MGA:MG of 40:1 (maximum ratio) remains and the apparent $K_{\mathrm{d}}$ increases to be more than $10000 \mathrm{nM}$. The apparent $K_{\mathrm{d}}$ was determined based on the unique fluorescence enhancement of MG upon induced aptamer binding, but when the fluorescence signal decays, the apparent $K_{\mathrm{d}}$ will be directly affected. The fluorescence intensity at MGA:MG of 40:1 undergoes a first order decay over 24 hours with decay rate constant $k=2.5 \times 10^{-4} \mathrm{~s}^{-1}$ (Figure 4.3B). This decrease suggests an unstable association between MGA and MG, where more results will be presented to find out the cause below. Minute variations in $\mathrm{pH}$ are also ruled out since only minor shifts in $K_{\mathrm{d}}$ are observed across $\mathrm{pH} 6$ to 8.5 (Figure 4.2B), These results indicate an instable binding, which may be due to the loss of MGA or MG over time. This instability may affect the hypothesis testing and thus it is critical to find a way to prevent this instable binding. 

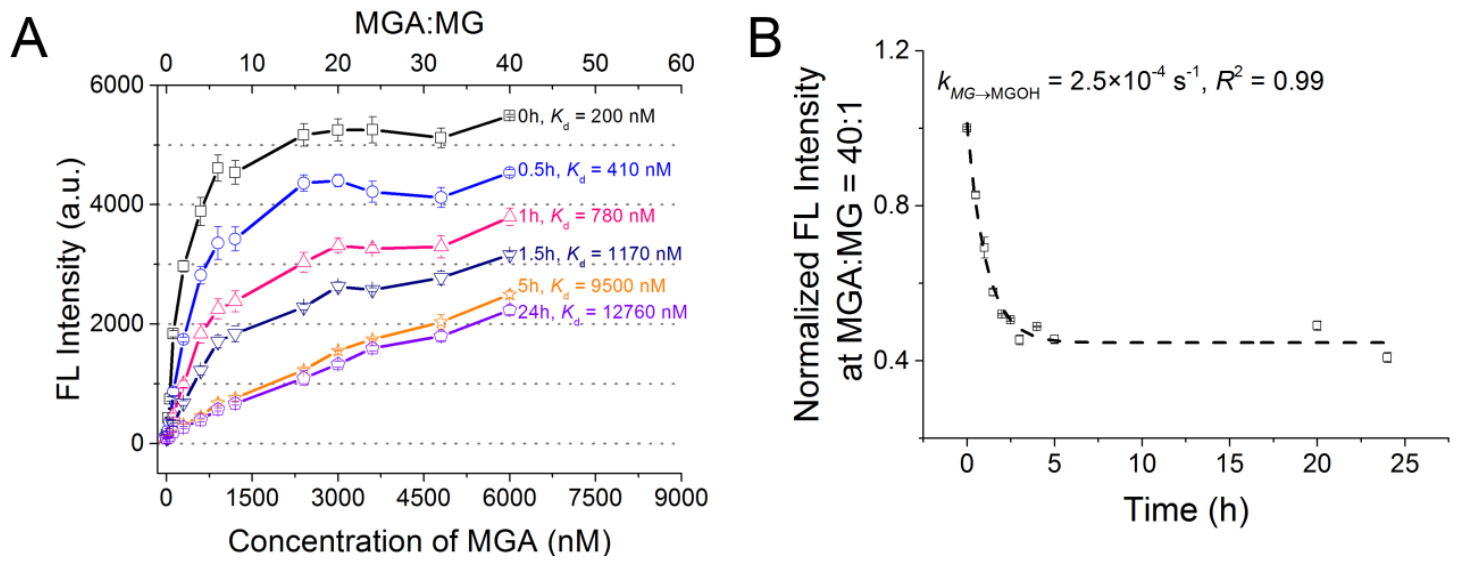

Figure 4.3 (A) Binding $K_{\mathrm{d}}$ of MGA/MG in binding buffer from 0 to $24 \mathrm{~h}$. (B) Normalized fluorescence decay at MGA:MG of 40:1 (the highest ratio). (Reprinted with permission from reference [4].)

\subsubsection{Addition of MG Reverses Decreased Fluorescence and MGA Keeps Stable Over Time}

In two individual preliminary tests, $\mathrm{MG}$ and MGA were separately added into the MGA/MG complex after 24 hours respectively to investigate the cause of fluorescence decay. From the results shown in Figure 4.4A, the presence of fresh MG recovers the fluorescence intensity instantly, which continues to decay. However, the presence of fresh MGA displays no recovery on fluorescence. Such observation indicates the decrease in fluorescence intensity was owing to events centered on MG rather than MGA, indirectly ruling out RNA aptamer degradation or misfolding.

To isolate the cause on fluorescence diminishing over time, stability of MGA (RNA aptamer) in binding buffer was investigate. To analyze the integrity of MGA over time, gel electrophoresis on MGA was conducted. As observed in Figure 4.4B, no obvious change in the MGA band is observed from $0 \mathrm{~h}$ to $48 \mathrm{~h}$. The kept integrity of MGA over $48 \mathrm{~h}$ enables its flexible use in aptamer binding for the hypothesis testing. 


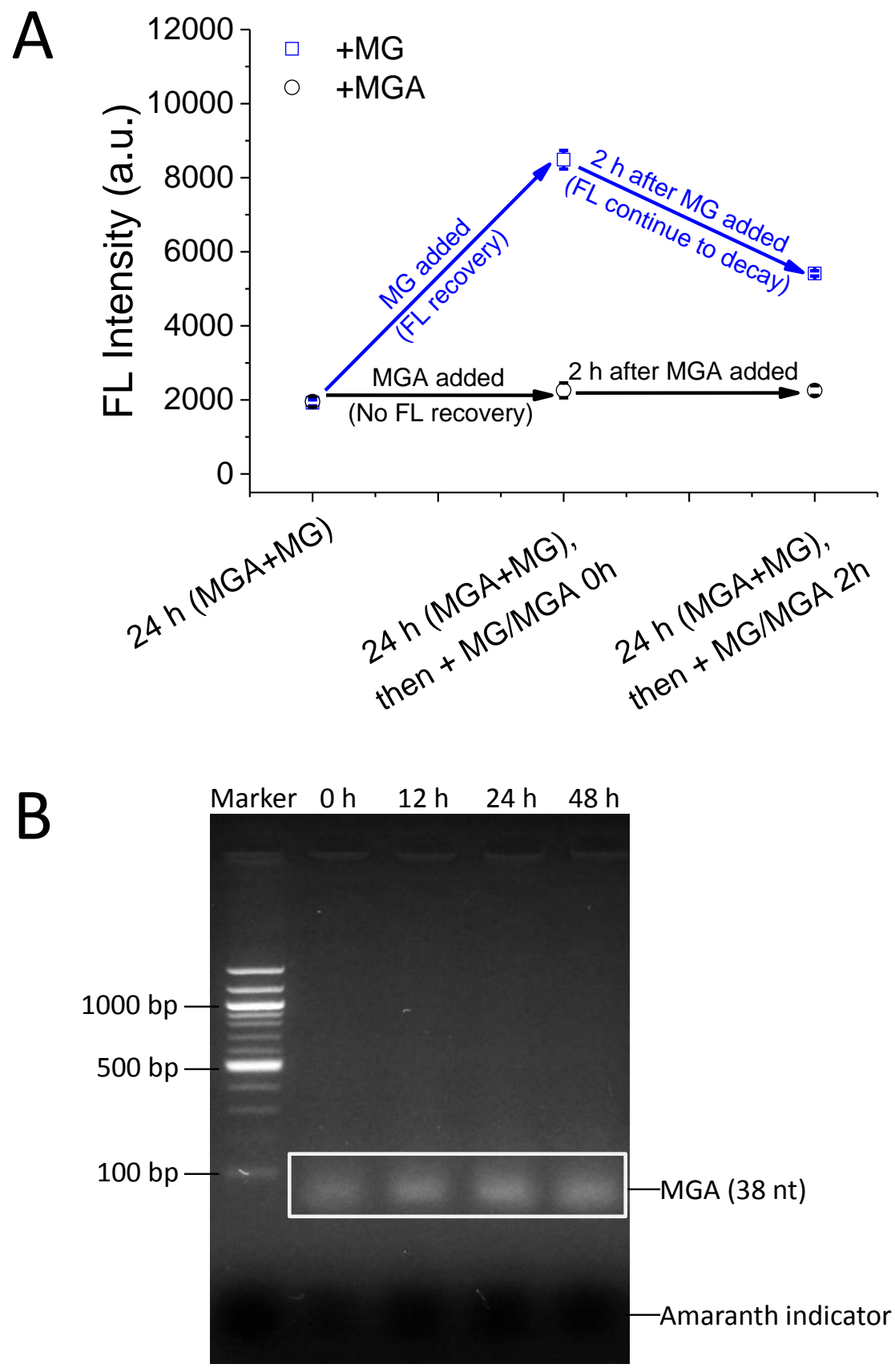

Figure 4.4 (A) Fluorescence intensity of MGA/MG complex after $24 \mathrm{~h}$ binding and with the subsequent addition of original amount of MG (blue square) and MGA (black circle). (B) Agarose gel electrophoresis of MGA over time in binding buffer. (Reprinted with permission from reference [4].) 


\subsubsection{Loss of Fluorescence Is Attributed to Hydrolysis of MG to MGOH}

HPLC-DAD allows the identification and quantification of MG derivatives through their chromatography and absorbance spectra. Figure 4.5A\&B insets present the UV-Vis spectra of MG and MGOH obtained by the DAD. HPLC chromatogram overlays of MG $0 \mathrm{~h}, \mathrm{MG} 24 \mathrm{~h}, \mathrm{MGOH}$ standard are shown in Figure 4.5A (with $\lambda=620 \mathrm{~nm}$ for MG) and Figure 4.5B (with $\lambda=265 \mathrm{~nm}$ for $\mathrm{MGOH}$ ). MG 0 h shows a major peak at $11.1 \mathrm{~min}$ with a minor peak at 18.2 min observed, attributed to $\mathrm{MGOH}$. The peak of $\mathrm{MGOH}$ in the $\mathrm{MG}$ $0 \mathrm{~h}$ sample presents $19 \%$ AUC compared to the $\mathrm{MGOH}$ standard $(30 \mu \mathrm{M})$. In other words, $5.7 \mu \mathrm{M}$ of $\mathrm{MGOH}$ is detected to exist in the $30 \mu \mathrm{M}$ of $\mathrm{MG} 0 \mathrm{~h}$ sample (as prepared). After $24 \mathrm{~h}$, the MG peak AUC decreases 36.3\% (Figure 4.5A) while the MGOH peak AUC increases 38.6\% (Figure 4.5B), nearly the same variation in AUC. In the MG $24 \mathrm{~h}$ sample, $\mathrm{MGOH}$ concentration nearly triples to $17.1 \mu \mathrm{M}$, and there is no observed formation of other minor peaks during 0-25 min elution. In summary, according to the HPLC results, only MG and MGOH are the major reactants and products in the binding condition. The 1 to 1 shift of MG peak to $\mathrm{MGOH}$ peak associated with the UV-Vis profile spectra confirms the conversion of $\mathrm{MG}$ to $\mathrm{MGOH}$, with no other peaks of interests detected $[1,2,8]$, nullifying other side reactions. This discovery confirms the cause of the instable MGA/MG binding, providing clue on how to stabilize the binding, which is beneficial for the hypothesis testing. 

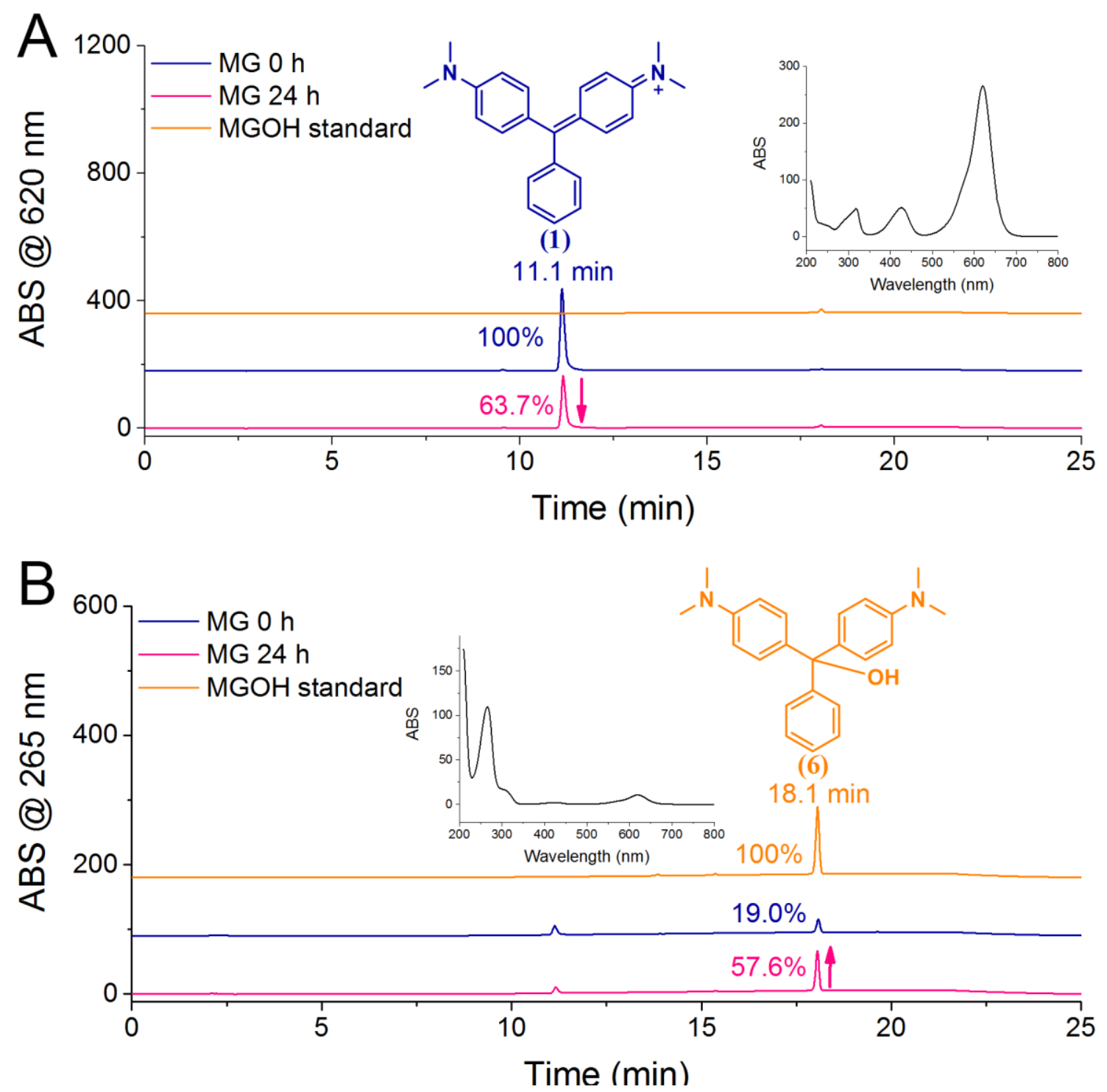

Figure 4.5 HPLC of MG 0h, MG $24 \mathrm{~h}$ and MGOH. (A) Detection $\lambda=620 \mathrm{~nm}$. (B) Detection $\lambda=265 \mathrm{~nm}$. (Reprinted with permission from reference [4].)

\subsubsection{Organic Additives Retain Fluorescence Intensity and Binding Affinity of MG with Limited Impact on $\boldsymbol{K}_{\mathrm{d}}$}

Binding buffer with different percentages (3\%-10\%, v/v) of acetonitrile (ACN), dimethyl sulfoxide (DMSO) or ethanol (EtOH) (organic additives usually applied in biological recognition for solubility enhancement) is mixed into the binding experiments of MGA with $\mathrm{MG}$, and the binding at $0 \mathrm{~h}$ are analyzed (Figure 4.6). Interestingly, there is observable increase in MGA/MG fluorescence across the highest MGA:MG ratio in the 
presence of organic additives (Figure 4.6A-C). In the presence of organic additives, more than 9000 fluorescence intensity unit are observed at MGA concentration above $3000 \mathrm{nM}$ (Figure 4.6A-C), where the 5500 fluorescence intensity at time $0 \mathrm{~h}$ in Figure 4.3A is about $40 \%$ lower. This raises the possibility that $\mathrm{MG} \rightarrow \mathrm{MGOH}$ conversion (Figure 4.5) exists even before the time $0 \mathrm{~h}$ binding measurements in Figure 4.3A were conducted, and the presence of organic additives shifts the conversion equilibrium constant, decay kinetics, or both. Further analysis will be presented in the following sections. Organic additives are known to increase the $K_{d}$ of molecular recognition elements [9]. To quantitate the impact from organic additives on binding affinity, the apparent $K_{\mathrm{d}}$ values are calculated and presented in Figure 4.6D. Only ACN weakens the overall $K_{d}$, while DMSO and EtOH show little to no changes in $K_{\mathrm{d}}$. Furthermore, the apparent $K_{\mathrm{d}}$ shows a strong correlation with percentage of $\mathrm{ACN}$, but weak to moderate correlation with percentage of DMSO or $\mathrm{EtOH}$, as seen in Figure 4.6D. In other words, the involvement of DMSO and EtOH results in little or no impact on $K_{\mathrm{d}}$, while $\mathrm{ACN}$ slightly weakens the overall apparent binding affinity, and the lowered affinity was proportional to the concentration of additives. Although the aptamer binding is typically conducted in aqueous environments, these results indicate the availability of organic additives in the $\mathrm{MGA} / \mathrm{MG}$ binding assay. In particular, it is beneficial for the employment of stilbene or its conjugates with poor aqueous solubility, facilitating their MGA binding based assay in the hypothesis testing. 
A

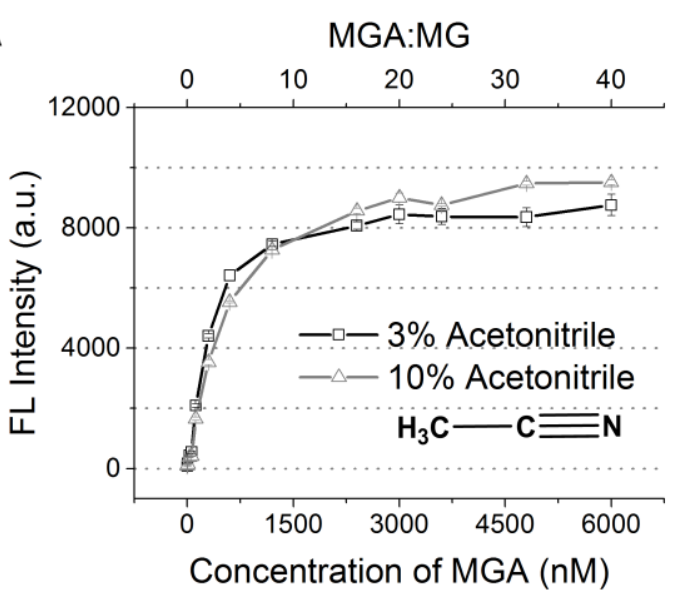

C

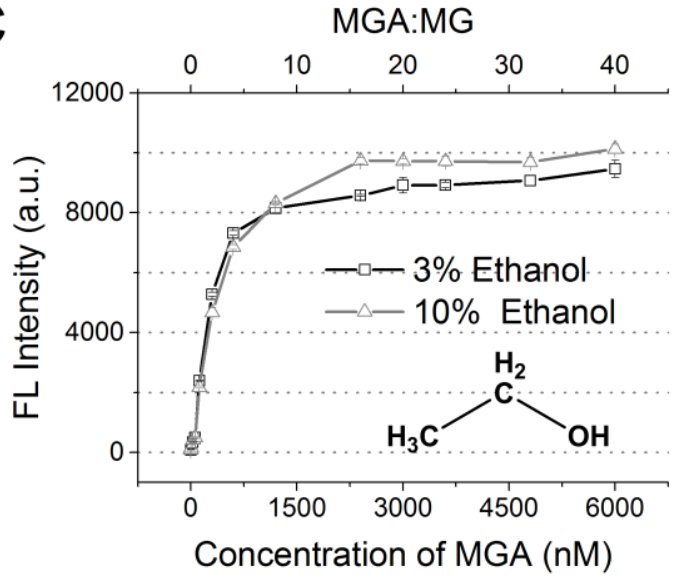

B

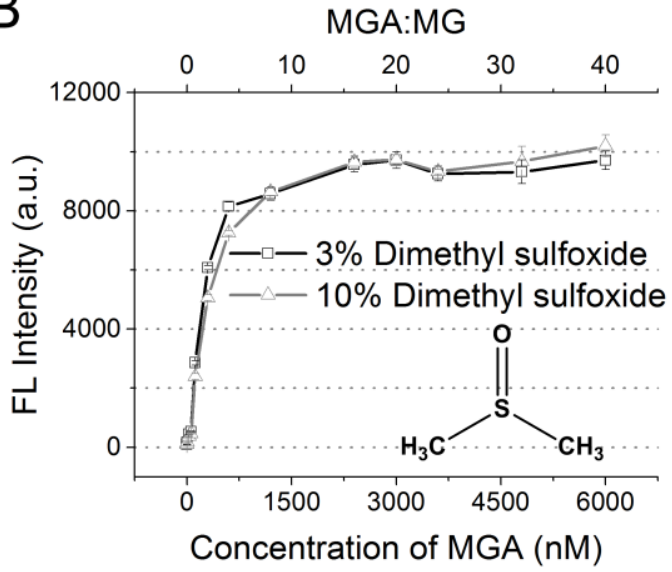

D

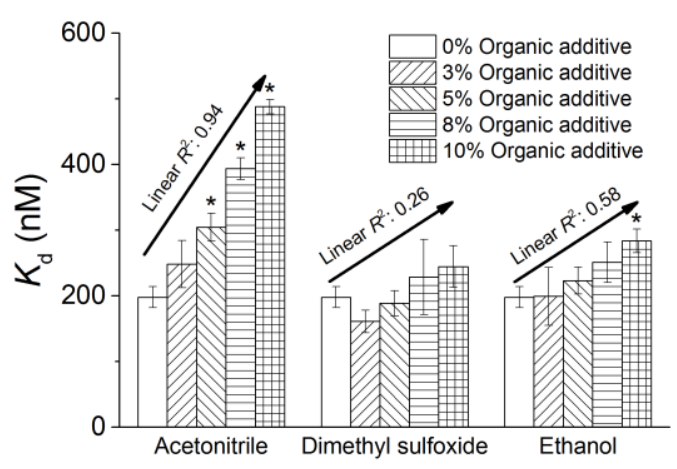

Figure 4.6 Apparent $K_{d}$ values with organic additives at time 0 h. (A) With 3\% and $10 \%$ ACN. (B) With 3\% and 10\% DMSO. (C) With 3\% and 10\% EtOH. (D) Variation of the apparent $K_{\mathrm{d}}$ values in different percentages of organic additives. ${ }^{*}=p<0.05$ : significant difference against apparent $K_{\mathrm{d}}$ without organic additive. (Reprinted with permission from reference [4])

The stability of MGA/MG binding after $24 \mathrm{~h}$ in the organic additive-buffer systems is investigated to see the effect of organic additives on the decreasing fluorescence intensity and binding affinity. Figure 4.7A illustrates the MGA/MG binding curves at $0 \mathrm{~h}$ and $24 \mathrm{~h}$ in the presence of different concentrations of ACN. Similar trends are found for DMSO and $\mathrm{EtOH}$ (data not shown). Figure 4.7B\&C summarize the apparent $K_{\mathrm{d}}$ and fluorescence intensity at $\mathrm{MGA}: \mathrm{MG}=40: 1$ at $0 \mathrm{~h}$ and $24 \mathrm{~h}$ obtained against concentrations organic additives. More fluorescence intensity and apparent binding affinity are remained as the concentration of organic additive increases, suggesting that the organic additives prevent hydrolysis and stabilize binding, which may be the result of 
a change in MG conversion $K_{\mathrm{eq}}$, decay kinetics or both. For instance, the fluorescence intensity at MGA:MG $=40: 1$ decreases to $40.8 \%$ compared to the initial value and the apparent $K_{\mathrm{d}}$ increases to ca. 65 -fold after $24 \mathrm{~h}$ binding (Figure 4.3A\&B). By contrast, as displayed in Figure 4.7B\&C, a 2.7-fold decrease in fluorescence decay $(21.7 \%$ vs. $59.2 \%)$ occurs with an impact on apparent $K_{\mathrm{d}}$ increasing being significant ( 3.2-fold vs. 65-fold compared to time 0) in the presence of $3 \%$ DMSO, where this impact is more apparent as the DMSO percentage increases. ACN and EtOH display the similar effect. Furthermore, organic additives lead to considerable shifts on the fluorescence decay kinetics (at MGA:MG $=40: 1$ ) as shown in Table 4.1. The first order decay kinetics of fluorescence decreased as the organic additive percentage increased from $3 \%$ to $10 \%$, which implies that organic additives shifted the $K_{\text {eq }}(\mathrm{MG} \leftarrow \rightarrow \mathrm{MGOH})$ towards $\mathrm{MG}$, and thus increased the overall amount of the fluorescent MGA/MG complex. The binding interactions between RNA aptamers and ligands are dynamic without a typical 'lock and key' static mechanism of enzyme, but follows an 'induced fit' dynamic mechanism that more closely resembles RNA folding surrounding its ligand, where both the RNA and ligand exhibits structural induced changes [10]. Thus, a clear explanation on how RNA folding varies after ligand hydrolysis hasn't been explored, but brings a high level complexity best removed if possible, and this is another reason for the employment of organic additives herein.

The fluorescence intensity loss and $K_{\mathrm{d}}$ increase were prevented upon the addition of organic additives. This observation provides a simple approach to stabilize aptamer binding, which is beneficial for applications relying on MGA for accurate MG sensing. The strategy may also be employed to other triphenylmethane compounds susceptible to hydrolysis. [11-14]. According to SciFinder, more than 100000 triphenylmethane compounds $\left((\mathrm{Ph})_{3}-\mathrm{C}\right)$ and 9000 substructures of MG have been reported to date. However, some triphenylmethane compounds have been banned for the use of farming owing to their potential toxicity, thus the monitoring of triphenylmethane compounds is of consumer significance [15]. Additionally, the triphenylmethane and other triarylmethane structures may form carbocations, which is susceptible to nucleophilic attack (i.e. hydrolysis in aqueous media). The discovery by using organic additives paves a way for 
stabilizing aptamer binding to ligands which have triphenylmethane or similar carbocation structures. Moreover, the decreasing trend of fluorescence decrease rate constants with increasing additive concentration (Table 4.1) displays a strong correlation. From another view, the rate constant may be tuned by the type and concentration of organic additives, which may beneficial for alternative designs for flow or in-line biosensors, for instance, sensing within specific time frames. These results indicate organic additives result in more $\mathrm{MG}$ and stabilize its aptamer binding as well as fluorescence intensity, providing optimal conditions for MGA/MG binding in the hypothesis testing.

Table 4.1 Decay rate constant $\left(k_{\mathrm{MG}_{\rightarrow} \mathrm{MGOH}}\right)$ of fluorescence intensity at MGA:MG = 40:1 (maximum ratio) with various organic additive percentages.

\begin{tabular}{cc}
\hline Media & $\boldsymbol{k}_{\mathbf{M G}_{\rightarrow} \mathbf{M G O H}}\left(\times \mathbf{1 0}^{-4}\right)\left(\mathbf{s}^{-1}\right)$ \\
\hline 3\% Acetonitrile & $3.1 \pm 0.4$ \\
5\% Acetonitrile & $2.6 \pm 0.1$ \\
8\% Acetonitrile & $1.3 \pm 0.2$ \\
10\% Acetonitrile & $1.0 \pm 0.1$ \\
3\% Dimethyl sulfoxide & $2.7 \pm 0.6$ \\
5\% Dimethyl sulfoxide & $0.2 \pm 0.3^{*}$ \\
8\% Dimethyl sulfoxide & $<0.01, \mathrm{NSD}$ \\
10\% Dimethyl sulfoxide & $<0.01, \mathrm{NSD}$ \\
3\% Ethanol & $4.0 \pm 2.1$ \\
5\% Ethanol & $1.5 \pm 0.1$ \\
8\% Ethanol & $0.4 \pm 0.1$ \\
10\% Ethanol & $0.2 \pm 0.1$ \\
\hline
\end{tabular}

* Fitting coefficient of determination $R^{2}<0.6$.

NSD: No statistical difference was seen between 0 and $24 \mathrm{~h}$ FLU. 

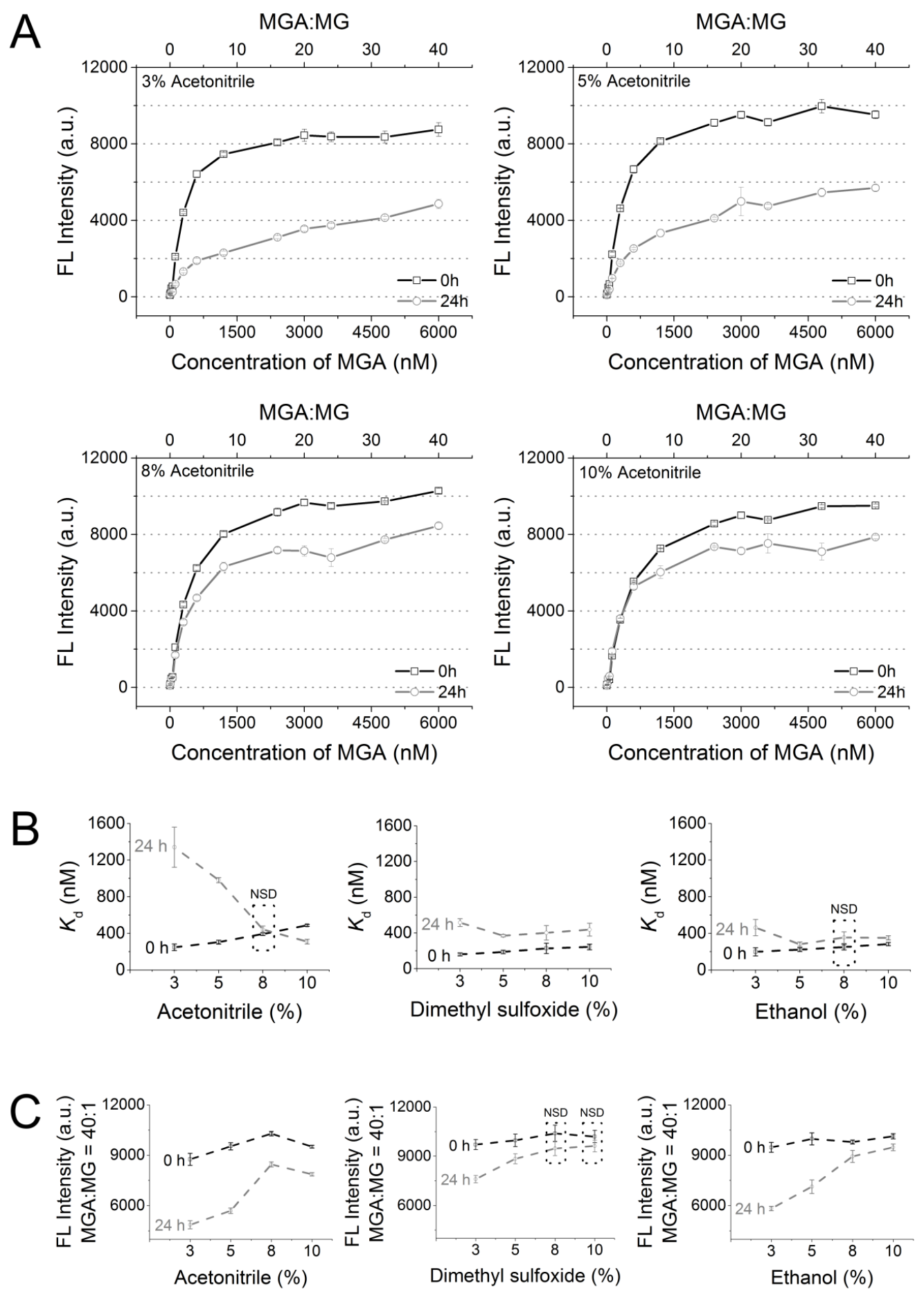

Figure 4.7 (A) Binding curves of MGA to MG with different percentage of ACN at $0 \mathrm{~h}$ and $24 \mathrm{~h}$. (B) Plots of calculated $K_{\mathrm{d}}$ values with different percentages of organic additives at $0 \mathrm{~h}$ and $24 \mathrm{~h}$. (C) Plots of fluorescence intensity at MGA:MG $=40: 1$ at $0 \mathrm{~h}$ and $24 \mathrm{~h}$ with different percentages of organic additives. NSD $=$ no statistical difference, $p<0.05$. (Reprinted with permission from reference [4]) 


\subsubsection{Organic Additives Dehydrate MGOH and Shift $K_{\text {eq }}$}

To further understand the MG hydrolysis, kinetics in both directions of $\mathrm{MG} \rightarrow \mathrm{MGOH}$ and $\mathrm{MGOH} \rightarrow \mathrm{MG}$ was evaluated independently without aptamer. The test on MG $\leftarrow \rightarrow$ MGOH conversion kinetics (in the absence of aptamer) with and without organic additives $(10 \% \mathrm{v} / \mathrm{v}$ ACN, DMSO or EtOH) is investigated by following the MG lambda max, as shown in Figure 4.8. The results is similar as noted in the HPLC characterization in Figure 4.5, that MG cannot be prepared pure in aqueous media owing to a $t_{1 / 2}$ of $\sim 19$ $\min \left(\ln 2 / 6.2 \times 10^{-4} \mathrm{~s}^{-1}\right)$, while MGOH can with $\mathrm{t}_{1 / 2}=2.8 \mathrm{~h}$. In binding buffer without any organic additive, the $\mathrm{MG} \rightarrow \mathrm{MGOH}$ conversion occurs 9-fold faster (Figure 4.8A, $k_{\mathrm{MG}_{\rightarrow} \mathrm{MGOH}}=6.2 \times 10^{-4} \mathrm{~s}^{-1}$ ) than the $\mathrm{MGOH} \rightarrow \mathrm{MG}$ conversion (Figure 4.8B, $k_{\mathrm{MGOH}_{\rightarrow} \mathrm{MG}}=$ $0.69 \times 10^{-4} \mathrm{~s}^{-1}$ ). Moreover, the MG $\rightarrow$ MGOH conversion reaches equilibrium after $2 \mathrm{~h}$, while the $\mathrm{MGOH} \rightarrow \mathrm{MG}$ conversion does not reach equilibrium after $12 \mathrm{~h}$, as shown in Figure 4.8B. When $\mathrm{MG}$ converts to $\mathrm{MGOH}$ with $10 \%$ organic additives (Figure 4.8A), the $k_{\mathrm{MG}_{\rightarrow} \mathrm{MGOH}}$ is retarded by $23 \%, 9 \%$, and $5 \%$ for ACN, DMSO, and EtOH, respectively. From the fluorescence intensity decay kinetics, the aptamer without additives retards the $k_{\mathrm{MG}_{\rightarrow} \mathrm{MGOH}}$ by $60 \%$ (Figure 4.3B and Figure 4.8A). These results suggest aptamer retards the $k_{\mathrm{MG}_{\rightarrow} \mathrm{MGOH}}$ while organic additives have not huge impact on $k_{\mathrm{MG}_{\rightarrow} \mathrm{MGOH}}$.

Surprisingly, the $\mathrm{MGOH} \rightarrow \mathrm{MG}$ conversion with $10 \%$ organic additives (Figure 4.8B) presents a comparably big shift in $k_{\mathrm{MGOH}_{\rightarrow} \mathrm{MG}}$. Organic additives increase $k_{\mathrm{MGOH}_{\rightarrow} \mathrm{MG}}$ (over pure buffer) by $360 \%, 450 \%$, and $460 \%$ for $\mathrm{ACN}, \mathrm{DMSO}$, and $\mathrm{EtOH}$, respectively. This acceleration on the conversion of $\mathrm{MGOH} \rightarrow \mathrm{MG}$ by organic additives leads to a large shift in $K_{\text {eq }}$ compared to the MG in buffer or (MGA + buffer). To investigate if the MGA impact $k_{\mathrm{MGOH}} \rightarrow \mathrm{MG}$, MGA were mixed with freshly prepared $\mathrm{MGOH}$ and the fluorescence of generated MGA/MG complex was monitored over time using different MGOH:MGA ratios. As displayed in Figure 4.9, at high ratios of 20:1 and 40:1, there is no statistical difference in fluorescence rate of MG formation with MGA, compared to the UV-Vis absorbance rate of MG formation without aptamer. In other words, high ratios of aptamer have no effect on $k_{\mathrm{MGOH}_{\rightarrow} \mathrm{MG}}$. Thus, the assumption that aptamer affected $\mathrm{MGOH} \rightarrow \mathrm{MG}$ 
conversion, was proven to be false, as increasing fluorescence (MG generation) was formed with the predicted kinetics. Combining the results of $\mathrm{MG} \rightarrow \mathrm{MGOH}$ conversion (Figure 4.8A), the results shows MGA retards $k_{\mathrm{MG}_{\rightarrow} \mathrm{MGOH}}$ while organic additives slow

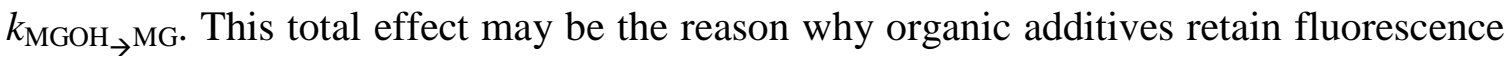
intensity and binding affinity of MG (Figure 4.7), where the $K_{\text {eq }}$ is shifted by the synergistic effect from both aptamer and organic additives. Towards MGA/MG binding at $0 \mathrm{~h}$ in Figure 4.6A-C, organic additives lower the affinity, but increase the fluorescence intensity of MGA/MG complex (compared to Figure 4.3A). This paradox can be explained by the $K_{\mathrm{eq}}$ shift and by noting that MG solutions (freshly prepared) always contain various ratios of $\mathrm{MG}$ to $\mathrm{MGOH}$ (Figure 4.5). Figure 4.8 illustrates the accelerating $\mathrm{MGOH} \rightarrow \mathrm{MG}$ conversion by organic additives. Hence, the presence of organic additives results in synergistic shift towards MG (with aptamer), thus enhances the formation of MGA/MG based fluorescence.
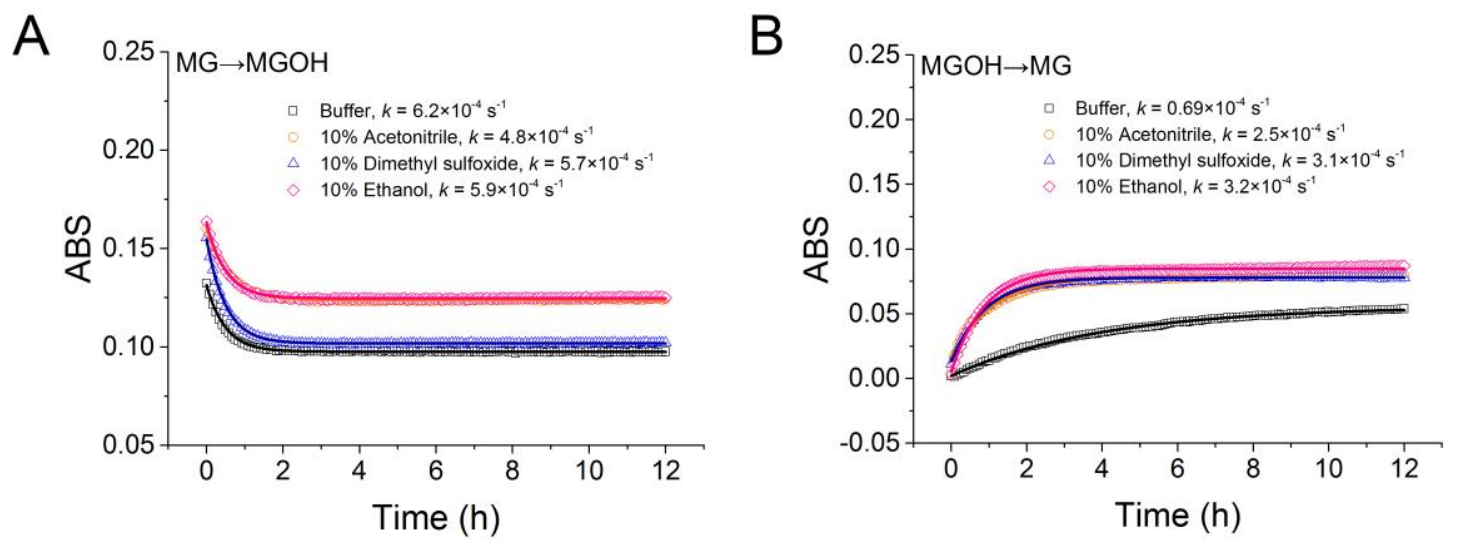

Figure 4.8 Kinetic evolution between $\mathrm{MG}$ and $\mathrm{MGOH}$ (without aptamer). (A) MG converts to $\mathrm{MGOH}$ in binding buffer and in the presence of $10 \%$ organic additives. (B) MGOH converts to MG in binding buffer and in the presence of $10 \%$ organic additives. (Reprinted with permission from reference [4]) 


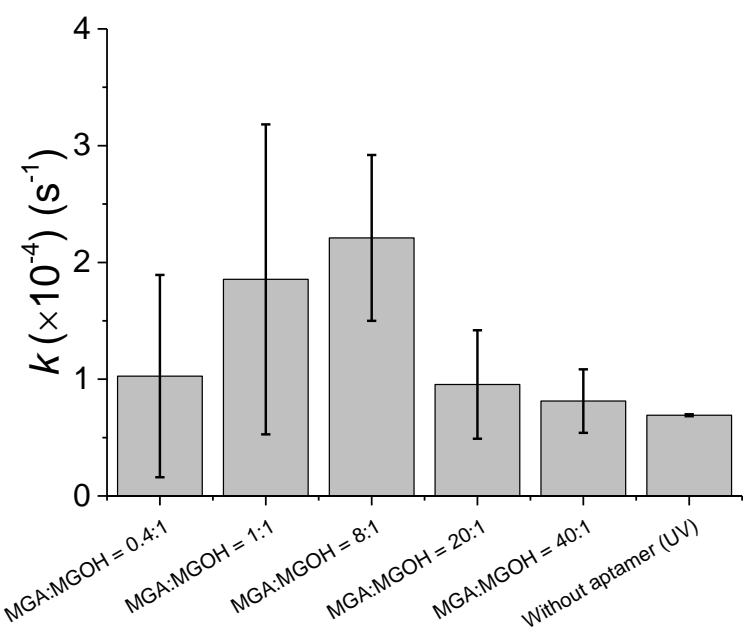

Figure 4.9 Rate constant of $\mathrm{MGOH}$ to $\mathrm{MG}\left(k_{\mathrm{MGOH}_{\rightarrow} \mathrm{MG}}\right)$ in binding buffer in the presence of MGA. (Reprinted with permission from reference [4])

\subsubsection{Organic Additives Reverse Decreased Fluorescence through MG Dehydration}

The results above demonstrate the stabilizing effect on fluorescence intensity of organic additives by dehydrating $\mathrm{MGOH}$. However, can the organic additives recover the decreased fluorescence intensity of MGA/MG complex? Thus, DMSO was added into MGA:MG buffer solutions which have reached a stable fluorescence intensity after $5 \mathrm{~h}$ of binding, and Figure 4.10 shows the fluorescence recovery of 3\% and 5\% DMSO after $1 \mathrm{~h}$ and $12 \mathrm{~h}$ incubation with the MGA/MG complex. The data suggests that organic additives can immediately vary the reaction kinetics to favor $\mathrm{MG}$ as the major equilibrium product. A minimum of $5 \% \mathrm{v} / \mathrm{v}$ DMSO is needed to recover the fluorescence intensity after $12 \mathrm{~h}$. Higher DMSO concentration can result in fluorescence intensity values of 10000 (at MGA:MG > 20:1), similar to the values displayed in Figure 4.7 (data not shown). The observation that the decreased fluorescence is completely reversible by the addition of organic additives (Figure 4.10) speculates that MG undergoes a time dependent variation in charge redistribution or electronic resonance within the binding site of MGA, which might reduce the quantum yield of MG or shift its fluorescent planar configuration formed by 'induced fit' $[3,10]$. We speculate the intermediate of $\mathrm{MG}$ hydrolysis, $\mathrm{MG}-\mathrm{OH}_{2}$, is responsible for the fluorescence decay through electronic or charge redistribution inside the binding pocket of aptamer [16], as the $\mathrm{C}^{+}-\mathrm{OH}_{2}$ 
carbocation is further stabilized and turns to the preferred resonance structure. Shifts in NMR peaks of $M G{ }^{13} \mathrm{C}$ have confirmed the charge distribution change between free and bound $\mathrm{MG}$ [10]. However, because ${ }^{13} \mathrm{C}$ measurements require long timescale, it is unlikely to distinguish MG intermediates from MG. Alternatively, the more hydrophilic $\mathrm{C}^{+}-\mathrm{OH}_{2}$ carbocation may weaken the RNA base stacking forces, relaxing the induced fluorescent planar configuration [17]. The employment of organic additives prevents or reverses this. This observation also extends the application window of MGA/MG binding for hypothesis testing, where organic additives can be employed for stabilizing the binding even after the MGA/MG binding has started.

A

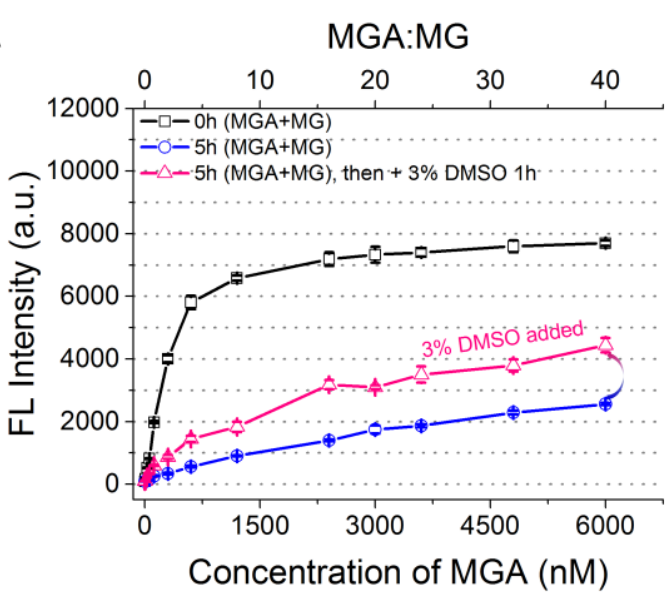

C

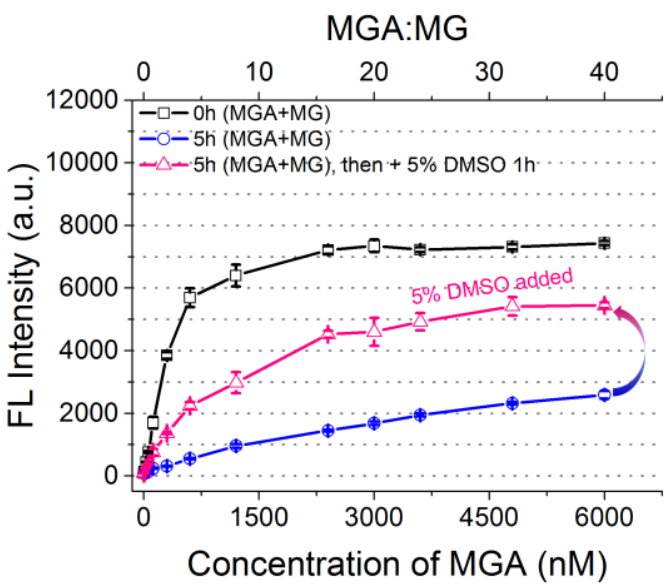

B

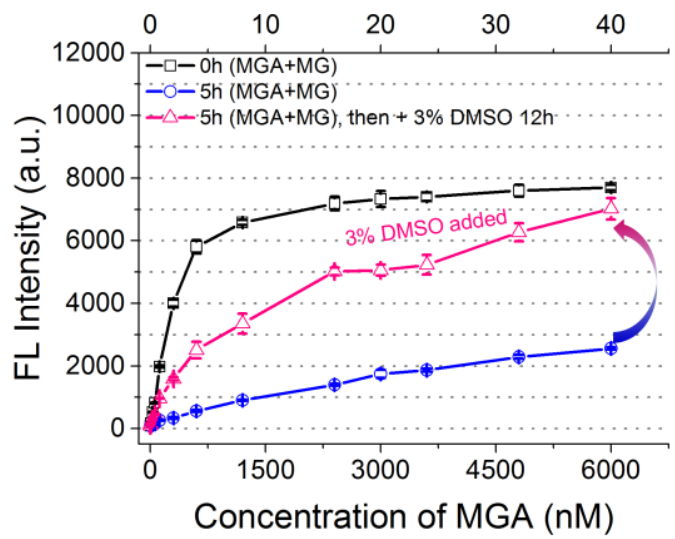

$\mathrm{D}$

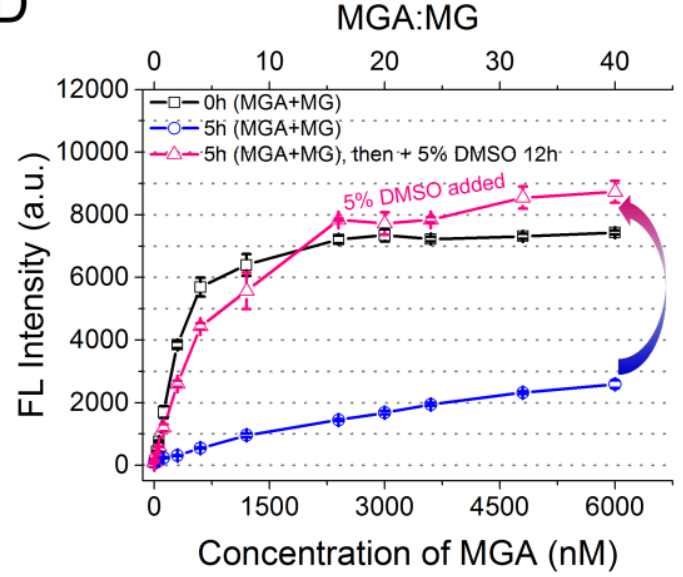

Figure 4.10 Addition of DMSO into MGA/MG complex at $5 \mathrm{~h}$. (A) $3 \% \mathrm{v} / \mathrm{v}$ DMSO for $1 \mathrm{~h}$. (B) 3\% v/v DMSO for $12 \mathrm{~h}$. (C) 5\% v/v DMSO for $1 \mathrm{~h}$. (D) 5\% v/v DMSO for $12 \mathrm{~h}$. (Reprinted with permission from reference [4]) 


\subsubsection{Synergistic Stabilization of Ligand by Aptamer and Organic Additives}

Table 4.2 summarizes the MG kinetic constant $\left(k_{\mathrm{MG}_{\rightarrow} \mathrm{MGOH}}\right)$ and $K_{\text {eq }}$ in various organic additives in the presence and absence of MGA, and lists the molecular properties of water and organic additives for evident correlations. In the absence of aptamer, organic additives only had a slight impact on the conversion of $\mathrm{MG} \rightarrow \mathrm{MGOH}$. However, the conversion was $60 \%$ slower with MGA, suggesting the protective effect from aptamer in terms of hydrolysis. More importantly, this protective effect combined with the organic additives exhibits a synergistic result towards a stable formation of fluorescent MGA/MG complex. The adjusted $k_{\mathrm{MG}_{\rightarrow} \mathrm{MGOH}}$ obtained by fluorescence intensity in binding buffer was 5 times faster than in buffer with $10 \% \mathrm{ACN}$ and 29.5 times fast than in buffer with $10 \% \mathrm{EtOH}$. No detectable conversion of MG was observed with 10\% DMSO, preventing the measurement of $k_{\mathrm{MG}_{\rightarrow} \mathrm{MGOH}}$ under the experimental conditions. In addition, Pearson's $R$ analysis implies the correlations between kinetic constant and intrinsic molecular properties of the organic additives. We speculate this may lead to the prediction of even more effective organic additives. In the absence of aptamer, no distinct correlation between kinetic constant and intrinsic properties of the organic additives is observed $(0.5>$ $R>-0.5)$. With aptamer, kinetic constant has a strong direct correlation with molecular polar surface area (MPSA) $(R>0.99)$ and a strong inverse correlation with volume $(R<-$ 0.99). The $10 \%$ DMSO is excluded in calculation because of its unavailable kinetic constant. However, the lack of fluorescence decay follows the strong correlation of the DMSO intrinsic molecular properties (small in MPSA and relatively large in volume). 
Table 4.2 MG hydrolysis rate constant $\left(k_{\mathrm{MG}_{\rightarrow} \mathrm{MGOH}}\right)$ with $10 \%$ of organic additives in the presence and absence of MGA (MGA:MG = 40:1), and the theoretical calculated properties of water and additives.

\begin{tabular}{|c|c|c|c|c|c|c|c|}
\hline \multirow[t]{2}{*}{ Media } & \multicolumn{2}{|c|}{ With Aptamer } & \multicolumn{2}{|c|}{ Without Aptamer } & \multirow[t]{2}{*}{ Solvent } & \multicolumn{2}{|c|}{$\begin{array}{l}\text { Molecular } \\
\text { Properties }^{\#}\end{array}$} \\
\hline & $\begin{array}{l}k_{\mathrm{MG}_{\rightarrow} \mathrm{MGOH}} \\
\left(\times 10^{-4}\right)\left(\mathrm{s}^{-1}\right)\end{array}$ & $K_{e q}$ & $\begin{array}{c}k_{\mathrm{MG}_{\rightarrow} \mathrm{MGOH}} \\
\left(\times 10^{-4}\right)\left(\mathrm{s}^{-1}\right)\end{array}$ & $K_{e q}$ & & $\begin{array}{c}\text { Molecular } \\
\text { Polar } \\
\text { Surface } \\
\text { Area } \\
(\text { MPSA) } \\
\left(\AA^{2}\right)\end{array}$ & $\begin{array}{l}\text { Volu } \\
\text { me } \\
\left(\AA^{3}\right)\end{array}$ \\
\hline buffer & $2.5 \pm 0.2$ & 3.62 & $6.2 \pm 0.1$ & 8.99 & Water & 29.3 & 19.33 \\
\hline $10 \%$ Acetonitrile & $1.0 \pm 0.1$ & 0.40 & $4.8 \pm 0.1$ & 1.92 & Acetonitrile & 23.8 & 46.05 \\
\hline $10 \%$ Ethanol & $0.2 \pm 0.1$ & 0.06 & $5.9 \pm 0.1$ & 1.84 & Ethanol & 20.2 & 54.02 \\
\hline $\begin{array}{l}\text { 10\% Dimethyl } \\
\text { sulfoxide }\end{array}$ & NSD* & $\begin{array}{c}< \\
0.05^{*}\end{array}$ & $5.7 \pm 0.0$ & 1.84 & $\begin{array}{l}\text { Dimethyl } \\
\text { sulfoxide }\end{array}$ & 17.1 & 71.43 \\
\hline $\begin{array}{c}\text { Pearson's } R \\
\text { correlation, } \\
k_{M G} \rightarrow M G O H \text { Vs. } \\
\text { MPSA }\end{array}$ & $+0.999^{* *}$ & & +0.189 & & & & \\
\hline $\begin{array}{c}\text { Pearson's } R \\
\text { correlation, } \\
k_{M G \rightarrow M G O H} \text { Vs. } \\
\text { Solvent Volume }\end{array}$ & $-0.991^{* *}$ & & -0.293 & & & & \\
\hline
\end{tabular}

* No statistical difference in 0 and $24 \mathrm{~h}$ FLU values under $10 \%$ dimethyl sulfoxide additive.

**: $10 \%$ Dimethyl sulfoxide is excluded in the correlation due to its unavailable kinetic constant.

\#: Calculated by Molinspiration Cheminformatics 2015: www.molinspiration.com.

The kinetic study results (Figure 4.8) suggest a relatively fast MG $\rightarrow$ MGOH hydrolysis reaction in binding buffer. The larger $k_{\mathrm{MG}_{\rightarrow} \mathrm{MGOH}}$ (compared to the one with aptamer binding in Figure 4.3B) shows the evidence that the aptamer is able to interfere with the hydrolysis of MG, without completely preventing. Wang et al. has argued MGA could prevent MG from hydrolysis well over time, owing to its steric hindrance effect [7]. Nonetheless, our results display that a weaker protective effect, which is likely because of the different experimental methods of affinity and kinetics evaluation. Wang et al. evaluated the hydrolysis of MG by monitoring MG's absorbance maxima changes, where we based ours on fluorescence measurement. Changes in absorbance maxima may not be 
apparent between aptamer complexed $\mathrm{MG}$ and the intermediate $\mathrm{MG}-\mathrm{OH}_{2}$, thus accounting for their perceived observation of full stopping of hydrolysis. If the bound intermediate $\mathrm{MG}-\mathrm{OH}_{2}$ has similar UV-Vis absorbance but is responsible for the decrease of fluorescence, Results from Wang's report provide further evidence that $\mathrm{MG}-\mathrm{OH}_{2}$ intermediate may still have enough binding affinity to keep the MGA folded, while $\mathrm{MGOH}$ has not. In the presence of aptamer, the organic additives exhibited a slight inhibition on $k_{\mathrm{MG}_{\rightarrow} \mathrm{MGOH}}$ (Figure 4.8A), but catalyze the $\mathrm{MGOH}$ dehydration to $\mathrm{MG}$ (Figure 4.8B). Employment of organic additive not only accelerated the $\mathrm{MGOH} \rightarrow \mathrm{MG}$ conversion, but the accelerated conversion appeared to shift the equilibrium in favor of MG (Table 4.2). This impact on kinetics and equilibrium defines the approach how the organic additives stabilized the MGA/MG complex fluorescence intensity over $24 \mathrm{~h}$.

By combining the rates changes caused by the organic additives and aptamer, synergistic effect of stabilization on MG was observed (Table 4.2). With aptamer, a significant correlation between rate constant and the molecular properties of the organic additives can be obtained by Pearson's $R$ analysis. Decreasing MG hydrolysis rate constants correlates with less MPSA, larger volume, or a combination of both. Nguyen et al. reported the conformation and charge distribution of MG can be changed upon MGA binding [10], which may result in a shift in kinetics and thermodynamics of MG hydrolysis. Nonetheless, to our knowledge, no report has been found on what the influence of organic additives may bring on the MG stability inside the binding pocket of aptamer. The results in this chapter suggest that the organic additives: 1) shift equilibrium towards MG with aptamer, 2) had minor effect on MG hydrolysis in the absence of aptamer, 3) catalyze the MGOH dehydration and 4) had only minor shifts on affinity and $K_{\mathrm{d}}$. Based on these primary four observations, a proposed mechanism is displayed in Figure 4.11. 
A<smiles>Cc1ccc(C(c2ccccc2)c2ccc(N(C)C)cc2)cn1</smiles>

(1)

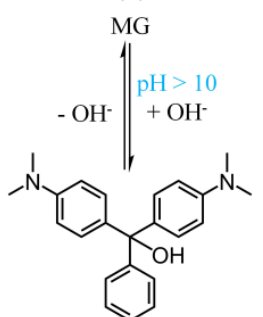

(6)

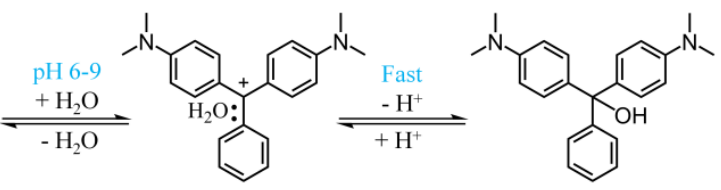

(7)

$\mathrm{MG}-\mathrm{OH}_{2}$

MGOH

B $\quad$ Rate $=\frac{-d[M G]}{d t}=[M G]\left(k_{\mathrm{H}_{2} \mathrm{O}}\left[\mathrm{H}_{2} \mathrm{O}\right]+k_{O H^{-}}\left[O \mathrm{H}^{-}\right]\right) \rightarrow k_{o b s}=k_{M G \rightarrow M G O H}$

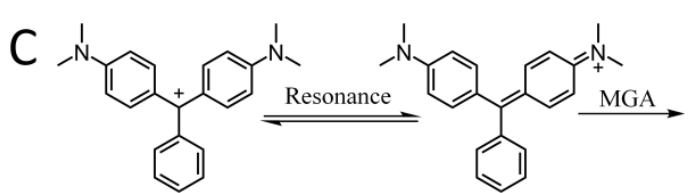

(8)
(1)

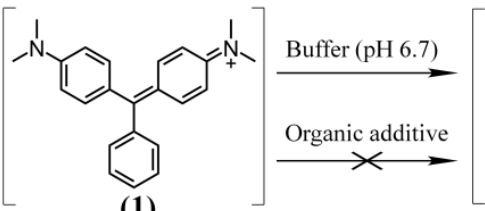

(1)

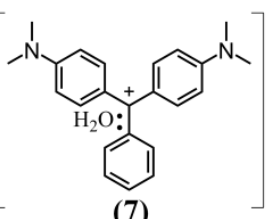

(7)

Free MG, not fluorescent Bound MG, fluorescent Bound MG-OH

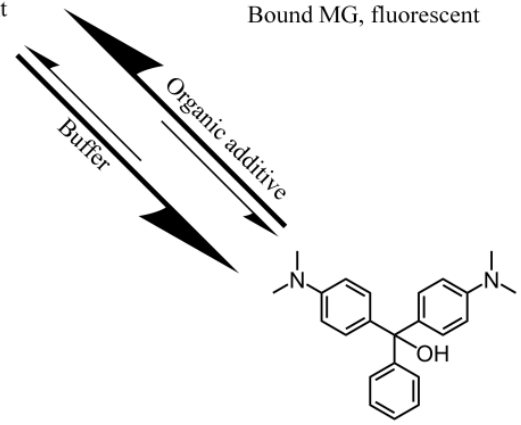

(6)

MGOH, no binding

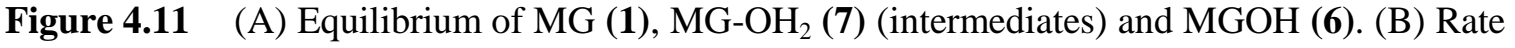
of MG elimination. At $\mathrm{pH} 6.7, k_{\mathrm{obs}} \approx k_{\mathrm{MG}_{\rightarrow} \mathrm{MGOH}}[16]$. (C) Proposed mechanism of the conversions between $\mathrm{MG}, \mathrm{MG}-\mathrm{OH}_{2}$ and $\mathrm{MGOH}$ with MGA presented. Without MGA, the rate constants $k_{\mathrm{MG}_{\rightarrow} \mathrm{MGOH}}$ (hydrolysis) and $k_{\mathrm{MGOH}} \rightarrow \mathrm{MG}$ (dehydration) favor $\mathrm{MGOH}$ as the major equilibrium product (A). When excess MGA is present, it retards MG hydrolysis, resulting in a decreased $k_{\mathrm{MG}_{\rightarrow} \mathrm{MGOH}}$. With the addition of organic additives, $k_{\mathrm{MGOH}_{\rightarrow} \mathrm{MG}}$ increases by few hundred percent (C), which results in a "stabilizing" effect. (Reprinted with permission from reference [4])

In $\mathrm{pH} 6.7$ binding buffer, $\mathrm{MG}$ hydrolyzes to $\mathrm{MG}-\mathrm{OH}_{2}$ (intermediate), which transforms to $\mathrm{MGOH}$ (Figure 4.11A). Thus, in the absence of aptamer, the rate constants $k_{\mathrm{MG}_{\rightarrow} \mathrm{MGOH}}$ (hydrolysis) and $k_{\mathrm{MGOH}_{\rightarrow} \mathrm{MG}}$ (dehydration) favor $\mathrm{MGOH}$ as the major equilibrium product. 
Additionally, as displayed in Figure 4.11B, $k_{\mathrm{obs}} \approx k_{\mathrm{MG}_{\rightarrow} \mathrm{MGOH}}$ in this $\mathrm{pH}$ [16]. Thus, as proposed in Figure 4.11C, when MGA is in excess of MG, it retards MG hydrolysis by slowing the $\mathrm{MG}-\mathrm{OH}_{2} \rightarrow \mathrm{MGOH}$ conversion, resulting in a decreased $k_{\mathrm{MG}_{\rightarrow} \mathrm{MGOH}}$ (summarized in Table 4.2). However, such inhibition by MGA is still not able to enable

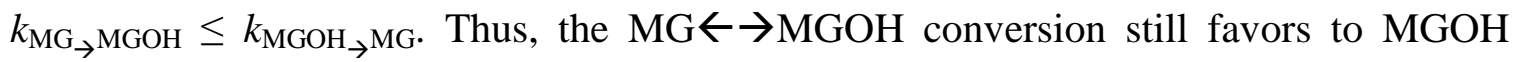
production, which can be evidenced from the result of fluorescence decrease of

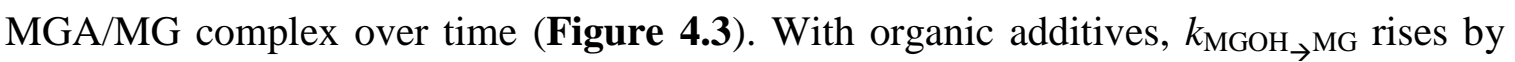

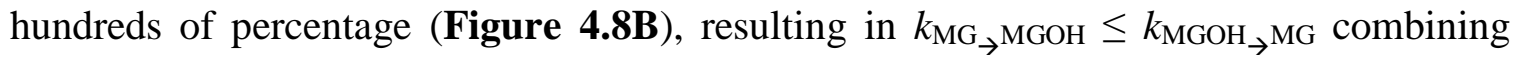
with the synergistic effect from MGA. This synergistic effect by both aptamer (decrease of $k_{\mathrm{MG}_{\rightarrow} \mathrm{MGOH}}$ ) and organic additives (increase of $k_{\mathrm{MG}_{\rightarrow} \mathrm{MGOH}}$ ) results in a global $K_{\text {eq }}$ favoring inhibition of MG hydrolysis (Figure 4.7).

\subsection{Conclusions}

It is critical to establish optimal conditions for MGA/MG binding, prior to the hypothesis testing. In this chapter, we investigated the factors that may affect the MGA/MG binding, like $\mathrm{pH}$, stability of MGA, MG and their binding. It was found that there was loss of fluorescence intensity and increase in apparent $K_{\mathrm{d}}$ in MGA/MG binding, which was due to the hydrolysis of MG into speculated intermediates and $\mathrm{MGOH}$. This phenomenon may affect our aptasensing assay in the proposed conjugate sensor according to our hypothesis. Interestingly, organic additives were found to prevent the fluorescence decrease (over time) and minimize shifts of apparent $K_{\mathrm{d}}$. A synergistic mechanism through both aptamer and organic additive on the prevention of carbocation hydrolysis is proposed. Various results and discoveries obtained in this chapter establish optimal conditions for hypothesis testing and provide useful information and foundation on the future aptameric sensing.

\section{References:}

[1] D. He; X. He; K. Wang; J. Cao; Y. Zhao Advanced Functional Materials 2012, 22, 4704. 
[2] B. Samiey; M. Zafari Journal of the Chinese Chemical Society 2012, 59, 32.

[3] J. R. Babendure; S. R. Adams; R. Y. Tsien Journal of the American Chemical Society 2003, 125, 14716 .

[4] Y. Zhou; H. Chi; Y. Wu; R. S. Marks; T. W. J. Steele Talanta 2016, 160, 172.

[5] J. Bernard Da Costa; T. Dieckmann Mol Biosyst 2011, 7, 2156.

[6] J. B. Da Costa; A. I. Andreiev; T. Dieckmann Biochemistry 2013, 52, 6575.

[7] T. Wang; J. A. Hoy; M. H. Lamm; M. Nilsen-Hamilton Journal of the American Chemical Society 2009, 131, 14747.

[8] S. Chen; Y. Jiang; Z. Wang; X. Zhang; L. Dai; M. Smet Langmuir 2008, 24, 9233.

[9] S. L. Stead; H. Ashwin; B. Johnston; A. Dallas; S. A. Kazakov; J. A. Tarbin; M. Sharman; J. Kay; B. J. Keely Anal Chem 2010, 82, 2652.

[10] D. H. Nguyen; S. C. DeFina; W. H. Fink; T. Dieckmann J Am Chem Soc 2002, 124, 15081 .

[11] C. Baugh; D. Grate; C. Wilson J Mol Biol 2000, 301, 117.

[12] L. García-Río; J. R. Leis; J. C. Mejuto; A. Navarro-Vázquez; J. Pérez-Juste; P. Rodriguez-Dafonte Langmuir 2004, 20, 606.

[13] Y. Wu; S. Zhan; H. Xing; L. He; L. Xu; P. Zhou Nanoscale 2012, 4, 6841.

[14] B. Samiey; Z. Dalvand International Journal of Chemical Kinetics 2014, 46, 60.

[15] W. C. Andersen; S. B. Turnipseed; C. M. Karbiwnyk; R. H. Lee; S. B. Clark; W. D. Rowe; M. R. Madson; K. E. Miller Analytica Chimica Acta 2009, 637, 279.

[16] B. M. Fox; G. Hallas; J. D. Hepworth; D. Mason Journal of Chemical Technology and Biotechnology 1980, 30, 317.

[17] J. Flinders; S. C. DeFina; D. M. Brackett; C. Baugh; C. Wilson; T. Dieckmann Chembiochem 2004, 5, 62 . 


\section{Chapter 5}

\section{Binding and Fluorescence Properties of Malachite Green- Stilbene Conjugates}

This chapter investigates the binding and fluorescence properties of synthetic malachite green $(M G)$-stilbene conjugates. Aptamer binding affinity and stilbene fluorescence decay are required parameters of $M G$ stilbene conjugates towards hypothesis testing and aptasensor design. Structure activity relationships were focused on conjugate covalent linkers and stilbene side groups. MG-stilbene conjugate with amide covalent linker and -methoxy stilbene electron donating groups provided the most efficient aptasensor design. 


\subsection{Introduction}

To examine the hypothesis according to design "competitive stilbene-analyte conjugate with analyte" (section 1.4.1), several conjugates of malachite green (MG) (1) and stilbene were analyzed towards aptamer binding and stilbene photoisomerization. The conjugate design attempts to retain MG's aptamer binding properties while incorporating and stilbene's fluorescence decay. Rhodamine B (RhB)-stilbene conjugate (11) was designed as a negative control, as RhB has no malachite green aptamer (MGA) binding. This rules out stilbene conjugation as a mechanism of non-specific aptamer binding. Binding of MGA to MG and fluorescence decay of stilbene molecule $\left(\mathrm{CH}_{3}\right)_{2}-\mathrm{N}$-stilbene$\mathrm{NH}_{2}$ (9) were employed as positive controls for each parameter. The chemical structures of the MG, RhB (10), $\left(\mathrm{CH}_{3}\right)_{2}-\mathrm{N}$-stilbene- $\mathrm{NH}_{2}$ and their conjugates are listed in Figure 5.1. The results show the MG-stilbene conjugate molecules possess either low binding affinity or weak fluorescence decay, which limits its design as a sensitive aptasensor, but provides proof of concept data of the design. Steric and electronic structure activity relationships are discussed for future designs on MG-stilbene conjugates. 
<smiles>CN=C1C=CC(=C(c2ccccc2)c2ccc(N(C)C)cc2)C=C1</smiles>

(1)

Malachite green (MG)

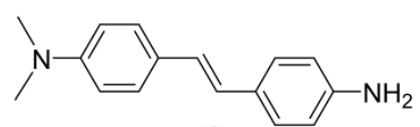

(9)<smiles>CCN(CC)c1ccc2oc(=O)c3ccccc3c3ccc(N(CC)CC)cc3oc2c1</smiles>

(10)

Rhodamine B $(\mathrm{RhB})$<smiles></smiles>

RhB-stilbene-N- $\left(\mathrm{CH}_{3}\right)_{2}$

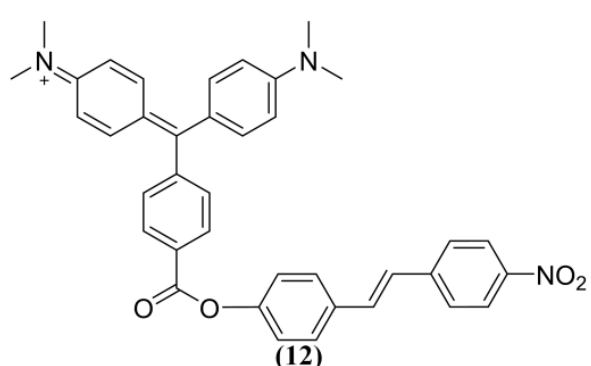

MG-COO-stilbene- $\mathrm{NO}_{2}$

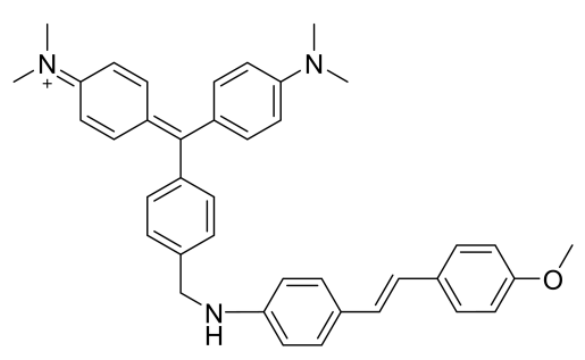

(2)

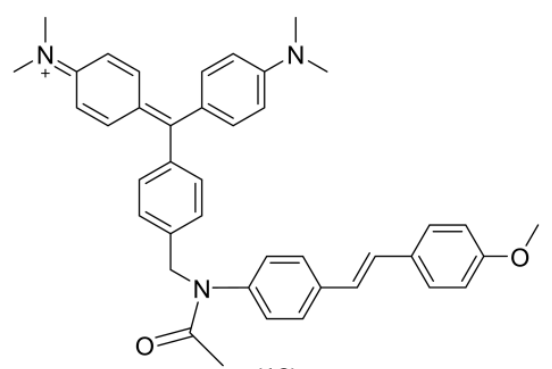

(13)

MG-CH $2-\mathrm{NH}$-stilbene-OMe

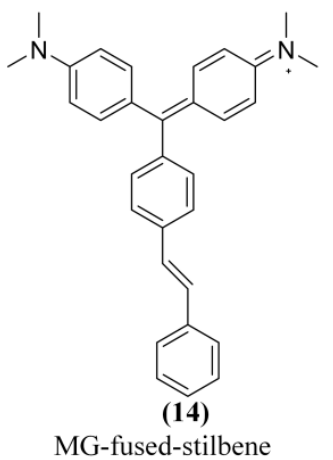

MG-CH $2-\mathrm{NAc}$-stilbene-OMe

MG-fused-stilbene

Figure 5.1 Chemical structures of malachite green (MG) (1), 4-Amino-4'-(N,Ndimethylamino)stilbene $\left(\left(\mathrm{CH}_{3}\right)_{2}-\mathrm{N}\right.$-stilbene- $\left.\mathrm{NH}_{2}\right)(\mathbf{9})$, rhodamine $\mathrm{B}(\mathrm{RhB})(\mathbf{1 0})$ and $\mathrm{MG} / \mathrm{RhB}-$ stilbene conjugates: RhB-stilbene-N-( $\left.\mathrm{CH}_{3}\right)_{2}$ (11), MG-COO-stilbene- $\mathrm{NO}_{2}$ (12), $\mathrm{MG}-\mathrm{CH}_{2}-\mathrm{NH}-$ stilbene-OMe (2), MG-CH $2-\mathrm{NAc}-$ stilbene-OMe (13) and MG-fused stilbene (14).

\subsection{Materials and Methods}

\subsubsection{Materials}

Malachite

green

aptamer

$\left(5^{\prime}-\right.$

GGAUCCCGACUGGCGAGAGCCAGGUAACGAAUGGAUCC-3') (MGA) used in this study was purchased from AITbiotech Pte Ltd Singapore. 4-Amino-4'-(N,Ndimethylamino)stilbene $\left(\left(\mathrm{CH}_{3}\right)_{2}-\mathrm{N}\right.$-stilbene- $\mathrm{NH}_{2}$, trans-isomer $)$, rhodamine $\mathrm{B}(\mathrm{RhB})$ and malachite green oxalate (MG) were purchased from Sigma Aldrich, Singapore. Dimethyl 
$\underline{\text { Binding and Fluorescence Properties of Malachite Green-Stilbene Conjugates Chapter } 5}$

sulfoxide (DMSO) was purchased from Alfa Aesar, USA. HPLC grade acetonitrile was purchased from Tedia, USA. Binding buffer $(\mathrm{pH}=6.7)$ consisted of $5.65 \mathrm{mM} \mathrm{NaH} \mathrm{PO}_{4}$, $4.35 \mathrm{mM} \mathrm{Na}_{2} \mathrm{HPO}_{4}, 10 \mathrm{mM} \mathrm{MgCl}$ and $1 \mathrm{mM} \mathrm{NaCl}$. 96-well polystyrene microplates were purchased from Corning, USA. All materials were used as received.

\subsubsection{MG/RhB-Stilbene Conjugates}

Stilbenes and their MG-conjugates are in trans isomers as default. RhB-stilbene-N$\left(\mathrm{CH}_{3}\right)_{2}$ was synthesized by the project partner PhD Candidate Ms. Wu Yuanyuan. MGCOO-stilbene- $\mathrm{NO}_{2}$ (12), MG- $\mathrm{CH}_{2}-\mathrm{NH}$-stilbene-OMe (2) (not pure), $\mathrm{MG}-\mathrm{CH}_{2}-\mathrm{NAc}$ stilbene-OMe (13) and MG-fused stilbene (14) were synthesized by our group member Dr. Oleksandr Pokholenko. The synthesized conjugates were characterized by NMR and HPLC [1].

\subsubsection{Binding Tests of MGA to MG, RhB and MG/RhB-Stilbene Conjugates}

Direct binding experiments of MGA to MG or MG-stilbene were conducted with conjugates that retained MG's characteristic fluorescence enhancement, as described in section 3.3.1, section 3.3.2 and section 4.2.2.

MG-stilbene conjugates without fluorescence enhancement were analyzed by competitive binding against MG as described in section 3.3.3. Simply summarizing, the concept of competitive binding is to monitor the fluorescence decrease of MGA/MG complex with increasing competitor (MG-stilbene conjugate).

For binding of MGA with RhB (similar to MG in chemical structure) and RhB-stilbene$\mathrm{N}-\left(\mathrm{CH}_{3}\right)_{2}$ as negative control, fluorescence polarization (FP)/fluorescence anisotropy (FA) was applied as RhB has no fluorescence enhancement. Generally, increasing [MGA] was added to constant $(60 \mathrm{nM})$ of $\mathrm{RhB}$ or $\mathrm{RhB}$-stilbene-N-( $\left(\mathrm{CH}_{3}\right)_{2}$, and the anisotropy value was recorded in an Agilent Cary Eclipse fluorescence spectrophotometer (G9800A), with ex/em $=350 \mathrm{~nm} / 445 \mathrm{~nm}$ (obtained by UV-Vis absorption and emission spectrum 
measurements). RhB with fluorescence emission wavelength of around $580 \mathrm{~nm}$ shifts the wavelength to $445 \mathrm{~nm}$ after conjugation with another dye stilbene, which is speculated to be the result of energy transfer between $\mathrm{RhB}$ and stilbene moiety post conjugation.

\subsubsection{Fluorescence Decay of Stilbene Compounds}

Methods for fluorescence decay measurements and data analysis are described in section 3.4. In this chapter, conjugate photoisomerization was conducted by my project partner $\mathrm{PhD}$ candidate Ms. Wu Yuanyuan, while the first order fitting analysis of the $10 \mathrm{~s}$ fluorescence decay was performed by OriginPro 9.1 as mentioned in section 3.4. Briefly, fluorescence emission intensities of stilbene compounds were monitored and recorded over time in a SHIMADZU RF-5301PC Spectrofluorophotometer under constant excitation in corresponding wavelengths.

\subsection{Results and Discussion}

\subsubsection{Binding of MGA with MG (1) and Fluorescence Decay of $\left(\mathrm{CH}_{3}\right)_{2}$-N-stilbene- $\mathrm{NH}_{2}(9)$}

MGA/MG binding positive control is displayed in Figure 5.2, which shows binding affinity of $K_{\mathrm{d}}=200 \mathrm{nM}$. This provides a reference for the binding of MGA to MGstilbene conjugates. MGA/MG binding affinity is retained in the presence of up to $10 \%$ organic additives (ACN, DMSO or EtOH) [2], which extends its application window. Stilbene requires the addition of organic additives due to the poor water solubility. Fluorescence decay of $\left(\mathrm{CH}_{3}\right)_{2}-\mathrm{N}$-stilbene- $\mathrm{NH}_{2}$ in $5 \%$ DMSO/binding buffer is demonstrated in Figure 5.3. Stilbene photoisomerization dependence on viscosity [3-8], was confirmed (Figure 5.4), indicating the micro-environment variation caused by aptamer-ligand recognition may be transduced by stilbene signaling. MG binding and stilbene fluorescence decay displayed in Figure 5.3 and Figure 5.4 serve as positive controls, and will be used for comparison of MG-stilbene conjugate. 


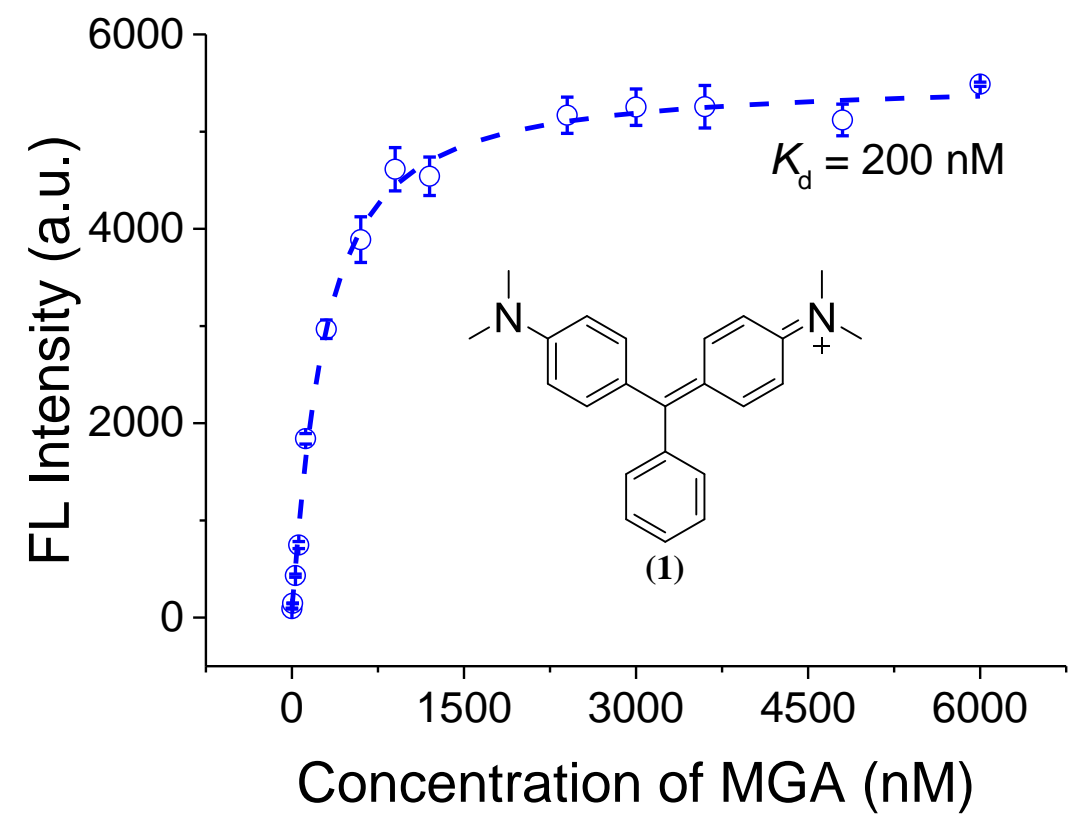

Figure 5.2 MGA/MG binding curve and its calculated $K_{\mathrm{d}}$ based on the fluorescence enhancement of MG (1) at ex/em $=620 \mathrm{~nm} / 656 \mathrm{~nm}$ upon aptamer binding.

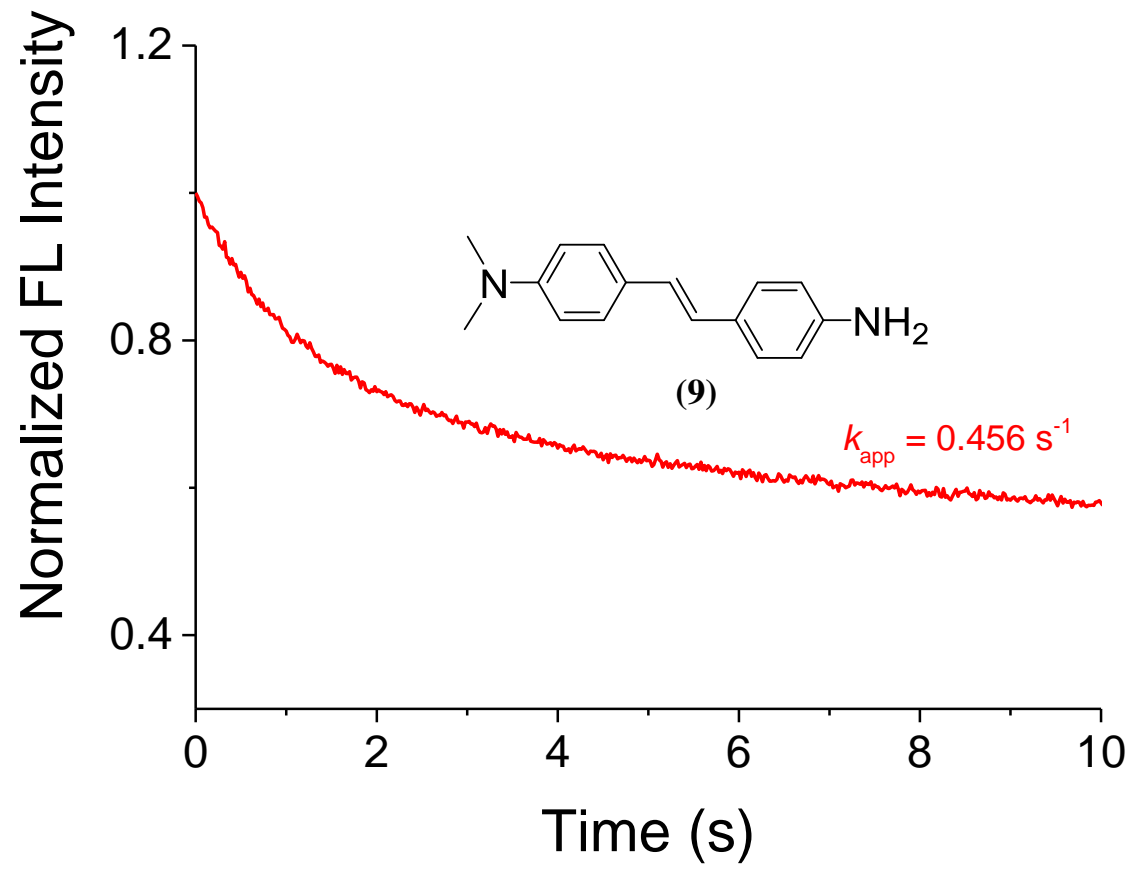

Figure 5.3 Fluorescence decay of $\left(\mathrm{CH}_{3}\right)_{2}-\mathrm{N}$-stilbene- $\mathrm{NH}_{2}(\mathbf{9})$, ex/em $=344 \mathrm{~nm} / 439 \mathrm{~nm}$. 


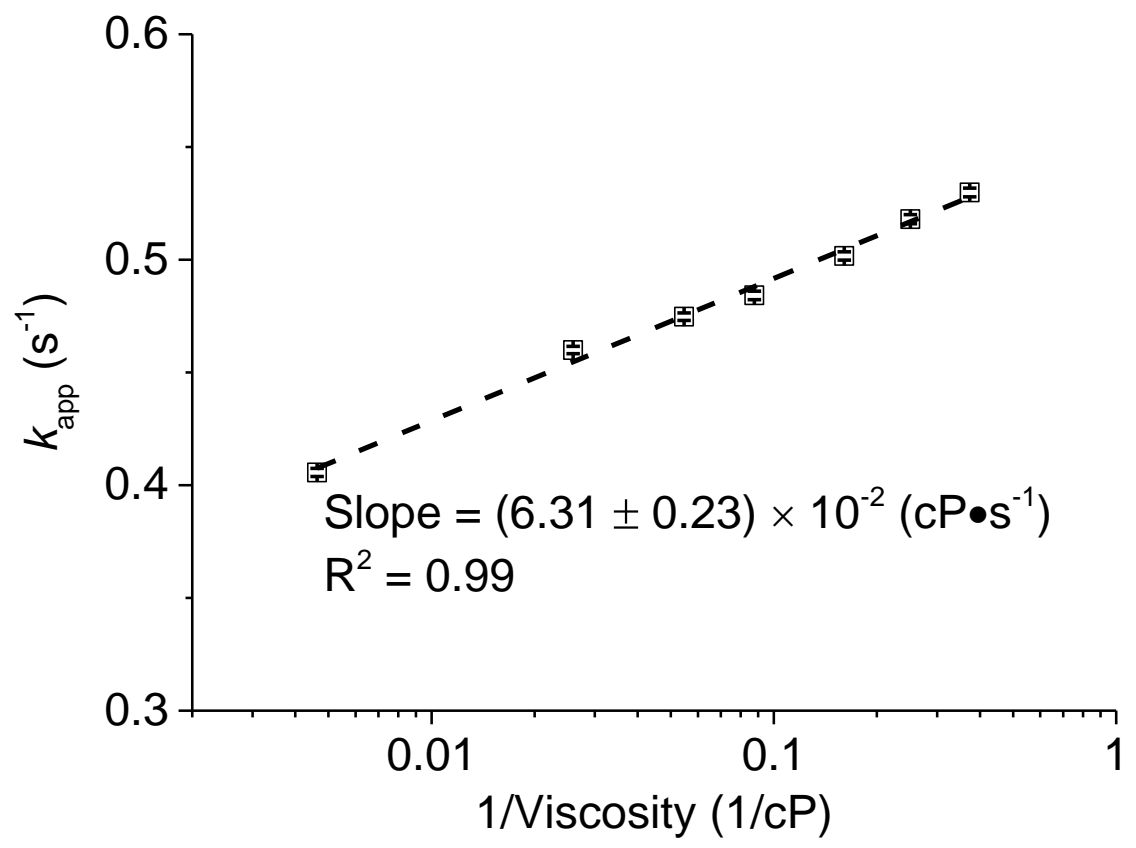

Figure 5.4 Influence of medium viscosity on fluorescence decay rate constant $\left(k_{\text {app }}\right)$ of $\left(\mathrm{CH}_{3}\right)_{2}$-N-stilbene- $\mathrm{NH}_{2}(9)$. Results are from my project partner Ms. Wu Yuanyuan.

\subsubsection{MGA Binding and Fluorescence Decay of RhB-stilbene-N-(CH3) $(11)$}

RhB is chemically similar to MG, but has no binding affinity to MGA. MGA mixed with RhB displays no binding, as seen in Figure 5.5. RhB (10) displays no fluorescence anisotropy variation as [MGA] increases up to $3000 \mathrm{nM}$ [MGA]. Since MGA does not specific bind to $\mathrm{RhB}$, its stilbene conjugate RhB-stilbene-N-( $\left.\mathrm{CH}_{3}\right)_{2}$ can be employed as a negative control to investigate if stilbene conjugates have non-specific binding to MGA. RhB-stilbene-N-( $\left(\mathrm{CH}_{3}\right)_{2}$ photoisomerization is retained, as seen in Figure 5.6. No significant variation in the fluorescence anisotropy of RhB-stilbene-N-( $\left.\mathrm{CH}_{3}\right)_{2}$ is observed with increasing concentration of MGA (Figure 5.7), suggesting no binding interaction between MGA and RhB-stilbene-N-( $\left.\mathrm{CH}_{3}\right)_{2}$. Additionally, the presence of MGA does not interfere the fluorescence decay curves of RhB-stilbene- $\mathrm{N}-\left(\mathrm{CH}_{3}\right)_{2}($ Figure 5.8A\&B$)$, and no significant change in $k_{\text {app }}$ is observed with increasing MGA concentration (Figure 5.9), indicating the fluorescence decay of conjugate will not be affected without specific aptamer binding. Indications are obtained from the results above: 1) fluorescence decay of stilbene in MG-stilbene conjugates is retained, 2) no evidence of MGA non-specific 
binding is observed with stilbene conjugates and 3) no significant difference is seen between the fluorescence decay of stilbene conjugate and its non-binding aptamer mixture. The observation from RhB-stilbene- $\mathrm{N}-\left(\mathrm{CH}_{3}\right)_{2}$ conjugate, presenting no binding with MGA while retaining fluorescence decay, allows us to explore structure activity relationships of MG-stilbene.

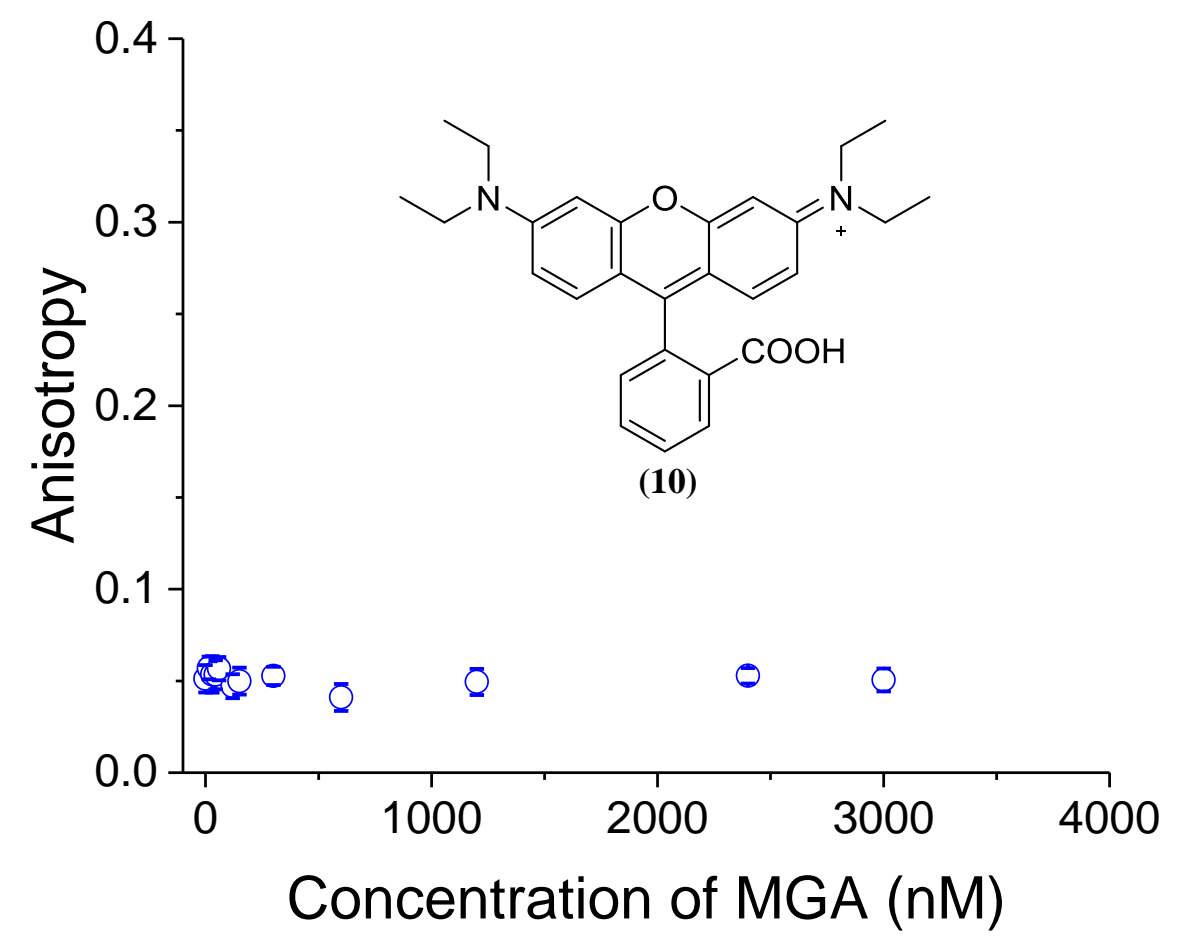

Figure 5.5 Binding of MGA to with RhB (10). No significant change in anisotropy is observed. 


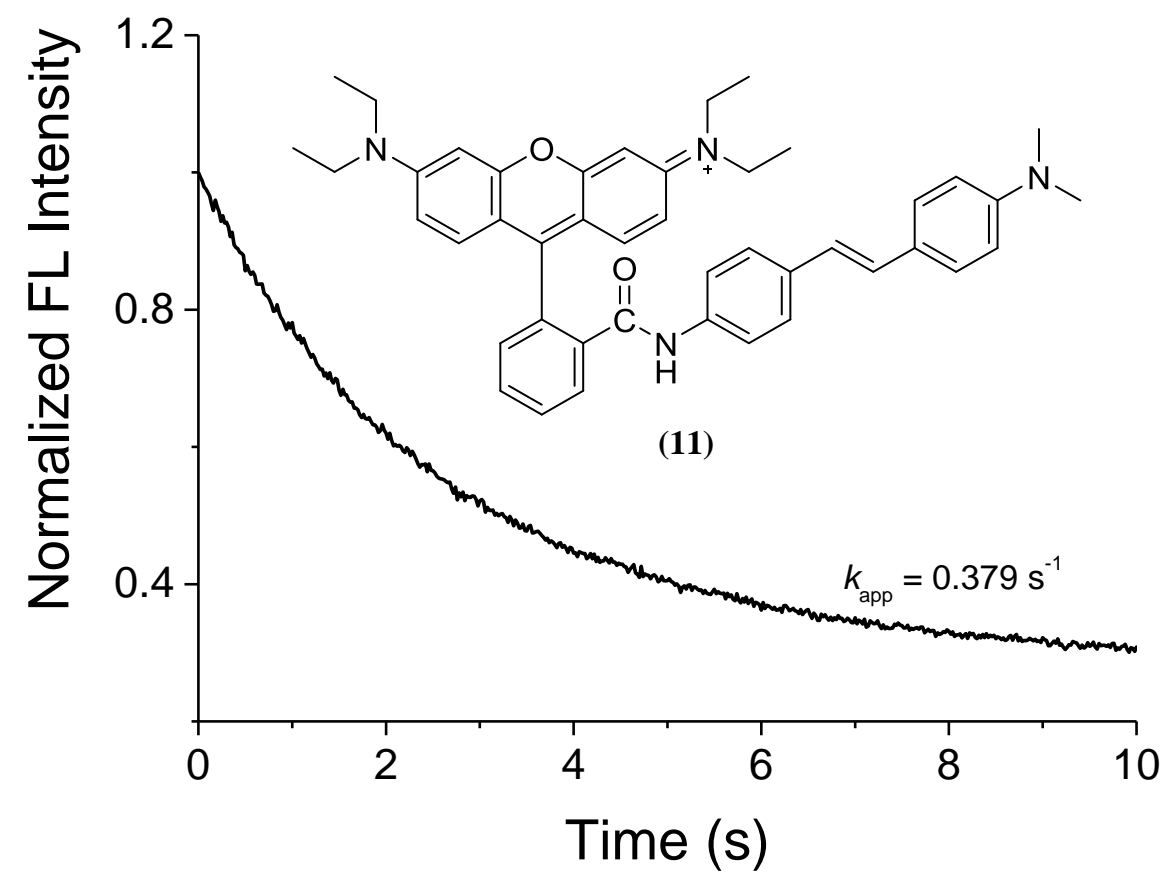

Figure 5.6 Fluorescence decay of RhB-stilbene-N-( $\left(\mathrm{CH}_{3}\right)_{2}(\mathbf{1 1})$, ex/em $=350 \mathrm{~nm} / 445 \mathrm{~nm}$.

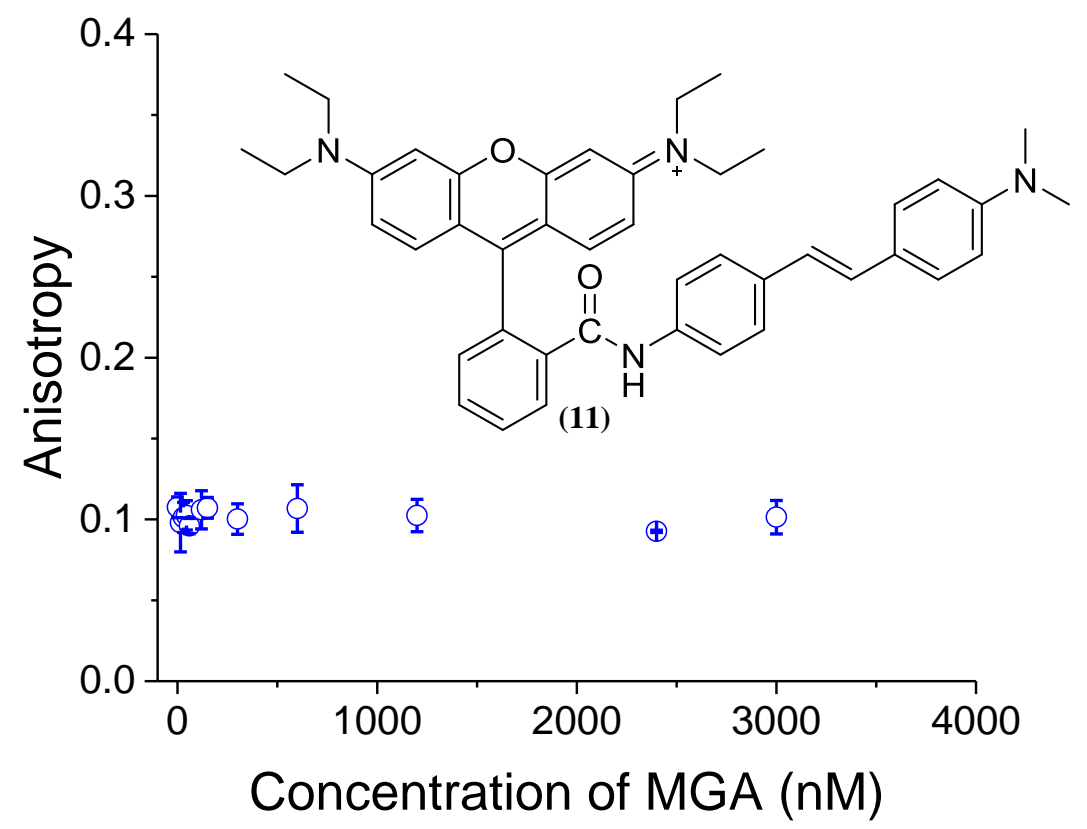

Figure 5.7 Binding of MGA to with RhB-stilbene-N-( $\left(\mathrm{CH}_{3}\right)_{2}$ (11). No significant change in anisotropy is observed. 

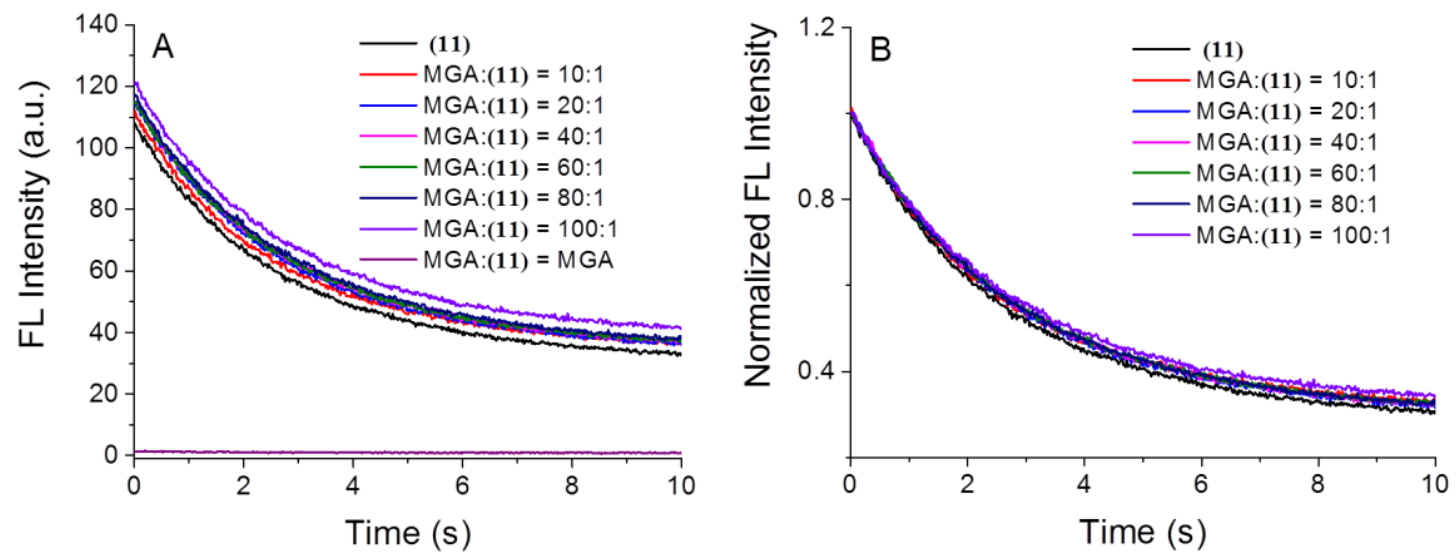

Figure 5.8 (A) Fluorescence decay of RhB-stilbene- $\mathrm{N}-\left(\mathrm{CH}_{3}\right)_{2}(\mathbf{1 1})$ in the presence of different concentration of MGA. (B) Normalized decay curves of A.

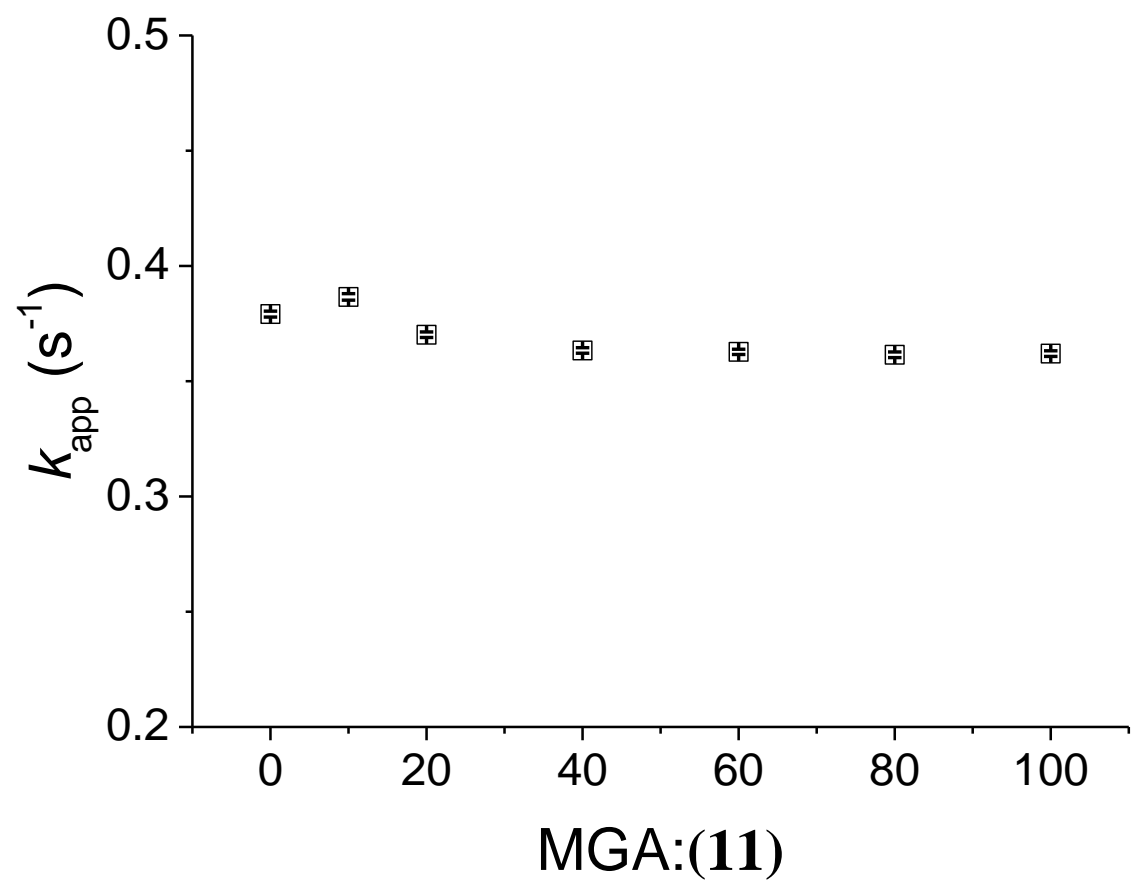

Figure 5.9 $k_{\text {app }}$ of $\mathrm{RhB}$-stilbene- $\mathrm{N}-\left(\mathrm{CH}_{3}\right)_{2}(\mathbf{1 1})$ in the presence of different concentration of MGA.

\subsubsection{MG-COO-stilbene- $\mathrm{NO}_{2}(12)$}

After the control investigations above, MG-COO-stilbene- $\mathrm{NO}_{2}$ conjugate was examined to see if it possesses the required aptamer binding properties before the $-\mathrm{NO}_{2}$ is further 
modified. As preliminary results displayed that the MG moiety had no fluorescence enhancement of this conjugate upon the addition of MGA, competitive binding was applied to investigate its binding affinity with MGA. As seen in Figure 5.10, a $K_{\mathrm{d}}$ up to $6560 \mathrm{nM}$ is obtained. This $K_{\mathrm{d}}$ ( > $6000 \mathrm{nM}$ ) is inefficient and thus further development was arrested for higher priority conjugates. In addition, according to the fluorescence decay experiments, there is no detectable fluorescence decay observed in this conjugate in $10 \%$ DMSO-water, likely due to the $-\mathrm{NO}_{2}$ electron withdrawing group $[9,10]$ (Figure 5.11). MGA/MG binding is mainly attributed to the electrostatic and basic pair stacking/special interaction [11,12]. The weak binding $\left(K_{\mathrm{d}}>6000 \mathrm{nM}\right)$ of MG-COOstilbene- $\mathrm{NO}_{2}$ to MGA may be due to the large molecular volume of the conjugate $(553.92$ $\AA^{3}$ vs. $M G=332.38 \AA^{3}$, calculated by Molinspiration Cheminformatics 2016, the same to all the calculations on molecular volume in this chapter) bothering the base pair stacking, or the strong electron disturbing effect from - $\mathrm{COO}$ - and $-\mathrm{NO}_{2}$ groups affecting the electrostatic interaction, or both. Additionally, the loss of fluorescence decay is probably due to the strong electron accepting effect from $-\mathrm{NO}_{2}$ groups, which has been reported $[9,10]$. Thus, electron donating/accepting effects from linkers/side groups and the size factor of conjugates is further optimized below.

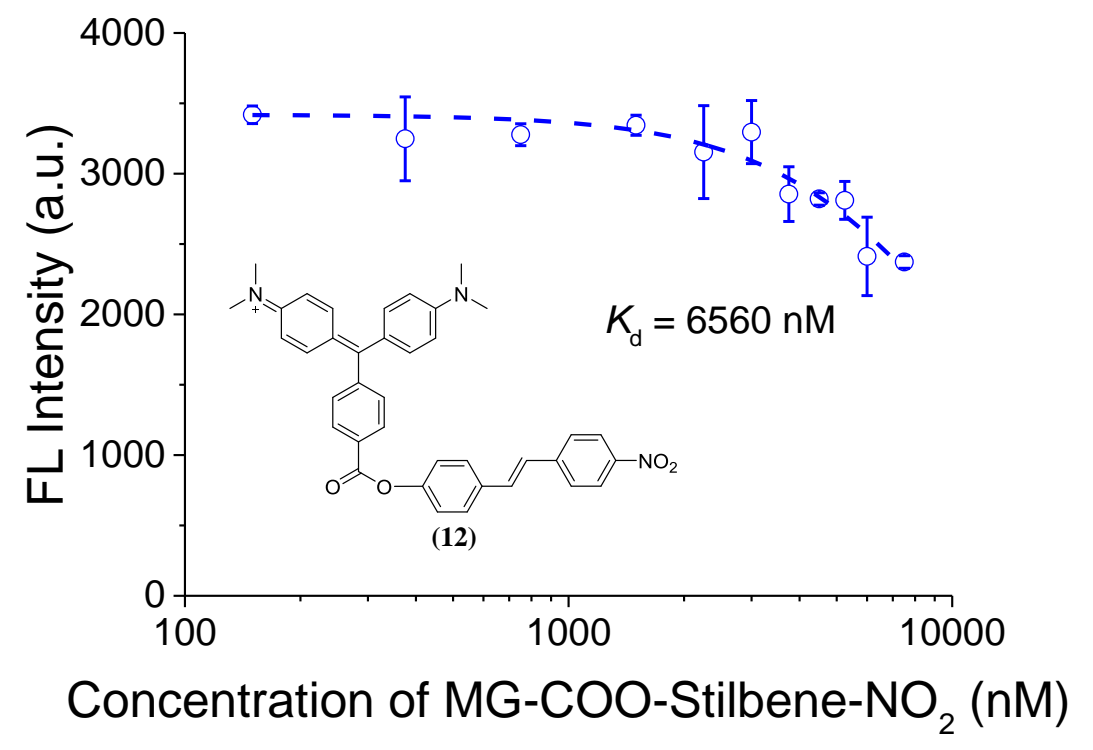

Figure 5.10 Competitive binding of MG-COO-stilbene- $\mathrm{NO}_{2}(\mathbf{1 2})$ to MGA/MG complex and its calculated $K_{\mathrm{d}}$. 


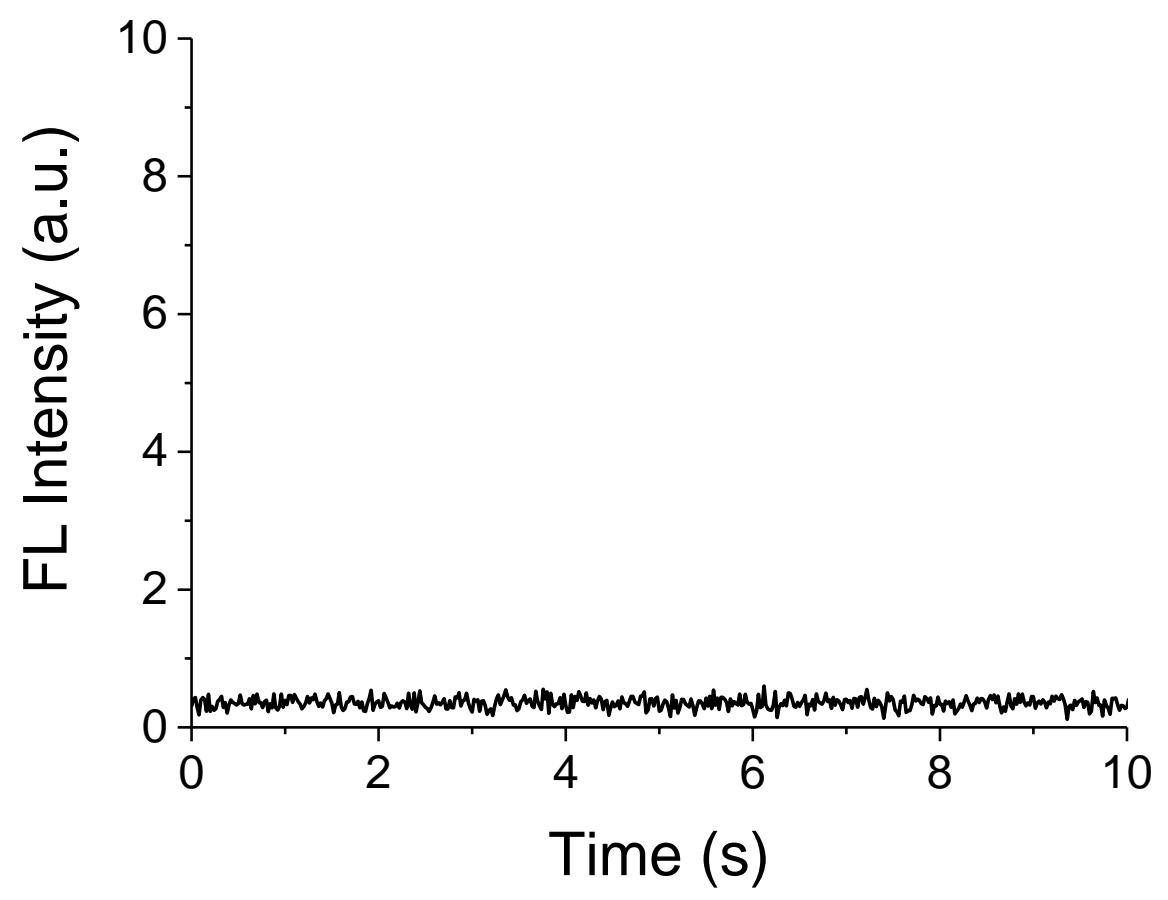

Figure 5.11 Fluorescence decay of MG-COO-stilbene- $\mathrm{NO}_{2}$ (12) at ex/em $=362 \mathrm{~nm} / 520 \mathrm{~nm}$. Results are from my project partner Ms. Wu Yuanyuan.

\subsubsection{MG-CH$-\mathrm{NH}-$-stilbene-OMe (2)}

In order to achieve better binding affinity and fluorescence decay in MG-stilbene conjugate, conjugate MG- $\mathrm{CH}_{2}-\mathrm{NH}$-stilbene-OMe (volume: $557.37 \AA^{3}$, similar to $\mathrm{MG}$ COO-stilbene- $\mathrm{NO}_{2}=553.92 \AA^{3}$ ) was investigated. Different from MG-COO-stilbene$\mathrm{NO}_{2}$, the $-\mathrm{CH}_{2}-\mathrm{NH}$ - linker decouples the electronics of $\mathrm{MG}$ and stilbene. $\mathrm{MG}-\mathrm{CH}_{2}-\mathrm{NH}-$ stilbene-OMe displays no fluorescence enhancement in the presence of MGA, but competitive binding assay shows a $K_{\mathrm{d}}=2380 \mathrm{nM}$ (Figure 5.12). Photoisomerization results in Figure 5.13A\&B confirm significant fluorescence decay in 5\% DMSO/binding buffer $\left(k_{\text {app }}=0.442 \mathrm{~s}^{-1}\right)$. Furthermore, the decay presents even in 2\%-20\% DMSO/binding buffer (Figure 5.13C), which allows hypothesis confirmation under aqueous environments [11,13,14]. $\mathrm{CH}_{2}-\mathrm{NH}$-stilbene-OMe keeps the properties of stilbene fluorescence decay and exhibits measureable binding affinity. Despite the ignorable difference in molecular size as previously described $\left(<10 \AA^{3}\right)$, compared to -COO- and $\mathrm{NO}_{2}$ groups, linkers like $-\mathrm{CH}_{2}-\mathrm{NH}$ - and the stilbene functional group of $-\mathrm{OCH}_{3}$ is beneficial for the MGA binding and stilbene fluorescence decay, respectively. This 
confirms our prediction of electronic decoupling by the $-\mathrm{CH}_{2}$ - linker and electron donating group of $-\mathrm{OCH}_{3}$ on stilbene. However, although the binding affinity of $\mathrm{CH}_{2}$ $\mathrm{NH}$-stilbene-OMe rises ca. 2.8 times compared to MG-COO-stilbene- $\mathrm{NO}_{2}(2380 \mathrm{nM}$ vs. $6560 \mathrm{nM}$ ), it is still low compared to our MG reference ( $2380 \mathrm{nM}$ vs. $200 \mathrm{nM})$. A simple variant, MG- $\mathrm{CH}_{2}-\mathrm{NAc}$-stilbene-OMe, is further tested to improve binding by adding hydrogen acceptors at cost of increasing molecular volume.

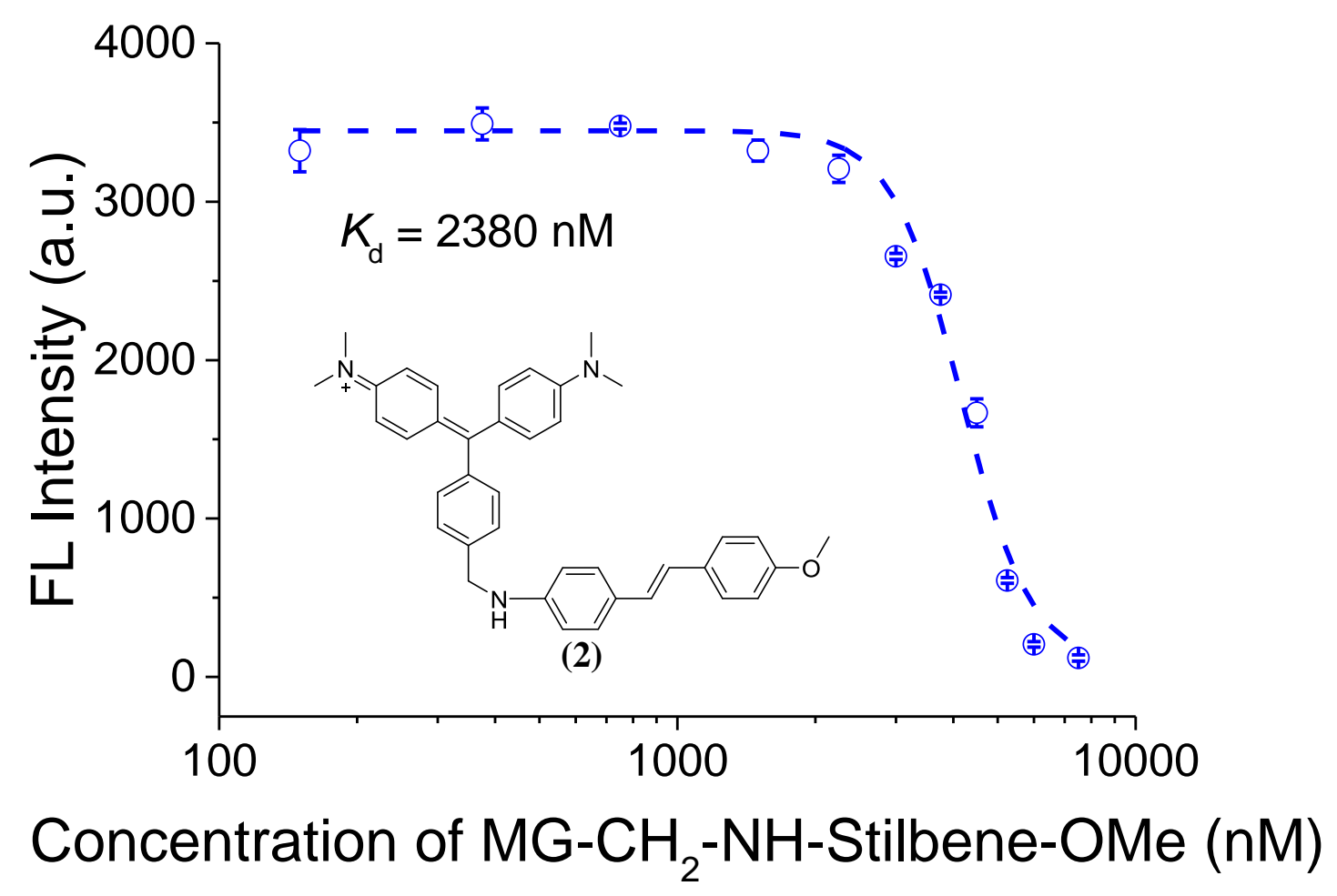

Figure 5.12 Competitive binding of $\mathrm{MG}-\mathrm{CH}_{2}-\mathrm{NH}$-stilbene-OMe (2) to $\mathrm{MGA} / \mathrm{MG}$ complex and its calculated $K_{\mathrm{d}}$. 

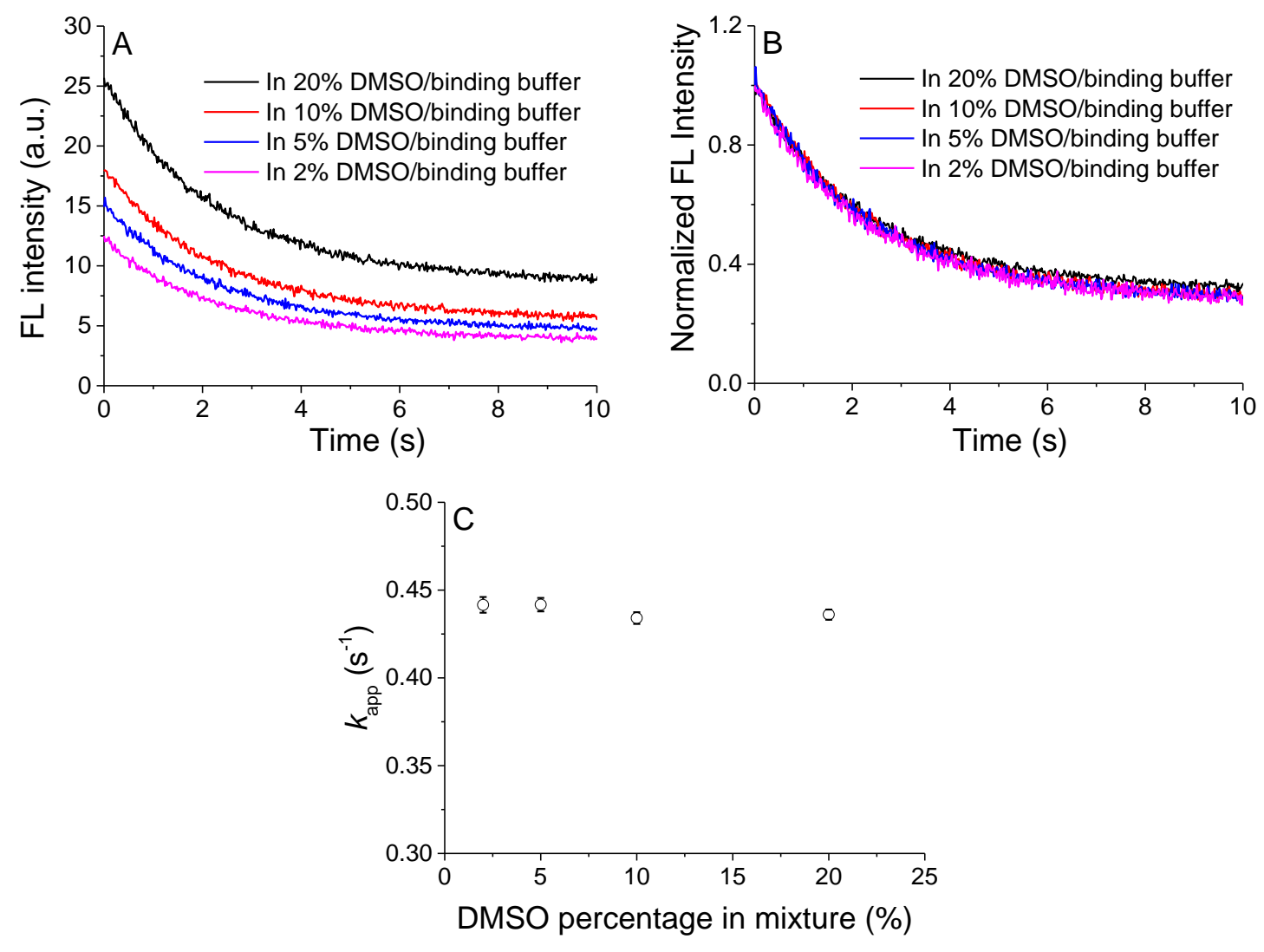

Figure 5.13 (A) Fluorescence decay of $\mathrm{MG}-\mathrm{CH}_{2}-\mathrm{NH}$-stilbene-OMe (2) in different percentage of DMSO/binding buffer at ex/em $=324 \mathrm{~nm} / 432 \mathrm{~nm}$. (B) Normalized fluorescence decay of A. C: Apparent fluorescence decay rate constant $\left(k_{\text {app }}\right)$ of the decay curves. Results are from my project partner Ms. Wu Yuanyuan.

\subsubsection{MG-CH2-NAc-stilbene-OMe (13)}

Regarding to the electrostatic interaction between MGA and MG [11,12], MG- $\mathrm{CH}_{2}-\mathrm{NAc}-$ stilbene-OMe conjugate was tested to see if the $-\mathrm{N}-\mathrm{C}=\mathrm{O}$ linker can enhance the MGA binding by strengthening the hydrogen bonding and electrostatic interaction. The modification has the unexpected benefit of retaining MG's fluorescent enhancement within the MG- $\mathrm{CH}_{2}-\mathrm{NAc}-$ stilbene-OMe conjugate after MGA binding. Direct binding assessment results in a $K_{\mathrm{d}}=1700 \mathrm{nM}$ (Figure 5.14) and the conjugate retained photoisomerisation (Figure 5.15). Similar to $-\mathrm{CH}_{2} \mathrm{NH}-,-\mathrm{CH}_{2}-\mathrm{N}\left(\mathrm{COCH}_{3}\right)$ - linker had fluorescence decay in $5 \% \mathrm{DMSO} /$ binding buffer with $k_{\text {app }}=0.352 \mathrm{~s}^{-1}$, and the decay existed in 2\%-20\% DMSO/binding buffer (Figure 5.15A-C), which makes it possible for 
hypothesis testing. This MG- $\mathrm{CH}_{2}-\mathrm{NAc}-$ stilbene-OMe conjugate has an improved $K_{\mathrm{d}}$ compared to the similar conjugate $\mathrm{MG}-\mathrm{CH}_{2}-\mathrm{NH}$-stilbene-OMe $(1700$ vs. $2380 \mathrm{nM}$, respectively). This was attributed to hydrogen bonding, electrostatic effect, or combination thereof within the $-\mathrm{N}-\mathrm{C}=\mathrm{O}$ functional group, even though the $\mathrm{MG}-\mathrm{CH}_{2}-$ NAc-stilbene-OMe has a larger volume (593.29 $\AA^{3}$ ) compared to MG- $\mathrm{CH}_{2}$-NAc-stilbeneOMe $\left(557.37 \AA^{3}\right)$. This conjugate may be a possible "candidate" for the proof of concept testing but may be limited by sensitivity. Results from this conjugate indicate the introduction of hydrogen bonding and electrostatics with $-\mathrm{N}-\mathrm{C}=\mathrm{O}$ may enhance the MGA binding, but size effect may still be an important factor affecting the aptamer binding. To improve binding, a design to lower molecular volume and retain MG electrostatics was designed into the MG-fused stilbene.

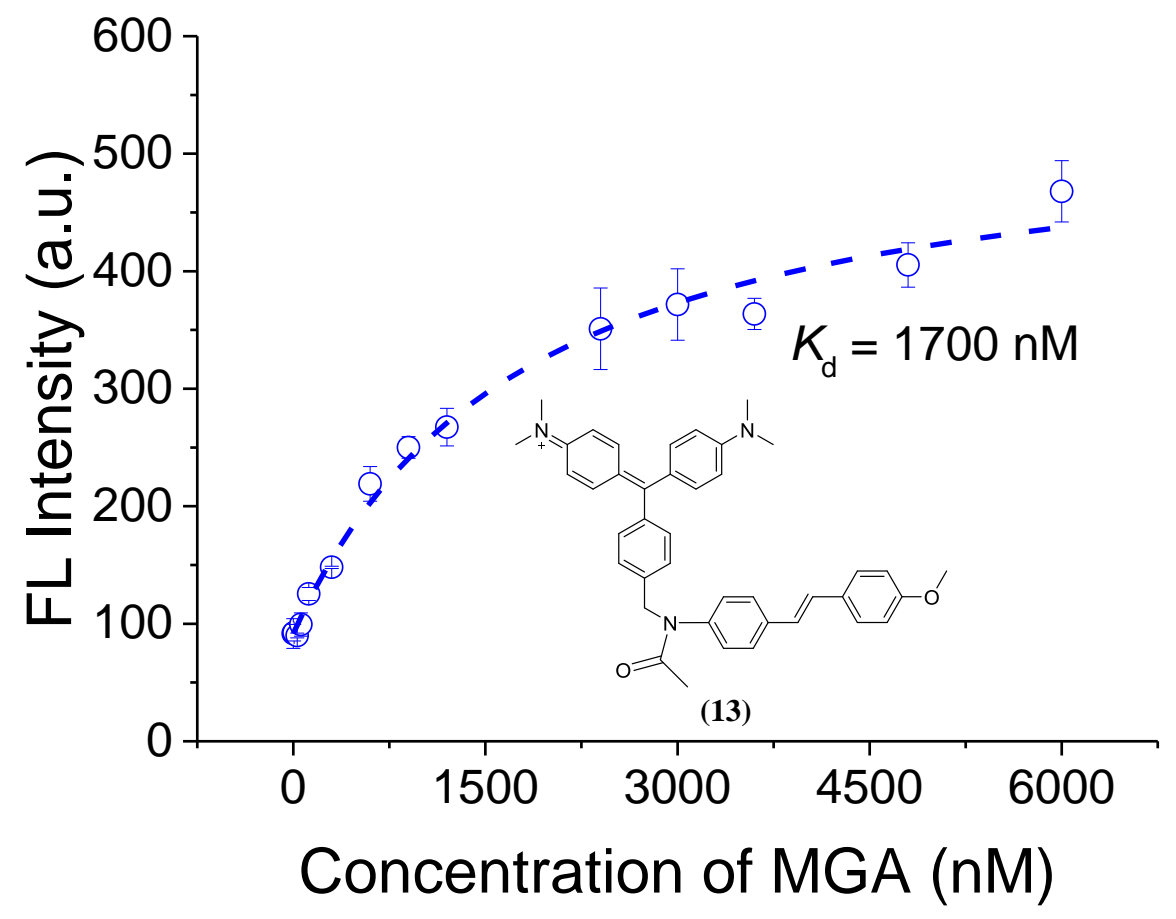

Figure 5.14 Binding of MG-CH$-\mathrm{CAc}$-stilbene-OMe (13) with MGA and its calculated $K_{\mathrm{d}}$ based on fluorescence enhancement. 

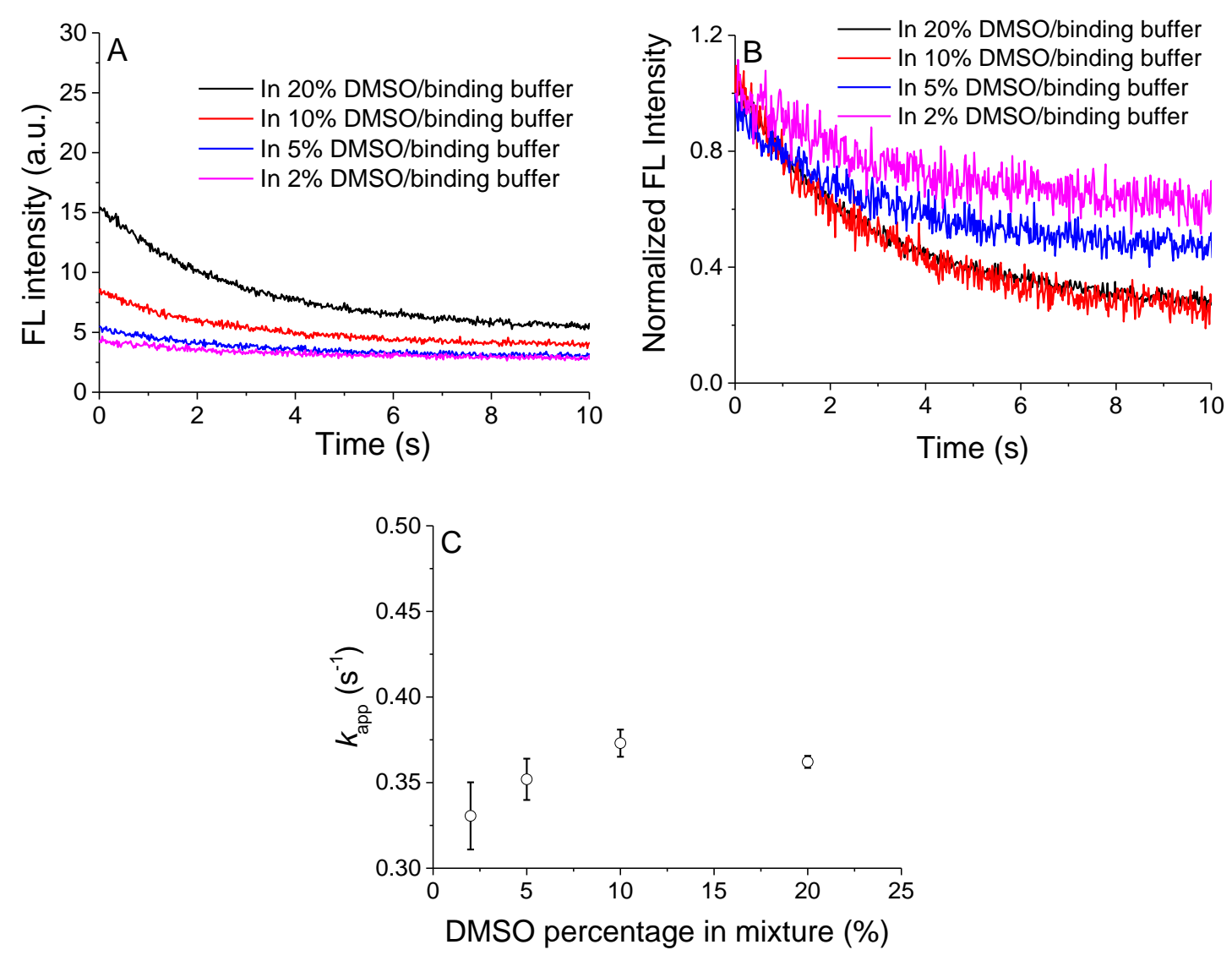

Figure 5.15 (A) Fluorescence decay of $\mathrm{MG}-\mathrm{CH}_{2}-\mathrm{NAc}$-stilbene-OMe (13) in different percentage of DMSO at ex/em $=327 \mathrm{~nm} / 389 \mathrm{~nm}$. (B) Normalized fluorescence decay of A. C: Apparent fluorescence decay rate constant $\left(k_{\text {app }}\right)$ of the decay curves. Results are from my project partner Ms. Wu Yuanyuan.

\subsubsection{MG-fused-stilbene (14)}

Considering the base pair stacking/steric effect on MGA binding [11,12], MG-fusedstilbene with relatively small molecular size may be a good choice for strong aptamer binding. This molecule not only fuses the benzene ring of MG and stilbene, but also removes the side group of stilbene. As a result, this molecule has a size of only $431.21 \AA^{3}$, which is ca. $100 \AA^{3}$ larger than MG, but $100+\AA^{3}$ smaller than all the three MG-stilbene conjugates above. MG-fused-stilbene retains fluorescence enhancement, and the $K_{\mathrm{d}}$ is measured to be $480 \mathrm{nM}$ (Figure 5.16), which is comparable to MG and ideal for our design to investigate the hypothesis in terms of binding affinity. It should be noted that the absolute enhanced fluorescence intensity is only $11 \%$ of MGA/MG binding (Figure 
5.2), which may be due to its less planar structure upon binding $[15,16]$. The lower fluorescence enhancement upon binding suggests the planar interference from the fused stilbene. However, the fluorescence decay of the fused stilbene structure is not retained. Figure 5.17 displays the results of fluorescence decay characterization. Even in pure organic solvents acetonitrile (ACN), dimethyl sulfoxide (DMSO) or ethanol (EtOH), no applicable fluorescence decay is observed. This loss in fluorescence decay may be due to the significant shift in stilbene electronics from fused MG. Thus, this MG-fused-stilbene conjugate cannot be used in the hypothesis testing. However, the results in this conjugate give a critical clue on conjugate design---"Fused" structure with relative small volume (i.e. $<430 \AA^{3}$ ) is good for the retaining of binding affinity, but the loss of fluorescence decay of stilbene needs to be remedied, possibly through the appropriate electron donating or withdrawing group (Figure 5.17).

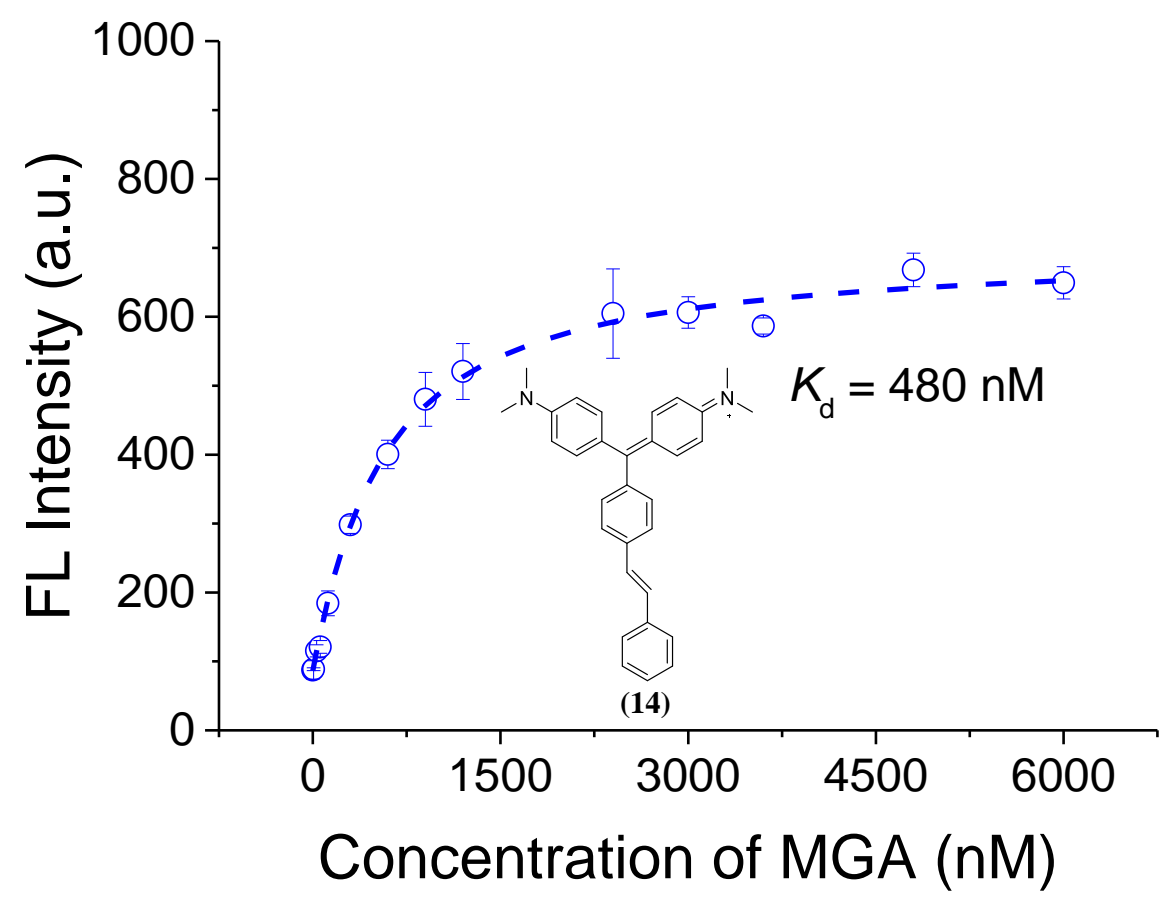

Figure 5.16 Binding of MG-fused-stilbene (14) with MGA and its calculated $K_{\mathrm{d}}$ based on fluorescence enhancement. 


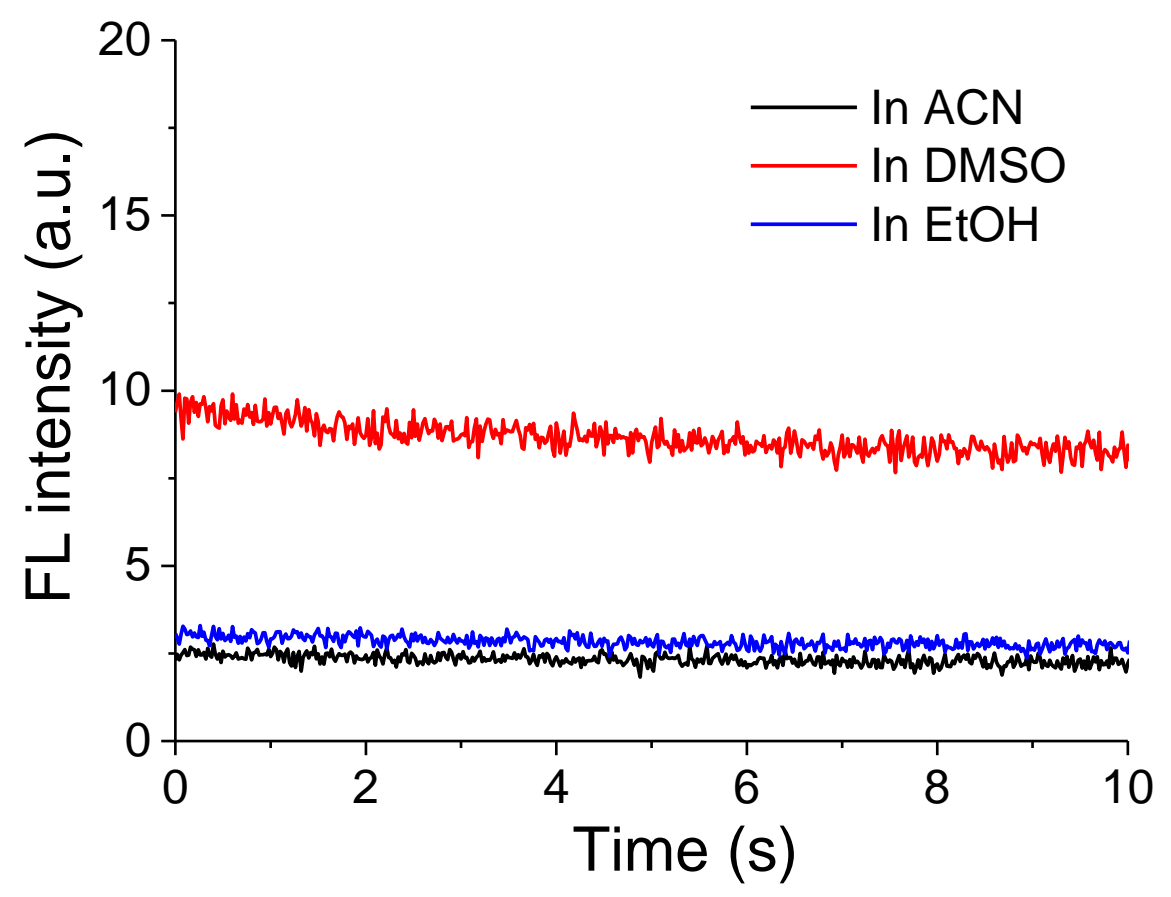

Figure 5.17 Fluorescence decay of MG-fused-stilbene (14) in different organic solvents. at Ex/em = $318 \mathrm{~nm} / 381 \mathrm{~nm}$ for DMSO, $312 \mathrm{~nm} / 398 \mathrm{~nm}$ for ACN and $314 \mathrm{~nm} / 398 \mathrm{~nm}$ for EtOH, respectively. Results are from my project partner Ms. Wu Yuanyuan.

\subsection{Conclusions}

The structure activity relationships of conjugate binding to aptamer and photoisomerization led to conjugates that allow ongoing proof of concept testing. Table 5.1 summarizes the binding and fluorescence decay results. RhB-stilbene- $\mathrm{N}-\left(\mathrm{CH}_{3}\right)_{2}$ as a negative control indicates that 1) no evidence of MGA non-specific binding is observed with stilbene conjugates and 2) no significant difference is seen between the fluorescence decay of stilbene conjugate and its non-binding aptamer mixture. MG-COO-stilbene- $\mathrm{NO}_{2}$ shows negligible binding affinity and fluorescence decay, losing both the properties of MG and stilbene. This observation suggests electron disturbing groups like -COO- should not be linked on $\mathrm{MG}$, and electron accepting groups like $-\mathrm{NO}_{2}$ should not be chosen as side substituent of stilbene as reported [9,10]. Linkers decoupling MG from stilbene should be considered and electron donating groups may be beneficial for stilbene fluorescence decay. $\mathrm{MG}-\mathrm{CH}_{2}-\mathrm{NH}$-stilbene-OMe presents fluorescence decay. The 
outcomes suggest the $-\mathrm{OCH}_{3}$ side group design may be beneficial for retaining the fluorescence decay of stilbene, and the $-\mathrm{CH}_{2}$ - spacer may be helpful for MG binding by decoupling the MG from stilbene, but the low binding affinity ( > $2000 \mathrm{nM}$ ) remains an issue. To improve the binding affinity, factors like size or electrostatic interaction may be considered based on the reported MGA/MG interactions [11,12]. Compared to $\mathrm{MG}-\mathrm{CH}_{2}-$ $\mathrm{NH}$-stilbene-OMe, MG- $\mathrm{CH}_{2}-\mathrm{NAc}-$ stilbene-OMe providing stronger hydrogen bonding and electrostatic interaction brings the $K_{\mathrm{d}}$ down to $1700 \mathrm{nM}$, with similar fluorescence decay kept. The results indicate an approach to enhance the binding affinity by introduction of hydrogen bonding and electrostatics, but size effect may still be an importing factor on binding. MG-fused-stilbene shows a remarkable retaining of binding affinity which is comparable to MG. However, the fluorescence decay of stilbene losses in this conjugate, probably due to the electronic and steric effect from the nearby fused MG, and thus attention is suggested to be focused on retaining the fluorescence decay (i.e. the introduction of the appropriate electron donating group on stilbene) on the design of "fused" conjugates in the future. Some indications on structure activity relationships can be referred from all the results in this chapter. For binding affinity (MG-COO-stilbene$\mathrm{NO}_{2}<\mathrm{MG}-\mathrm{CH}_{2}-\mathrm{NH}$-stilbene-OMe $<\mathrm{MG}-\mathrm{CH}_{2}$-NAc-stilbene-OMe $<$ MG-fused-stilbene $<\mathrm{MG}$ ), small molecular size, suitable decoupling linker for $\mathrm{MG}$, and stronger electrostatic interaction of conjugate may be beneficial for MGA binding. For fluorescence decay, electron donating groups should be preferred while the stilbene should be far from the effect of MG. 
Table 5.1 Summary of the binding and fluorescence results of conjugates and controls.

\begin{tabular}{|c|c|c|c|c|c|c|}
\hline Compound & $\begin{array}{l}\text { Linker/Side } \\
\text { Group }\end{array}$ & $\begin{array}{l}K_{\mathrm{d}} \\
(\mathrm{nM})\end{array}$ & $\begin{array}{l}\text { Method for } K_{\mathrm{d}} \\
\text { Determination }\end{array}$ & $\begin{array}{l}\text { Fluorescence } \\
\text { Decay in } \\
\text { Aqueous } \\
\text { Environment }\end{array}$ & $\begin{array}{l}\text { Molecular } \\
\text { Volume } \\
\left(\AA^{3}\right)^{*}\end{array}$ & Remarks \\
\hline MG (1) & - & 200 & $\begin{array}{l}\text { FI direct } \\
\text { binding }\end{array}$ & - & 332.38 & $\begin{array}{l}\text { Positive } \\
\text { Control }\end{array}$ \\
\hline $\begin{array}{l}(\mathrm{CH} 3)_{2}-\mathrm{N}- \\
\text { stilbene-NH}{ }_{2}(\mathbf{9})\end{array}$ & - & - & - & Yes & - & $\begin{array}{l}\text { Positive } \\
\text { control }\end{array}$ \\
\hline RhB (10) & - & NA & $\begin{array}{l}\text { FP direct } \\
\text { binding }\end{array}$ & - & - & - \\
\hline $\begin{array}{l}\text { RhB-stilbene-N- } \\
(\mathrm{CH} 3)_{2}(\mathbf{1 1})\end{array}$ & $\begin{array}{l}-\mathrm{CO}-\mathrm{NH}-/ \\
-\mathrm{N}\left(\mathrm{CH}_{3}\right)_{2}\end{array}$ & NA & $\begin{array}{l}\text { FP direct } \\
\text { binding }\end{array}$ & Yes & - & $\begin{array}{l}\text { Negative } \\
\text { control }\end{array}$ \\
\hline $\begin{array}{l}\text { MG-COO- } \\
\text { stilbene-NO }{ }_{2}(\mathbf{1 2})\end{array}$ & $\begin{array}{l}-\mathrm{COO}-/ \\
-\mathrm{NO}_{2}\end{array}$ & 6560 & $\begin{array}{l}\text { FI competitive } \\
\text { binding }\end{array}$ & No & 553.92 & $\begin{array}{l}\text { Weakest } \\
\text { binding }\end{array}$ \\
\hline $\begin{array}{l}\text { MG-CH }{ }_{2}-\mathrm{NH}- \\
\text { stilbene-OMe (2) }\end{array}$ & $\begin{array}{l}-\mathrm{CH}_{2}-\mathrm{NH}-/ \\
-\mathrm{OCH}_{3}\end{array}$ & 2380 & $\begin{array}{l}\text { FI competitive } \\
\text { binding }\end{array}$ & Yes & 557.37 & $\begin{array}{l}\text { Weak } \\
\text { binding, } \\
\text { not pure }\end{array}$ \\
\hline $\begin{array}{l}\text { MG-CH} 2-\mathrm{NAc}- \\
\text { stilbene-OMe (13) }\end{array}$ & $\begin{array}{l}-\mathrm{CH}_{2^{-}} \\
\mathrm{N}\left(\mathrm{COCH}_{3}\right)^{-} / \\
-\mathrm{OCH}_{3}\end{array}$ & 1700 & $\begin{array}{l}\text { FI direct } \\
\text { binding }\end{array}$ & Yes & 593.29 & $\begin{array}{l}\text { Weak } \\
\text { binding, } \\
\text { not ideal }\end{array}$ \\
\hline $\begin{array}{l}\text { MG-fused } \\
\text { stilbene (14) }\end{array}$ & - & 480 & $\begin{array}{l}\text { FI direct } \\
\text { binding }\end{array}$ & No & 431.21 & $\begin{array}{l}\text { Strongest } \\
\text { binding }\end{array}$ \\
\hline
\end{tabular}

*Calculated by Molinspiration Cheminformatics 2016: www.molinspiration.com.

NA: no quantitative $K_{\mathrm{d}}$ is available.

-: Not applicable.

\section{References:}

[1] Y. Wu, Nanyang Technological University, 2016.

[2] Y. Zhou; H. Chi; Y. Wu; R. S. Marks; T. W. J. Steele Talanta 2016, 160, 172.

[3] N. Strashnikova; V. Papper; P. Parkhomyuk; G. I. Likhtenshtein; V. Ratner; R. Marks Journal of Photochemistry and Photobiology A: Chemistry 1999, 122, 133.

[4] A. Parthasarathy; L. S. Kaanumalle; V. Ramamurthy Organic Letters 2007, 9, 5059. 
[5] O. Chen; R. Glaser; G. I. Likhtenshtein Journal of Biochemical and Biophysical Methods 2008, 70, 1073.

[6] E. W. Debler; G. F. Kaufmann; M. M. Meijler; A. Heine; J. M. Mee; G. Pljevaljčić; A. J. Di Bilio; P. G. Schultz; D. P. Millar; K. D. Janda; I. A. Wilson; H. B. Gray; R. A. Lerner Science 2008, 319, 1232.

[7] M. R. Ams; D. Ajami; S. L. Craig; J.-S. Yang; J. Rebek, Jr. Beilstein Journal of Organic Chemistry 2009, 5, 79.

[8] D. Tzeli; G. Theodorakopoulos; I. D. Petsalakis; D. Ajami; J. Rebek Journal of the American Chemical Society 2012, 134, 4346.

[9] H. Le Breton; B. Bennetau; J. F. Létard; R. Lapouyade; W. Rettig Journal of Photochemistry and Photobiology A: Chemistry 1996, 95, 7.

[10] V. Papper; G. I. Likhtenshtein Journal of Photochemistry and Photobiology A: Chemistry 2001, 140, 39.

[11] C. Baugh; D. Grate; C. Wilson J Mol Biol 2000, 301, 117.

[12] J. Flinders; S. C. DeFina; D. M. Brackett; C. Baugh; C. Wilson; T. Dieckmann Chembiochem 2004, 5, 62.

[13] E. Kim; C. Yang; Y. Pak Journal of Chemical Theory and Computation 2012, 8, 4845 .

[14] Y. Wu; L. Liu; S. Zhan; F. Wang; P. Zhou Analyst 2012, 137, 4171.

[15] J. R. Babendure; S. R. Adams; R. Y. Tsien Journal of the American Chemical Society 2003, 125, 14716.

[16] S. L. Stead; H. Ashwin; B. Johnston; A. Dallas; S. A. Kazakov; J. A. Tarbin; M. Sharman; J. Kay; B. J. Keely Anal Chem 2010, 82, 2652. 
Binding and Fluorescence Properties of Malachite Green-Stilbene Conjugates Chapter 5 


\section{Chapter 6}

\section{Malachite Green Aptamer-Stilbene Conjugate}

This chapter presents the hypothesis according to the design "stilbeneaptamer conjugate fluorescence decay correlates to analyte binding”. Firstly, stilbene was grafted on malachite green aptamer (MGA) by click chemistry to fabricate the conjugate, and the properties of both MGA in binding and stilbene in fluorescence decay were retained in the conjugate. Fluorescence decay of the conjugate was inhibited in the presence of ligands malachite green and tetramethylrosamine. MGA's adaptive binding may confine the free isomerization of stilbene, supporting our central hypothesis. Analysis of apparent fluorescence decay rate $\left(k_{\text {app }}\right) v$ s. ligand concentration gives a limit of detection (LOD) of $10.5 \mu \mathrm{M}$ for malachite green and $0.7 \mu \mathrm{M}$ for tetramethylrosamine. The novel aptasensor is capable of quantitative sensing within ten seconds of fluorescence decay. This original assay reveals a new fluorescent aptasensing mode employing fluorescence kinetics, which is reported for the first time. The results presented in this chapter support the hypothesis of this thesis within the scope for "stilbene-aptamer conjugate fluorescence decay correlates to analyte binding", under the limited conditions. 


\subsection{Introduction}

As reviewed in Chapter 2, it has been widely reported that aptamers have unique properties of conformational change or structural switching upon binding, making them versatile molecular recognition elements [1-4]. On the other hand, stilbene fluorophores may be used as kinetic fluorescent probes [5-7], whose photoisomerization can be influenced by the surrounding micro-environment such as steric hindrance or viscosity [8-10]. Thus, "stilbene-aptamer conjugate fluorescence decay correlates to analyte binding" (aptamer-stilbene in short) strategy has been designed according to our hypothesis (section 1.4.2), which takes advantages of both conformational change of aptamer and kinetic fluorescence decay of stilbene.

To test the hypothesis regarding the aptamer-stilbene design, a malachite green aptamer (MGA)-stilbene conjugate has been synthesized as an aptasensor and challenged against known ligands. Results show both the MGA's adaptive binding (with MG) and stilbene's fluorescence decay behavior were retained in the conjugate. In the presence of ligand MG, stilbene's fluorescence decay kinetics decreases, due to the conformational change of MGA upon binding. A ligand with higher affinity, tetramethylrosamine (TMR), was quantitated by the synthesized conjugate and the results confirmed the concept of the kinetic fluorescence decay detection mode according to the hypothesis (Figure 6.1). These results support the hypothesis under our limited experimental conditions. The aptasensor shows a never-before detection mode for MG. 


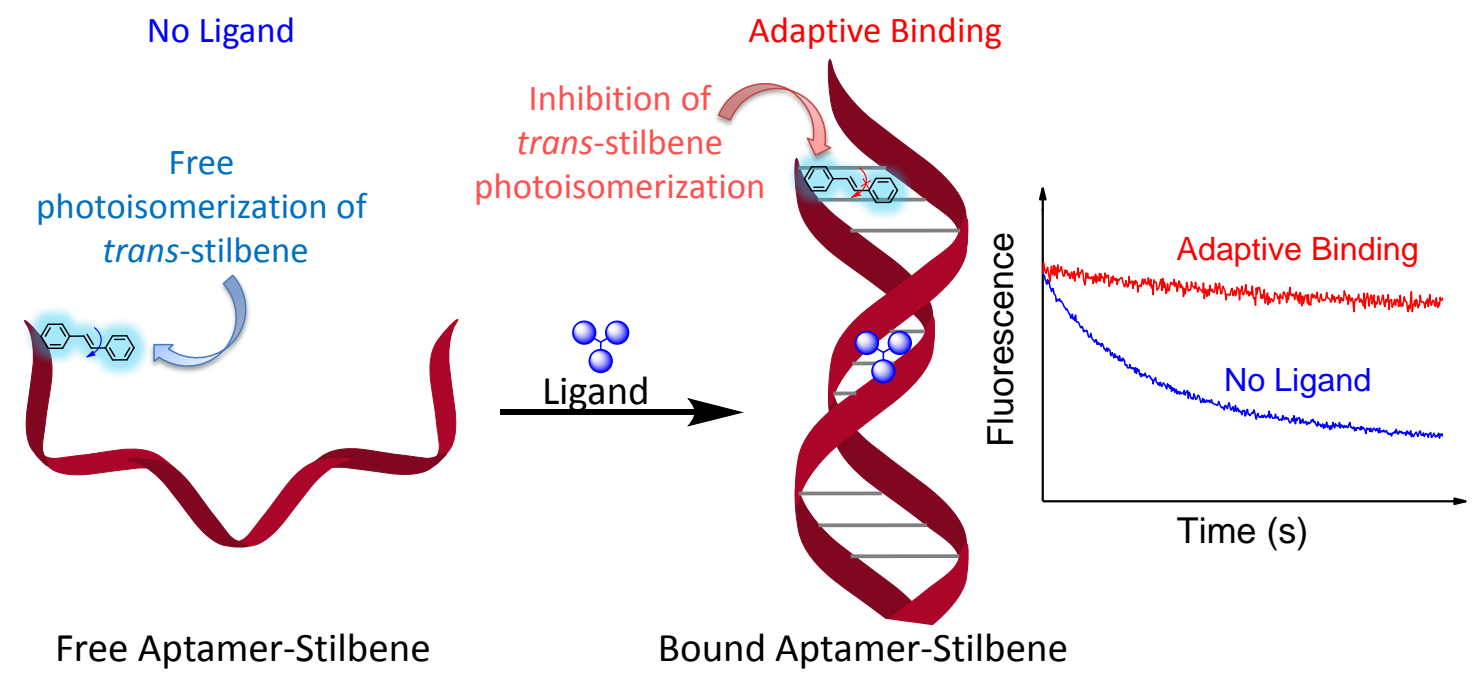

Figure 6.1 Schematic illustration of the aptasensor based on photochrome switch of stilbene and adaptive binding of aptamer. Without analyte (ligand), trans-stilbene grafted on aptamer undergoes free photoisomerization (fluorescent trans form to non-fluorescent cis form), resulting in rapid fluorescence decay. Upon analyte binding, conformational change of aptamer induces an inhibition of the stilbene photoisomerization, resulting in decrease in fluorescence decay kinetics, which can be utilized for biosensing.

\subsection{Materials and Methods}

\subsubsection{Materials}

Malachite

green

aptamer

$\left(5^{\prime}-\right.$

GGAUCCCGACUGGCGAGAGCCAGGUAACGAAUGGAUCC-3') (MGA) and its 3'amino-modified variant (C38) $\mathrm{MGA}-\mathrm{NH}_{2}$ used in this study were purchased from AITbiotech Pte Ltd Singapore. Aminocaproic acid (EACA) (16), lysine (18), 4Acetamido-4'-isothiocyanato-2,2'-stilbenedisulfonic acid disodium salt (SITS) (4), rhodamine B (RhB) (10), malachite green oxalate (MG) (1) were purchased from Sigma Aldrich, Singapore. Tetramethylrosamine (TMR) (15) was purchased from Life Technologies, Singapore. Figure 6.2 displays the structures of MGA, MG, SITS, RhB, TMR, EACA and lysine. Binding buffer $(\mathrm{pH}=6.7)$ consisted of $5.65 \mathrm{mM} \mathrm{NaH}_{2} \mathrm{PO}_{4}, 4.35$ $\mathrm{mM} \mathrm{Na} \mathrm{HPO}_{4}, 10 \mathrm{mM} \mathrm{MgCl} 2$ and $1 \mathrm{mM} \mathrm{NaCl}$ unless specified elsewhere. Amicon 
Centrifugal Filters with $10 \mathrm{kDa}$ molecular weight cut-off were purchased from Merck, Singapore. 96-well polystyrene microplates were purchased from Corning, USA. All materials were used as received. Stilbenes used in decay studies refer to the trans-isomers.

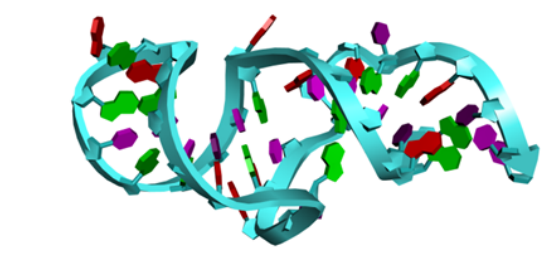

Malachite green aptamer (MGA) 38 nucleotides PubMed ID: 1Q8N

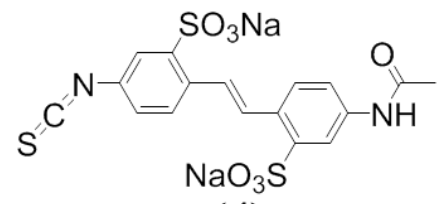

(4)

4-Acetamido-4'-isothiocyanato-2,2'-stilbenedisulfonic acid disodium salt (SITS)

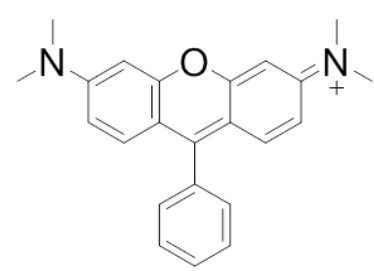

(15)<smiles>NCCCCCC(=O)O</smiles>

(16)

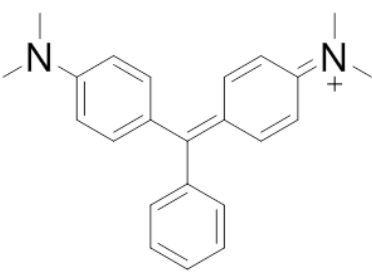

(1)

Malachite green (MG)

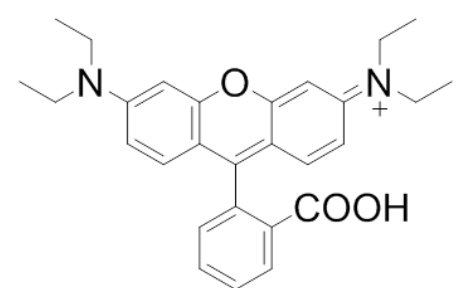

(10)

Rhodamine B (RhB)
Tetramethylrosamine (TMR) Aminocaproic acid (EACA)<smiles>NCCCCC(N)C(=O)O</smiles>

(18)

Figure 6.2 Structures of malachite green aptamer (MGA), malachite green (MG) (1), 4Acetamido-4'-isothiocyanato-2,2'-stilbenedisulfonic acid disodium salt (SITS) (4), rhodamine B $(\mathrm{RhB})(\mathbf{1 0})$, tetramethylrosamine (TMR) (15), aminocaproic acid (EACA) (16) and lysine (18). MGA 3D structure (PDB ID: 1Q8N) is presented by Discovery studio 3.5 Visualizer, Accelrys.

\subsubsection{Synthesis of MGA-SITS Conjugate (5)}

Synthesis of MGA-SITS conjugate follows the classical amine-isothiocyanate reaction commonly used in bioconjugation [11] (Figure 6.3). Briefly, $50 \mu \mathrm{M}$ of $\mathrm{MGA}^{-\mathrm{NH}_{2}}$ was mixed with $5 \mathrm{mM}$ of SITS in $\mathrm{pH}=9$ sodium bicarbonate reaction buffer, with magnetic 
stirring at room temperature for 12 hours and the reaction was prevented from light. After reaction, the excess SITS was eliminated by ultrafiltration with Amicon Centrifugal Filter $(10 \mathrm{kDa})$.

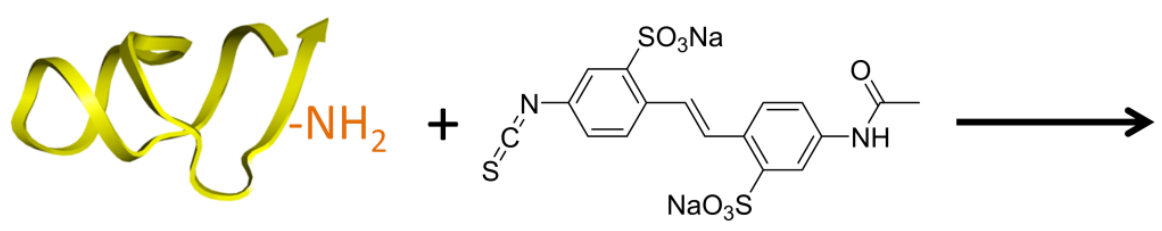

(4)

MGA-NH

SITS

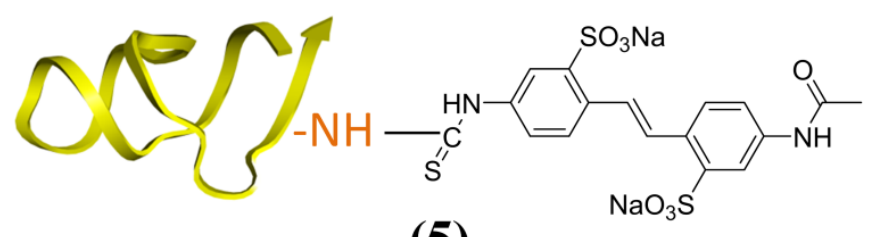

(5)

\section{MGA-SITS conjugate}

Figure 6.3 Synthesis of MGA-SITS conjugate (5).

\subsubsection{Characterization of MGA-SITS by High Performance Liquid Chromatography (HPLC)-Diode Array Detector (DAD)}

An Agilent 1200 series HPLC system with DAD was used for the characterization of MGA-SITS conjugate. Chromatographic separation was conducted using a $5 \mu \mathrm{m} \times 4.6$ $\mathrm{mm} \times 250 \mathrm{~mm}$ Ultisil $^{\mathrm{TM}} \mathrm{C} 18$ column (Welch Materials Inc.), and constant mobile phase that consists of $20 \% 25 \mathrm{mM}$ ammonium acetate $(\mathrm{pH} 6.8)$ and $80 \%$ acetonitrile was applied. The HPLC-DAD system was operated in $1.0 \mathrm{~mL} \cdot \mathrm{min}^{-1}$ flow rate and $10 \mu \mathrm{L}$ sample injection. The DAD was set to $260 \mathrm{~nm}$ and $340 \mathrm{~nm}$ for simultaneously detection of MGA-NH $\mathrm{NH}_{2}$ and SITS respectively, with full spectrum scans from 200 to $800 \mathrm{~nm}$. Herein, HPLC-DAD characterization provided information in both conjugation degree and purity of the synthesized conjugate (removal of free SITS). 


\subsubsection{Matrix-Assisted Laser Desorption/Ionization Time-of-Flight (MALDI-TOF) Mass Spectrum Characterization of MGA-SITS}

Procedures of MALDI-TOF mass spectrum measurement were adapted from literature [12] and the MALDI-TOF experiments were conducted by my group member Dr. Oleksander Pokholenko. Prior to measurement, the samples were desalted with $100 \mathrm{mM}$ ammonium citrate exchange buffer $(\mathrm{pH}=6.0)$ (with 10k Amicon Centrifugal Filters) to suppress the impact from metal cations. After the desalting, $1 \mu \mathrm{L}$ of $10 \mu \mathrm{M}$ sample was mixed with $1 \mu \mathrm{L}$ of THAP matrix $(20 \mathrm{mg} / \mathrm{ml}$ in $4: 1$ mixture of ethanol/aqueous ammonium citrate $(\mathrm{pH}=6 ; 100 \mathrm{mM}))$ on a MALDI-TOF sample plate and the mixture was dried at room temperature [13]. The dried matrix was analyzed in a Shimadzu Axima MALDI-TOF mass spectrometer (in positive linear mode and 1000 profiles).

\subsection{5 $K_{d}$ Determination of MGA-SITS in Binding with MG}

Similar to MGA/MG binding, $K_{\mathrm{d}}$ determination of MGA-SITS binding with MG was measured according to the fluorescence enhancement of MG upon aptamer binding $[14,15]$, which has been discussed in section 3.3. Fluorescence emission spectrum of MG upon MGA-SITS addition was measured with SHIMADZU RF-5301PC Spectrofluorophotometer in "spectrum" mode. In the spectral measurement, mixed solution containing $1 \mu \mathrm{M}$ of MGA-SITS and $1 \mu \mathrm{M}$ of $\mathrm{MG}$ (1:1) was excited at wavelength of $620 \mathrm{~nm}$, with the emission spectrum recorded.

\subsubsection{Measurement of MGA-SITS Fluorescence Decay and Its Aptasensing}

Trans-cis photoisomerization of MGA-SITS conjugate was measured with SHIMADZU RF-5301PC Spectrofluorophotometer with the "time" mode at room temperature, which is described in section 3.4. MGA-SITS samples $(1 \mu \mathrm{M})$ in 5\% DMSO-95\% binding buffer were excited in quartz cuvette at $340 \mathrm{~nm}$, with their fluorescence decays recorded at $\lambda_{\mathrm{em}}=428 \mathrm{~nm}$, while the excitation and emission slits were set to $5 \mathrm{~nm}$. The apparent fluorescence decay rate constant $\left(k_{\text {app }}\right)$ of fluorescence decay for the first 10 second's was 
determined by first order fitting, as described in section 3.4. Sensing of analytes was conducted based on the variation in $k_{\text {app }}$ of MGA-SITS in the presence of various analytes.

\subsubsection{Measurement of SITS Fluorescence Decay in the Presence of MG and MGA/MG Complex}

To investigate the impact of MG and MGA on fluorescence decay of non-conjugated stilbene molecule SITS, fluorescence decay of SITS was monitored in the presence of free MG and MGA/MG (1:20) complex. Same concentrations of SITS and MGA were employed as that used in the MG sensing experiments. Fluorescence decay measurement of SITS (in the presence/absence of MG and MGA) can be referred to section 3.4.

\subsection{Results and Discussion}

A novel aptasensor based on fluorescence decay has been developed, which supports the hypothesis according to the "aptamer-stilbene" design. This aptasensor quantitation mechanism may overcome the existed drawbacks in the traditional "signal on/off" fluorescence sensing mode [16]. Amine MGA and thiol MGA in different position were tested towards binding affinity with MG. The results in Table 6.1 show C38 amine modification presents the lowest $K_{\mathrm{d}}$. Considering the commercially available isothiocyanate stilbene compounds and the ease in large-scale synthesis, C38 aminomodified MGA was chosen for conjugation. MALDI-TOF and HPLC-DAD analyses confirm the successfully synthesis of MGA-SITS conjugate. A quantitative fluorescence decay based sensing of MG is obtained according to the variation of $k_{\text {app }}$. This inhibition effect only appears when the SITS is attached on aptamer, indicated by a negative control experiment with non-conjugated SITS. Additionally, the MGA-SITS sensor has significantly stronger response on TMR (another MGA ligand) compared to MG, while RhB not binding to MGA has no effect on the fluorescence decay of the sensor, showing the selective and multiplex assay of the sensor. 
Table 6.1 MG binding $K_{\mathrm{d}}$ of modified MGA.

\begin{tabular}{llll}
\hline Modified Position & Modification Group & $K_{\mathrm{d}}(\mathrm{nM})$ & $\mathrm{R}^{2}$ \\
\hline C38 & Amine & $200 \pm 30$ & 0.99 \\
C38 & Thiol & $980 \pm 140$ & 0.99 \\
A9 & Thiol & $690 \pm 90$ & 0.98 \\
U4 & Thiol & $820 \pm 90$ & 0.99 \\
C20 & Thiol & $670 \pm 100$ & 0.99 \\
\hline
\end{tabular}

\subsubsection{Successful Synthesis of MGA-SITS Conjugate with Complete Removal of Free SITS}

HPLC-DAD and MALDI-TOF mass spectrum were applied for the characterization of the synthesized MG-SITS. Characterization by HPLC-DAD indicates the successful reaction and removal of free SITS (4) (Figure 6.4) post purification. Under the HPLC conditions in section 6.2.3, $\mathrm{MGA}^{-\mathrm{NH}_{2}}$ peak at retention time (RT) 1.7 min has significant absorbance at $260 \mathrm{~nm}$ but no significant absorbance at $340 \mathrm{~nm}$ (Figure 6.4A), while SITS peak at RT $8.5 \mathrm{~min}$ has absorbance at $340 \mathrm{~nm}$ but no strong absorbance at

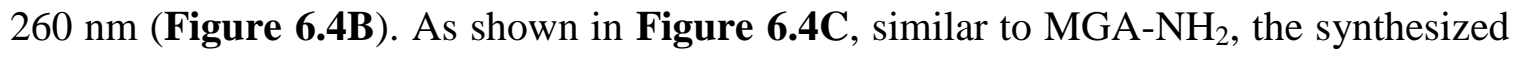
MGA-SITS conjugate peak has a RT of 1.7 min with significant absorbance at $260 \mathrm{~nm}$. Additionally, the peak also presents absorbance at $340 \mathrm{~nm}$, which is the characteristic of SITS but not MGA-NH2 $\left(\mathrm{MGA}-\mathrm{NH}_{2}\right.$ has ignorable UV absorbance at $\left.340 \mathrm{~nm}\right)$. This feature indicates the formation of MGA-SITS conjugate, which should theoretically have UV absorbance at both $260 \mathrm{~nm}$ for $\mathrm{MGA}^{-\mathrm{NH}_{2}}$ and $340 \mathrm{~nm}$ for SITS according to Figure 6.4A and Figure 6.4B. Further information in UV-Vis spectra obtained from the DAD also indicates the achievement of the conjugation, where the UV-Vis spectrum of MGASITS possesses both the feature of $\mathrm{MGA}-\mathrm{NH}_{2}$ and SITS (insets of Figure 6.4A-C). Finally, there is no detectable SITS peak in the chromatography of MGA-SITS, suggesting the complete removal of free SITS in the conjugate (Figure 6.4C). The totally removal of free SITS indicated by Figure 6.4C confirms the fluorescence decay behavior of the synthesized product only belongs to the MGA-SITS conjugate, but not excess SITS. 


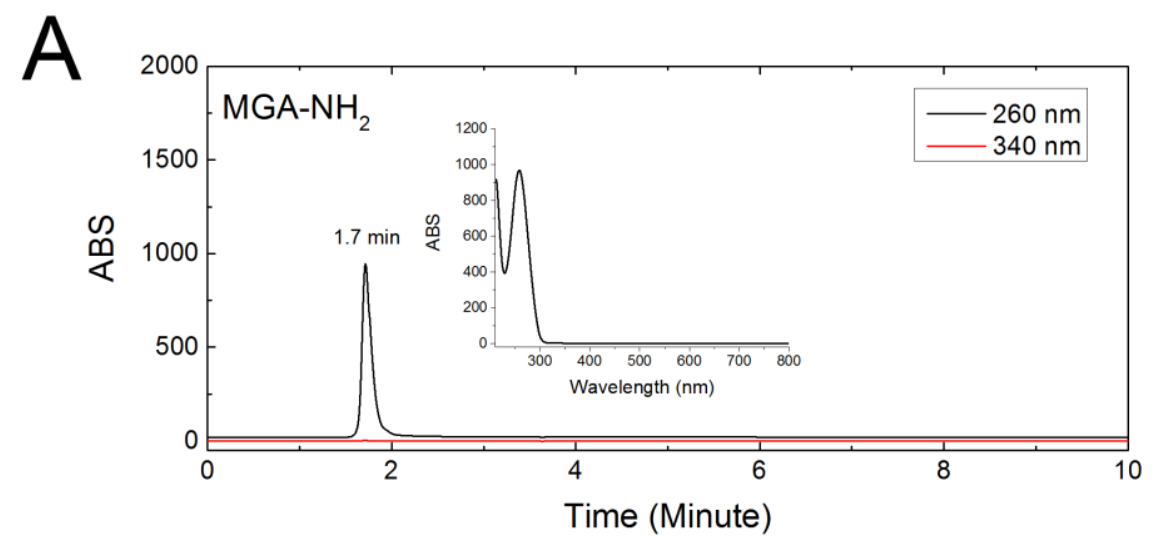

B
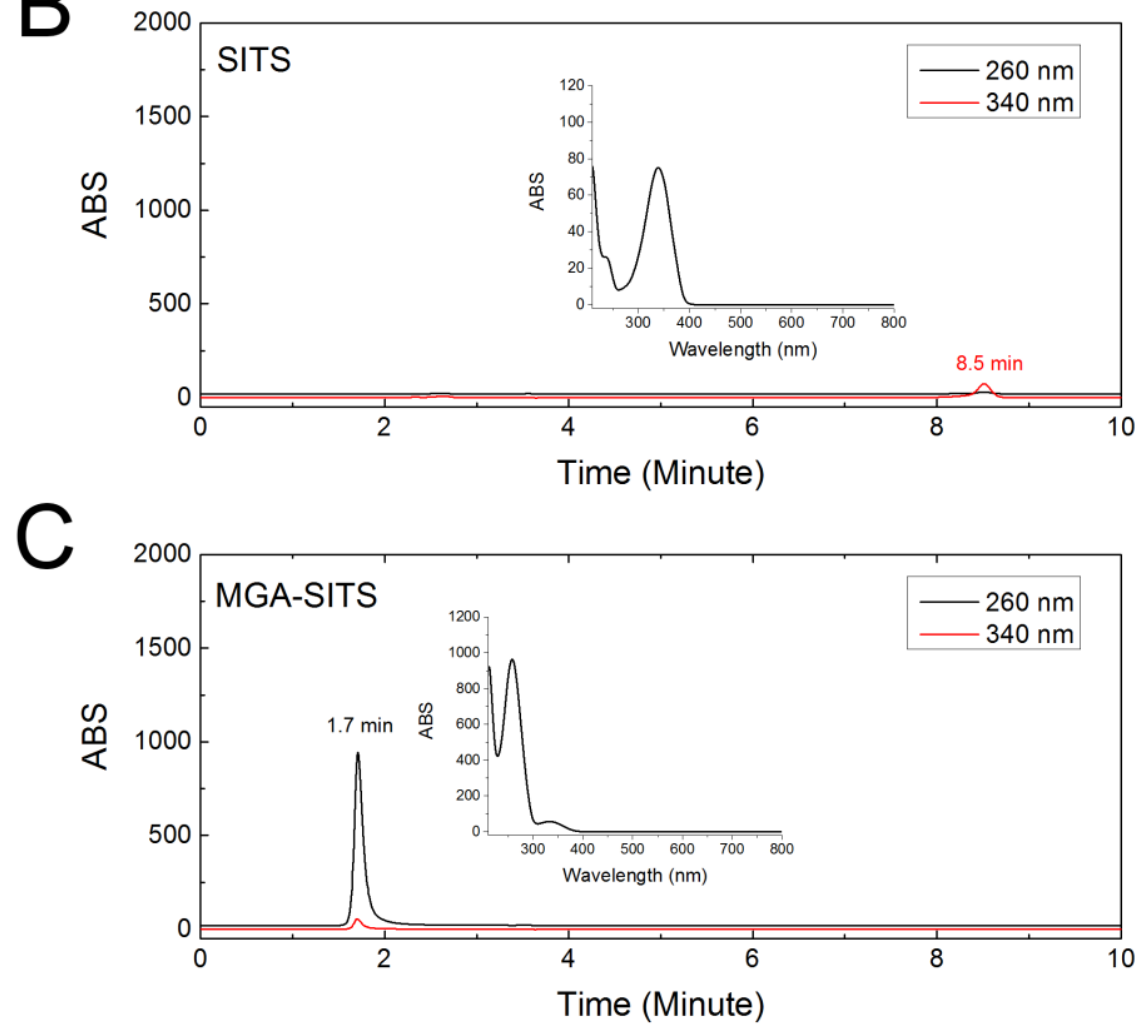

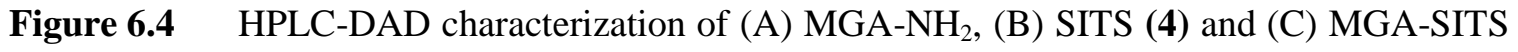
conjugate (5). Insets are the UV-Vis spectra (from DAD) at corresponding RT displaying the variation in absorbance.

As seen in Figure 6.5, starting $\mathrm{MGA}-\mathrm{NH}_{2}$ has $\mathrm{m} / \mathrm{z}$ of $12621[\mathrm{M}+\mathrm{H}]^{+}$(blue). For the spectrum of the product MGA-SITS (red), it possesses m/z of $13072[\mathrm{M}+\mathrm{H}]^{+}$, while the value of initial MGA-NH${ }_{2}$ is $12615[\mathrm{M}+\mathrm{H}]^{+}$within the same spectrum (red). The 457 unit increasing in $\mathrm{m} / \mathrm{z}$ post conjugation indicates the aptamer molecule weight increases 457 
Da due to the attachment of SITS molecule (after replacement of sodium with hydrogen, SITS has a theoretical molecular weight of $454.5 \mathrm{Da}$ ). The small variation in $\mathrm{m} / \mathrm{z}$ value in the results can be considered within instrumental error. The presence of $\mathrm{MGA}-\mathrm{NH}_{2}$ peak in the spectrum of MGA-SITS suggests the remained starting MGA- $\mathrm{NH}_{2}$ in the conjugate or SITS was cleaved during laser ablation, or combination thereof. Assuming the worst case scenario of non-conjugated $\mathrm{MGA}-\mathrm{NH}_{2}$, its presence will not affect the fluorescence decay of the MGA-SITS but may reduce overall sensitivity of the aptasensor. In summary, MALDI-TOF spectra results combining with the HPLC-DAD support the synthesis of MGA-SITS without an excess of free SITS.

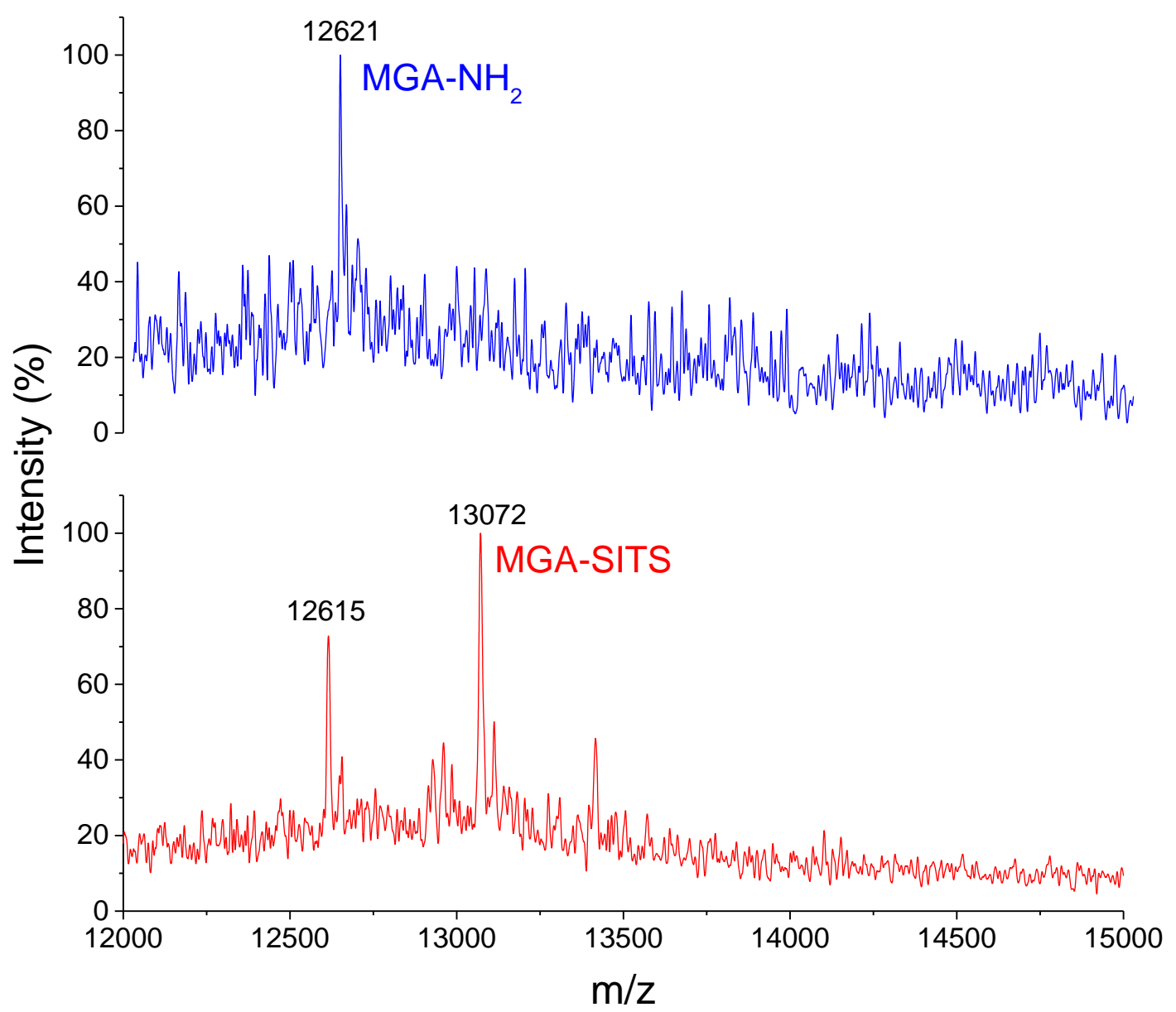

Figure 6.5 MALDI-TOF spectra of $\mathrm{MGA}^{-\mathrm{NH}_{2}}$ (top blue) and MGA-SITS conjugate (5) (bottom red). 


\subsubsection{Optimizations on Bioconjugation Reaction}

Reaction conditions were initially optimized on the reaction of SITS with inexpensive aminocaproic acid (EACA) (16) and lysine (18) (Figure 6.2). HPLC-DAD was employed for monitoring the reaction by tracing the absorbance of SITS. Figure 6.6A displays the EACA-SITS reaction while Figure 6.7A shows the lysine-SITS reaction, at $4 \operatorname{deg} \mathrm{C}$ and room temperature. Less than $50 \%$ of SITS was reacted at $4 \mathrm{deg}$ C (Figure 6.6B) with EACA. At $12 \mathrm{~h}$ at room temperature, reaction was nearly complete (Figure 6.6C). In the reaction with lysine, completion was reached at $24 \mathrm{~h}$ at $4 \mathrm{deg} \mathrm{C}$ (Figure 6.7B), or $4 \mathrm{~h}$ at room temperature (Figure 6.7C). Although lysine reacts faster than EACA with SITS, owing to the single $-\mathrm{NH}_{2}$ group on MGA, EACA reaction should be a more proper reference for the reaction of MGA-NH$H_{2}$ with SITS. Thus, room temperature and at least 8 $\mathrm{h}$ should be chosen for a relatively rapid and complete reaction with amino-modified aptamer.
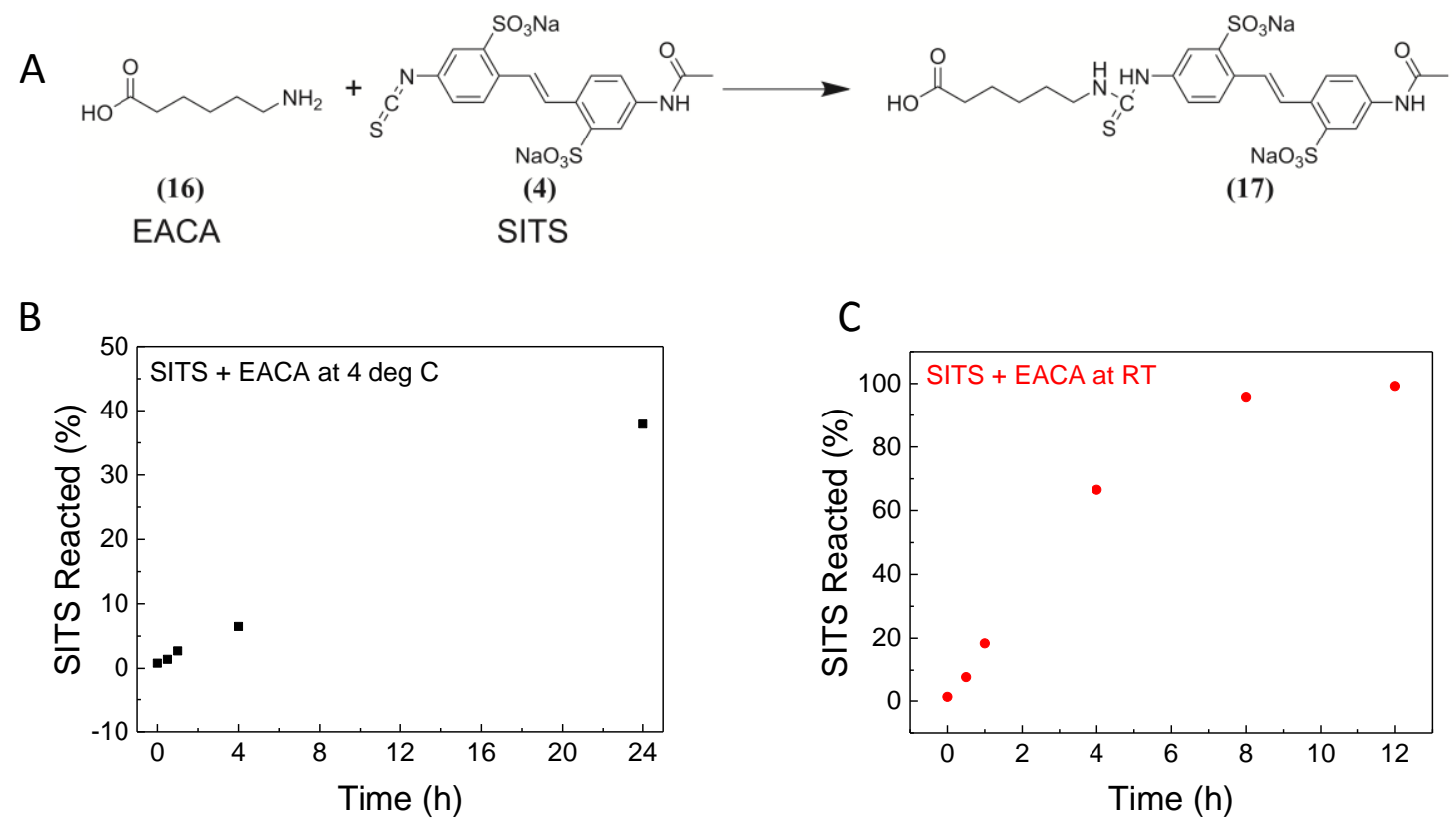

Figure 6.6 (A) Reaction of EACA (16) with SITS (4). (B) Reaction progress at 4 deg C. (C) Reaction progress at room temperature. 


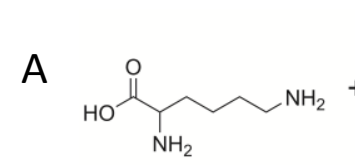

(18) Lysine

B

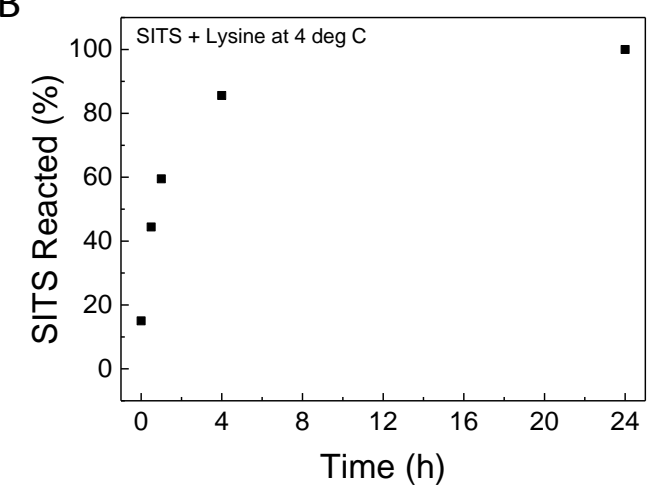

SITS

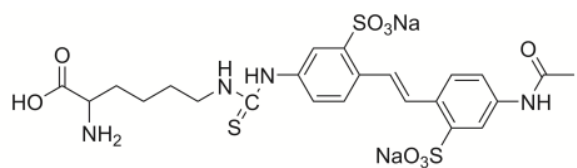

(19)

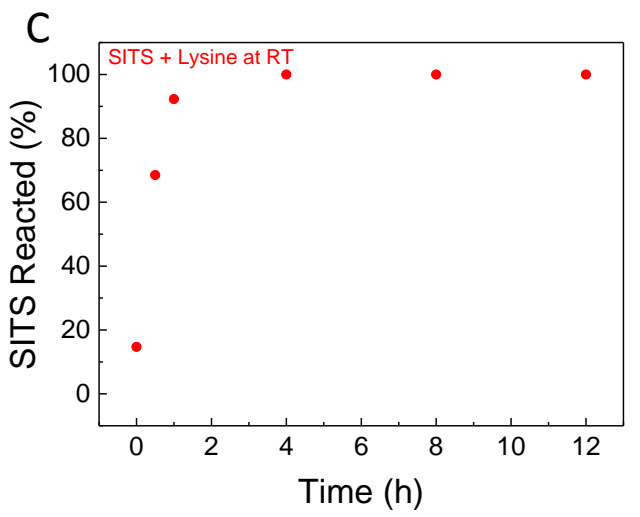

Figure 6.7 (A) Reaction of lysine (17) with SITS (4). (B) Reaction progress at 4 deg C. (C) Reaction progress at room temperature.

The reaction of MGA- $\mathrm{NH}_{2}$ with SITS at room temperature was monitored by HPLCDAD, based on the increase of SITS peak $(\lambda=340 \mathrm{~nm})$ over time on the RT of MGA$\mathrm{NH}_{2}$ peak (Figure 6.8 A). As shown in Figure 6.8 B, the conjugation is nearly complete at $12 \mathrm{~h}$. Although reaction time up to $48 \mathrm{~h}$ is more beneficial for the degree of reaction, considering the stability of RNA aptamer and reaction system, reaction time $12 \mathrm{~h}$ was chosen. 

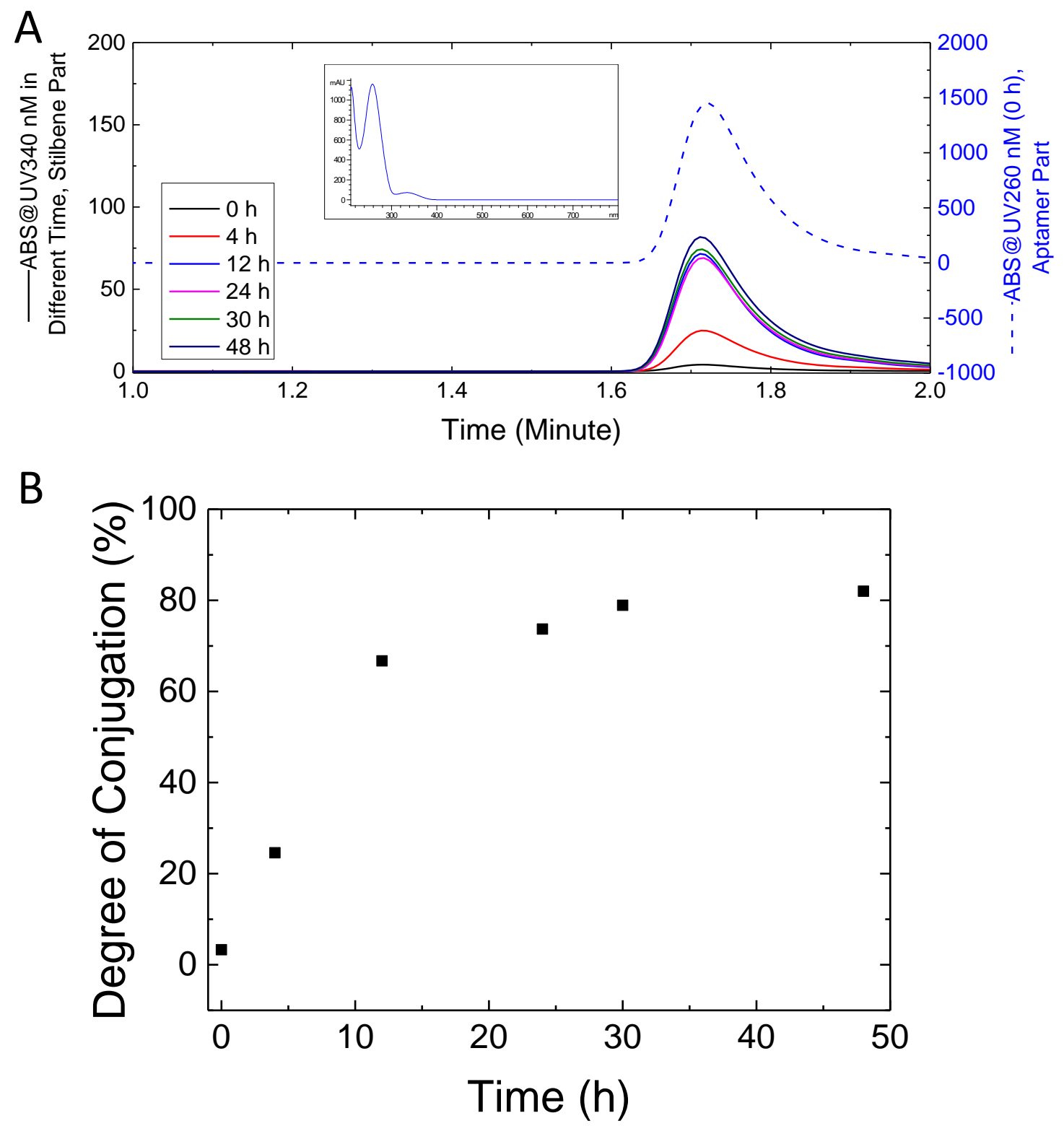

Figure 6.8 (A) HPLC monitoring of absorbance increase in UV340 (grafting of SITS (4)) over time at RT 1.7 min. (B) The calculated degree of conjugation as a function of time.

\subsubsection{MGA-SITS Keeps Properties of Aptamer Binding and Stilbene Fluorescence Decay}

Prior to sensing applications/hypothesis testing, MGA-SITS conjugate needs to be assessed for both the fluorescence decay behavior (property of SITS) and binding to MG 
(property of MGA). Figure 6.9A exhibits the fluorescence decay of MGA-SITS, presenting its retained fluorescence decay property of SITS $\left(k_{\mathrm{app}}: 0.360 \mathrm{~s}^{-1}\right.$ for MGASITS vs. $0.379 \mathrm{~s}^{-1}$ for SITS). Based on the fluorescence enhancement of MG upon aptamer binding, as described in section 3.3.1, the direct binding between MGA-SITS and $\mathrm{MG}$ was confirmed [14,15]. Figure 6.9B displays fluorescence emission (enhancement) of MG at wavelength around $650 \mathrm{~nm}$ upon addition of MGA-SITS, suggesting the binding between MGA-SITS and MG. For the determination of binding affinity, according to the method in section 3.3.2, the $K_{\mathrm{d}}$ of MGA-SITS binding with MG was determined to be $240 \pm 10 \mathrm{nM}$, which compares well to the $K_{\mathrm{d}}$ of $\mathrm{MGA}-\mathrm{NH}_{2}$ (starting material for the synthesis of MG-SITS) binding to MG (200 $\pm 30 \mathrm{nM}$ ), showing no significant difference between each other (Figure 6.10). These results confirm that MGA-SITS keeps the properties of both aptamer in MG binding and stilbene in fluorescence decay, which allows for the hypothesis to be challenged under limited conditions.
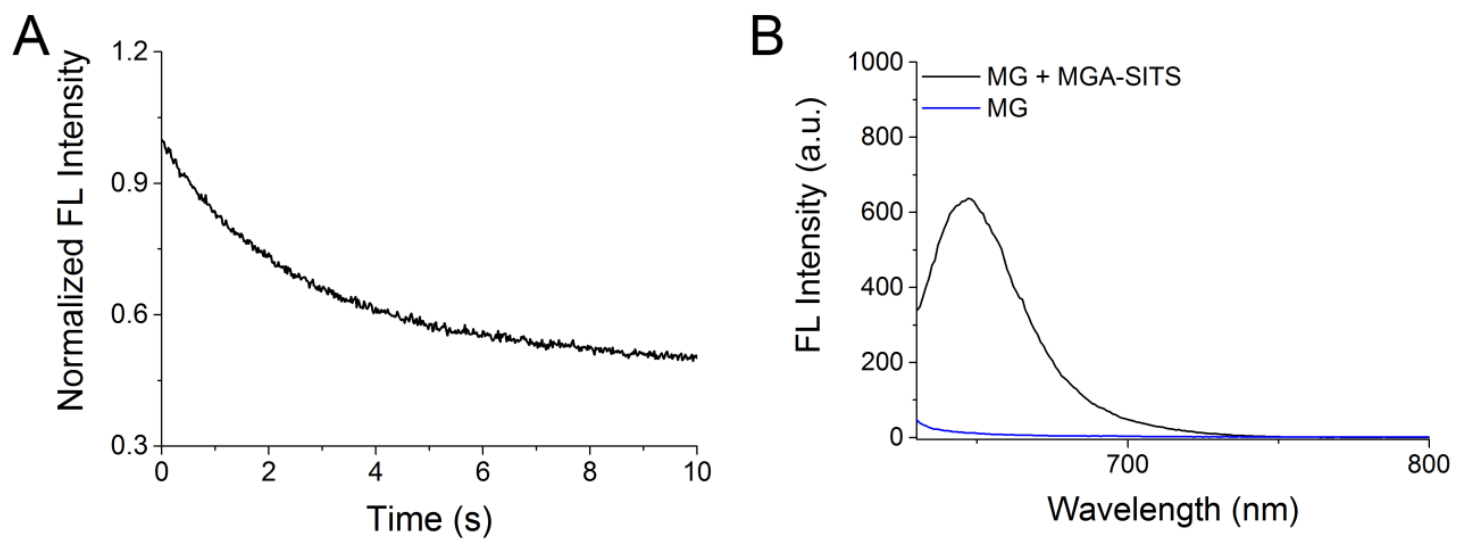

Figure 6.9 (A) Fluorescence decay of MGA-SITS (5) at ex/em $=340 \mathrm{~nm} / 428 \mathrm{~nm}$. (B) Fluorescence emission of $\mathrm{MG}$ in the absence and presence of MGA-SITS at excitation wavelength of $620 \mathrm{~nm}$. 


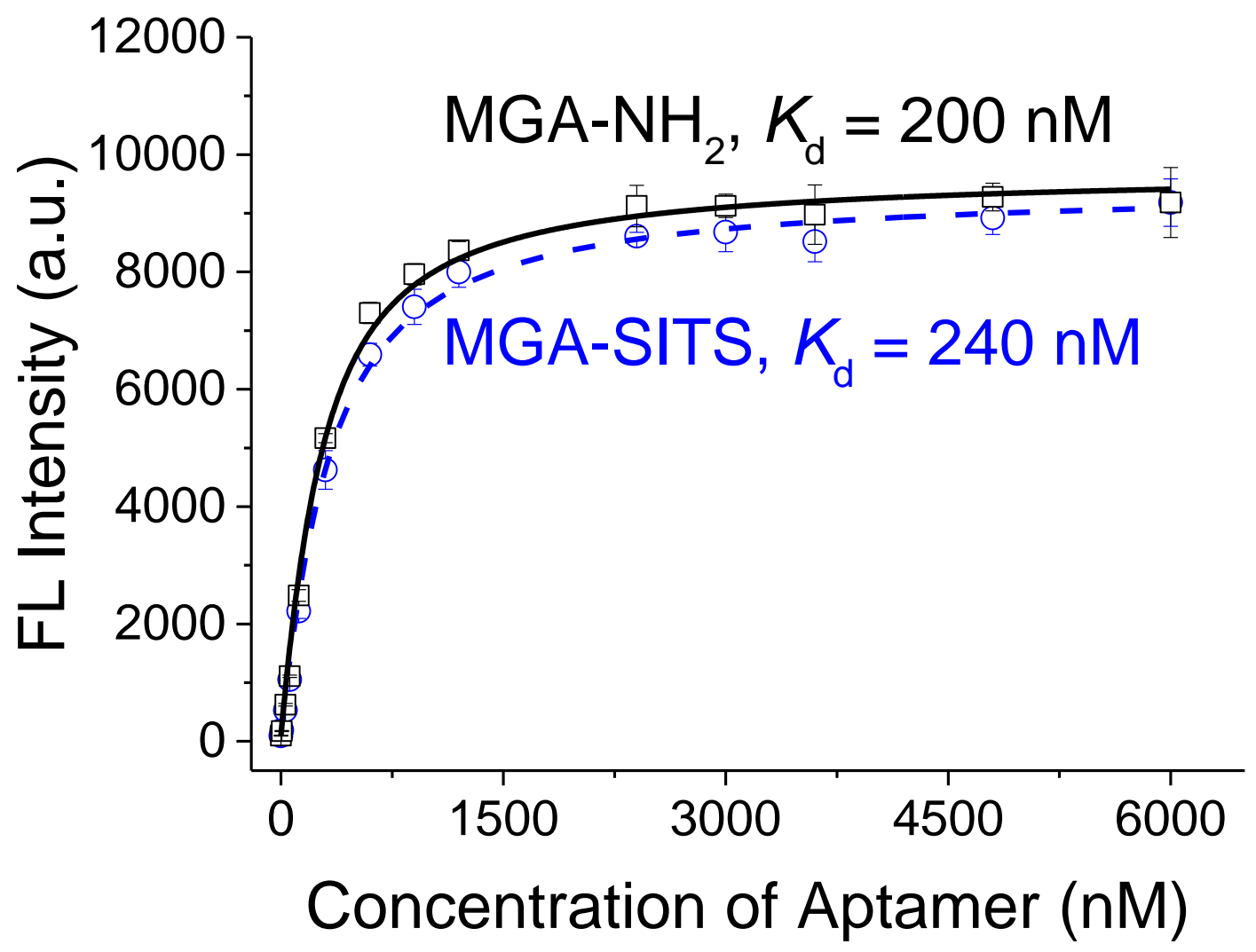

Figure 6.10 Binding of MGA- $\mathrm{NH}_{2}$ and MGA-SITS (5) with $\mathrm{MG}$, ex/em $=620 \mathrm{~nm} / 656 \mathrm{~nm}$.

\subsubsection{Fluorescence Decay of MGA-SITS Is Retarded upon MG Binding}

The MGA-SITS conjugate was incubated with the ligand MG, which should retard the stilbene's trans-cis photoisomerization according to our proposed hypothesis under the "aptamer-stilbene" design. Fluorescence decay of MG-SITS was measured according to the method in section 6.2.6 in the presence and absence of MG. As seen in Figure 6.11A,

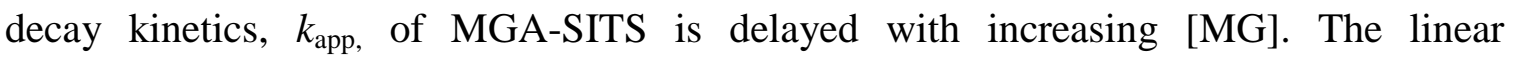
correlation of $k_{\text {app }}$ vs. [MG] suggests adaptive folding by MGA confines the free photoisomerization of the C38 SITS conjugate, resulting in an inhibited photodecay. NMR characterization by others has shown that MGA forms G1-C38 base pair under adaptive binding with MG [17]. Additionally, isomerization of trans-stilbene is subjected to the surrounding environment $[9,10]$. Thus, change in local environment induced by aptamer binding, or more specifically, increase in local viscosity is suspected to be the reason for the inhibition of SITS fluorescence decay, in the presence of MG. Considering 
the structures of SIST and the folded MGA, this impact on fluorescence decay may be attributed to hydrogen bond or stacking interactions between SITS and MGA, or both. Figure 6.11B presents the linear relationship between $k_{\text {app }}$ and MG concentration. From 0.5 to $50 \mu \mathrm{M}$, a good linear range can be obtained $\left(\mathrm{R}^{2}=0.98\right)$, with slope $=-9.4 \times 10^{-4} \mathrm{~s}^{-}$ ${ }^{1} \cdot \mu \mathrm{M}^{-1}$, and the limit of detection (LOD) for the sensing of MG is calculated to be 10.5 $\mu \mathrm{M}$ (defined as $3 \sigma$ criterion, $\sigma$ is the standard deviation of response). The results on $\mathrm{MG}$ sensing by the MGA-SITS conjugate illustrate a novel aptameric structure-switching strategy quantitating by fluorescence decay kinetics. However, as shown in Figure 6.11B, it should also be noted that the impact on MGA-SITS fluorescence decay is not dramatic as the concentration of MG increases ( response resulting in relatively low sensitive and high LOD $(10.5 \mu \mathrm{M})$ may be due to the relatively week fluorescence intensity. To improve the sensitivity, higher concentration of MGA-SITS may be used in the future, or through another more efficient way, functionalized stilbene with high fluorescence quantum yield should be employed for the conjugation. In summary, the results on MG sensing by the MGA-SITS provide preliminary proof-of-concept support towards our hypothesis.
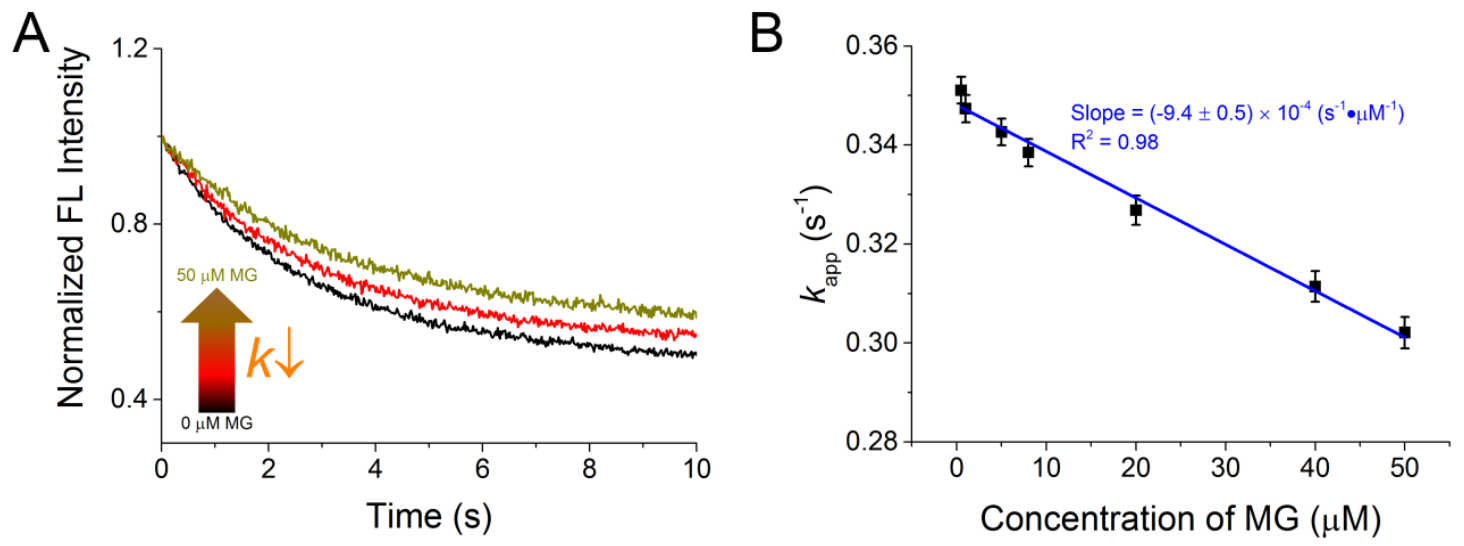

Figure 6.11 (A) Fluorescence decay curves of MGA-SITS (5) in the presence of increasing MG concentration. (B) Calibration plot of $k_{\text {app }}$ of MGA-SITS as a function of MG concentration $(0.5-50 \mu \mathrm{M})$.

\subsubsection{Delayed Fluorescence Decay Isolated To MGA-SITS}

MG inhibits the fluorescence decay of MGA-SITS as shown in the results of section 
6.3.4. In order to prove further support for our central hypothesis, we challenged the central hypothesis with two null-hypotheses pertaining to the delayed photoisomerization inhibition: 1) MG interaction with SITS is responsible for the fluorescence decay regardless of aptamer binding and 2) Wild type MGA/MG binding complex can inhibit the fluorescence decay of SITS through non-specific binding interactions. The nullhypotheses were conducted by monitoring the $k_{\text {app }}$ of SITS in the presence of MG or MGA/MG complex. Figure 6.12A presents the fluorescence decay curves of SITS with and without the addition of $\mathrm{MG}$ or $\mathrm{MGA} / \mathrm{MG}$ binding complex. Figure 6.12B compares the corresponding $k_{\text {app }}$ values of SITS in Figure 6.12A and shows no significant difference in the absence and presence of MG or MGA/MG complex (significance level $p$ $<0.05, \mathrm{n}=3$ ). Additionally, the $k_{\text {app }}$ of SITS is also independent of MG concentration, since the addition of $20 \mu \mathrm{M}$ or $40 \mu \mathrm{M}$ MG results in the similar $k_{\text {app }}$, regardless of the presence of MGA (Figure 6.12B). By contrast, MG leads to change in $K_{\text {app }}$ of MGASITS under the same experimental conditions (Figure 6.11A and Figure 6.11B). The results suggest the fluorescence decay inhibition on MGA-SITS induced by MG is not due to the interaction of SITS molecule with MG or MGA/MG complex when SITS is not grafted on aptamer. These evidences indicate the influence of MG on SITS fluorescence decay (Figure 6.11) is only owing to ligand induced aptameric folding of SITS attached MGA (illustrated in Figure 6.1), and the two null-hypotheses are reported false under the given conditions.
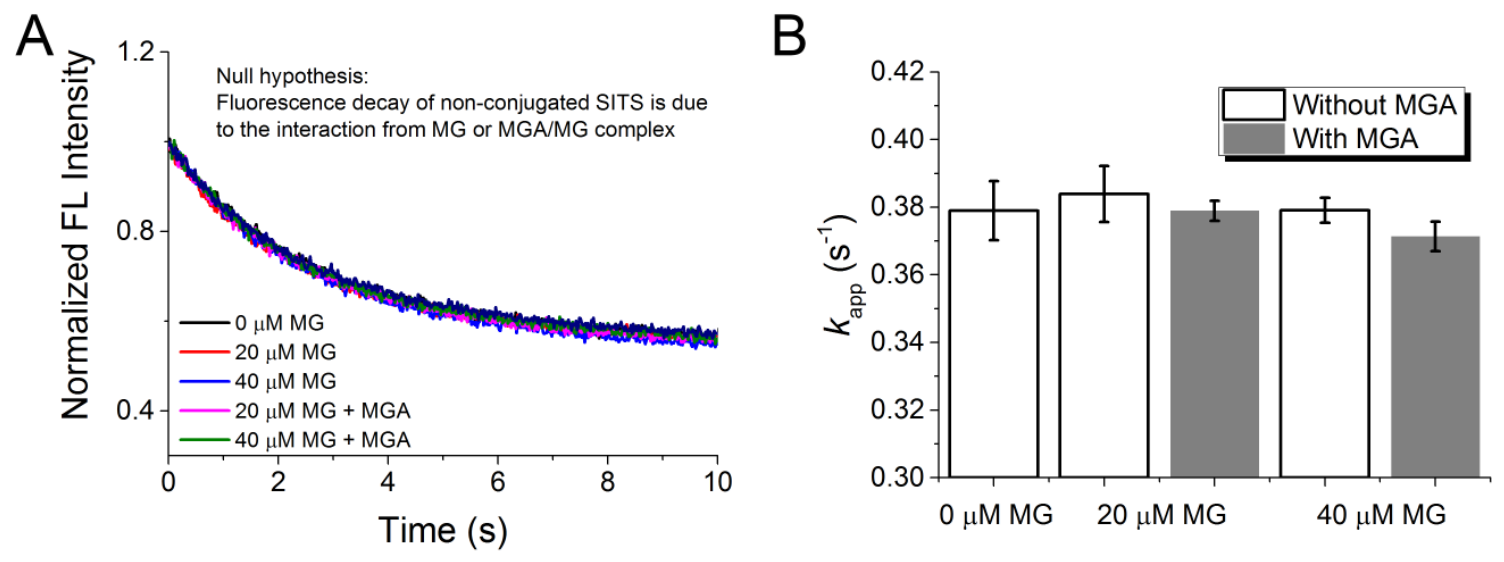

Figure 6.12 (A) Fluorescence decay curves of SITS (4) in the presence of MG and MGA/MG complex. (B) $k_{\text {app }}$ of SITS upon the presence of MG and MGA/MG complex. *: $p<0.05$ : 
significant difference against SITS control (without MG and MGA) $(n=3)$.

\subsubsection{The MGA-SITS Conjugate Shows Selectivity of Ligands}

Other triphenyl dyes RhB and TMR (see Figure 6.2 for structures) similar to MG were tested regarding to the selectivity and multiplex detection of the MGA-SITS sensor. RhB does not specifically bind with MGA (section 5.3.2) while TMR binds with MGA with high affinity [18]. There is no significant change in MGA-SITS fluorescence decay in the presence of $20 \mu \mathrm{M} \mathrm{RhB}$, while the decay is dramatically inhibited in the presence of 20 $\mu \mathrm{M}$ TMR, which displays a more sensitive than all known concentrations of MG (Figure 6.13A). As shown in Figure 6.13B, analysis on $k_{\text {app }}$ of MGA-SITS quantitatively confirms the observations in Figure 6.13A. Against MGA-SITS blank control, there are significant differences on $k_{\text {app }}$ in the presence of MG and TMR, but no significant difference $k_{\mathrm{app}}$ in the presence of $\mathrm{RhB}$ (significance level $p<0.05$ ), which displays a good selectivity of the MGA-SITS sensor.
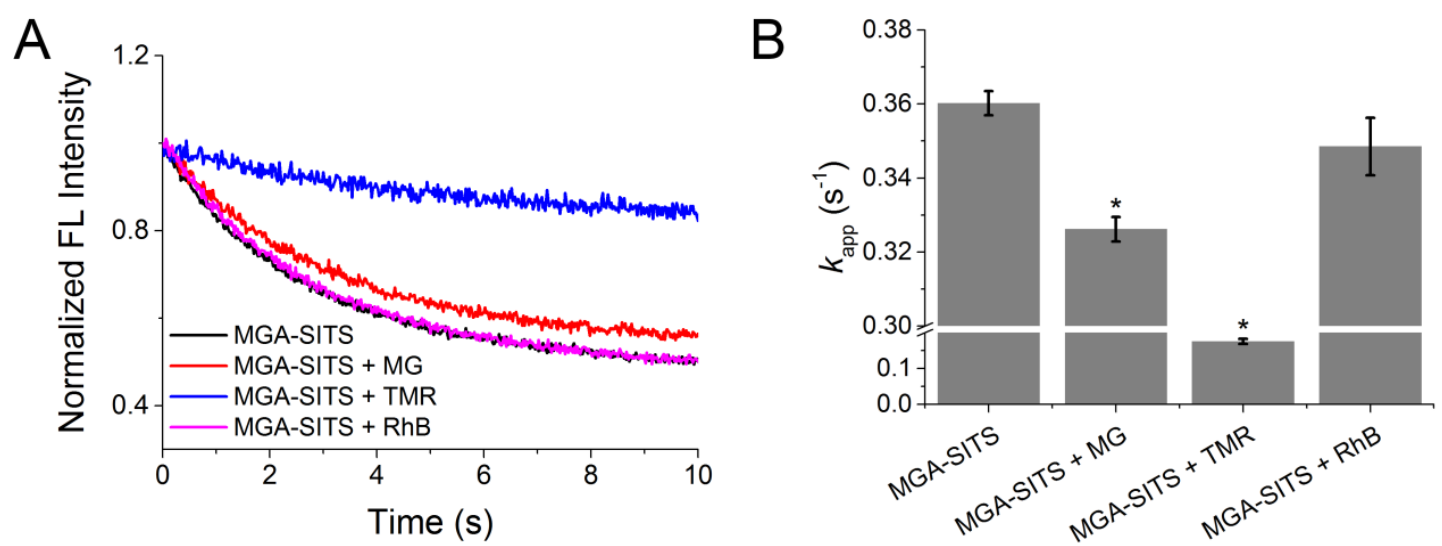

Figure 6.13 (A) Fluorescence decay curves of MGA-SITS (5) in the presence of $20 \mu \mathrm{M}$ MG, TMR (15) and RhB (10). (B) $k_{\text {app }}$ of MGA-SITS upon the addition of the analytes. *: $p<0.05$ : significant difference against MGA-SITS $(n=3)$.

TMR displays a greater retardation in fluorescence decay of the MGA-SITS conjugate. Similar to the case of MG sensing, the decay kinetics of MGA-SITS decreases upon its binding to TMR (Figure 6.14A). Linear correlation of $k_{\text {app }}$ Vs. TMR concentration gives a slope of $-1.4 \times 10^{-2} \mathrm{~s}^{-1} \cdot \mu \mathrm{M}^{-1}\left(\mathrm{R}^{2}=0.92\right)$ ranging from 0.2 to $20 \mu \mathrm{M}$, which is 15 times 
more sensitive than the MG linear fit $\left(-1.4 \times 10^{-2}\right.$ vs. $\left.-9.4 \times 10^{-4}\right)$. LOD of TMR is calculated to be $0.7 \mu \mathrm{M}$ (Figure 6.14B). In short, TMR sensing by the MGA-SITS sensor presents more than 10-fold higher sensitivity compared to MG sensing, with LOD in submicromolar level. From the reported data, TMR has up to 5 times smaller $K_{\mathrm{d}}$ value than MG in the binding with MGA $[15,18]$, which may be a potential reason for the higher sensitivity of TMR linear correlation. TMR's strong binding affinity with MGA-SITS may induce more folded aptamer. In addition, the structural feature of MGA/ligand complex may be another reason. MGA presents different bound structure when it binds to TMR and MG [17,18], which may induce a different impact on stilbene isomerization. This speculation needs to be examined by computational modelling in the future work. This difference between the sensing of MG and TMR gives indication on the future design of the sensor based on the hypothesis, especially for the improvement of the sensitivity. For instance, employ MGA-SITS/TMR complex for the sensing of MG by competitive binding, which might induce a higher sensitivity in MG detection. Additionally, optimizations of binding conditions for higher affinity may also be beneficial for the sensitivity enhancement.
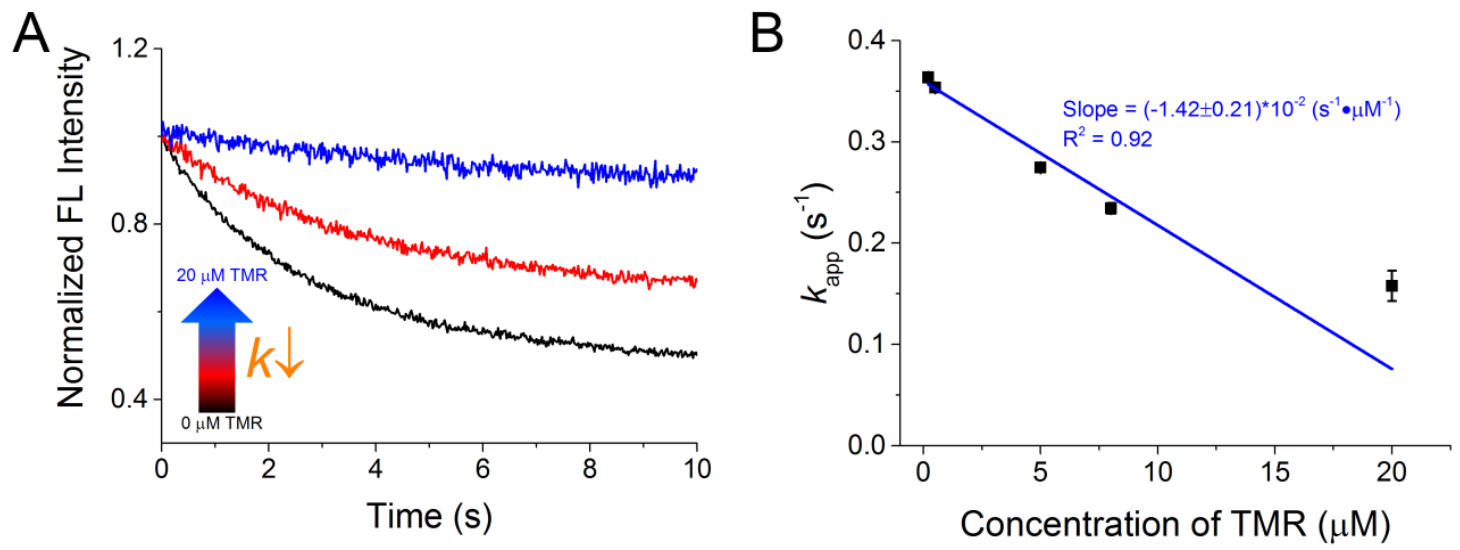

Figure 6.14 (A) Fluorescence decay curves of MGA-SITS (5) in the presence of increasing concentration of TMR (15). (B) Calibration plot of MGA-SITS $k_{\text {app }}$ as a function of TMR concentration $(0.2-20 \mu \mathrm{M})$.

\subsection{Conclusions}


In this work, we have developed a MGA-SITS conjugate which can challenged our central hypothesis under the scope design "stilbene-aptamer conjugate fluorescence decay correlates to analyte binding". This design takes advantages of conformational changes present upon aptamer binding and stilbene's fluorescence decay sensitivity. The MGA-SITS aptasensor developed is capable of selectivity toward a range of ligand affinities. Difference in response between ligands of TMR and MG sensing suggests high binding affinity and competitive design of MG to MGA-SITS/TMR complex may result in a higher sensitivity, giving indications for future strategies on the sensor optimization. Additionally, influence on SITS fluorescence decay from the interactions with MG or MGA/MG complex is ruled out by two null hypotheses. A novel fluorescence decay based sensing mode has been reported for the first time. Future work should focus on the improvement in sensitivity of the aptasensor like MG competitive binding with MGASITS/TMR complex, grafting stilbene on various positions of MGA, analysis of stilbene photoisomerization on the bound aptamer, employing functionalized stilbene with high fluorescence quantum yield, improving the bioconjugation efficiency, and enhancing the binding affinity.

\section{References:}

[1] J.-W. Chen; X.-P. Liu; K.-J. Feng; Y. Liang; J.-H. Jiang; G.-L. Shen; R.-Q. Yu Biosensors and Bioelectronics 2008, 24, 66.

[2] D. Li; S. Song; C. Fan Accounts of Chemical Research 2010, 43, 631.

[3] J. Zhang; X. Zhang; G. Yang; J. Chen; S. Wang Biosensors and Bioelectronics 2013, $41,704$.

[4] M. A. D. Neves; C. Blaszykowski; M. Thompson Analytical Chemistry 2016, 88, 3098.

[5] F. B. Mallory; C. S. Wood; J. T. Gordon; L. C. Lindquist; M. L. Savitz Journal of the American Chemical Society 1962, 84, 4361.

[6] D. H. Waldeck Chemical Reviews 1991, 91, 415.

[7] O. Chen; R. Glaser; G. I. Likhtenshtein Journal of Biochemical and Biophysical Methods 2008, 70, 1073. 
[8] N. Strashnikova; V. Papper; P. Parkhomyuk; G. I. Likhtenshtein; V. Ratner; R. Marks Journal of Photochemistry and Photobiology A: Chemistry 1999, 122, 133.

[9] A. Parthasarathy; L. S. Kaanumalle; V. Ramamurthy Organic Letters 2007, 9, 5059.

[10] M. R. Ams; D. Ajami; S. L. Craig; J.-S. Yang; J. Rebek, Jr. Beilstein Journal of Organic Chemistry 2009, 5, 79.

[11] G. T. Hermanson In Bioconjugate Techniques (Third edition); Academic Press: Boston, 2013, p 229.

[12] O. Pokholenko; A. Gissot; B. Vialet; K. Bathany; A. Thiery; P. Barthelemy Journal of Materials Chemistry B 2013, 1, 5329.

[13] C. M. Castleberry; C.-W. Chou; P. A. Limbach In Current Protocols in Nucleic Acid Chemistry; John Wiley \& Sons, Inc.: 2008.

[14] J. R. Babendure; S. R. Adams; R. Y. Tsien Journal of the American Chemical Society 2003, 125, 14716.

[15] S. L. Stead; H. Ashwin; B. H. Johnston; A. Dallas; S. A. Kazakov; J. A. Tarbin; M. Sharman; J. Kay; B. J. Keely Analytical Chemistry 2010, 82, 2652.

[16] R. E. Wang; Y. Zhang; J. Cai; W. Cai; T. Gao Current Medicinal Chemistry 2011, 18,4175 .

[17] J. Flinders; S. C. DeFina; D. M. Brackett; C. Baugh; C. Wilson; T. Dieckmann ChemBioChem 2004, 5, 62.

[18] C. Baugh; D. Grate; C. Wilson Journal of Molecular Biology 2000, 301, 117. 



\section{Chapter 7}

\section{Summary, Implications and Future Suggestions}

This chapter summarizes the results throughout the thesis, and presents implications from the outcomes. Both the successful and failed data in this thesis give significant hints on future designs of the fluorescence decay based biosensors, incorporating the adaptive binding of aptamer and fluorescence decay of stilbene. Based on the results obtained and literature, some strategies are proposed for future work, such as the employment of computational molecular modelling on optimal malachite green (MG)stilbene synthesis, involvement of new click chemistry for higher reaction efficiency, regeneration of the sensor and competitive binding of $M G$ to MGA-SITS/TMR complex. These strategies should be able to improve the sensing performance of the fluorescence decay based aptasensor. 


\subsection{Summary}

Combing the adaptive binding behavior of aptamer and the fluorescence decay property of stilbene, an aptasensor quantitating by fluorescence decay has been proposed in this work. Fluorescence decay quantitation may overcome some disadvantages in typical "signal on/off" fluorescent biosensors, such as the laborious and tedious washing procedures for the separation of adjacent fluorophores and being susceptible to interference like autofluorescence. Owing to the reported merits, malachite green aptamer (MGA) and its ligand malachite green (MG) were chosen as a model study to test the hypothesis of this thesis. MG was reported to have significant fluorescence enhancement up on MGA binding [1], which can be utilized for the determination of binding affinity. However, the MGA/MG binding was found to be unstable over time, as evidenced by the variation on apparent equilibrium dissociation constant $\left(K_{\mathrm{d}}\right)$ and observed fluorescence intensity, due to the hydrolysis of MG. This phenomenon may introduce difficulties in our hypothesis testing, especially in accurate detection of MG during long-time operations. Organic additives acetonitrile (ACN), dimethyl sulfoxide (DMSO) and ethanol $(\mathrm{EtOH})$ were found to prevent $\mathrm{MG}$ hydrolysis, and thus stabilize the MGA/MG binding, with an aptamer associated synergistic stabilizing mechanism proposed [2]. This discovery may also be beneficial for carbocation aptamer ligands susceptible to $S_{N} 1$ hydrolysis. Additionally, other factors such as $\mathrm{pH}$ and the stability of MGA were also investigated to obtain optimal conditions for $\mathrm{MGA} / \mathrm{MG}$ binding. The total outcomes

obtained pave the foundation and provide optimal experimental conditions for the hypothesis testing.

To test our hypothesis, two designs were investigated within the scopes: 1) "competitive stilbene-analyte conjugate with analyte" and 2) "stilbene-aptamer conjugate fluorescence decay correlates to analyte binding". The design 1) "competitive stilbene-analyte conjugate with analyte" focuses on the performance of MG-stilbene conjugate. It is supposed that MG-stilbene conjugate is able to keep both the binding of MG (with MGA) and fluorescence decay of stilbene, which are the critical properties for the investigation of the hypothesis. Thus, MG-stilbene conjugates have been studied towards their MGA 
binding and stilbene fluorescence decay, including a rhodamine $\mathrm{B}(\mathrm{RhB})$-stilbene conjugate as a negative control. Studies on negative control RhB-stilbene conjugate give some indications: 1) fluorescence decay of stilbene in MG-stilbene conjugates is retained, 2) No evidence of MGA non-specific binding is observed with stilbene conjugates and 3) no significant difference is seen between the fluorescence decay of stilbene conjugate and its non-binding aptamer mixture. This RhB-stilbene conjugate showing no binding (with MGA) and significant fluorescence decay provides a good negative control on the MGstilbene conjugate design. The structure activity relationships of conjugate binding to aptamer and photoisomerization led to conjugates that allow ongoing proof of concept testing. In particular, structure activity relationships were focused on conjugate covalent linkers and stilbene side groups. MG-COO-stilbene- $\mathrm{NO}_{2}$ conjugate displays extremely low binding affinity with MGA and no detectable fluorescence decay (as expected). Conjugates $\mathrm{MG}-\mathrm{CH}_{2}-\mathrm{NH}$-stilbene-OMe and $\mathrm{MG}_{-} \mathrm{CH}_{2}-\mathrm{NAc}$-stilbene-OMe exhibit fluorescence decay, but the binding affinity is still relatively low compared to MG, which is not ideal for the hypothesis testing. MG-fused stilbene conjugate with smaller molecular size solves the problem in binding affinity, with $K_{\mathrm{d}}$ comparable to $\mathrm{MG}$, but it loss the fluorescence decay property. Although no ideal MG-stilbene conjugate candidate is obtained for hypothesis testing, some indications on structure activity relationships can be referred from the performance of the conjugates, associated with their molecular properties (Table 5.1). By analysis of the conjugates structure, smaller molecular size, introduction of decoupling groups between $\mathrm{MG}$ and stilbene, and strengthening of electrostatic interaction with MGA may be beneficial for enhancing the binding affinity of MG-stilbene conjugates. It has been reported that electrostatic and base pair stacking interaction are the two main forces in MGA/MG interactions [3,4] On the other hand, introduction of electron donating groups as side groups may help keep the fluorescence decay property. The results show the MG-stilbene conjugate molecules possess either low binding affinity or weak fluorescence decay, which limits its design as a sensitive aptasensor, but provides proof of concept data of the design.

According to design 2) "stilbene-aptamer conjugate fluorescence decay correlates to analyte binding", stilbene molecule needs to be grafted on aptamer to fabricate the 
biosensor. Aptamer-stilbene conjugate MGA-SITS was synthesized by classical amineisothiocyanate click chemistry in aqueous environment, under the reaction conditions optimized. Matrix-assisted laser desorption/ionization time-of-flight (MALDI-TOF) and high performance liquid chromatography (HPLC)-diode array detector (DAD) have confirmed the successful conjugation and complete removal of the free starting stilbene molecule. The MGA-SITS conjugate has comparable binding affinity (compared to MGA- $\mathrm{NH}_{2}$ ) and exhibits significant fluorescence decay similar to the starting SITS, which is available for the hypothesis testing. Thus, MG was subsequently tested by the MGA-SITS conjugate. Significantly, the presence of MG results in inhibition on the fluorescence decay of the MG-SITS conjugate, with quantitative reduced apparent fluorescence decay rate constant $\left(k_{\mathrm{app}}\right)$. This novel discovery supports the hypothesis of the thesis under our limited experimental conditions within the scope (Figure 6.1). Results from negative control experiments on SITS have ruled out the interference form interactions between MG and SITS, or between MGA/MG complex and SITS, which support that the inhibition on fluorescence decay is only due to SITS grafted MGA binding. Moreover, the fluorescence decay of MGA-SITS is only sensitive to MGA ligands $\mathrm{MG}$ and tetramethylrosamine (TMR), but not to the non-binding triphenyl compound $\mathrm{RhB}$, showing selectivity and the capability of multiplex detection of ligands. Linear ranges of $0.5-50 \mu \mathrm{M}$ for $\mathrm{MG}$ and $0.2-20 \mu \mathrm{M}$ for TMR have been obtained by the MGA-SITS conjugate sensor. Compared to the sensing of MG, sensing of TMR by MGA-SITS presents higher sensitivity and lower limit of detection (LOD). This difference in response indicates that higher affinity or different bound configuration of MGA may result in a better sensing performance, which also gives suggestions on the further optimization of aptasensors based on this "aptamer-stilbene" design, for example, the competitive binding of MG to MGA-SITS/TMR complex. A novel sensing mode based on fluorescence decay kinetics has been invented according to the hypothesis of the thesis. This mode takes advantages of conformational change of aptamer binding and fluorescence decay of stilbene by grafting stilbene molecule on aptamer. However, sensitivity and LOD of the aptasensor still needs to be improved by further optimization. The results supporting the hypothesis set up a significant starting point for the development of fluorescence decay based aptasensor. 


\subsection{Implications and Future Suggestions}

Besides the discovery and outcomes achieved in the study, some indications are obtained from the experimental results, which should be valuable for the future design of the aptasensors based on the hypothesis of this thesis. Additionally, some strategies and designs will also be suggested, which may be beneficial for the construction of fluorescence decay based aptasensors according to our hypothesis.

For the design 1) "competitive stilbene-analyte conjugate with analyte", some implications can be obtained from the results, which may help improve the performance of MG-stilbene conjugates. Electron accepting groups (i.e. -COO- and $\mathrm{NO}_{2}$ groups) should be avoided for stilbene side groups, which may decrease the fluorescence decay. In other words, electron donating groups (like $-\mathrm{OCH}_{3}$ ) are preferred for stilbene fluorescence decay retaining. Additionally, small molecular size of conjugate is preferentially recommended but the fused $\mathrm{MG}$ and stilbene may heavily disturb the fluorescence properties of stilbene. Finally, increase of electrostatic effect and spacers preventing the MG from electron disturbing groups should be considered to enhance the aptamer binding. The evidences on structure activity relationships above will be beneficial for the synthesis of new MG-stilbene conjugates in the future.

Some other strategies are also suggested for the future work. The first idea is to apply computational molecular modelling to guide the synthesis. To date, NMR and XRD structure of $\mathrm{MG}$ or TMR bound MGA have been revealed [3,4]. In fact, some computational molecular modelling studies on MGA/MG complex have been reported, which presents the configuration of MGA and the conformation and circumstances of the bound MG [5-8]. Compared with practical lab synthesis, computational modelling dramatically saves time, labor force and materials. Additionally, some preliminary results on MG-stilbene have been obtained in this study as described above, which may be beneficial for improving the modelling parameters. Thus, employment of the data we have to computational modelling may give helpful hints and implications on the future 
synthesis of MG-stilbene conjugates in keeping both the MG binding and stilbene fluorescence decay properties. In turn, the experimental results on new synthesis MGstilbene conjugate can also put some feedbacks on the computational modelling (Figure 7.1). Finally, incorporation of cycles of synthesis and modelling will create a database, which presents guideline on the synthesis of MG-stilbene conjugates suitable for hypothesis investigation as well as other aptamer ligand-stilbene conjugates.

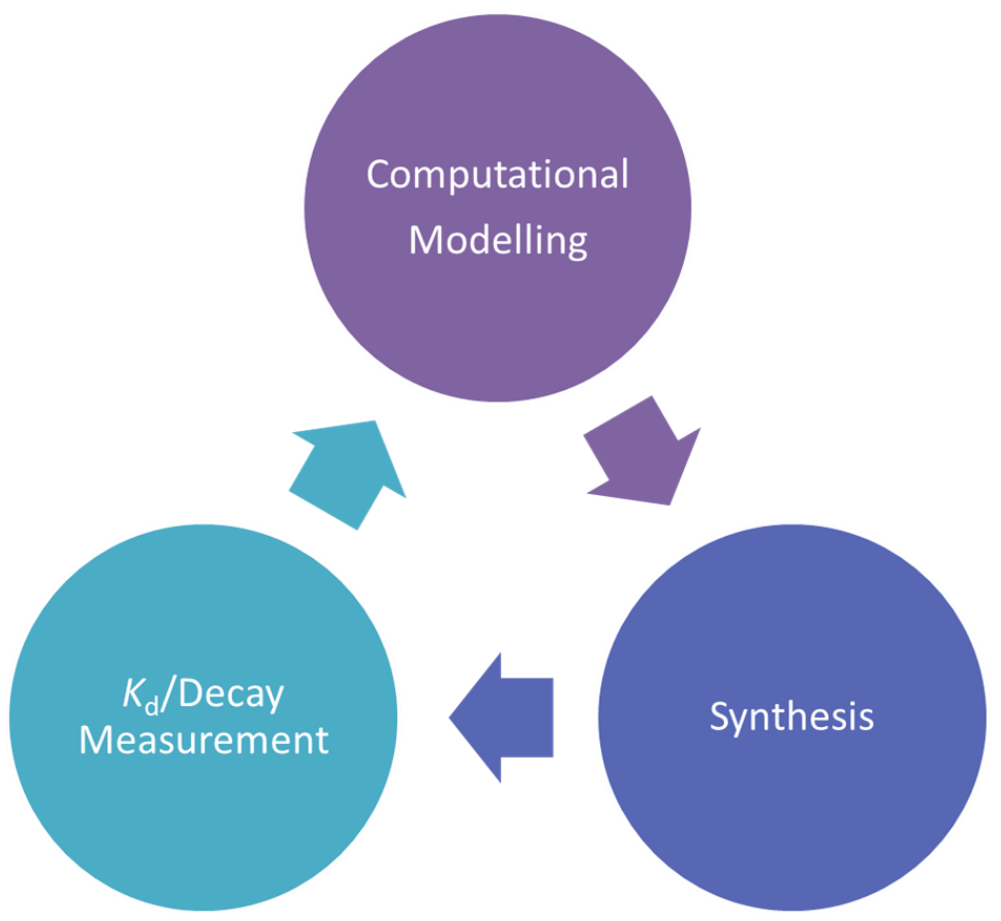

Figure 7.1 Recycling of computational modelling and synthesis on MG-stilbene conjugates for optimal binding affinity and fluorescence decay.

Another proposal is to conduct a new round of systematic evolution of ligands by exponential enrichment (SELEX) selection. The purpose is to obtain a new aptamer simultaneous for both MG and the MG-stilbene conjugate with fluorescence decay (i.e. MG- $\mathrm{CH}_{2}-\mathrm{NAc}$-stilbene-OMe), and there requires competitive binding between the two ligands in the aptamer. The example of MGA has shown the possibility that aptamer can bind to multiple ligands. This strategy removes the consideration of keeping the binding affinity of MG-stilbene conjugates, since the aptamer binding both ligands will be selected post the synthesis of MG-stilbene conjugate. On the other hand, selection of aptamer with competitive binding of MG and stilbene is another strategy. If an aptamer 
binds to both MG and stilbene, then MG can release the pre-bound stilbene molecular by competitive binding and probably change the fluorescence kinetics of stilbene due to the variation of micro-environment rearrangements. However, it might be challenging to obtain this kind of aptamer simultaneous binding to two targeted ligands in practical operations.

For the design 2) "stilbene-aptamer conjugate fluorescence decay correlates to analyte binding", hypothesis has been supported by data under the scope and our experimental conditions. However, there are still some issues need to be solved and some further development should be conducted in the future.

The sensitivity of the MGA-stilbene sensor is relatively low, and thus more effort should be made to enhance the sensitivity. According to the sensing results from MG and TMR by the MGA-SITS conjugate sensor, aptamer-ligand with higher binding affinity and or different bound configuration of MGA may be preferred for higher sensitivity. Optimizations for enhancing the binding affinity should be an approach to enhance the conjugate sensitivity. Additionally, some modification on stilbene molecule may be performed to enhance the fluorescence properties of the conjugate and subsequently enhance the sensitivity, as higher quantum yield and $k_{\text {app }}$ of the stilbene molecule can reduce the minimum amount of conjugate sensor needed for sensing, which may result in a stronger response to aptamer ligands. Additionally, decoration of stilbene molecules (i.e. introduction of electrostatic strengthening groups) may increase the electrostatic, base stacking or hydrogen bonding interactions between the stilbene molecule and the fold MGA, which results in a strong inhibition of stilbene fluorescence decay and thus enhances the sensitivity. However, the real situation of stilbene in the bound MGA has not been reported yet. NMR or XRD study on the MGA-SITS before and after aptamer binding should be conducted in the future to reveal the configuration of stilbene and the mechanism on how the aptamer folding affects the stilbene isomerization. Furthermore, computational molecular modelling on the dynamic change of stilbene during aptamer binding should also help discover the mechanism and give indications on optimal designs on the aptamer-stilbene conjugate biosensors. 
Competitive binding strategy may be helpful in enhancing the sensitivity of the conjugate. According to our results, MGA-SITS is more sensitive to TMR compared to MG. Thus, MG sensing strategy by releasing the TMR from prebound MGA-SITS/TMR complex is proposed for the future work and Figure 7.2 shows the concept. In this design, MG will replace the TMR in MGA-SITS/TMR complex. The decrease of MGA-SITS/TMR complex will dramatically promote the fluorescence decay, while the increase of MGASITS/MG complex will have relatively limited impact on the inhibition of fluorescence decay, due to the difference in MGA-SITS conjugate sensitivity for TMR and MG. This design may results in a higher sensitivity for MG sensing.

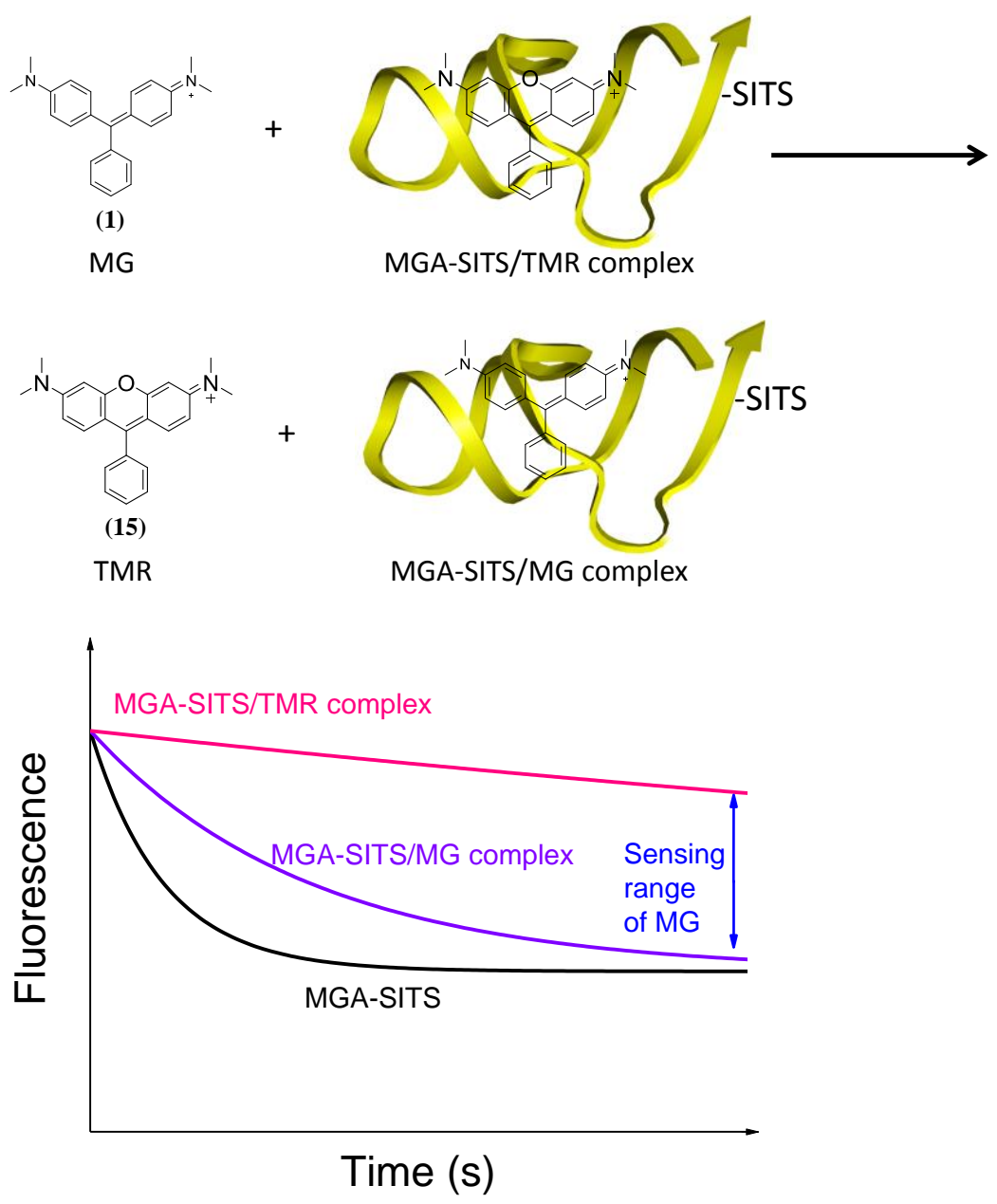

Figure 7.2 Competitive binding of MG with prebound MGA-SITS/TMR complex for MG sensing. 
The bioconjugation reaction is still inefficient, which increase the cost of the aptasensor. The remained not reacted $\mathrm{MGA}-\mathrm{NH}_{2}$ may bind to ligands and thus reduce the sensitivity. Future work should also be focused on increasing the reaction efficiency, for instance, using other bioconjugation strategies. Maleimide-thiol reaction is a click reaction with high efficiency. My project partner Ms. Wu Yuanyuan (PhD candidate) has conducted the conjugation of $\mathrm{MeO}$-stilbene-maleimide (20) with thiol molecule 6-Mercapto-1hexanol $(\mathrm{MCH})(\mathbf{2 1})$, which mimics the reaction of maleimide stilbene with SH-aptamer (Figure 7.3). The MeO-stilbene-maleimide-MCH (22) conjugate displays significant fluorescence decay in aqueous environment, which implies the possible conjugation of MeO-stilbene-maleimide (20) with thiol modified aptamer, with fluorescence decay behavior on the conjugate. On the other hand, C38 thiol modified MGA (MGA-SH) binds to $\mathrm{MG}$ with $K_{\mathrm{d}}=980 \mathrm{nM}$ from our preliminary study (Figure 7.4), which enables the possible application of the MGA-SH with guaranteed binding affinity after the aptamer is conjugated with $\mathrm{MeO}$-stilbene-maleimide (20). According to the results above, conjugation of MGA-SH and maleimide stilbene molecules (like MeO-stilbenemaleimide (20)) can be conducted in the future, in order to reach higher reaction efficiency as well as better sensing performance.
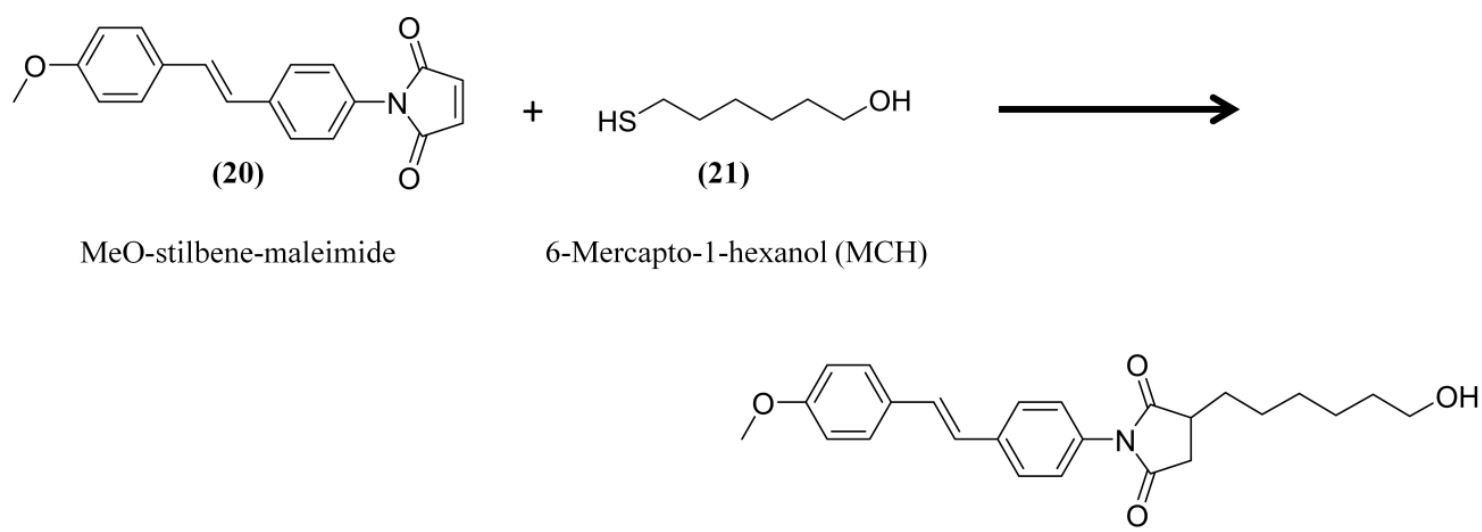

(22)

MeO-stilbene-maleimide-MCH

Figure 7.3 Conjugation of MeO-stilbene-maleimide (20) with 6-Mercapoto-1-hexanol (MCH) (21). 


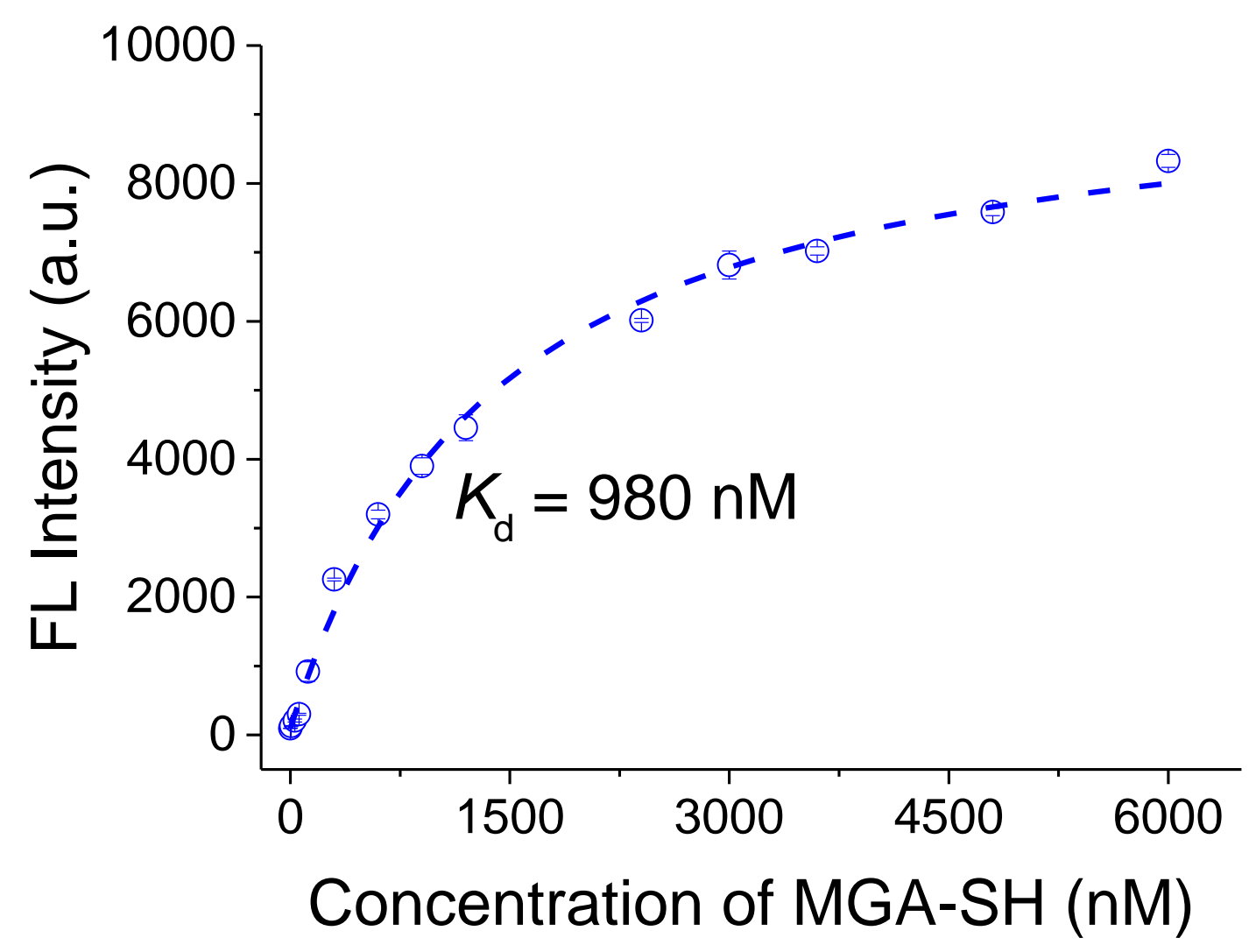

Figure 7.4 MGA-SH/MG binding curve and its calculated $K_{\mathrm{d}}$, ex/em $=620 \mathrm{~nm} / 656 \mathrm{~nm}$.

In addition, different positions on aptamer for stilbene grafting should also be considered, which may vary the sensing performance, for instance, increasing the binding affinity or the response to ligands of the MGA-stilbene biosensor. It has been reported by Flinders et al that some base mutants on MGA only results in tiny impact on its binding affinity [4]. Thiol modified MGA on various positions (U4, A9, C20 and C38) have also been investigated by us, showing applicable binding affinities (less than $1000 \mathrm{nM}$ ) with MG (Table 6.1). The conjugation of stilbene on different positions of MGA may results in different efficiency of stilbene photoisomerization, which may enhance the sensitivity of the conjugate. Thus, by grafting stilbene on suitable location of the MGA, it can be proposed that the binding will not be influenced significantly. However, the fluorescence decay behavior of stilbene molecule may be different due to the different surround chemical and biological environment. Especially, the different fluorescence decay of stilbene molecule in different positions may reveal a law or relationship, which enables flexible tuning on fluorescence decay based on specific requirements. Furthermore, 
owing to the well-known NMR and XRD 3D structure of the aptamer, computational molecular modelling should also help on implying the properties of MGA-stilbene when the stilbene molecule is grafted on different base of the MGA.

Computational molecular modelling may also help reveal the different response of the sensor in the sensing of MG and TMR. Sensing of TMR displays higher sensitivity and lower LOD compared to the MG sensing. It is hypothesized in Chapter 6 that may be due to the higher binding affinity and or different bound configuration of MGA in TMR binding. Computational modelling can be used to examine the hypothesis. By computational modelling, the view of the stilbene surrounding environment can be obtained, revealing the interaction between aptamer and stilbene (i.e. steric stacking and hydrogen bonding). Additionally, the freedom of stilbene isomerization can also be monitored upon the MGA-SITS binding to ligands, and the different stilbene surrounding environment induced by MG and TMR should be a key point to explain why the two ligands result in different fluorescence decay responses.

Moreover, although the MGA-stilbene biosensor quantitating by fluorescence decay has been developed, the use of RNA aptamer may cause some problem in the practical applications. RNA is susceptible to ribonuclease degradation, while DNA is known to have much better stability. Thus, to achieve a robust biosensor, DNA aptamer should be preferential under the same conditions. Additionally, the price for DNA is normally much lower than that of RNA, and the yield of DNA is higher in synthesis. Recently, Wang et al has successfully selected DNA aptamer for MG [9]. It is worth applying the same strategies in this work to the DNA MG aptamer for the development of a more practical and robust aptasensor, which also reduces the cost in sensor fabrication.

Furthermore, a reusable device is prior to a single-used one. Thus, regeneration of the used MGA-SITS sensor is also important. In fact, it is possible to reuse the MGA-SITS sensor because both the MGA and trans-stilbene can be regenerated. Aptamers can undergo reversible thermal denaturation (different from antibodies). Normally, denaturation by heat can destroy the bound aptamer/ligand complexed structure and thus 
regenerate the aptamer for multiple uses [10]. Aptamer can even be regenerated by washing procedures when it binds to small molecule like cocaine $[11,12]$. On the other hand, the reversible trans-cis switching of stilbene compounds makes it an ideal reporter element in sensing. Even though the conversion of trans-stilbene to cis-stilbene is a relatively complete process with a high reaction rate, the cis form can be turned back to trans form under certain condition, e.g. heating or UV-Vis irradiation in another wavelength. Study shows that this "turning back" conformational change makes stilbene molecule recover fluorescent to almost the initial level [13]. Figure 7.5A presents the regeneration of aptamer and Figure 7.5B displays the cis to trans conversion of stilbene, which may towards the regeneration of aptamer-stilbene conjugate sensor (Figure 7.5C). Since both aptamer and stilbene can be regenerated for reusing, future work is suggested to regenerate the used aptamer-stilbene biosensor for multiple uses, which can reduce the cost of quantitation. If possible, approaches and conditions able to simultaneously regenerate the aptamer and stilbene are preferred, for instance, heating the used sensor with a proper temperature.

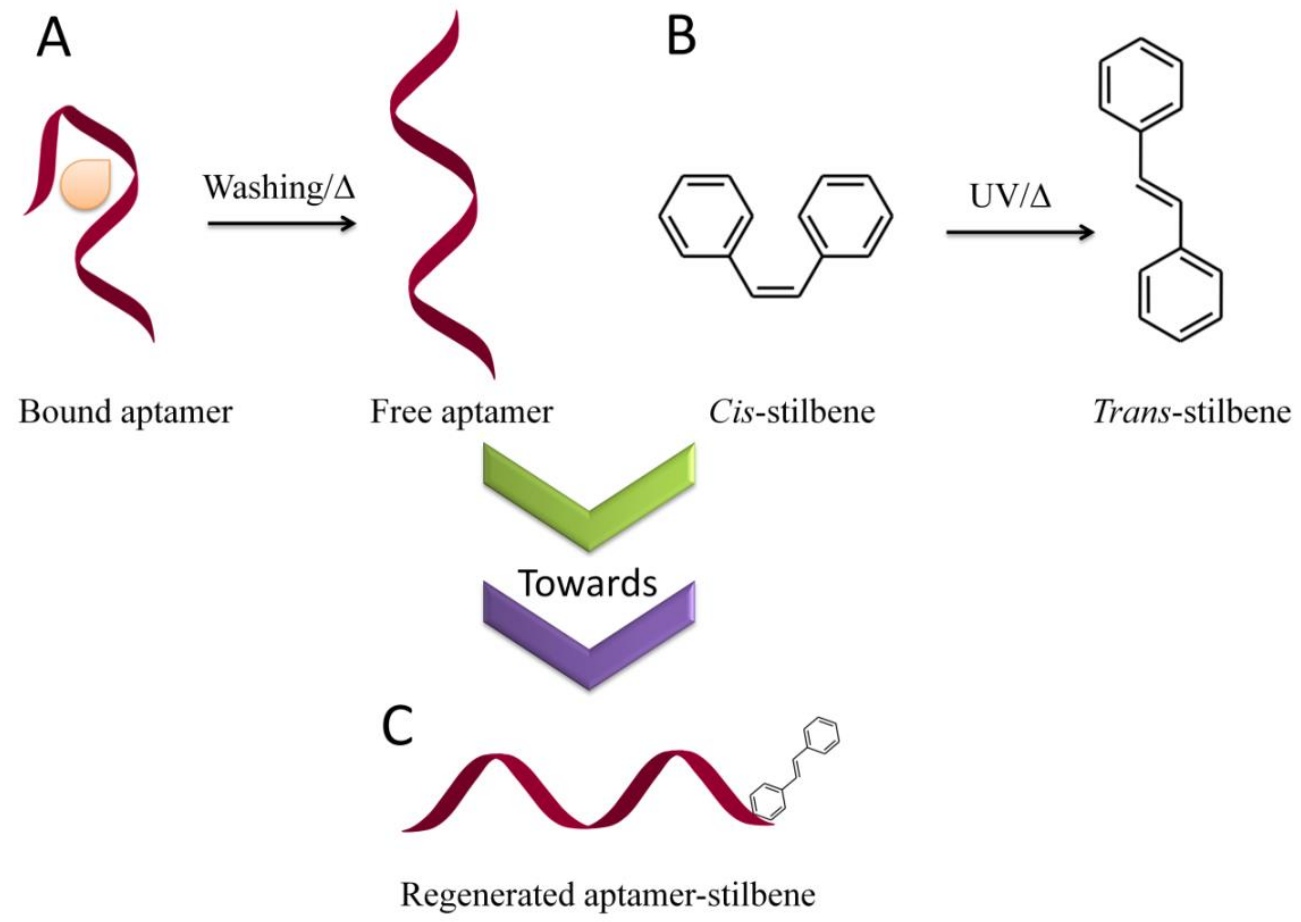

Figure 7.5 Schematic illustration of regeneration of aptamer (A), stilbene (B) and the regenerated aptamer-stilbene conjugate sensor (C). 
Finally, if possible, apply this strategy to other aptamers to see if this sensing mode can be used in other aptamers. Especially, aptamers with high binding affinities and with small organic molecule ligands should be tried based on the results obtained in this work. Successful employment of this fluorescence decay based strategy will further support the hypothesis of the thesis.

\section{References:}

[1] J. R. Babendure; S. R. Adams; R. Y. Tsien Journal of the American Chemical Society 2003, 125,14716 .

[2] Y. Zhou; H. Chi; Y. Wu; R. S. Marks; T. W. J. Steele Talanta 2016, 160, 172.

[3] C. Baugh; D. Grate; C. Wilson J Mol Biol 2000, 301, 117.

[4] J. Flinders; S. C. DeFina; D. M. Brackett; C. Baugh; C. Wilson; T. Dieckmann Chembiochem 2004, 5, 62.

[5] D. H. Nguyen; S. C. DeFina; W. H. Fink; T. Dieckmann Journal of the American Chemical Society 2002, 124, 15081.

[6] D. H. Nguyen; T. Dieckmann; M. E. Colvin; W. H. Fink The Journal of Physical Chemistry B 2004, 108, 1279.

[7] T. Wang; J. A. Hoy; M. H. Lamm; M. Nilsen-Hamilton Journal of the American Chemical Society 2009, 131, 14747.

[8] M. Hirabayashi; H. Ohashi; T. Kubo Journal of Computational and Theoretical Nanoscience 2010, 7, 831.

[9] H. Wang; J. Wang; N. Sun; H. Cheng; H. Chen; R. Pei ChemistrySelect 2016, 1, 1571.

[10] J.-G. Walter; F. Stahl; T. Scheper Engineering in Life Sciences 2012, 12, 496.

[11] J.-O. Lee; H.-M. So; E.-K. Jeon; H. Chang; K. Won; Y. H. Kim Analytical and Bioanalytical Chemistry 2008, 390, 1023.

[12] B. R. Baker; R. Y. Lai; M. S. Wood; E. H. Doctor; A. J. Heeger; K. W. Plaxco Journal of the American Chemical Society 2006, 128, 3138.

[13] O. Chen; R. Glaser; G. I. Likhtenshtein Biophysical Chemistry 2003, 103, 139. 


\section{List of Publications}

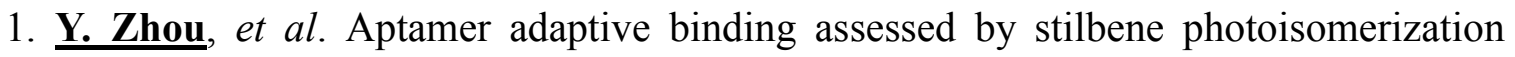
towards new aptasensors. In progress.

2. Y. Zhou, H. Chi, Y. Wu, R. S. Marks, T. W. J. Steele. Organic Additives Stabilize RNA Aptamer Binding of Malachite Green. Talanta, 2016, 160, 172-182.

3. Y. Zhou, H. Han, H. P. P. Naw, A. V. Lammy, C. H. Goh, S. Boujday, T. W. J. Steele. Real-Time Colorimetric Hydration Sensor for Sport Activities. Materials \& Design 2016, 90, 1181-1185.

4. J. Ping†, Y. Zhou †, Y. Wu, V. Papper, S. Boujday, R. S. Marks, T. W. J. Steele. Recent Advances in Aptasensors Based on Graphene and Graphene-Like Nanomaterials. Biosensors and Bioelectronics 2015, 64 (0), 373-385. (†equal contribution)

5. Y. Wu, V. Papper, O. Pokholenko, V. Kharlanov, Y. Zhou, T. W. J. Steele, R. S. Marks. New Photochrome Probe Allows Simultaneous pH and Microviscosity Sensing. Journal of Fluorescence 2015, 1-12.

6. V. Papper, O. Pokholenko, Y. Wu, Y. Zhou, P. Jianfeng, T. W. J. Steele, R. S. Marks. Novel Photochrome Aptamer Switch Assay (PHASA) for Adaptive Binding to Aptamers. Journal of Fluorescence 2014, 24 (6), 1581-1591. 